USE OF ADVANCED EMBEDDED PIEZOCERAMIC SENSORS FOR THE NON-DESTRUCTIVE EVALUATION OF REINFORCED CONCRETE

By

Dan Hughi, B. Eng, Toronto, 2010

A thesis presented to Ryerson University

in partial fulfillment of the

MASTERS OF APPLIED SCIENCE

In the program of

CIVIL ENGINEERING

Toronto, Ontario, Canada, 2013

(C) Copyright Dan Hughi, 2013 


\section{Author's Declaration:}

I hereby declare that I am the sole author of this thesis.

I authorize Ryerson University to lend this thesis or dissertation to other institutions or individuals for the purpose of scholarly research.

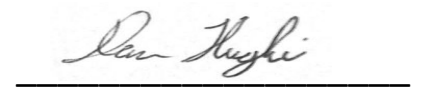

I further authorize Ryerson University to reproduce this thesis or dissertation by photocopying or by other means, in total or in part, at the request of other institutions or individuals for the purpose of scholarly research.

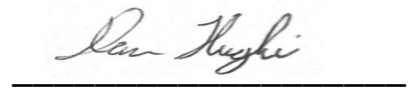




\title{
Abstract
}

\section{USE OF ADVANCED EMBEDDED PIEZOCERAMIC SENSORS FOR THE NON-DESTRUCTIVE EVALUATION OF REINFORCED CONCRETE}

\author{
By: Dan Hughi \\ Masters of Applied Science \\ Civil Engineering \\ Ryerson University, Toronto, Canada 2013
}

The present investigation deals with the development, and implementation, of embedded ultrasonic piezoceramic sensors as part of an active non-destructive health monitoring system for reinforced concrete members. As the world demand for economic, and sustainable energy rises, so does the demand for continuous structural health monitoring systems for high-importance structures such as, oil platforms and nuclear facilities. These facilities require the most reliable, and durable, monitoring systems in order to ensure their most economic, yet prudent operation. The proposed system was evaluated as a means for determining the development of concrete's early strength, as well as detecting first cracking in concrete. A series of small and full-scale concrete specimens were tested, and a relationship was drawn between the internal crack width of the members and the signal output of the proposed system. The MIRA 3D tomographer was also investigated for detecting the punching shear crack of thick concrete slabs. 


\section{Acknowledgements}

I wish to express sincere appreciation to my supervisor, Dr. Hesham Marzouk, for providing me with the opportunity to pursue my graduate work. The advice he provided and his never-ending patience were greatly appreciated throughout the completing of this work. Furthermore, I would like to thank Dr. Nabil Dawood for his support, and advice throughout the duration of this research.

I would like to extend my gratitude to Mr. Anto Sucic, and Mr. Kirllos Wahba, who as good friends, never hesitated to lend a helping hand and provide me with their best of advice. Lastly I would like to thank my parents for their continuous encouragement, support and affection, and my loving girlfriend who stood by me throughout this endeavor. 


\section{Table of Contents}

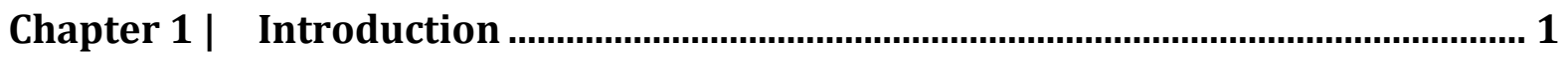

1.1 Objective and Scope

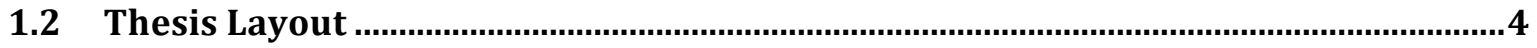

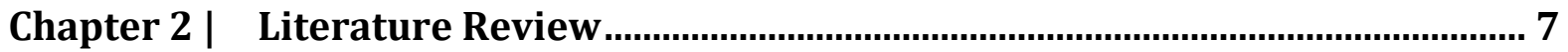

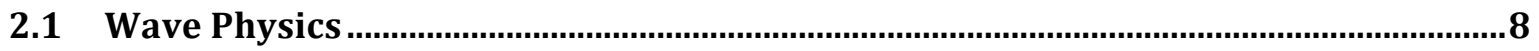

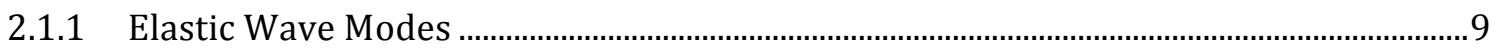

2.1.1.1 Rayleigh Wave .........................................................................................................................

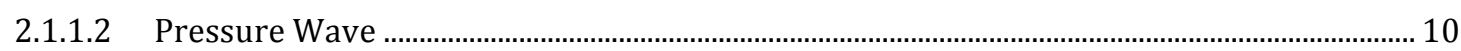

2.1.1.3 Shear Wave ..................................................................................................................................... 10

2.1.1.4 Guided Wave .............................................................................................................................. 11

2.1.2 Elastic Wave Behavior .................................................................................................. 12

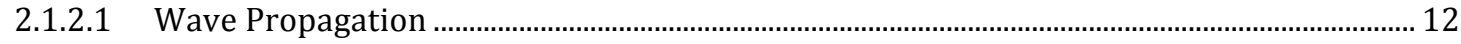

2.1.2.2 Wave Reflection and Refraction ................................................................................................. 13

2.1.2.3 Factors Affecting Wave Propagation in Concrete ...................................................................... 15

2.2 Applications of Embedded Piezoceramic Sensors in Concrete ................................ 16

2.2.1 Material Property Diagnostic............................................................................................... 16

2.2.2 Early-Age Performance Monitoring of Concrete .............................................................. 17

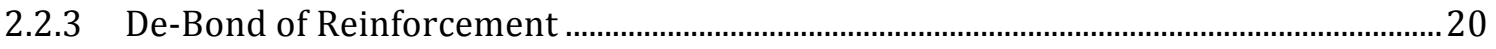

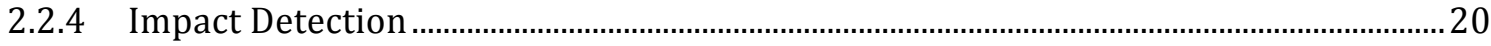

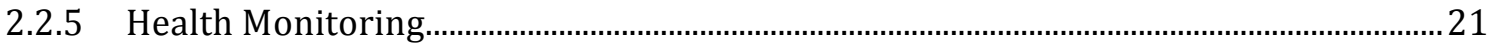

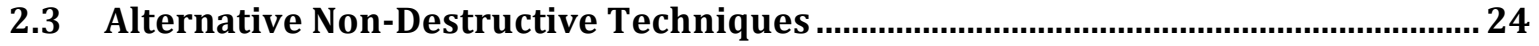

2.3.1 Health Monitoring Using MIRA 3D Tomographer ………….................................................24

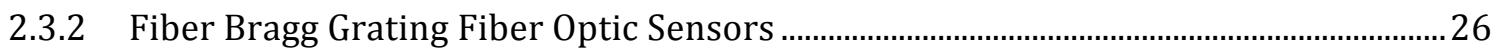

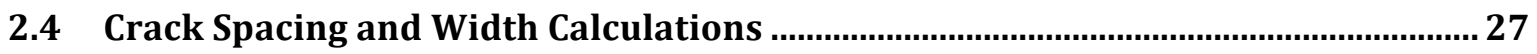

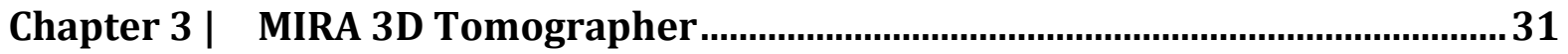

3.1 Experimental Program for Verifying the MIRA's Capabilities................................... 32

3.1.1 Depth of Signal Penetration and Effect of Reinforcement Ratio.......................................33

3.1.2 Reinforcement Location.................................................................................................. 34

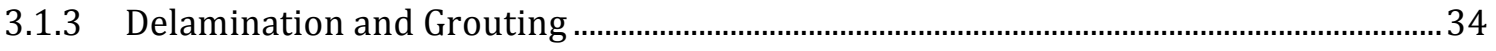


3.2 Experimental Results for Testing the MIRA's Capabilities .......................................... 34

3.2.1 Depth of Signal Penetration and Effect of Reinforcement Ratio ........................................34

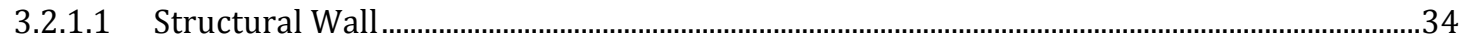

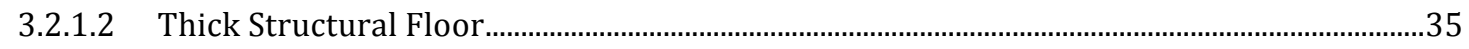

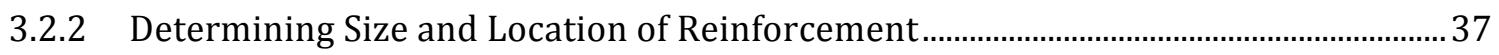

3.2.3 Delamination and Grouting Condition............................................................................... 38

3.3 Determining the Angle of the Punching Shear Crack for Deep Slabs ..................... 41

3.3.1 Experimental Program .................................................................................................... 41

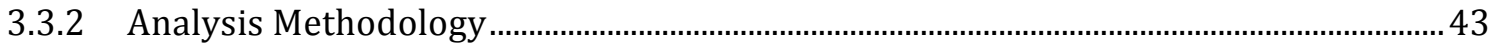

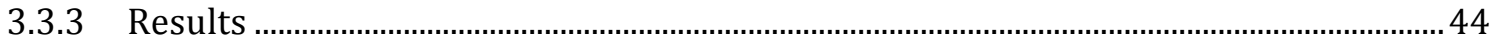

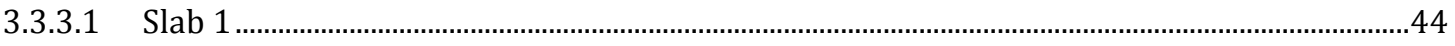

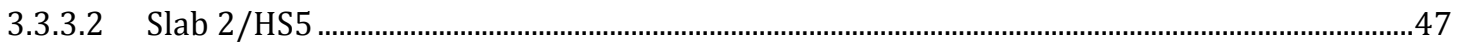

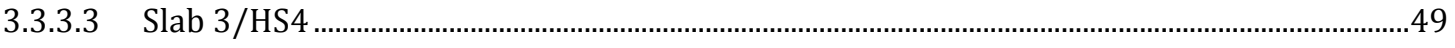

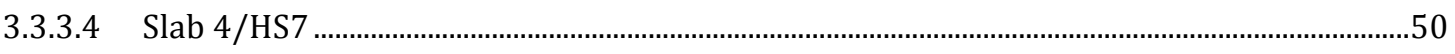

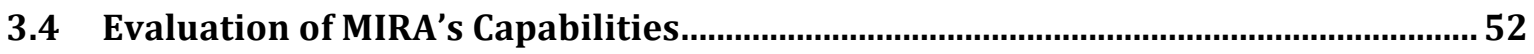

Chapter 4 | Proposed Piezoceramic System …........................................................... 55

4.1 Concept

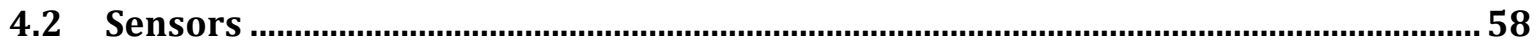

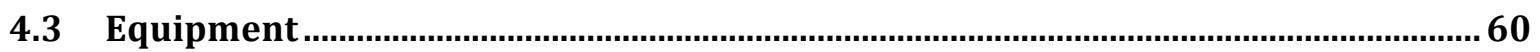

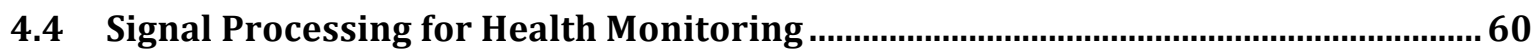

4.5 Signal Processing for Concrete Early Strength Prediction.....................................62

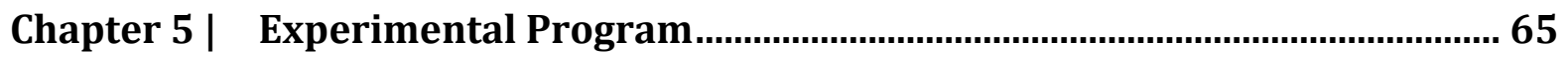

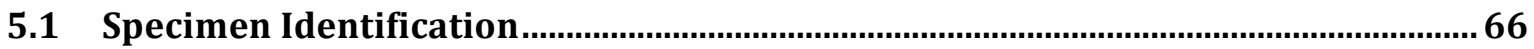

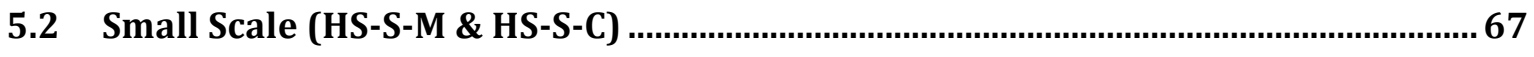

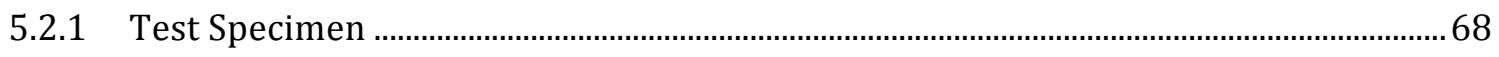

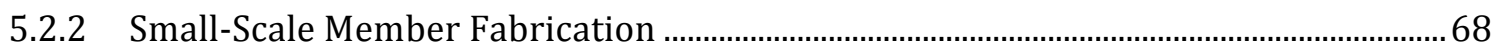

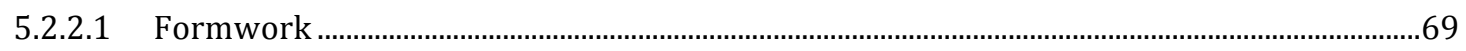

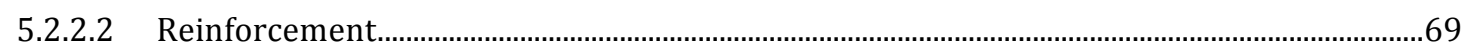

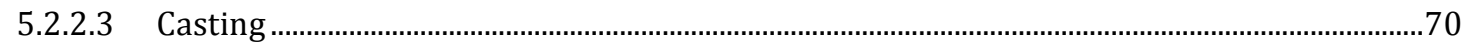

5.2.2.4 Curing

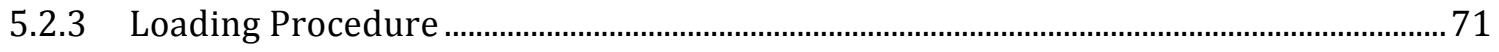

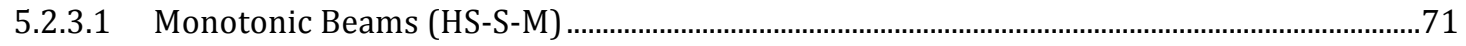




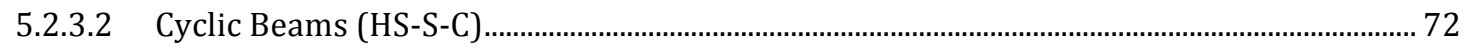

5.2.4 Measurement Devices.......................................................................................................... 72

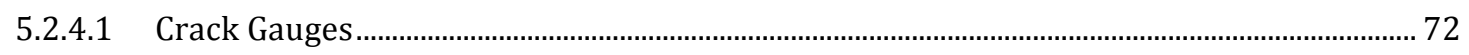

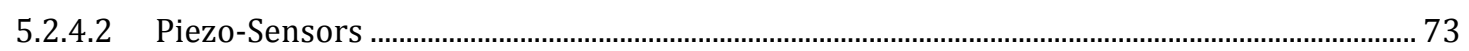

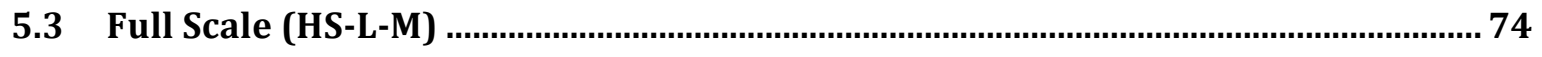

5.3.1 Beam Geometry and Loading Procedure.......................................................................... 74

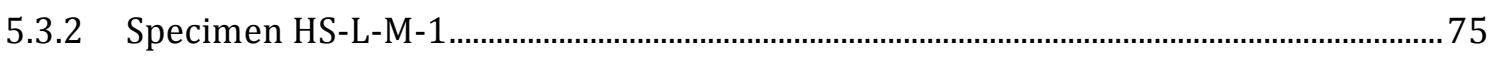

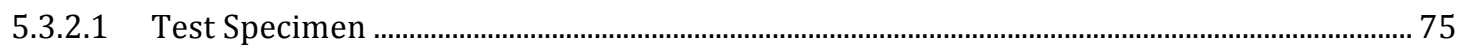

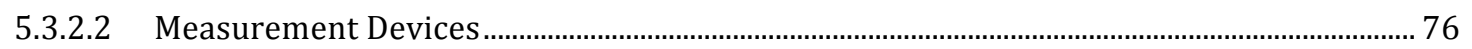

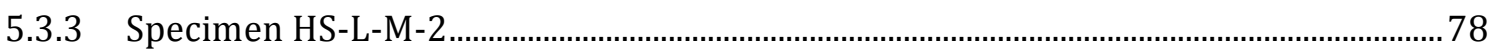

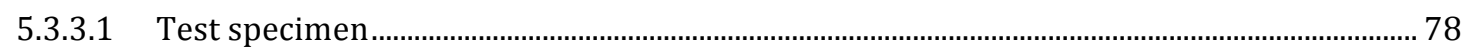

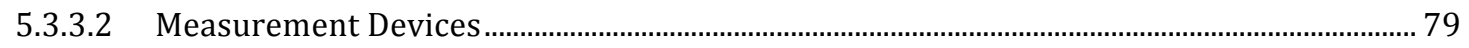

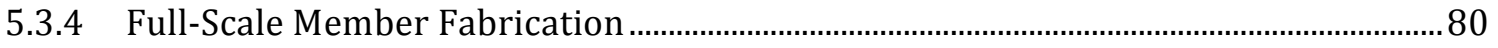

5.3.4.1 Formwork............................................................................................................................. 81

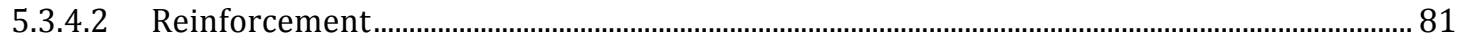

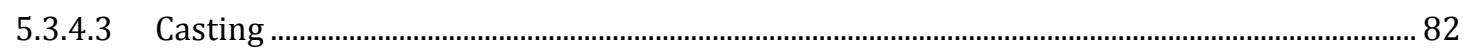

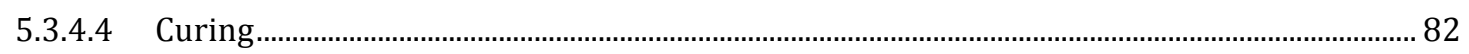

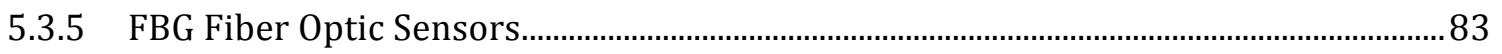

5.3.5.1 Arrangement of Fiber Optic Cable in Beam HS-L-M-1 ……........................................................ 84

5.3.5.2 Arrangement of Fiber Optic Cable in Beam HS-L-M-2 …………….......................................... 84

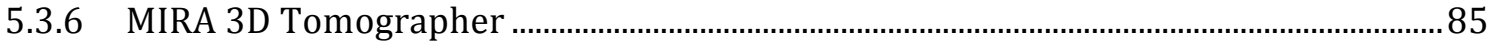

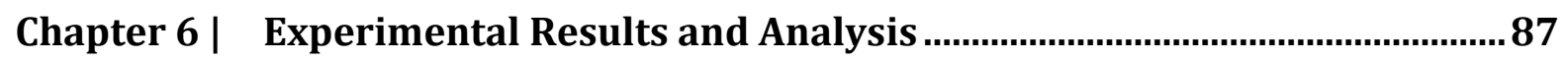

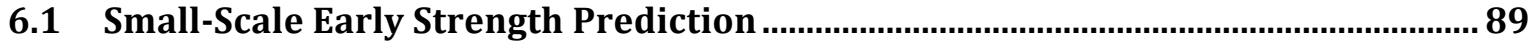

6.2 Small Scale Beams under Monotonic Loading (HS-S-M) ....................................... 95

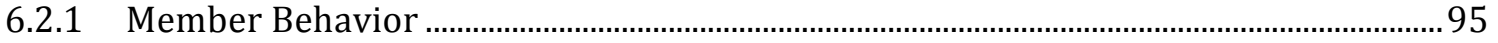

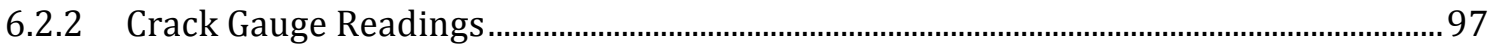

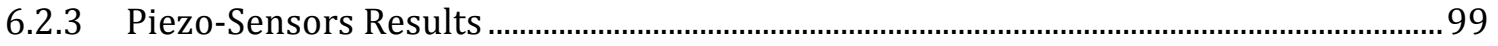

6.2.4 HMI-Strain Relationship Monotonic Beams ..................................................................... 102

6.3 Small Scale Beams under Cyclic Loading (HS-S-C) ................................................104

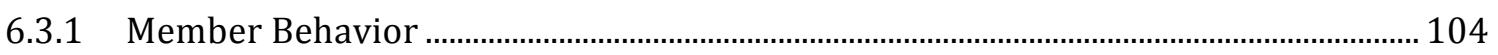

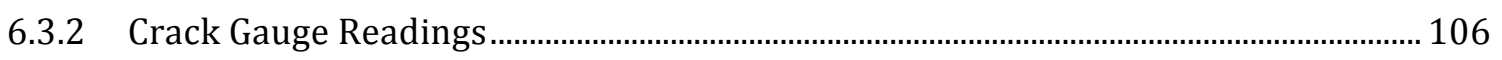

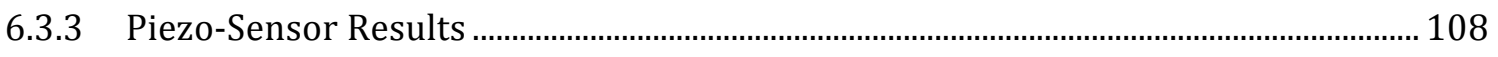

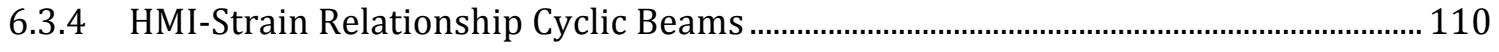


6.4.1 HMI-Crack Width Models

6.4.2 Verification of Crack Width-HMI Relationship Based on Results Obtained from the Monotonically Loaded Small Scale Beams

6.4.3 Verification of Crack Width-HMI Relationship Based on Results Obtained from the Cyclically Loaded Small Scale Beams.

6.4.4 Recommendation for a General Crack Width-HMI Equation........................................ 121

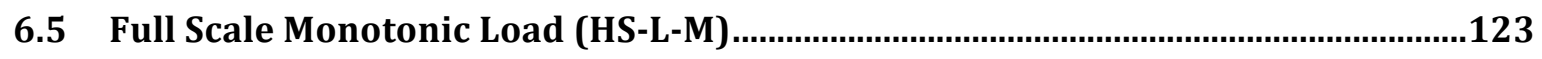

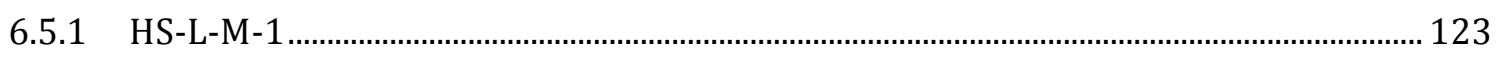

6.5.1.1 Member Behavior ..................................................................................................................124

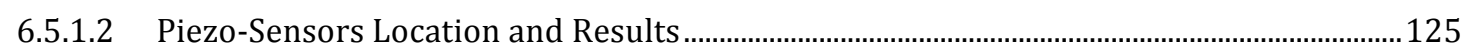

6.5.1.3 FBG Fiber Optic Sensors ...................................................................................................

6.5.1.4 Crack Width Measurements Using Piezo-System and Member Cracking Behavior .........131

6.5.1.5 Results Obtained Using the MIRA 3D Tomographer ………………………………………....136

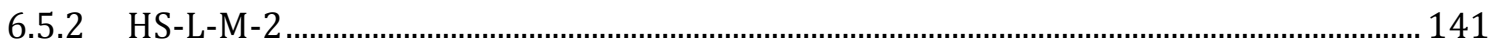

6.5.2.1 Member Behavior ………………………........................................................................ 141

6.5.2.2 Piezo-Sensors Location and Results ....................................................................................... 143

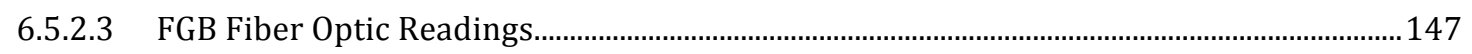

6.5.2.4 Strain Readings Using Proposed Model .......................................................................................... 149

6.5.2.5 Crack Width Measurements Using Piezo-System and Member Cracking Behavior ........152

6.5.2.6 Results Obtained With the MIRA 3D Tomographer................................................................159

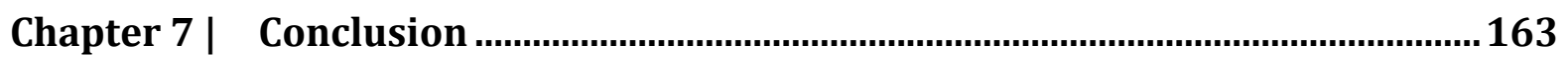

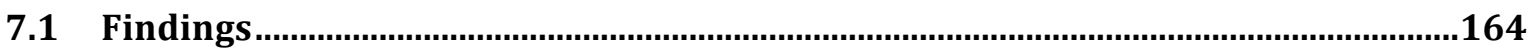

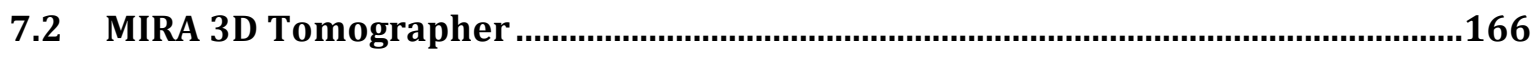

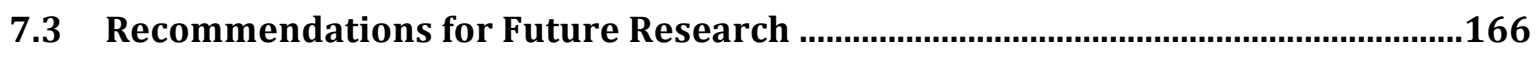

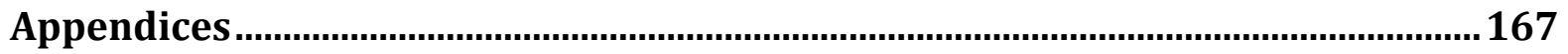

Appendix A | Compressive Strength Test Results Small Scale Beams ...........................169

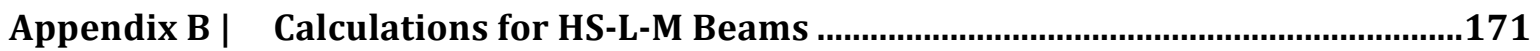

Appendix C | Crack Spacing and Crack Width Calculations …........................................175

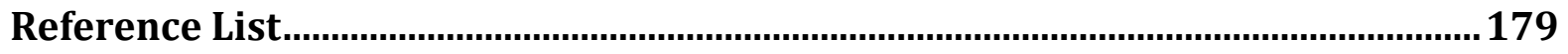




\section{List of Figures}

Figure 2.1: Releigh Wave Propagation in Determining Cracks (Aggelis \& Shiotani, 2007) ............................ 10

Figure 2.2: Wave Propagation in Solid Medium (Sansalone and Streett, 2007) ............................................. 11

Figure 2.3: Mechanism for Diffraction, Refraction and Reflection of Stress Waves ........................................ 14

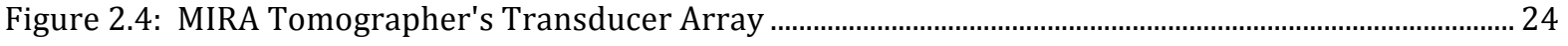

Figure 2.5: Signal Flow Using MIRA System when the Second Row of Transducers is Emitting Signal ....... 25

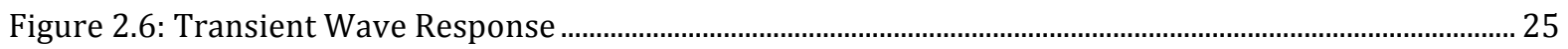

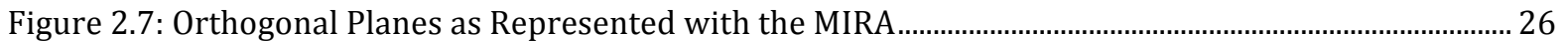

Figure 2.8: Transmission and Reflection Spectra of a Fibre Bragg Grating (Rizk el al. 2012) ...................... 27

Figure 2.9: Effective Embedment Thickness (CSA S474 2004 Code).................................................................. 28

Figure 2.10: Guidelines for Determination of Effective Concrete Area for Calculation of Crack Spacing

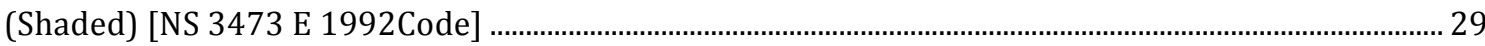

Figure 3.1: MIRA Test Specimens; (a) 2 m Deep Structural Wall; (b) $1.2 \mathrm{~m}$ Deep Structural Floor; (c) $0.4 \mathrm{~m}$

Deep Load Baring Wall; (d) 0.3 m Thick Exterior Basement Wall ............................................................... 33

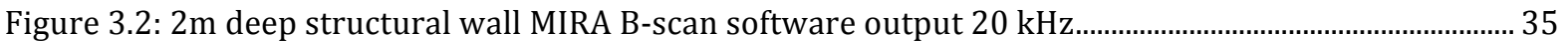

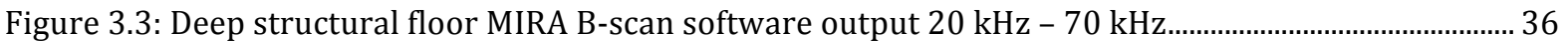

Figure 3.4: Basement Bearing Wall MIRA B-Scan software output 40kHz ...................................................... 37

Figure 3.5: Basement Exterior Wall Test Setup ................................................................................................. 38

Figure 3.6: Basement Exterior Wall MIRA Software Output 50 kHz................................................................ 39

Figure 3.7: Exterior Basement Wall MIRA Software Output 70 kHz.................................................................. 40

Figure 3.8: Test Surfaces of; (a) Slab 1; (b) Slab 2; (c) Slab 3; (d) Slab 4....................................................... 42

Figure 3.9: Slab 1 D-Scan Image; (a) 40 kHz; (b) 50 kHz; (c) 60 kHz; (d) 70 kHz ......................................... 46

Figure 3.10: Slab 2/HS5 D-Scan Image; (a) 30 kHz; (b) 40 kHz; (c) 50 kHz; (d) 60 kHz........................... 48

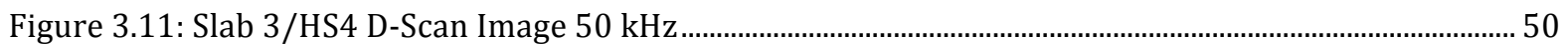

Figure 3.12: Slab 4/HS7 D-Scan Image; (a) 40 kHz; (b) 60 kHz ......................................................................... 51

Figure 4.1: Active Non-Destructive Health Monitoring Concept (Wu and Chang, 2006).............................. 56

Figure 4.2: Density of Transducer Mesh (Sun and Yan, 2008) ….................................................................... 57

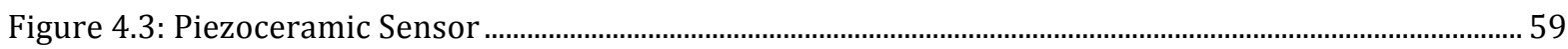

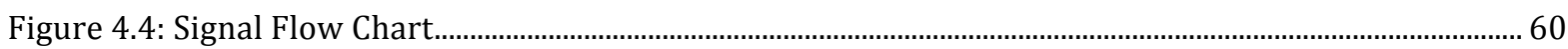

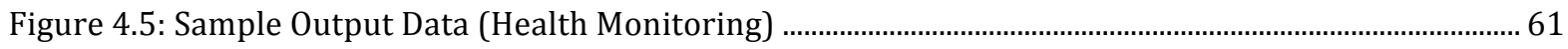

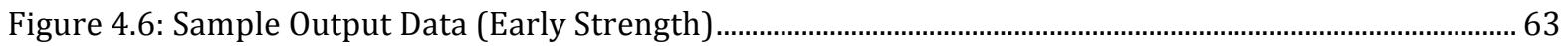

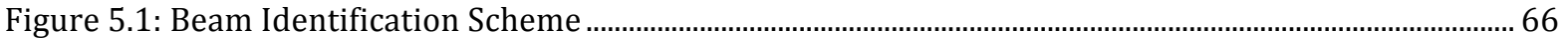

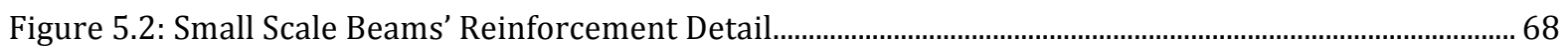

Figure 5.3: Formwork; (a) Assembly; (b) After Casting ..................................................................................... 69 
Figure 5.4: Placement of Steel Reinforcement Small-Scale Specimen, Top View .

Figure 5.5: Small Scale Beams after De-Molding

Figure 5.6: Loading Setup (a) Test Setup; (b) Three-Point Load Test Setup, Crack Gauge Location, and

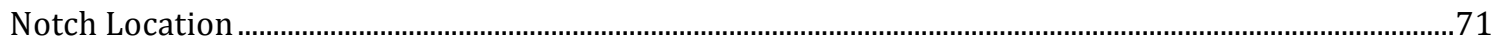

Figure 5.7: Loading Protocol Cyclic Beams (a) HS-S-C-1; (b) HS-S-C-2; (c) HS-S-C-3 ......................................72

Figure 5.8: Placement of Piezo-Sensors in Small Scale-Beams; (a) Piezo Sensors in Small-Scale Beams; (b)

Spatial Position of Piezo-Sensors in Small-Scale Beam.................................................................................. 73

Figure 5.9: Test Setup HS-L-M Beams (a) Loading Setup; (b) Detail of Loading Setup ..................................75

Figure 5.10: HS-L-M-1 Dimension and Reinforcement Detail..................................................................................76

Figure 5.11: Piezo-Sensor Placement (a) Spatial Position of Piezo-Sensor in Beam HS-L-M-1; (b) Signal

Paths for Flexural Control; (c) Signal Paths for Shear Control.....................................................................77

Figure 5.12: HS-L-M-2 Dimension and Reinforcement Detail..................................................................................79

Figure 5.13: Piezo-Sensor Placement (a) Spatial Position of Piezo-Sensor in Beam HS-L-M-2; (b) Signal

Paths for Flexural Control; (c) Signal Paths for Shear Control....................................................................8

Figure 5.14: Formwork; (a) Assembly; (b) Pre-Casting ...................................................................................

Figure 5.15: Steel Reinforcement Cage for Beam HS-L-M-2 Full-Scale Specimen; (a) Placed in Formwork;

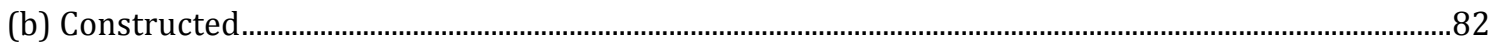

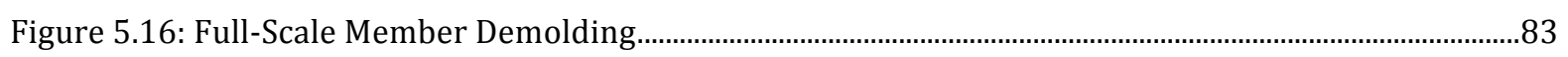

Figure 5.17: FBG Fiber Optic Sensors Spatial Position in the HSL Beam Series..................................................83

Figure 5.18: Arrangement of FBG Fiber Optic Sensors, and Piezo-Sensors in Beam HS-L-M-1 ......................84

Figure 5.19: Arrangement of FBG Fiber Optic Sensors, and Piezo-Sensors in Beam HS-L-M-2 ......................85

Figure 5.20: Vertical Scan Surface Using the MIRA 3D Tomographer.................................................................86

Figure 5.21: Horizontal Scan Surface Using the MIRA 3D Tomographer...........................................................86

Figure 6.1: Compressive Strength Based on Standard Concrete Cylinders .........................................................89

Figure 6.2: Sample Spectral Shift Beam from Frequency Domain of HS-S-M-2; (a) Day 1-4; (b) Day 5-7...90

Figure 6.3:Early Compressive Strength Predictions Using Piezoceramic Sensors ............................................94

Figure 6.4: Load-Deflection Graphs, Small Scale Monotonically Loaded Members.........................................96

Figure 6.5: Load-Strain Reading from Crack Gauge Equipment Small Scale Monotonically Loaded

Members; (a)HS-S-M-1; (b) HS-S-M-2; (c) HS-S-M-3; (d) HS-S-M-4 ........................................................97

Figure 6.6: Load-HMI Curves, Small Scale Monotonic Loaded Members; (a)HS-S-M-1; (b) HS-S-M-2;

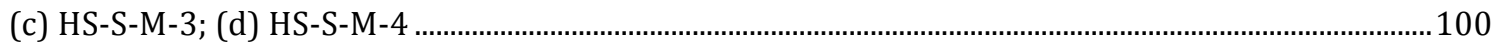

Figure 6.7: Summary Of Small-Scale Results; (a) Load-Strain Reading, Crack Gauges; (b) Load-HMI

Readings (c) Average HMI- Crack Width Relationship.............................................................................. 103

Figure 6.8: Load-Deflection Graph Small Scale Cyclically Loaded Members; (a) HS-S-C-1; (b) HS-S-C-2;

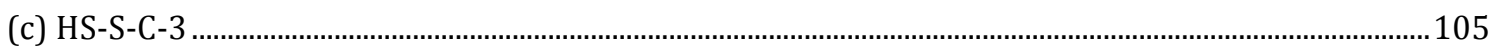

Figure 6.9: Load-Strain Reading from Crack Gauges, Small Scale Cyclically Loaded Members; (a) HS-S-C-1;

(b) HS-S-C-2; (c) HS-S-C-3 107 
Figure 6.10: Load-HMI Curves, Small Scale Cyclically Loaded Members; (a) HS-S-C-1; (b) HS-S-C-2; (c) HS-

S-C-3

Figure 6.11: HMI-Strain Relationship of Small Scale Cyclically Loaded Beams; (a) HS-S-C-1; (b) HS-S-C-2;

(c) HS-S-C-3

111

Figure 6.12: Average HMI-Crack Width Relationship as Suggested by Small Scale Cyclically Loaded Beams

Figure 6.13: Comparison of HS-S-M and HS-S-C HMI-Crack Width Models, and Direct Crack Width

Readings Using Crack Gauges, Using Experimental data from HS-S-M Beams; (a) Beam HS-S-M-1; (b) HS-S-M-2; (c) HS-S-M-3; (d) HS-S-M-4; (e) Computed Standard Deviation of Cyclic and Monotonic Models

Figure 6.14: Comparison of HS-S-M and HS-S-C HMI-Crack Width Models, and Direct Crack Width

Readings Using Crack Gauges, Using Experimental data from HS-S-C Beams; a) Beam HS-S-C-1; b)

HS-S-C-2; c)HS-S-C-3; d) Computed Standard Deviation of Cyclic and Monotonic Models 120

Figure 6.15: Universal HMI-Crack Width Relationship for Piezo-System. 122

Figure 6.16: Load-Defection Curve for Beam HS-L-M-1

Figure 6.17: Piezo-Sensor Spatial Position 126

Figure 6.18: Load-HMI Graphs for HS-L-M-1; (a) Support Location; (b) Quarter-Span; (c) Shear Crack;

(d) Compression Block

Figure 6.19: FBG Fiber Optic Readings from Beam HS-L-M-1; (a) Location of Working Sensors; (b)

Readings Obtained from FBG Sensor 130

Figure 6.20: Shear Crack Behavior for Beam HS-L-M-1; (a) Crack Width Measurements; (b) Cracking

Behavior Left Side; (c) Cracking Behavior Right Side 132

Figure 6.21: Effective Shear Depth Location Behavior for Beam HS-L-M-1; (a) Crack Width Measurements;

(b) Cracking Behavior Left Side; (c) Cracking Behavior Right Side 134

Figure 6.22: Support Location Behavior for Beam HS-L-M-1; (a) Crack Width Measurements; (b) Cracking Behavior Left Side; (c) Cracking Behavior Right Side 135

Figure 6.23: Vertical Scan Direction Beam HS-L-M-1 Healthy State; (a) Theoretical Location of Steel

Reinforcement; (b) MIRA Software Output

Figure 6.24: Vertical Scan Direction Beam HS-L-M-1 Damaged State; (a) Observed Cracking Pattern on Scanned Surface; (b) MIRA Software Output 138

Figure 6.25: Horizontal Scan Direction Beam HS-L-M-1 Healthy State; (a) Theoretical Location of Steel

Reinforcement; (b) MIRA Software Output 139

Figure 6.26: Horizontal Scan Direction Beam HS-L-M-1 Damaged State; (a) Observed Cracking Pattern on

Scanned Surface; (b) MIRA Software Output..................................................................................................140

Figure 6.27: Load Defection Curve Beam HS-L-M-2 ….................................................................................142

Figure 6.28: Piezo-Sensors Spatial Position in Beam HS-L-M-2 143 
Figure 6.29: Flexural Control Load-HMI Graph for HS-L-M-2; (a) Support Location; (b) Distance dv from

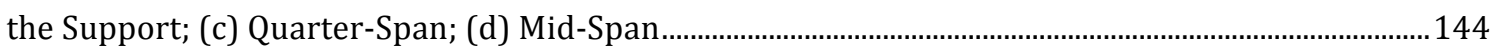

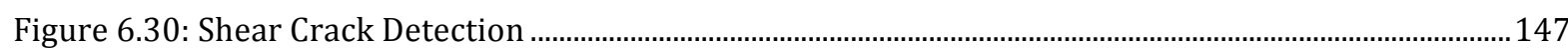

Figure 6.31: FBG fiber optic readings from beam HS-L-M-2; (a) Location of working sensors; (b) Readings obtained from FBG Sensors 148

Figure 6.32: Strain Reading Obtained from Beam HS-L-M-1; (a) Adjacent to Support Location; (b) Quarter Span Location; (c) Adjacent to Mid-Span Location; (d) Mid-Span Location ...............................................150

Figure 6.33: Results obtained at the Mid-Span of Beam HS-L-M-2 (a) Load-Crack Width Graph yielded by Piezo-Sensors and FBG Cable; (b) Observed cracking pattern, and location of FBG sensor. 153

Figure 6.34: Results obtained adjacent to the support region HS-L-M-2; (a)Load-Crack Width Graph as yielded by piezo-sensors; (b) Observed cracking pattern between sensors Pair 9-3, left side of the beam; (c) observed cracking pattern between sensor pair 10-6, right side of the beam 154

Figure 6.35: Results obtained at $d_{v}$ from the support of beam HS-L-M-2; (a) Load-Crack Width Graph yielded by Piezo-Sensors and FBG Cable; (b) Observed cracking pattern between sensors Pair 2-3, left side of the beam; (c) Observed cracking pattern between sensor Pair 6-7, right side of the beam

Figure 6.36: Shear Crack width measurements for beam HS-L-M-2; (a) Load-Crack Width Graph yielded by Piezo-Sensors and FBG Cable; (b) Observe cracking pattern between sensors Pair 9-3, left side of the beam; (c) Observed Cracking Pattern Between Sensor Pair 10-6, right side of the beam.............157

Figure 6.37: Horizontal Scan Direction Beam HS-L-M-2 Healthy State; (a) Theoretical Location of Steel

Reinforcement; (b) MIRA Software Output. 159

Figure 6.38: Horizontal Scan Direction Beam HS-L-M-2 Damaged State; (a) Observed Cracking Pattern on Scanned Surface; (b) MIRA Software Output. 160 


\section{Notations}

A : Amplitude of Stress Wave

$A_{f n, 0}$ : Amplitude of Received Wave at Specific Frequency $f$ at the Healthy State

$A_{f n, t}$ : Amplitude of Received Wave At Specific Frequency $f$ at a Particular Time " $\mathrm{t}$ "

$A_{i}:$ Amplitude of an Induced Wave

A $_{\text {piezo : }}$ Area of Piezoceramic Patch

A Reflected : Amplitude of a Reflected Wave

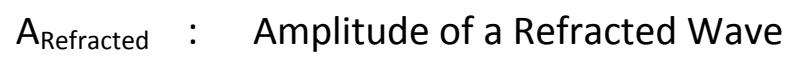

$A_{s}:$ Area of Steel Reinforcement; in the Effective Thickness

$a_{1}: \quad$ Distance to the Top of Outer Layer of Steel Reinforcement

$a_{2}$ : Distance to the Top of Inner Layer of Steel Reinforcement

b : Base Width of a Section

$\beta$ : Factor Taking Loading Procedure into Account

C : Stress Wave Velocity, Concrete Cover

$C_{b}:$ Velocity of One-Dimensional Stress Wave

c.c. : Concrete Cover

$C_{p}:$ Pressure Wave Velocity

$C_{r}:$ Reyleigh's Wave Velocity

$C_{s}:$ Shear Wave Velocity

c.w. : Crack Width

d : Distance

$\mathrm{d}_{\text {be }}^{\prime}$ : Effective Bar Diameter

E : Modulus of Elasticity

$E_{h j}: \quad$ The Healthy State Energy of a Wave Oscillating at the $j^{\text {th }}$ Frequency

$E_{i j}:$ The Instantaneous Energy of a Wave Oscillating at the $j^{\text {th }}$ Frequency

$\varepsilon$ : Strain

$\varepsilon_{1}$ : Smallest Tensile Strain in the Effective Embedded Zone; Principal Strain of Tensile Reinforcement

$\varepsilon_{2}: \quad$ Largest Tensile Strain in the Effective Embedded Zone 


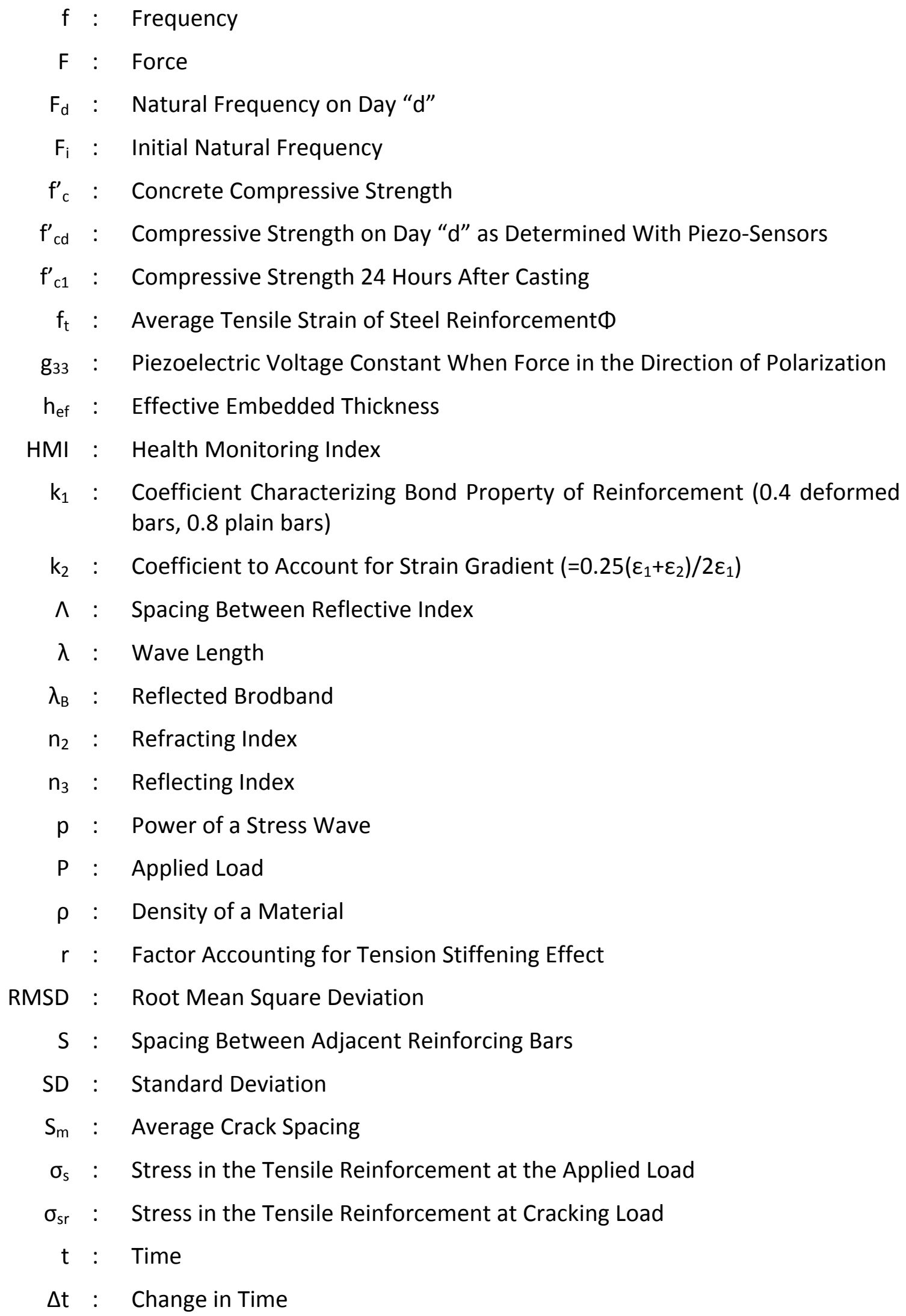




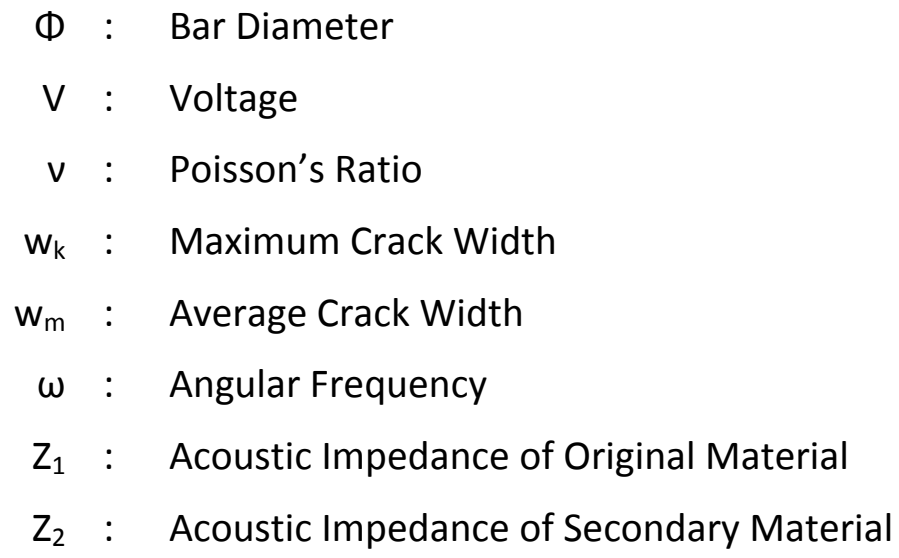





\section{Chapter $11 \quad$ Introduction}

The following investigation examines the development, and implementation, of piezoceramic sensors as part of an active non-destructive health monitoring system for reinforced concrete members. As the world demand for economic and sustainable energy rises, the demand for nuclear power containment and offshore platforms development has increased dramatically. These indispensable structures require the most reliable, and durable, monitoring systems in order to ensure their most economic, yet prudent operation. The proposed system uses permanently embedded sensors to actively assess the structural condition of these high-risk facilities throughout their lifetimes. In the study a series of small and full-scale concrete specimens were tested. The results of these tests are presented, as to demonstrate validity of the proposed system, and provide a means of establishing an active non-destructive system that not only assesses the extent of damage, but also specifies its location within the reinforced concrete member. 
Over the last few years, a large number of offshore platforms, and nuclear power containment structures were developed in order to respond to the increasing worldwide demand of economic and sustainable energy. These mega important structures, setting the standards for safety and extended lifespans (exceeding 100 years), requiring the highest level of monitoring system based on reliable and durable sensors. The current study focuses on the development of an effective, yet inexpensive active health monitoring system that will eliminate the pitfalls of traditional non-destructive techniques. The proposed system uses embedded piezoceramic sensors, in order to provide real time assessments of concrete members, and establish a means for preventive health monitoring. This study also investigates the implementation of these piezo-sensors as a permanent, continuous recording instrument that monitor the propagation of cracks within concrete members.

The occurrence of cracking is part of the structural condition of reinforced concrete member. The low tensile strength of concrete requires that steel reinforcement be introduced to take the tensile strains experienced by the material. The steel is mobilized when the concrete exceeds its tensile capacity and cracking occurs. Structures exposed to harsh environments usually have much stricter requirements for the amount of cracking that is permitted to occur before the building is considered unsafe, for example offshore structures. The harsh marine environment can prove to be fatal to the longevity of a reinforced concrete building. A crack in the protective concrete casing housing the reinforcement, would allow the infiltration of water in the concrete matrix, oxidizing the reinforcement within. The oxidation of the reinforcement would prove rather fatal to the structural integrity of any structure, and thus is highly controlled by the stringent design requirement provided by the various international building codes. Cracking in reinforced concrete structures are not highly controlled in terms of deterioration of structures, the intended utilization of the building is also very important. Structures such as water tanks, silos, containment structures, and nuclear reactor facilities, have zero tolerance on cracking. The appearance of cracking in such a facility would render it un-serviceable, and cause devastating environmental problems. The development of a system to detect the presence of cracking in these mega important structures would ensure the safe and prudent 
operation desired of any facility. The proposed piezoceramic system in this investigation is developed to eliminate the pitfalls of conventional non-destructive techniques, and facilitate the evaluation of these imperative civil structures.

\subsection{Objective and Scope}

The current investigation aims at the development of an embedded active non-destructive health monitoring system for the evaluation of reinforced concrete members. The system must be developed to be simple, reliable, durable, and energy efficient, as to address some of the deficiencies of the available alternative test methods. It also must provide a means to locate and quantify the appearance of internal cracking, and provide warnings of a possible member failure. The embedded system is required to provide real time assessments of the member/building's condition, and it must be self-sustaining, minimizing the need for an operator. The function of the active health monitoring system is to continuously assess various components of a reinforced concrete structure, and keep data logs of the building's performance over time. The information provided by these data logs can be assessed by maintenance personal at any given time during the building's life-span to prioritize maintenance scheduling for building rehabilitations. The intended application of this system, although not limited, is for buildings of high-importance, where a member failure is intolerable and could cause catastrophic disasters (ie. Nuclear containment structures, offshore oil platforms, etc.). The following five objectives were the focus of the investigation:

- Determining the influence that induced damage, and curing of concrete, have on the vibration characteristic of a set concrete section

- Establishing a signal processing tool to quantify the effects that curing and damage have on the induced signal

- Relating the signal processing value to an equivalent concrete strain as based on conventional measuring techniques 
- Verify the developed relationship between the signal processing value and the concrete strain using full scale experimental samples to illustrate a real world application of the system

- Compare the obtained results from the piezo-system to the various other nondestructive testing methods available

To achieve the desired objectives, proposed system is developed based on numerous testing strategies. A series of small-scale experimental tests were conducted to determine the applicability of piezoceramics to assess the condition of reinforced concrete beams. The results of the small-scale experimental programs were later used to develop a means of quantifying the detected damage of two full-scale test samples. The quantification of the detected damage was developed in terms of crack width measurements, and compared to other well-established sensing systems available on the market today.

\subsection{Thesis Layout}

The chapter following this introduction will present the research, development, and evaluation that were involved in the development of the piezoceramic system. A review of the literature utilized in the development of the entire investigation is presented in Chapter 2. This chapter will present the physics principals that govern the propagation of ultrasonic stress waves within the confines of a finite element, followed by the various applications of piezoceramics in the ultrasonic testing of concrete members. Also the alternative evaluation techniques that were used in the completion of this investigation are discussed in Chapter 2. Ending with a review of the various equations provided by the Canadian and Norwegian building codes for approximating a beams cracking behavior

Chapter 3 presents some of the previous work done using the MIRA 3D tomographer. Here, the first section illustrates the capabilities of the MIRA, testing the various specifications of the equipment, as to better understand its working principal. The section then follows with an investigation that was carried out to determine the angle of punching shear cracks in 
thick concrete slabs. The section details the experimental samples tested in the investigation, and the finding obtained from the equipment.

Chapter 4 presents the theory, and components that comprise the piezo-ceramic system. The concept of active health monitoring is presented, as to clarify the scope of the research. The section will then present the sensors that are embedded into the concrete matrix as part of the piezo-system, along with the equipment used to power them. The chapter will also provide a brief description of the signal processing used during the testing process.

Chapter 5 will present the undertaken experimental program. The detailing, construction and testing of the various experimental samples are defined in this chapter. This chapter begins by describing the small-scale experimental program, as well as the data recording equipment, and their installations. This chapter follows with the detailing, construction, and testing procedures for the full-scale members, along with the descriptions of the alternative testing equipment utilized in the completion of the experimental program.

Chapter 6 begins by presenting the results and analysis of the experimental work. The chapter begins by discussing the findings of the small-scale experimental beams. The structural responses of the members are first presented, followed by the readings obtained from the test equipment ending with a brief analysis of the results obtained from each loading class. The collected results of the two loading types (monotonic and cyclic) of the small-scale members are then combined, as to provide the desired means of interpreting the sensor signals from the piezo-ceramics into the desired crack width measurements. The chapter then discusses the results obtained from the full-scale members. The data is presented in the same order as the small-scale experimental results, followed by the application of the developed piezo-sensor signal-crack width relationship to the results obtained by the full-scale experimental program.

Finally, Chapter 7 presents the findings and future recommendation for this research. 



\section{Chapter 2 Literature Review}

Lifetime nondestructive monitoring of reinforced concrete structures is highly important. As concrete is an extremely complex material, and as the current infrastructure continues to age, it is crucial for engineers to have a quick, simple, and effective way of determining the structural integrity of these structures. Over the past years, development of ultrasonic stress wave testing has helped to improve the monitoring of these crucial structures, as a form of in-situ nondestructive testing.

The advancements in understanding of stress wave propagation in concrete have spawned a new era of ultrasonic testing. As proposed by Wang et. al (2000), the concept of active lifetime health monitoring of structures is a very desirable field. This concept utilizes embedded piezoceramic patches in order to provide maintenance strategy with real time 
information of the structures integrity. The application would eliminate the current requirement for bulky equipment, and on site testing. Piezoceramic patches have the advantage of low cost, and quick response in large structures where conventional means of testing are tedious and ineffective. Sensors can be installed in a predetermined array, and can monitor a vast number of structural characteristics of concrete. The sensors have been successfully applied in determining the Poisson's ratio, modulus of elasticity, and curing condition; as well as determining the delamination of reinforcement, and cracking within the concrete matrix.

The scattering and attenuation of ultrasonic stress waves within the concrete allow engineers to determine a large amount of information about the interior quality of reinforced concrete members. The information that can be obtained includes, but is not limited to: the mix's gradation, de-bonding of reinforcement from concrete, ensuring the proper placement of reinforcement, locating cracks within structural elements, and the overall internal structure of the concrete matrix. Ultrasonic test methods have been proven effective at acquiring such information quickly, and provide engineers with effective methods of determining the internal defects found within reinforced concrete.

The following section presents the literature used to develop, and complete the research objective. Literary research was also presented for the apparatuses used in the completion of the research work such as the MIRA 3D tomographer and FBG fiber optic sensors. The previsions of the Canadian and Norwegian building codes were applied in the research and are presented herein.

\subsection{Wave Physics}

The following section presents a brief introduction of the various modes, and equations, governing the physical behavior of ultrasonic wave propagation within solid mediums. Ultrasonic frequencies, although subjective, are commonly considered to be any acoustic emission surpassing a frequency of $20,000 \mathrm{~Hz}$. Although a signal frequency could be adjusted within any medium, the speed of each wave mode is governed by the material's 
properties. Applying an extensive understanding of the behavior of ultrasonic waves has helped researchers to develop the large variety of new applications for ultrasonic technologies.

\subsubsection{Elastic Wave Modes}

When an instantaneous stress is implemented into an object, such as the impact from a hammer, four fundamental stress waves are generated. For concrete, the primary wave propagation modes considered for stress wave analysis are: the Rayleigh wave (R-Wave), the pressure wave (P-Wave), and the shear wave (S-Wave). Although very different in their nature, the three waveforms are all created simultaneously from the induction of an instantaneous stress. The fourth waveform that is generated is called a Lamb wave. Although very useful in non-destructive testing of thin steel plates, this wave mode is not commonly applied for non-destructive testing of concrete samples.

To better understand the information carried by each wave mode, and the expected behavior of the waves within the concrete member; the propagation behavior and nature of each waveform has to be studies. Understanding each mode shape's behavior, facilitates the analytical aspect of the non-destructive testing

\subsubsection{Rayleigh Wave}

The Rayleigh wave is a surface wave that travel in planes normal to the testing surface thus the particles are vibrated perpendicular to the direction of travel. The depth of penetration of Reylaigh waves is directly proportional to the wavelength. This wave mode is very useful for determining the appearance of surface cracking in concrete specimens. Cracks that propagated deeper into the test specimen then the wavelength of the transmitted wave will completely attenuate this wave mode. Thus a receiver placed on the opposite side of the fault from where the stress wave is induced would not sense the disturbance (Aggelis \& Shiotani, 2007). Figure 2.1 demonstrated how the Rayleigh wave having a wavelength smaller than the crack depth, would be shadowed by the receiving transducer; whereas if 
the wavelength is greater than the depth of the crack the signal will be received by a secondary transducer. This enables to estimate the penetration depth of a surface cracks within a concrete member. Rayleigh waves can be estimated to have a velocity of $55-60 \%$ of that of a P-Wave, and are directly proportional to the velocity of the shear wave.
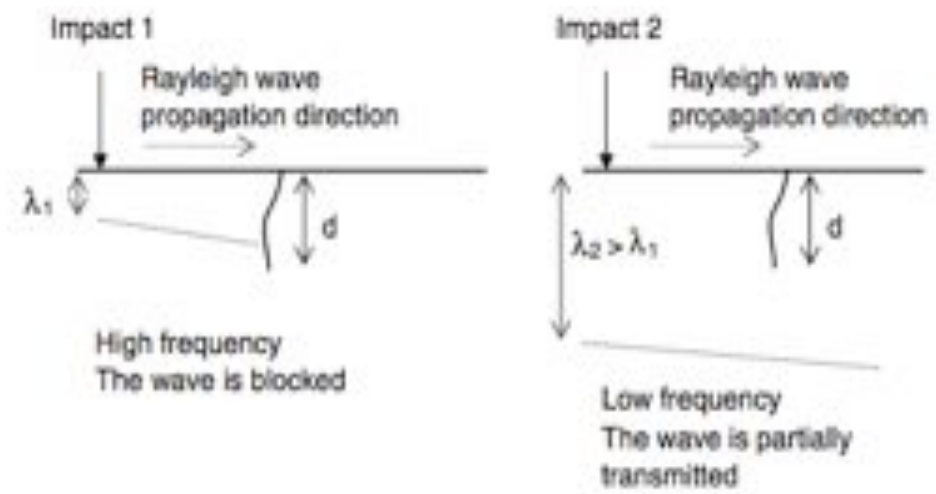

Figure 2.1: Releigh Wave Propagation in Determining Cracks (Aggelis \& Shiotani, 2007)

\subsubsection{Pressure Wave}

A pressure wave (longitudinal wave) is a linearly elastic wave traveling within the bulk of the solid specimen. The polarization of P-Waves is longitudinal and vibrates particles in the direction of travel. This type of wave is very common in non-destructive techniques such as the impact-echo. The application is useful for one-dimensional interpretation of the internal structure. In the case of impact-echo, a hammer is used to introduce the stress wave into a medium, from where a one-dimensional transducer receives the P-Wave giving uniaxial information about the specimen's internal structure. The wave speed in concrete is typically around $4000 \mathrm{~m} / \mathrm{s}$.

\subsubsection{Shear Wave}

The shear wave (transverse wave) is a linearly elastic wave traveling within the bulk of the material sample. Unlike the P-Wave, the shear wave's motion is perpendicular to the direction of travel, and provides a much clearer image of the internal structure of the concrete specimen. By oscillating particles perpendicular to the direction of travel, this 
wave mode excites particles in shear. This oscillation provides a much higher chance that the induced stress wave will come in contact with an imperfection within the medium. Since the range of sample that the wave travels through is much larger than the P-Wave, smaller imperfections can be detected and documented. Figure 2.2 differentiates between the motions of the three waves modes within a solid specimen. The MIRA 3D tomographer is a non-destructive testing system that utilizes shear wave reflections to examine the internal condition of reinforced concrete members. The shear wave travels at approximately $60 \%$ the velocity of the P-Wave.

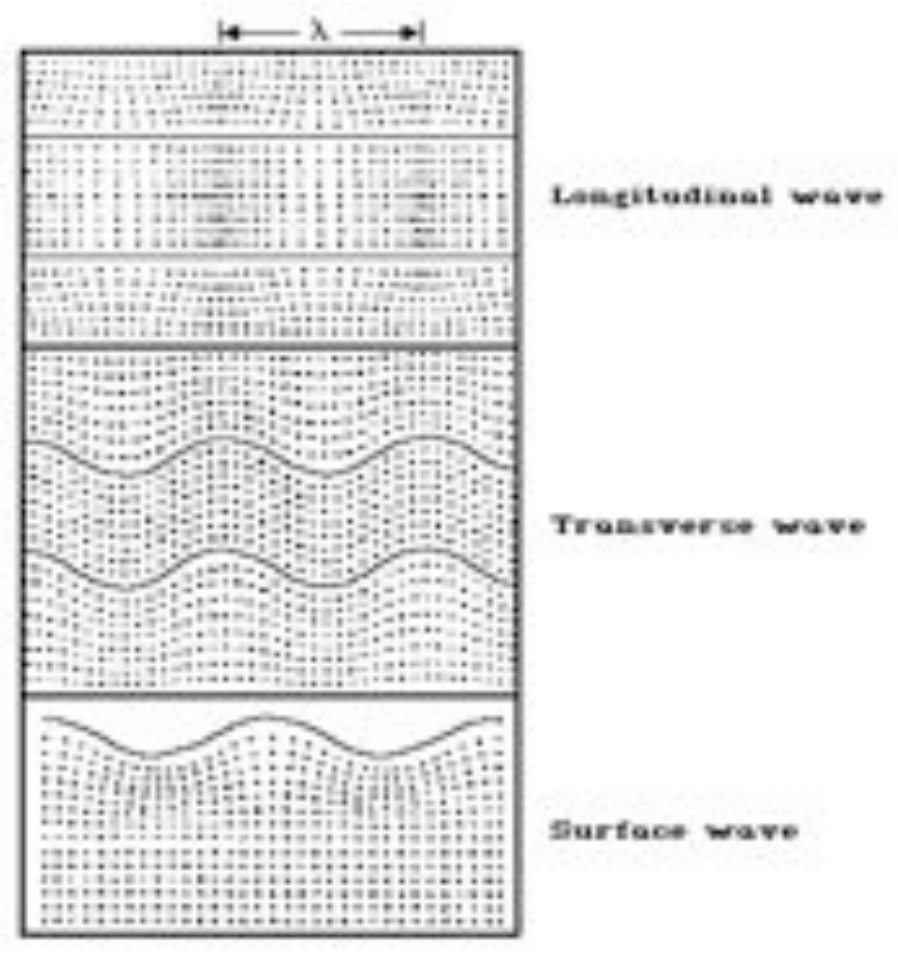

Figure 2.2: Wave Propagation in Solid Medium (Sansalone \& Streett, 2007)

\subsubsection{Guided Wave}

The principal of guided waves is most commonly used in the non-destructive testing of steel piping, and automotive components. The guided wave is generated using a high-frequency transducer that emits a sweep-frequency harmonic excitation into the test medium. Utilizing a sweep frequency helps examine the true vibration characteristic of a medium, or ultrasonic signature. This sweep frequency ensures that the induced stress wave is tuned to 
the resonant frequencies of the material, and thus facilitates its transmission. Unlike the longitudinal and transverse waves, the guide wave is not a pulse-generated wave; rather it is generated by a continuous sinusoidal stimulation. The continuous stimulation, along with the implementation of a sweep frequency ensure that the stress wave can be transmitted over a much greater distance than pulse based non-destructive testing systems.

\subsubsection{Elastic Wave Behavior}

\subsubsection{Wave Propagation}

The principals of wave propagation are very useful for analysis of ultrasonic signals. Piezoceramic patches can be used to induce stress waves into the test specimen. The stress waves are then analyzed based on frequency, amplitude, and velocity as to understand the internal condition of the material. These stress waves must conform to the fundamental equations of stress, and electromagnetic wave propagation. The speed of any wave is given as a function of the wave's frequency, and wavelength. Equation 2.1 shows that the speed of the wave (C), is equal to the wavelength $(\lambda)$, multiplied by the frequency of the wave (f).

$$
C=\lambda \cdot f
$$

The material it travels through governs a stress wave's velocity. The velocity of the shear wave can be determined using Equation 2.2 (Sansalone and Streett, 2007). The velocity is proportional to the modulus of elasticity, Poisson's ratio, and density of the material. This means that the shear wave velocity between two materials is always going to differ. $E$ is the modulus of elasticity, $\rho$ is the density, and $v$ is the Poisson's ratio of the material.

$$
C_{s}=\sqrt{\frac{E}{2(1+v) \rho}}
$$


Similarly the pressure wave velocity is calculated based on Equation 2.3 (Sansalone and Streett, 2007).

$$
C_{p}=\sqrt{\frac{E(1-v)}{(1+v)(1-2 v) \rho}}
$$

The velocity of the Reyleigh wave, as previously mentioned is a function of the shear wave velocity and is computed by using Equation 2.4 (Sansalone and Streett, 2007).

$$
C_{r}=\frac{0.87+1.12 v}{1+v} C_{s}
$$

If the velocities of the shear and pressure wave are determined for any given material, then the modulus of elasticity, and Poisson's ratio in the region are known. The ability to determine these two material properties allow engineers to quickly and effectively determine the curing condition of a concrete structure, without the need for coring. The analysis provides a quick and effective system for monitoring these material properties throughout the lifetime of the structure and provides a general representation of the approximate concrete strength at any given time throughout the structures useful service life. The study carried out by Li et. al (2009) was focused on this particular application, and will be discussed in more detail.

\subsubsection{Wave Reflection and Refraction}

The interaction between the various materials in the reinforced concrete member is important characteristic for the analysis of ultrasonic guided waves. As the acoustic impedance between various mediums varies, so does the propagation of stress waves. The 
most important of which is the interaction of a stress wave with a concrete/air interface, or crack location. When a stress wave comes in contact with this interface, the wave is split into two components, a through component, or refracted wave, and a reflected component, as illustrated in Figure 2.3. A third stress wave is created at the edge of the crack opening caused by the diffraction of the incident wave. This diffracted wave travels spherically through the medium centered at the edge of the crack. The amplitudes of these three wave components are fractions of the induced stress wave's amplitude upon incident with the interference.

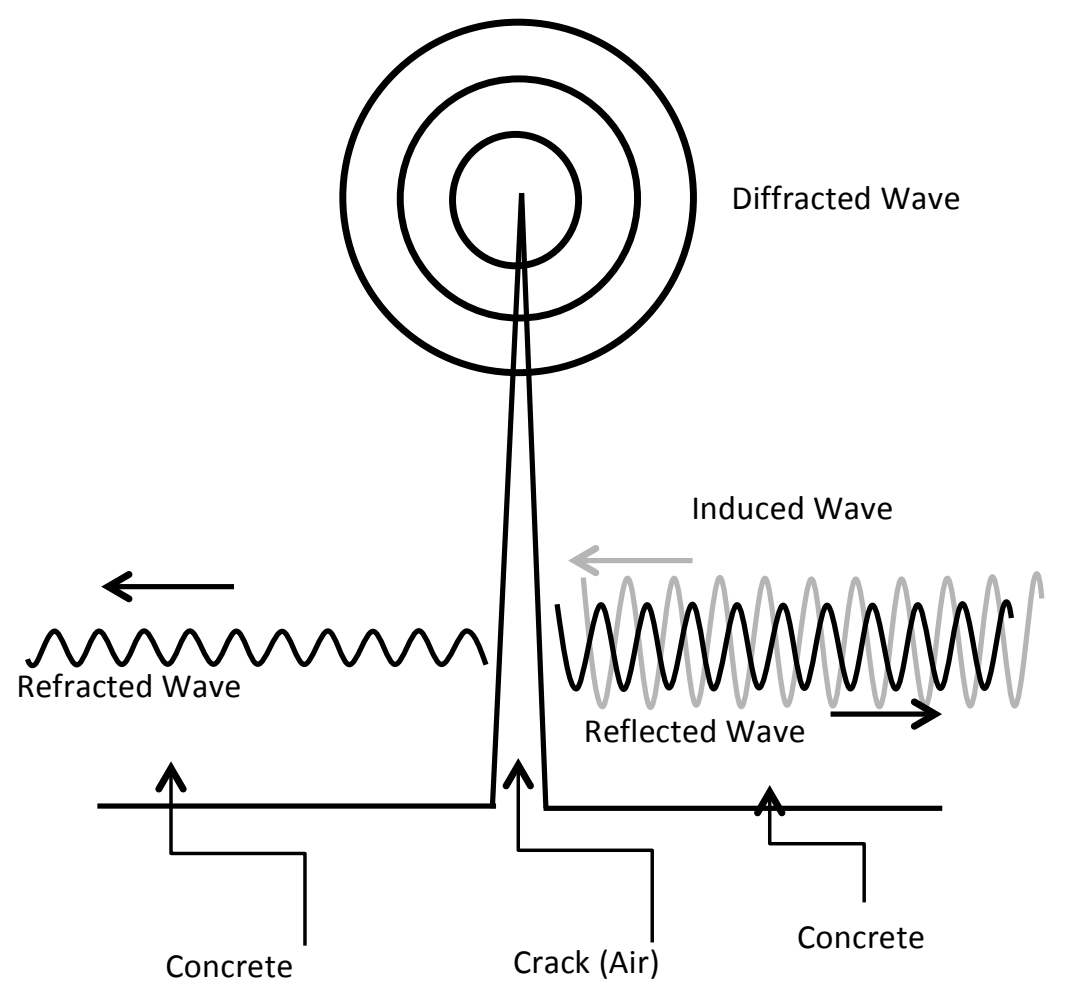

Figure 2.3: Mechanism for Diffraction, Refraction and Reflection of Stress Waves

If the acoustic impedance of the two materials is very similar, the energy of the refracted wave is precisely that of the induced wave. Although, as the impedance of the secondary material decreases, with respect to the first material, the more of the wave's energy that will be reflected, causing a large loss in transmissibility. At the concrete air interface the majority of the induced wave is diffracted and reflected, and is the premise of most ultrasonic non-destructive techniques. 


$$
\begin{aligned}
& A_{\text {Reflected }}=A_{i} \frac{Z_{2}-Z_{1}}{Z_{2}+Z_{1}} \\
& A_{\text {Refracted }}=A_{i} \frac{2 Z_{2}}{Z_{2}+Z_{1}}
\end{aligned}
$$

The amplitudes of the reflected, and refracted, waves are given by Equations 2.5 and 2.6 respectively (Sansalone $\&$ Streett, 2007). Here $Z_{2}$ is the acoustic impedance of the material the stress wave is transferred to, and $Z_{1}$ is the acoustic impedance of the material in which the stress wave is generated. At the concrete/air interface $Z_{1}$ represents the concrete's impedance and $Z_{2}$ is the acoustic impedance of air. As the acoustic impedance of air is much smaller than that of concrete the majority of the induced wave is reflected as a crack initiates through the signal's path.

\subsubsection{Factors Affecting Wave Propagation in Concrete}

Concrete provides many challenges for ultrasonic testing techniques. A non-homogeneous material causes a high rate of attenuation of any transmitted wave. The total attenuation is the summation of the absorption and scattering a stress wave experiences, and is commonly measured as loss in sound pressure per distance of travel. Scattering, in concrete, is a function of the maximum particle diameter; the smaller the particle the higher the scattering experienced by the signal. Attenuation caused by scattering, has a great impact on the signal response of the material. The effects of scattering and absorption will largely reduce the signal strength reaching the receiver; as well the spectral response period will increase, due to signal lag, and leading to skewed results.

Attenuation is primarily caused by the fine particles in the concrete matrix. These small particles scatter the transmitted signals. The attenuation of the signal becomes more apparent as the frequency of a stress wave increases. Large particles such as coarse aggregate are homogeneous in their nature and will facilitate the transfer of a signal within 
the concrete. The scattering and absorption of signal becomes a challenge when imperfections are far away from the transmitting or receiving transducers. As the resolution of the internal structure of the concrete material is the main challenge as it is a function of induced frequency. Where the higher the applied sample wave's frequency, the clearer the obtained image of the internal structure of the concrete member will be. Using these high frequencies causes the signal to attenuate rapidly, giving a poor representation of the internal structure. The attenuation of ultrasonic waves was found to be $2.86 \mathrm{~dB} / \mathrm{cm}$ corresponding to concrete's natural frequency (Rao and Lakshmi, 2003). This means that for every centimeter an ultrasonic pulse travels through concrete it loses approximately $50 \%$ of its energy.

\subsection{Applications of Embedded Piezoceramic Sensors in Concrete}

\subsubsection{Material Property Diagnostic}

The successful application of piezoceramics to actively monitor the Young's modulus, and Poisson's ratio of concrete was presented in the literature. This application quickly and effectively provides engineers with the placing condition of concrete after casting, as well as signs of deterioration throughout the life span of the structure, thus allowing for proper maintenance assessments to be carried out.

Surface mounted piezoceramic sensors have been successfully used in determining the two properties, Young's Modulus and Poisson's Ratio, of concrete (Li et al., 2009). In the study, the Reyleigh's, and longitudinal wave velocities were determined. Using the speeds of the waves, the Poisson's ratio, and Young's modulus could be back calculated using the known density of the concrete. Although the technology is valid and proved to give accurate results of the material properties, its field of application is limited. In order to give accurate results, these sensors can only be used on shallow members that have an accessible surface for piezoceramic patches to be mounted to, as well as a good mounting agent to ensure proper signal transmission. The application of surface waves has also been successfully carried out 
in characterizing the strength of concrete, although follows the same drawbacks (Gudra and Stawiski, 2000).

In the study presented by Wu et al. (1995), the drawback of surface mounted piezoceramic sensors was eliminated. In the study the authors used embedded piezoceramic sensors in order to run a similar analysis. The sensors used were specifically designed, as to properly isolate and protect the piezoceramic patch during concrete casting. Each sensor was made in such a way that it could receive the shear, and pressure wave for an induced impact. The impact was generated using a single burst square signal resembling an impact. This impact signal would generate a pressure, and shear wave within the concrete specimen, that were received by the second transducer. The received signal could be then separated into the two waveforms, via a bandwidth filter. Knowing the size of the emitting piezo patch the authors could calculate the generated frequencies that would constitute for the pressure wave, and the bandwidth that constituted the shear wave. Once the two waves forms were separated, the speed of each wave was computed, and using the equations of wave propagation and known density, the Young's modulus, and Poisson's ratio of the concrete could be determined. The embedded piezo patches proved to give very accurate results when compared with well-established methods such as the impact-echo, and the Pundit ultrasonic system; although they are much easier to implement as part of an infrastructure and eliminate the need for external equipment.

\subsubsection{Early-Age Performance Monitoring of Concrete}

Traditional methods of determining early strength of concrete structures primarily consist of destructive methods. Generally, standard concrete cylinders are cast and tested under compressive load providing the maximum load that the concrete can sustain at a certain age. In order to provide accurate and reliable results, the method requires an extensive number of compressive tests, and a large number of concrete cylinders that need to be cast from the same patch of the concrete used in casting the specimen. The destructive method may be effective for lab specimens, where a single specimen's early strength needs to be monitored; although in real world applications it becomes impractical. The vast number of 
samples necessary to get a clear understanding of the entire structure's concrete early strength becomes unfeasible, and very time consuming, limiting contractors to a few random samples taken out of the multiple concrete batches delivered on site. The cylinders will only provide a general representation of the entire structure's strength, and do not provide any representation of the quality of the concrete's placement, such as honeycombing. In recent years two major testing methods have emerged in order to give contractors the ability to monitor the early strength of multiple concrete members' within a building system.

The first method uses the heat of hydration generated by the specimen in order to give an indirect representation of the internal curing process. A thermocouple is embedded into the structure and provides data on the heat of hydration generated during the curing process of the concrete member (Ayotte et al., 1997). The heat of hydration is found to diminish through time, and can be correlated to the strength gained by the member. The analysis will provide what percentage of the desired concrete strength is achieved, and not provide a means of determining an exact value for the concrete's strength. A similar analysis was carried out using fiber optic where the concrete performance can be continuously monitored (Lin et al., 2004). The two methods provide a vague representation of the early life performance of the concrete, although being indirect; they are not accurate to the true condition of the member, nor economically feasible.

The second method is ultrasonic based and was found in the literature to consist of two categories of signal processing. Monitoring ultrasonic wave propagations in concrete is key information that can be used to determine the performance of the concrete. Since the physics behind ultrasonic wave propagation is largely governed by the internal structure of the medium, monitoring of the early concrete performance can be conducted (Voigt et al., 2006). The first category of signal processing relies on the properties discussed in Section 2.1.2 of this report; the velocity of an ultrasonic wave is proportionate to the density, modulus of elasticity, and Poisson's ratio of the material. By knowing the velocity of an ultrasonic pulse, and the density of the material this stress wave is traveling through, the characteristics of the concrete can be accurately determined. 
The second category of signal processing for the ultrasonic method relies on peak amplitude analysis of harmonic excitations. The embedded actuators are excited to various harmonic frequencies, and the amplitude of the wave is recorded from the receiving transducer. The peak amplitude of the frequencies is then recorded in order to identify the compressive strength of the object. It is found that the peak amplitudes of the received harmonic waves diminish rapidly within the first seven days of concrete curing, just as the compressive strength of the concrete increases rapidly (Song et al., 2008). The method takes advantage of the embedded piezoceramic sensors that are used in property diagnostic, and does not require additional equipment, making it the preferred method for early-strength monitoring of concrete.

The analysis can be carried out using the wave propagation of a one-dimensional longitudinal wave that can be expressed in Equation 2.7. $c_{b}$ is the wave speed, $\mathrm{E}$ is the modulus of elasticity and $\rho$ is the density of the material (Achenbach, 1973). Thus Equation 2.8 , represents the power of the corresponding wave where $\omega$ is the angular frequency, and $A$ is the amplitude of the wave.

$$
\begin{gathered}
c_{b}=\sqrt{E / \rho} \\
p=\frac{E A^{2} \omega^{2}}{2 c_{b}} \\
A=\left(\frac{1}{\omega}\right)\left(\frac{4 p^{2}}{E \rho}\right)^{0.25}
\end{gathered}
$$

Rearranging the two equations, the amplitude of the wave can be seen to be directly proportionate to the modulus of elasticity of the material, Equation 2.9, and the relationship is very important in correlating the early strength to the wave propagation (Song et al., 2008). As concrete cures, and gains strength, the modulus of elasticity of the material increases rapidly thus diminishing the amplitude of the wave. 


\subsubsection{De-Bond of Reinforcement}

De-bond detection in reinforced concrete member is a very good application of embedded piezoceramic sensors for condition assessment of reinforcement bars in reinforced concrete structures. An arrangement of piezoceramic patches was attached to the flexural reinforcement that was then embedded into concrete ( $\mathrm{Wu}$ and Chang, 2006). The reinforcement bars were designed to have de-bonding defects of varying lengths as to study the effect of de-bonding on an emitted ultrasonic signal through a steel medium. It was determined that the higher the de-bonding between concrete and steel the higher the amplitude for the received wave. The reason for this is the lack of interaction between the concrete and the steel. The interaction surface between the steel and concrete will absorb a large amount of wave energy and thus reduce the transmitted wave's energy. The study showed that the reflection of a wave traveling through steel is very small when in good contact with concrete; as the refraction of the wave is govern by the acoustic impedance of the material (Wu and Chang, 2006). The study proved that the wave's amplitude was affected exponentially to the de-bond length. Using the knowledge that de-bonding of reinforcement is similar to having voids within concrete; this increase in amplitude can be used to analyze the internal structure of concrete for multiple defects and is the basis of shear wave tomography.

\subsubsection{Impact Detection}

Collisions occurring from over height trucks with overpasses or ships with offshore oil platform structures are a very real threat. Without an active monitoring system, such an event could take years before it comes to the attention of engineers. If an active monitoring system could be implemented, it would provide engineers with the exact time of occurrence of the event, as well as the magnitude of the impact on the structure (Song et al., 2008). The information would allow maintenance crews to specify the location of various regions of deterioration within the structural members with a satisfactory level of efficiency, and the recommendations for the damaged regions could be decided to maintain safe and economic operation of the structure under investigation. 
Several systems have been proposed over the years, including accelerometer based, force based, and piezoelectric impact detection methods. The application of piezoceramic sensors is the least expensive method proposed in literature and was found to have a highly-linear response, making it ideal for load calculations following an impact (Song et al., 2008). Lead zirconate titanate has a linear piezoelectric voltage constant relating the applied force on the sensor to the output voltage observed. The relation can be expressed in Equation 2.10:

$$
V=\frac{g_{33} F t}{A_{\text {piezo }}}
$$

where $A_{\text {piezo }}$ is the surface area of the piezoceramic patch, $F$ is the applied force, $t$ is the thickness of the sensor, $\mathrm{V}$ is the output voltage generated, and $g_{33}$ is the piezoelectric voltage constant. Equation 2.10 considers the impact in the direction of polarization of the element. This means that the orientation of the piezoceramic element is highly important in determining the internal pressure induced by an impact. The piezoceramic constant can be selected based on the axis of the induced force or strain, and the direction of the polarization. In this case the 33 subscript means the element is polarized on the $3^{\text {rd }}$ axis and the force is applied on the $3^{\text {rd }}$ axis.

\subsubsection{Health Monitoring}

Many new and innovative apparatuses have been developed to facilitate the process of non-destructive health monitoring. These new testing systems all converge to two common goals: to reduce the time and cost of maintenance, and to improve safety. The traditional methods of non-destructive testing require on site persons that are highly experienced, and prove to be time consuming and very expensive. To eliminate the drawbacks of these traditional methods, development of permanent active monitoring systems has become a very desirable field. The implementation of fiber optic sensors for detection, and failure analysis has revolutionized the process of active health monitoring. The sensors have been 
successfully implemented in various RC structures. The sensors are very good for local monitoring although, the sensors gauge length governs their applicability. The sensors also come at a very high price, and are extremely fragile, further limiting their application. The field of piezoelectric embedded sensors is slowly emerging as a sufficient alternative to the fiber optics active monitoring system. This new innovative methodology provides a simple and inexpensive approach that allow for global monitoring of a structure, providing engineers with quick and accurate representation of the internal structure of reinforced concrete members.

The piezoceramic sensors have multiple variations of the signal analysis, depending on the application. In concrete non-destructive testing the primary methods used are: impedance based health monitoring, and the vibration-characteristics approach. The impedance based approach utilizes the admittance signature of the material in order to determine any abnormalities in the concrete (Annamdas et al., 2010). The admittance signature is recorded for any specific material, in this case concrete. The obtained result has to be done over the frequency range that will be used over the entire lifespan of the structure. The operating principal for this sort of system is that any change in the signature of the concrete will identify the appearance of damage in the specimen. In the study presented by Annamadas et al. in 2010, the effects of concrete spalling, and the introduction of saw cuts, were effectively captured by the sensors. Here the RMSD (Root Mean Square Deviation), from the signature was calculated. The value of the RMSD was seen to increase with the increase in damage existing in the concrete specimen. The method requires a single sensor in order to monitor a relatively large area, and could prove to be rather ineffective in large concrete members. The method relies on a bulk area of concrete, and was not found in the literature to have promising results for the cracking behavior of the concrete members. Rather the method provides engineers with a sense that something is wrong, not specifically what it may be.

The second methodology of piezoceramic sensors analysis in non-destructive testing is the vibration-characteristic approach. The vibration characteristic approach studies the localized damage of a predetermined array of sensors within a concrete specimen. Wang et 
al. (2001) have applied this method successfully for the investigation of debonding and delamination of concrete from the reinforcement bars, although its application to concrete structural health monitoring is relatively new territory. Song et al. (2004) successfully established a relationship between the appearance of cracking in a concrete bent cap, and the influence on a transmitted signal in the concrete specimen. The research was conducted using 4 embedded piezoceramic patches near the face of the specimen to monitor the appearance of shear cracking. The system proposed by Song et al. 2004 used a patch as a transmitter and a patch as a receiver. By alternation the receiver and transmitting, and recording reading for every combination of piezoceramic patches embedded the authors were able to establish a crack detection system.

The principle for the piezoceramic vibration characteristic system, relies on a sweep frequency that is implemented into the testing medium. This sweep frequency is decomposed either using a Fourier transform or Wavelet transform into a frequencyamplitude spectrum. The spectrum is then analyzed using the normalized Root Mean Square Deviation (RMSD) as presented in Equation 2.11 (Sun and Yan, 2008).

$$
\operatorname{RMSD}(\%)=\sqrt{\frac{\sum\left(E_{i j}-E_{h j}\right)^{2}}{\sum E_{h j}{ }^{2}}}
$$

where $E_{i j}$ and $E_{h j}$ are the current, and healthy state energies, respectively, of a specific frequency $\mathrm{j}$. The difference between the healthy state energy and the damaged state energy of each sampled frequency are calculated and divided by the Root Mean Square (RMS) of the healthy state spectrum. The calculated RMSD is a representation of the loss in transmissibility due to damage between two piezoceramic patches. A RSMD value of 0 represents a healthy non-damaged section, where a value of 1 represents a complete interruption of the transmissibility or member disintegration. The literature presents multiple successful application of the RMSD method. The topic is presented in more detail 
in Chapter 4 of the current study, as it is the basis of the research from here in and the foundation of the development of the active health monitoring system proposed.

\subsection{Alternative Non-Destructive Techniques}

\subsubsection{Health Monitoring Using MIRA 3D Tomographer}

The MIRA tomographer is a nondestructive system capable of detecting multiple flaws within a concrete sample, and gives a $3 D$ representation of the internal structure. The system is composed of two components, an antenna array, and a computer. The antenna array is composed of 40 low frequency dry point contact transducers arranged in 10 rows of 4 transducers each, and is presented in Figure 2.4. The antenna array uses the "pitch-catch" method, illustrated in Figure 2.5. These dry contact transducers eliminate the need for a contact medium to the concrete, and are spring loaded to allow the use of the array on very rough surfaces while still providing accurate results.

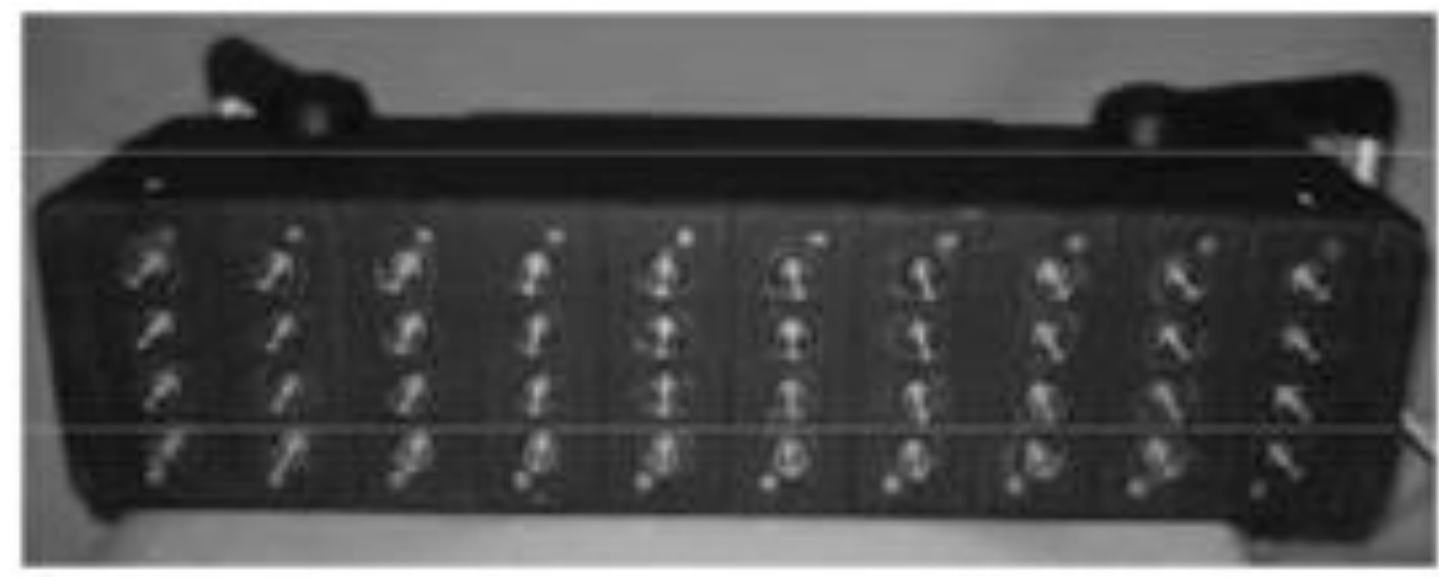

Figure 2.4: MIRA Tomographer's Transducer Array (Germann Instruments, 2010)

The antenna itself operates in a very similar fashion to other pitch-catch instruments. The first row of transducers will implement a stress wave into the test element while the other 36 transducers receive the signal. The second cycle would involve the second row of transducers emitting the signal while the remaining 32 probes receive the signal as demonstrated by Figure 2.5. The process is repeated until each of the 9 rows has 
transmitted a signal. The whole process takes less than 3 seconds to complete along with the data acquisition, and data processing.

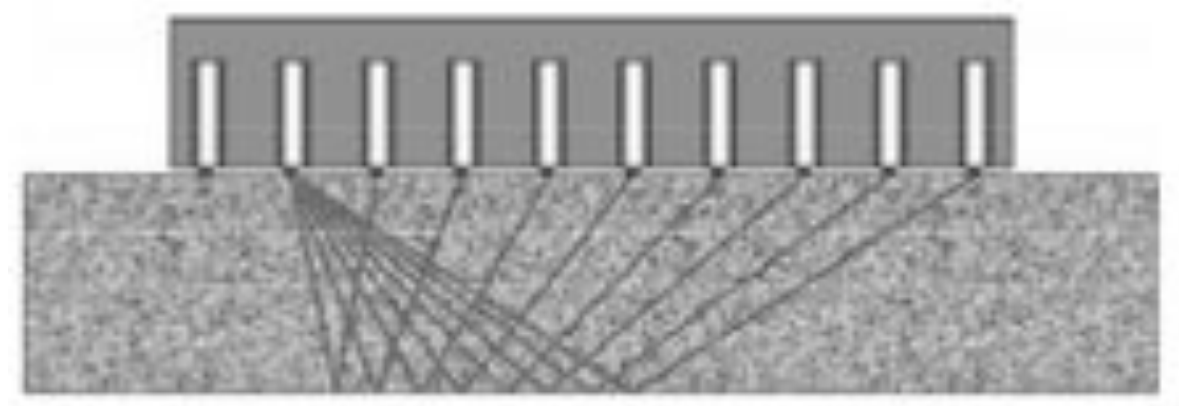

Figure 2.5: Signal Flow Using MIRA System when the Second Row of Transducers is Emitting Signal (Germann Instruments, 2010)

For signal processing, the antenna is wirelessly connected to a host computer. The preloaded software uses what is called Synthetic Aperture Focused Technique or SAFT. The signal processing tool helps to clarify the signal of distortions due to the inhomogeneity of the concrete itself. The primary goal of the software is to determine the depth of any imperfections the signal may encounter. The software uses Equation 2.12 (Germann Instruments, 2010) to determine the depth of the reflection. Figure 2.6 demonstrates the meaning of the variables where " $\mathrm{t}$ " is the time of reflection, " $\mathrm{d}$ " is the depth of the reflection medium, and " $\mathrm{C}$ " is the wave speed within the test material.

$$
d=C \frac{\Delta t}{2}
$$
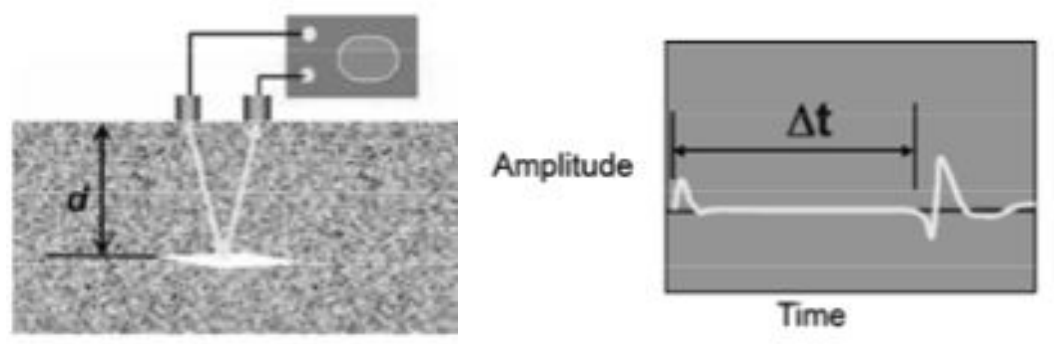

Figure 2.6: Transient Wave Response (Germann Instruments, 2010) 
In order to provide the user with an accurate three-dimensional representation of the internal structure of the test specimen, the software has to accurately map the position of the reflected signals. It is able to achieve this by spatial averaging of the reflected signals. The computer uses a complex mathematical algorithm to accurately map these points and create a three dimensional image. These three dimensional images are represented on three orthogonal planes denoted as C-Scan, B-Scan and, D-Scan. These planes are illustrated in Figure 2.7 on a three dimensional representation taken from the MIRA's user manual. The information on these three planes allows the operator to look at any one specific plane within the scanned test specimen.

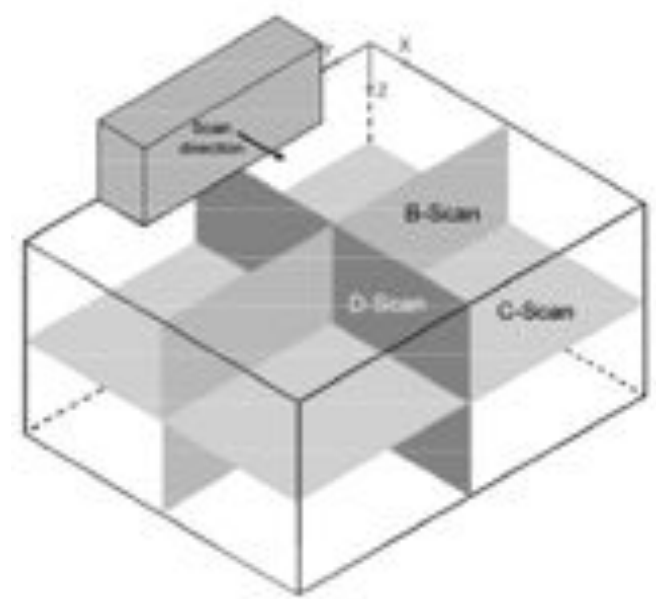

Figure 2.7: Orthogonal Planes as Represented with the MIRA (Germann Instruments, 2010)

\subsubsection{Fiber Bragg Grating Fiber Optic Sensors}

Fiber Bragg Grating (FBG) fiber optic sensors have emerged in recent years as a new form of strain gauges that can be embedded into various materials. The fiber optic cables have been successfully applied in various mechanical sensing applications, including civil structures. Fiber Bragg Grating is one of the multiple fiber optical sensing systems available on the market today. The sensors work by measuring the change in wavelength when strain is applied to the cables, Rizk el al. (2012). This change in wavelength can then be correlated to the strain experienced by the cable itself. The sensors are very effective for sensing the appearance of cracking in reinforced concrete members, and were found in the literature to provide very accurate strain readings. The operating wavelength, or Bragg wavelength, can 
range from 1530-1550 nm. FBG sensors have the advantage of multiplexing, making them ideal for concrete health monitoring. Multiplexing allows for multiple sensors to be installed onto one continuous cable that can be arranged in the concrete matrix. This is achieved form the manufacturing process of the cables, where each sensor can be adjusted to have a different initial wavelength. The FBG sensors can then be identified based on its design wavelength.
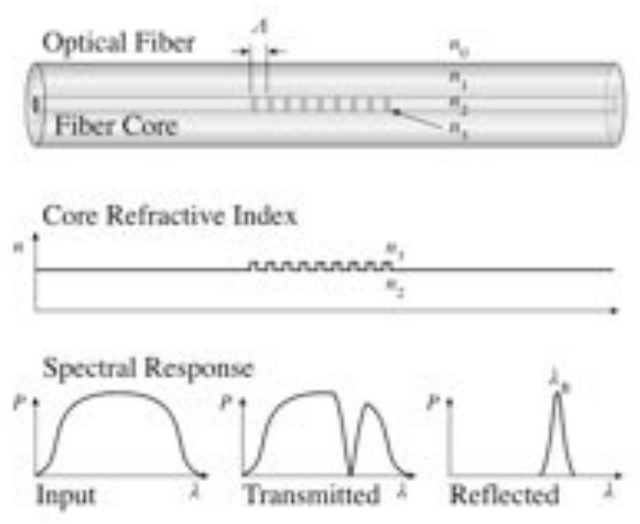

Figure 2.8: Transmission and Reflection Spectra of a Fibre Bragg Grating (Rizk el al. 2012)

A fiber Bragg grating is wavelength-dependent filter/reflector $\left(n_{3}\right)$ formed by introducing a periodic refractive index structure $\left(n_{2}\right)$, with spacing $(\Lambda)$ on the order of a wavelength of light, within the core of an optical fiber. Whenever a broad-spectrum light beam impinges on the grating, it will have a portion of its energy transmitted through, and another reflected off $\left(\lambda_{B}\right)$ as depicted in Figure 2.8.

\subsection{Crack Spacing and Width Calculations}

The Canadian CSA-S474(2004), and the Norwegian NS 3473 E-1992 building codes were found in the literature to provide similar approaches for the crack width and crack spacing calculations. The two codes were also found to provide the most accurate results for the cracking behavior of reinforced concrete members (Dawood and Marzouk, 2010). Both the Canadian and Norwegian codes models were adopted in the research to verify the results obtained from the experimental program, as well as establish the location of the proposed piezo-sensors' within the reinforced concrete matrix. 
The prevision provided in CSA-04 dictates that the average crack spacing can be calculated based on Equation 2.13. The equation takes into effect the geometry of the arrangement of reinforcement in the reinforced concrete member, as well as the effect of bond stress and strain gradient, as illustrated in Figure 2.9. The reinforced member's geometry is taken into account by the $2(C+0.1 S)$ term, where $C$ is the concrete cover, and $S$ is the spacing between adjacent reinforcement bars. The bond stress and strain gradients are taken into account by the $k_{1} k_{2} d_{b e}^{\prime} h_{e f} b / A_{s}$ term. Here $\mathrm{k}_{1}$ is the bond properties coefficient taken as; 0.4 for deformed bars, or 0.8 for plain bars. The $k_{2}$ coefficient is representative of the strain gradient and is dictated by Equation 2.14, where $\varepsilon_{1}$ and $\varepsilon_{2}$ are the smallest and largest tensile strains in the effective embedded zone. The geometry of the member is accounted for where; $d^{\prime}$ be being the bar diameter; $h_{e f}$ is the effective embedded thickness; $b$ is the base width of the section; and $A_{s}$ is the area of reinforcement found in the effective thickness, as shown in Figure 2.9. Where $a_{1}$ is the distance to the top of the outer layer of steel reinforcement, and $a_{2}$ is the distance to the inner layer of reinforcement.

$$
\begin{gathered}
S_{m}=2(C+0.1 S)+k_{1} k_{2} d_{b e}^{\prime} h_{e f} b / A_{s} \\
k_{2}=0.25\left(\varepsilon_{1}+\varepsilon_{2}\right) / 2 \varepsilon_{1}
\end{gathered}
$$

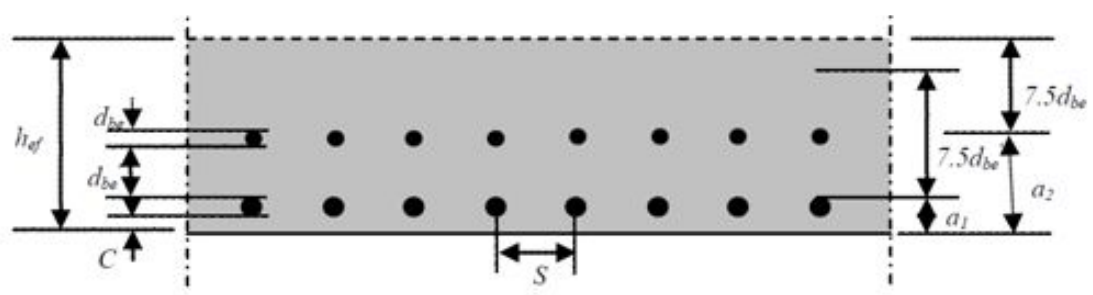

Figure 2.9: Effective Embedment Thickness (CSA S474 2004 Code)

The Canadian code suggests that the crack width is a function of the average tensile strain $\left(f_{t}\right)$, given by Equation 2.15, multiplied by the average crack spacing $\left(S_{m}\right)$. In Equation 2.15, $\varepsilon_{1}$ is the principal tensile strain of the tensile reinforcement and $f^{\prime}{ }_{c}$ is the concrete's compressive strength. 


$$
\begin{gathered}
f_{t}=\left(0.33 \sqrt{f_{c}^{\prime}}\right) /\left(1+\sqrt{500 \varepsilon_{1}}\right) \\
w_{m}=r \varepsilon_{1} S_{m} \\
w_{k}=1.7 w_{m} \\
r=1-\frac{\beta}{2.5 k_{1}}\left(\frac{\sigma_{s r}}{\sigma_{s}}\right)^{2}
\end{gathered}
$$

The previsions provided by the Norwegian Code NS-474E-92 have a different approach for calculating the crack width that accounts for the tensioning stiffening effect. The effective concrete area in accordance to the Norwegian Code NS-474E-92 is presented in Figure 2.10, where $\Phi$ represents the diameter of the steel reinforcement. The average crack width $\left(w_{m}\right)$ is calculated using Equating 2.16 where $\varepsilon_{1}$ is the principle strain of the tensile reinforcement, $S_{m}$ is the average crack spacing and $r$ is the factor accounting for the tensioning stiffening effect. The $r$ factor is calculated using the stress in the reinforcement at the cracking load $\left(\sigma_{s r}\right)$ and applied load $\left(\sigma_{s}\right) . \beta$ is the factor taking loading procedure into account, and $k_{1}$ is the reinforcement influence factor, taken as 0.4 for ribbed bars. The maximum crack width $\left(w_{k}\right)$ can be calculated using equation 2.17.

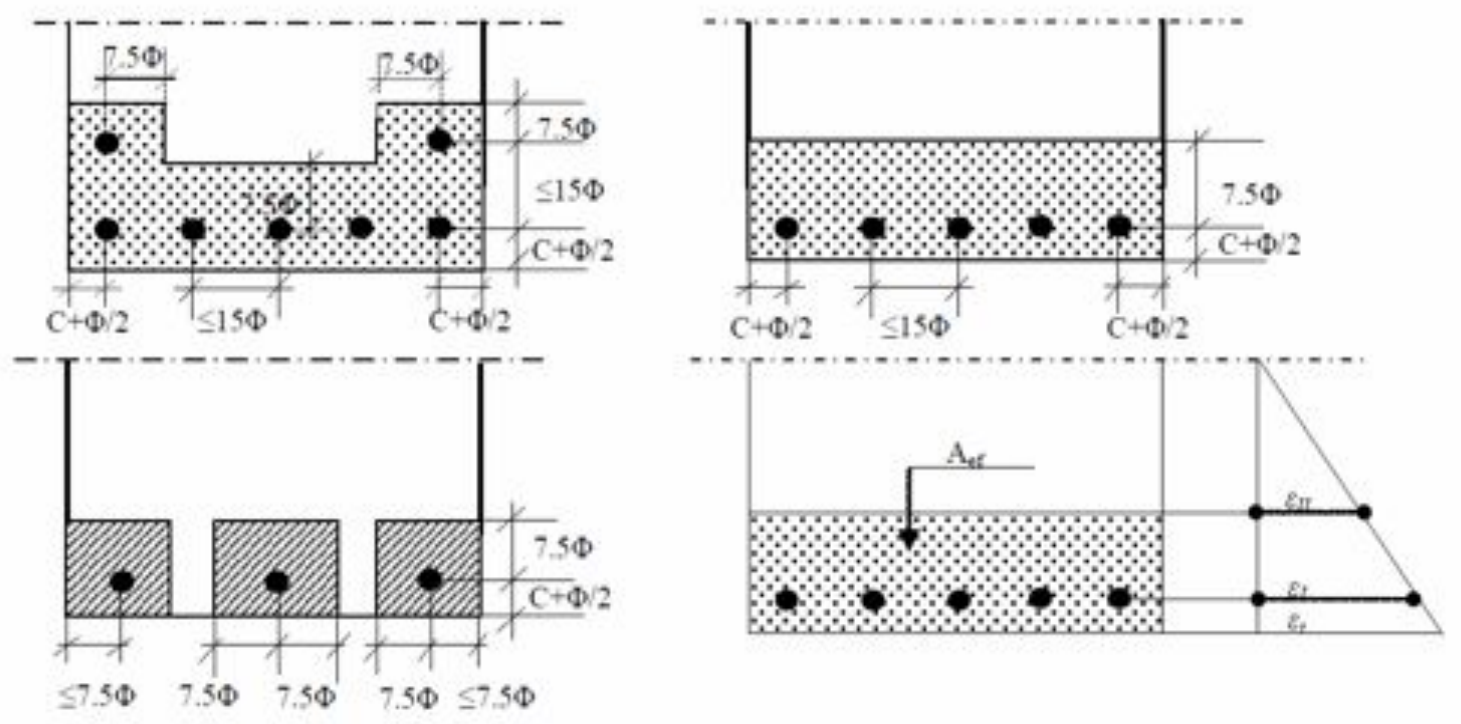

Figure 2.10: Guidelines for Determination of Effective Concrete Area for Calculation of Crack Spacing (Shaded) [NS 3473 E 1992Code] 



\section{Chapter 3 MIRA 3D Tomographer}

The shear wave tomographer is a state of the art equipment that has multiple applications in the non-destructive evaluation of reinforced concrete members that include determining: the location of pre-stressing concrete tendons, the location of steel reinforcement, delamination of structural components, reinforcement de-bond, etc. In the following chapter an investigation was carried out to verify the equipment's capabilities. The equipment was also applied to determine the punching shear crack angle of thick concrete slabs. The chapter will begin by describing the investigation carried out for determining the maximum depth of signal penetration of the equipment, and the study of how the amount of reinforcement affects this, followed with the MIRA's ability to determine delamination of a external structural bearing wall, and its grouting condition. After establishing the 
equipment's capabilities, the study that was conducted to determine the punching shear angles will be presented.

The abilities of the shear wave tomographer would need to be determined and defined before conducting any sort of testing. The process of defining the abilities consisted of four

tests on various structural components at the Ryerson University's structures laboratory. These tests would be conducted on existing building components that are in service. In such way, the MIRA's capabilities can be verified on existing structural elements that are known to be in use and are part of an operational building.

From the four components, two of them consisted of regions in the laboratory that contain a very high reinforcement ratio to examine the effects of attenuation on the transmitted signal. The two samples that have a high reinforcement ratio were the structural floor of the laboratory as well as the structural wall of the lab. The remaining two samples would consist of load bearing walls within the structure, an exterior earth retaining wall in the basement of the laboratory and a wall sustaining the load.

The investigation into determining the punching shear crack angle was conducted on four thick concrete slabs. These slabs were located in the structural laboratory of Memorial University in St. John's, Newfoundland. The MIRA 3D tomographer was not found in the literature to be applied to locating and measuring punching shear cracks of thick slabs, making the investigation a new application of this equipment.

\subsection{Experimental Program for Verifying the MIRA's Capabilities}

The shear wave tomographer is known to have specific characteristics specified by its manufacturers. Although for the purpose of this section these properties would need to be verified. The most important of the specifications would be the depth of penetration of the ultrasonic wave. It is well known that concrete is a very difficult material to work with when it comes to ultrasonic testing due to its inconsistent composition. For this reason a test would need to be developed to determine the maximum depth of penetration. 

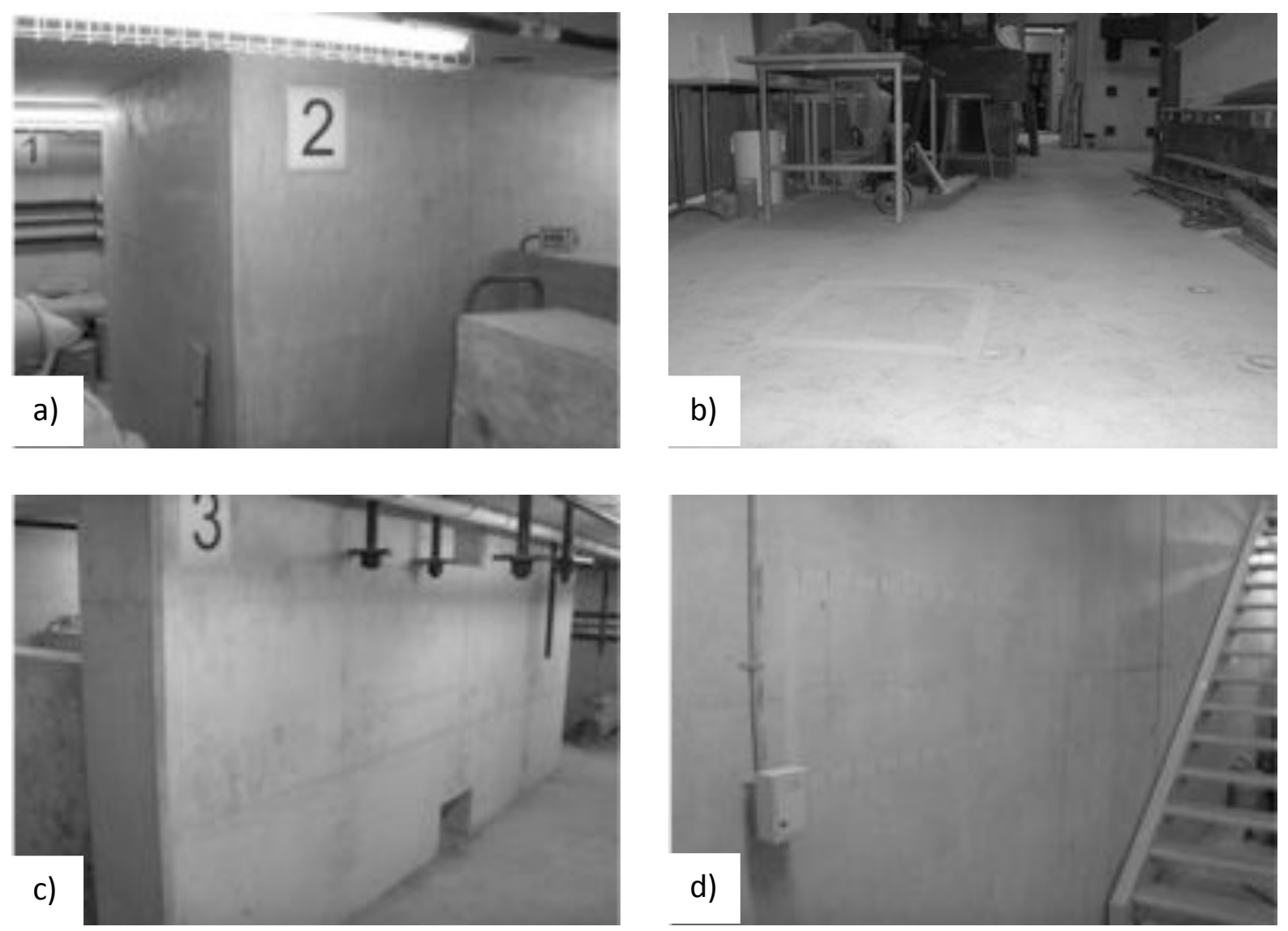

Figure 3.1: MIRA Test Specimens; (a) $2 \mathrm{~m}$ Deep Structural Wall; (b) $1.2 \mathrm{~m}$ Deep Structural Floor; (c) $0.4 \mathrm{~m}$ Deep Load Baring Wall; (d) $0.3 \mathrm{~m}$ Thick Exterior Basement Wall

\subsubsection{Depth of Signal Penetration and Effect of Reinforcement Ratio}

The depth of signal penetration, and effect of reinforcement ratios, was investigating using components of the structural floor in the structural laboratory of Ryerson University. Figure 3.1a demonstrates a thick concrete wall found in the structures laboratory. The wall has a depth of $2.0 \mathrm{~m}$ and thus would be ideal for determining, and establishing, if the maximum $(2.5 \mathrm{~m})$ specified depth of the MIRA tomographer can be achieved. Along with the $2.0 \mathrm{~m}$ deep wall, the structural floor of the laboratory has a thickness $1.0 \mathrm{~m}$ (see Figure $3.1 \mathrm{~b}$ ), existed in the structures laboratory was tested in order to establish a correlation between the depth of penetration and frequency of operation, as well as study the effect of high reinforcement ratios to the working condition of the equipment. 


\subsubsection{Reinforcement Location}

The shear wave tomographer is demonstrated to have a good capability of determining delamination as well as the position or reinforcement within a concrete specimen. In order to demonstrate these capabilities, the tomographer was used on a simple $400 \mathrm{~mm}$ thick structural wall (Figure 3.1c). The purpose of the test was to determine the location and spacing of reinforcement using the shear wave technology.

\subsubsection{Delamination and Grouting}

The capabilities of determining concrete de-lamination would be represented by scanning an exterior basement wall. The exterior basement wall would amplify the chances of finding this defect. The exterior wall is shown in Figure 3.1d. The insulation on the exterior of the wall along with the process of backfilling would certainly show the de-lamination output using the MIRA technique. According to the basic operation of the equipment, the smallest air pocket on the adjacent side of the test surface should show a change on the output of the software. This de-lamination would be seen if the back wall reflection is obtained that shows the thickness of the wall under investigation. Along with expecting de-lamination form the wall, it was noticed form observation that there was a section of the wall that was grouted after the completion of the wall due to a patch job. The patchwork would show what the effects of grout are on the output signal of the MIRA.

\subsection{Experimental Results for Testing the MIRA's Capabilities}

\subsubsection{Depth of Signal Penetration and Effect of Reinforcement Ratio}

\subsubsection{Structural Wall}

The test was done to determine the MIRA's maximum depth of penetration through a heavily reinforced concrete wall. The MIRA tomograph was used over a $500 \times 100 \mathrm{~mm}$ rectangle. A local control reading was taken at the lowest frequency of the MIRA. The depth 
of the highly reinforced wall is $2000 \mathrm{~mm}$. The test was conducted using a frequency of 20 $\mathrm{kHz}$. The output of the MIRA software is presented in Figure 3.2. As illustrated by the output the signal's maximum depth of penetration was $1800 \mathrm{~mm}$. At this depth a reflection of the signal can be seen, however, it is not providing any significant information about the interior of the wall. The significant signal penetration for this wall was $1200 \mathrm{~mm}$ since the signal beyond this depth is extremely weak and will not be very representative of the interior condition of the wall.

The image also illustrated a vague representation of reinforcement bars at a depth less than $200 \mathrm{~mm}$. The large wavelength makes it very difficult to get an accurate estimation of the bar size, although the image provides enough information to deduce that the bars are 50 $\mathrm{mm}$ apart. The result cannot be confirmed until structural drawings are provided to of the structural floor to verify the result

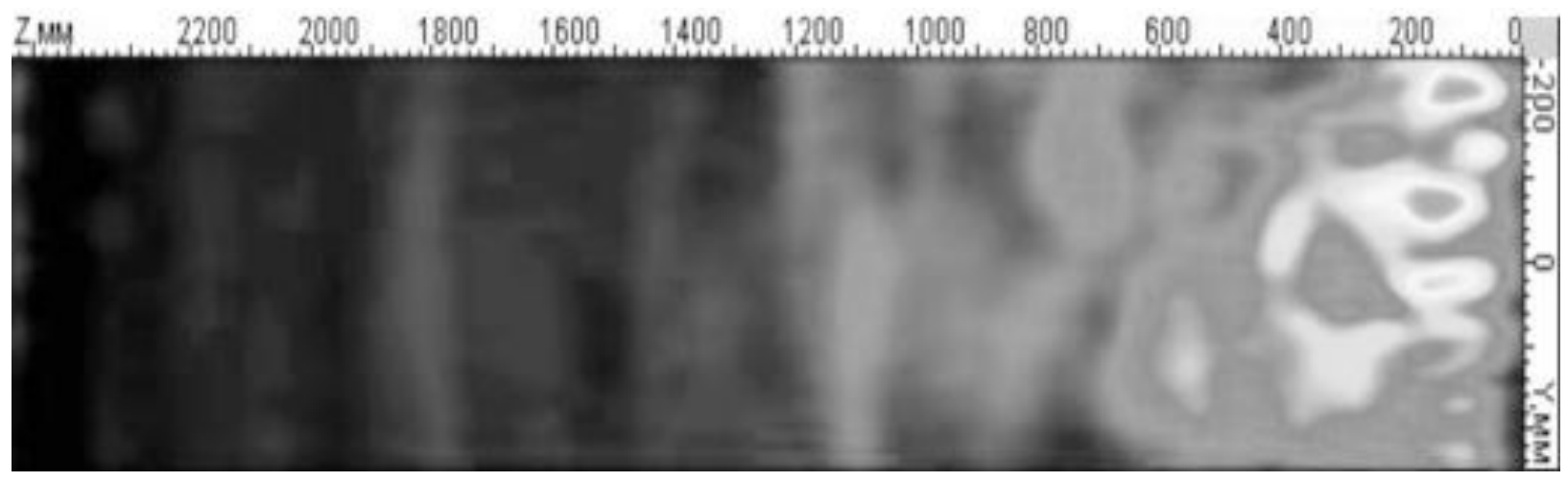

Figure 3.2: $2 \mathrm{~m}$ deep structural wall MIRA B-scan software output $20 \mathrm{kHz}$

\subsubsection{Thick Structural Floor}

The aim of this test was to determine the MIRA's effectiveness with thick, highly reinforced concrete slabs. The slab under investigation was constructed in 2004 , and there should be no signs of deterioration present. There are shafts that go through the slab and are spaced in a $700 \times 700 \mathrm{~mm}$ grid throughout the surface. In order to achieve meaningful results an area that was $500 \mathrm{~mm} \times 500 \mathrm{~mm}$ was mapped out over the thickness of $1000 \mathrm{~mm}$. 
The MIRA was found to be insufficient to give an accurate representation of the locations of reinforcement within the slab. The various B-scan images in Figure 3.3 illustrate the effects of changing the frequency of the antenna.

It is presented in Figure 3.3, the depth of penetration of the signal is largely influenced by the frequency used. The higher the frequency the less information was obtained on regions embedded deep within the specimen. At $20 \mathrm{kHz}$ the signal has good penetration well beyond a depth of $1.2 \mathrm{~m}$. The darker the image appears the less signal that is returned to the antenna, this becomes clear with frequencies equal to or greater than $50 \mathrm{kHz}$, the signal becomes almost insignificant beyond a depth of $800 \mathrm{~mm}$. Although from these higher frequencies we can get a better resolution of the interior, and more accuracy of the depth at which different features are placed within. The smaller wavelength of higher frequencies also helps to identify rather small defects within the slab.
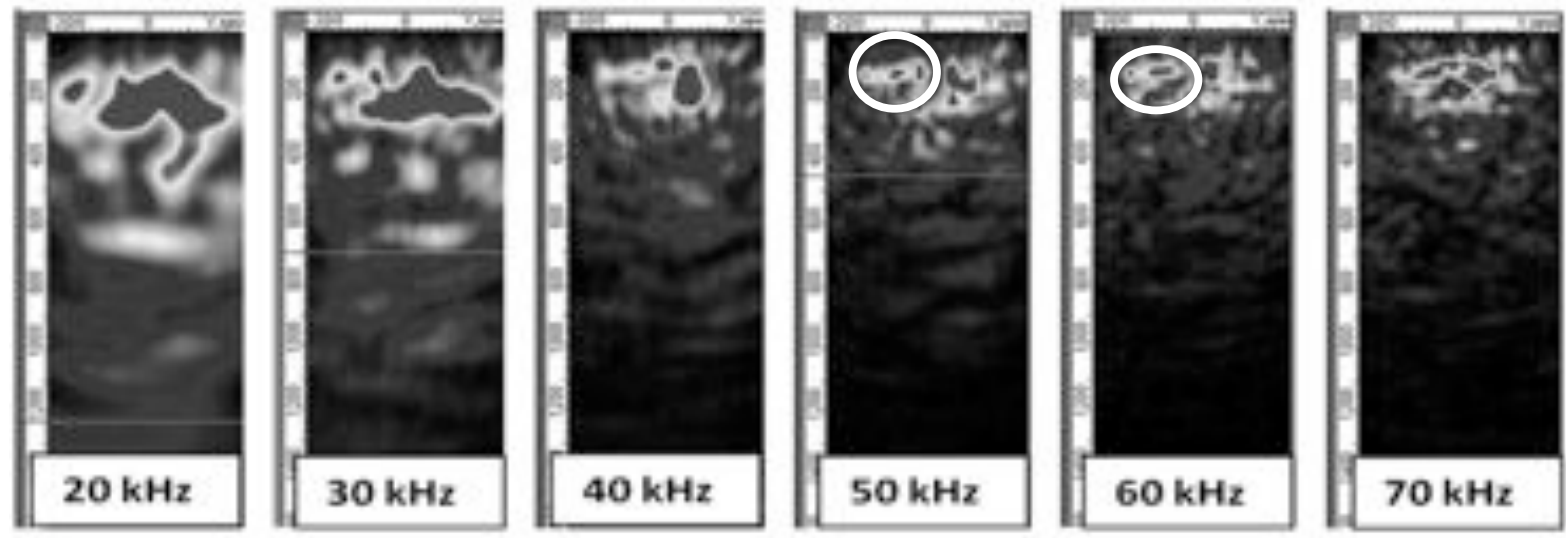

Figure 3.3: Deep structural floor MIRA B-scan software output $20 \mathrm{kHz}-70 \mathrm{kHz}$

The depth of the specimen, and the amount of reinforcement that is found inside, will greatly affect the information that can be gathered by the MIRA. This becomes evident in this scenario, as there is not enough information provided by these pictures in order to easily determine the placement of reinforcement within the slab. At frequencies of $50 \mathrm{kHz}$ and $60 \mathrm{kHz}$ reinforcement bars can be seen at a depth of $140 \mathrm{~mm}$ (marked with the white circles); although the image could be of voids or other imperfections, and no definite conclusion can be drawn from this reading due to an absent back wall reflection. 
The MIRA was successful in determining the depth of the slab. By using low frequencies, the ultrasonic pulses are less likely to get attenuated as they propagate through the slab, although they provide a less accurate representation of the internal condition. This observation can be seen when comparing the readings taken at $20 \mathrm{kHz}$ and $30 \mathrm{kHz}$.

We know that the slab has a depth of $1.0 \mathrm{~m}$, although the back wall reflection at $20 \mathrm{kHz}$ would suggest the slab is $1.1 \mathrm{~m}$ deep. The $30 \mathrm{kHz}$ reading that would suggest the exact slab depth of $1.0 \mathrm{~m}$ provides a much more accurate reading. The back wall reflection is lost at frequencies higher than $30 \mathrm{kHz}$, since the attenuation from the reinforcement within the slab, greatly decreases the signal strength.

\subsubsection{Determining Size and Location of Reinforcement}

The test was done to determine the MIRA's ability to determine the location of reinforcement within a $400 \mathrm{~mm}$ thick concrete wall. The MIRA tomograph was performed over a rectangle area of $500 \times 1100 \mathrm{~mm}$. A local control reading was done to easily point out the depth reading and location of reinforcement bars. For this test the machine was set to $40 \mathrm{kHz}$. The MIRA output is presented.

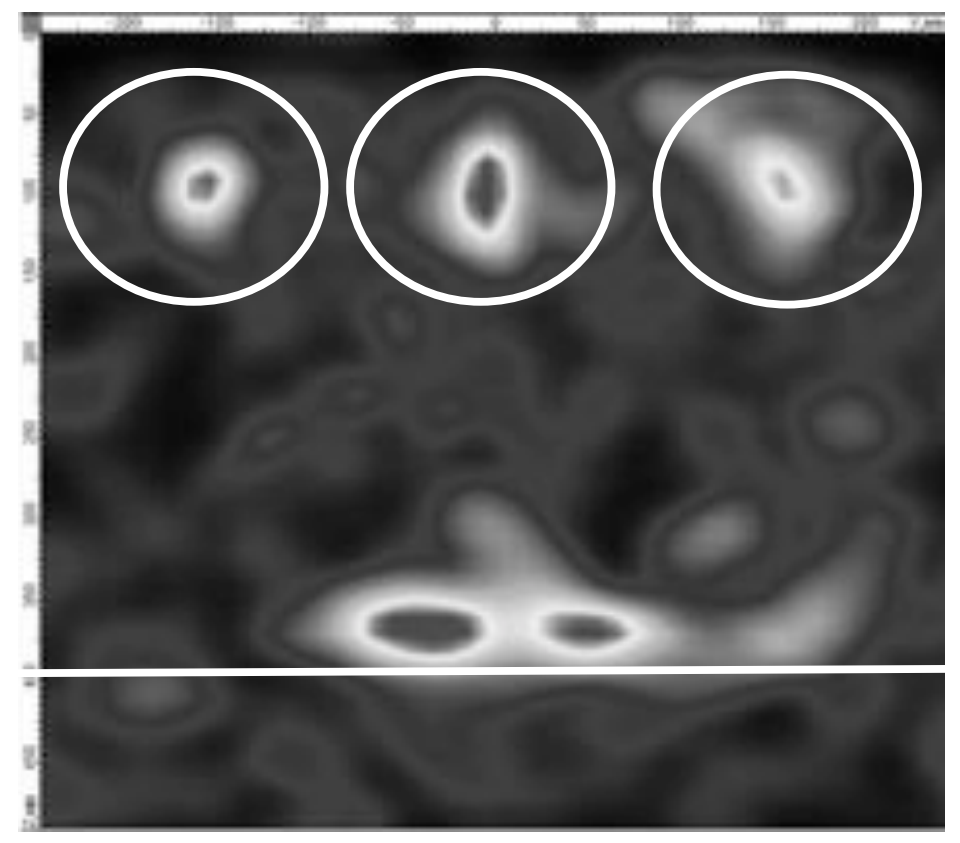

Figure 3.4: Basement Bearing Wall MIRA B-Scan software output $40 \mathrm{kHz}$ 
The three white circles at the top of the image identify the reinforcement. Reinforcement spacing of $150 \mathrm{~mm}$ was obtained between the white circles at $50 \mathrm{~mm}$ below the top surface of the structures. The fact that the reflections are reinforcement bars is confirmed by the absence of signal reflection from the back wall at position zero. Directly underneath the location of the middle rebar. There is also a slight reflection of the second layer of reinforcement underneath the middle bar at a depth of $350 \mathrm{~mm}$, although not clear due to the shadow created by the first reinforcement layer. The bar size can also be estimated to be $25 \mathrm{M}$. All the readings from this scan are estimations of what could be inside the bearing wall and cannot be finalized until structural drawings are used to confirm.

\subsubsection{Delamination and Grouting Condition}

The aim of the test was to verify the MIRA's ability to determine the thickness of the wall and the effects of crack repair on the signal output. The visible repair is over a $200 \mathrm{~mm}$ long region on the surface of the wall. To achieve meaningful results an area of $1200 \times 500 \mathrm{~mm}$ was mapped out over the $300 \mathrm{~mm}$ thick wall. The test setup is presented in Figure 3.5. For this test the machine was set to $50 \mathrm{kHz}$ and a step of $100 \mathrm{~mm}$ was established and 13 reading were taken.

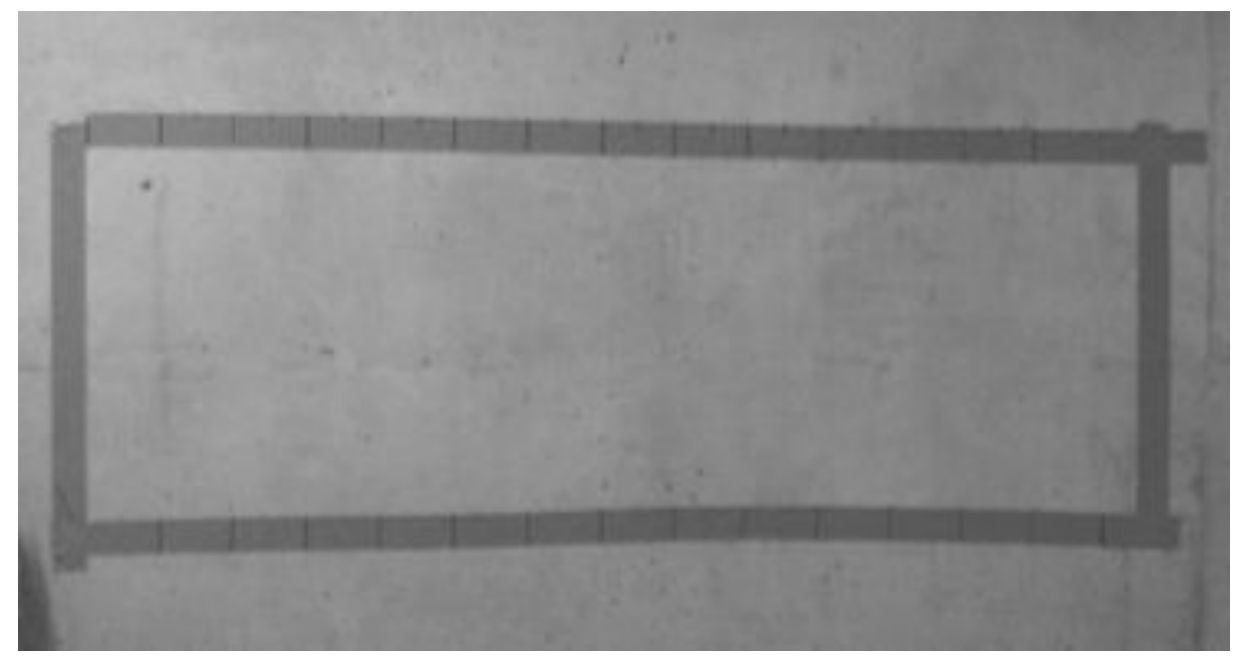

Figure 3.5: Basement Exterior Wall Test Setup 
Upon completion if the first reading at $50 \mathrm{kHz}$, the MIRA output in Figure 3.6 shows a poor surface condition, and cracking in the sample between steps 6-8 (white box).

Due to the low frequency used, the damage could not be correctly identified, or correctly positioned, thus a second reading at a higher frequency was taken. The higher frequency would provide a better representation of the top layer. Figure 3.6 also demonstrates that the machine was not calibrated properly, this is illustrated by the 10 equally spaced dots that appear as reinforcement near the top layer of the specimen. These are due to interference from surface waves. This kind of interference is common when the surface of the specimen has many voids and the machine cannot properly calibrate for these. Along with the dots, streaks can be seen on the top left image that would indicate the same problem.

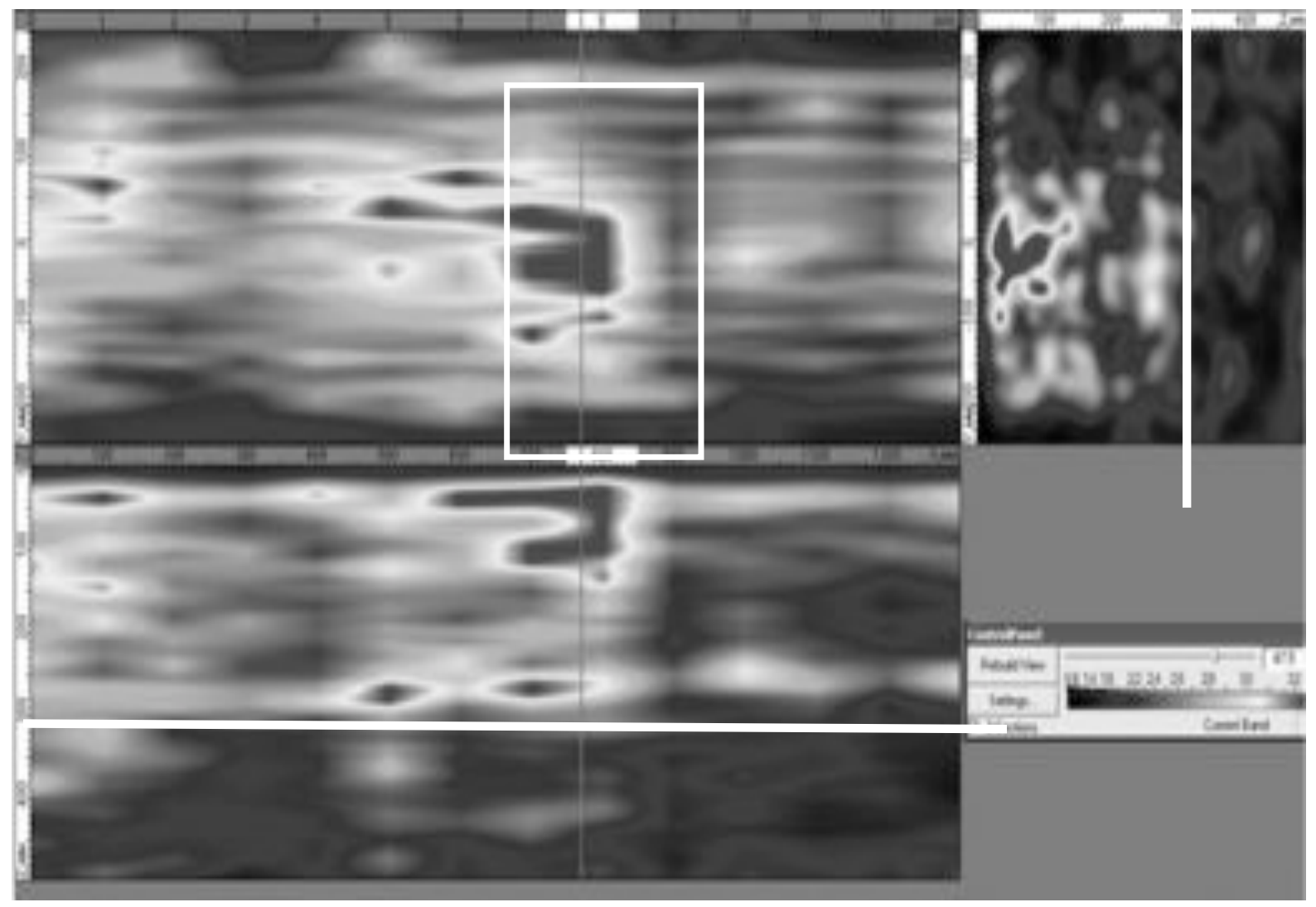

Figure 3.6: Basement Exterior Wall MIRA Software Output $50 \mathrm{kHz}$ 
Apart from the interference, the back wall reflection is visible and thus giving a good representation of the thickness of the wall; this is represented by the solid white line on the diagram. By having this back wall reflection present, the MIRA suggests that the wall is not in full contact with the soil on the other side. The output would be very similar to what is expected form the de-lamination of concrete specimen. Another reading of the same wall was taken using a frequency of $70 \mathrm{kHz}$, in order to better establish the damage detected at $50 \mathrm{kHz}$. The output obtained from the MIRA software at $70 \mathrm{kHz}$ is illustrated in Figure 3.7.

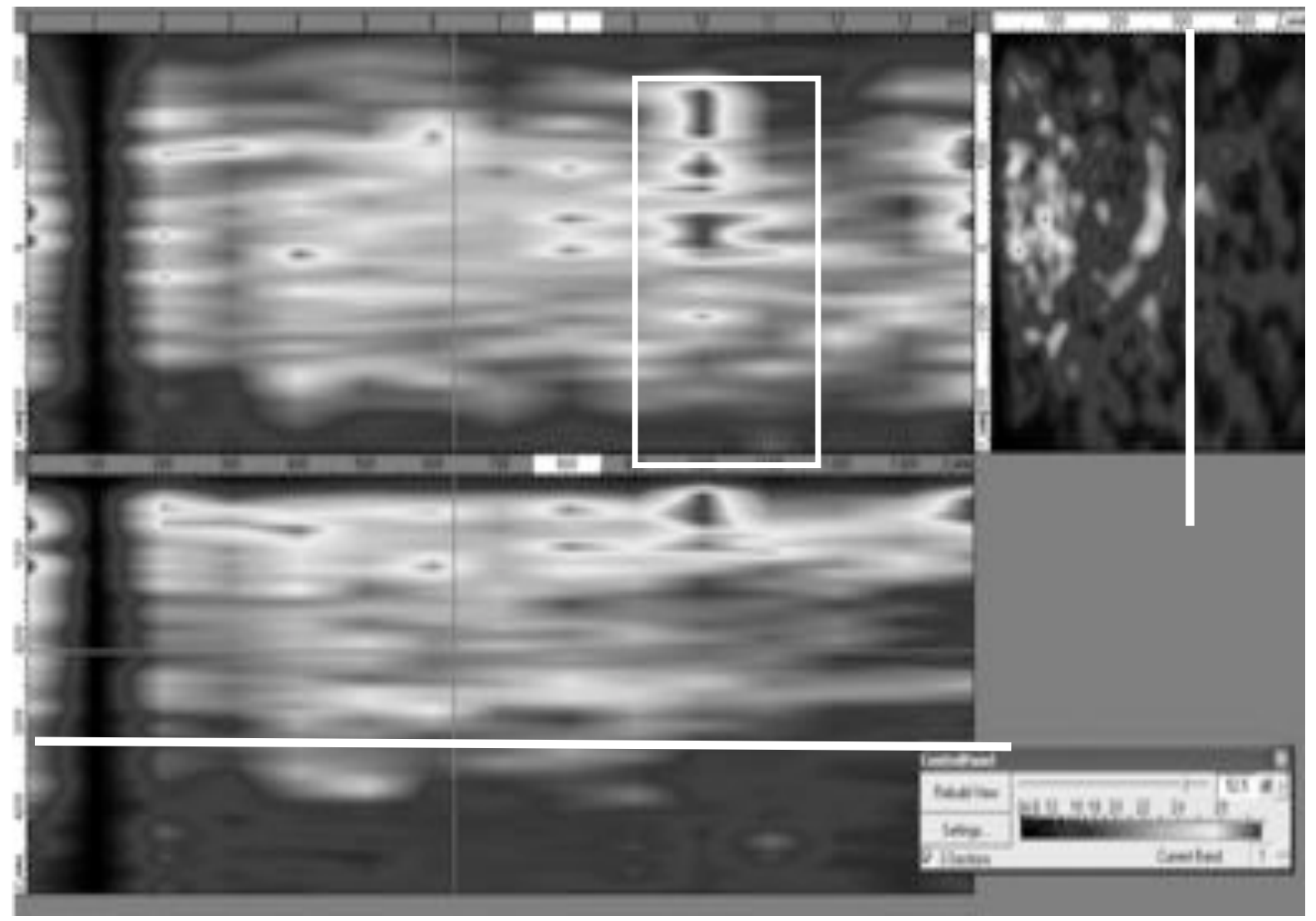

Figure 3.7: Exterior Basement Wall MIRA Software Output $70 \mathrm{kHz}$

The image corresponds to the back wall reflection found in the first reading of $300 \mathrm{~mm}$. Also the surface imperfections found in the first reading was confirmed by this image. When interpreting the test results the second position should be disregarded due to experimental error. Thus all the positions should be shifted over by one value to be interpreted. In the 70 $\mathrm{kHz}$ reading we can see the cracking discovered previously is not localized to the seventh to 
ninth position. This shows a consistent change in material properties in this region and can be concluded that there is cracking within the specimen. Although the interference of the poor surface quality is reduced, streaks can still be seen in the C-scan and are only reduced due to the relatively small wave length used for the reading. This is but far one limitation of the machine, although the interference can be diminished with higher degrees of calibration.

\subsection{Determining the Angle of the Punching Shear Crack for Deep Slabs}

The method of tomography has many applications in non-destructive testing of concrete specimens. For the purpose of this research, a series of tests were carried out in order to determine the MIRA's ability to determine the angle of the punching shear crack of a highstrength concrete slab. The following section demonstrates the experimental setup, and the results obtained upon completing the investigation using three-dimensional tomography.

\subsubsection{Experimental Program}

The punching shear crack angle would need to be determined for 4 different specimens. The four slabs were cast using high strength concrete. Three of the slabs had been loaded to failure, whereas the forth slab was loaded dynamically to $70 \%$ of its ultimate capacity. The slabs that were loaded to their ultimate capacity would prove to show the location and angle of the punching shear crack with relative ease. Once the failure punching shear crack would be determined, the results could be compared with those determined from the dynamically loaded slab in order to predict the location and angle of the punching shear crack that will form within this specimen upon failure. As well as determining a location for the crack and angle, the test would also demonstrate if there were any difference in the formation of the crack between the dynamic and static loading tests. Further information regarding the details and testing procedure of these slabs can be found in Rizk et al. 2011.

Slab 1 (Figure 3.8a) was the dynamically loaded slab, as described. The dimensions of the slab are $1900 \times 1900 \times 200 \mathrm{~mm}$. The slab was never tested to it's ultimate load, and thus 
can only give limited information on the formation of the punching shear crack, and where the shear crack would be expected to appear upon failure.

Slab 2(HS5) in Figure 3.8b was part one of the slabs that reached its ultimate capacity. The dimensions of the slab are $2650 \times 2650 \times 300 \mathrm{~mm}$. Due to its size relative to the MIRA tomograph, and the fact that it reached its ultimate capacity, the punching shear crack for this specimen should be clearly visible in the software output. The slab also has T-Headed shear studs, these would stiffen the slab and affect the angle of the punching shear crack, thus making the result different from that of the following two slabs.
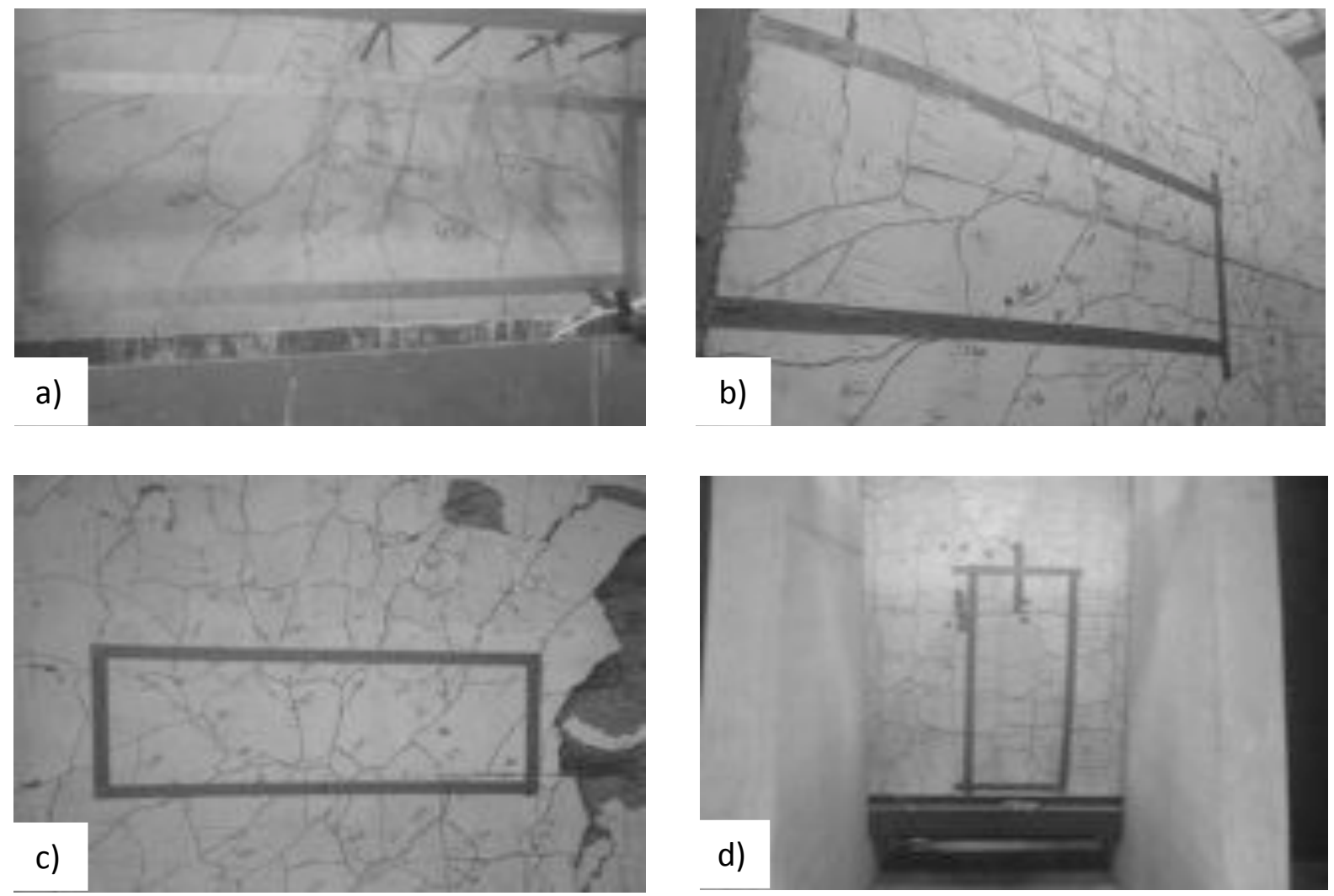

Figure 3.8: Test Surfaces of; (a) Slab 1; (b) Slab 2; (c) Slab 3; (d) Slab 4

Slab 3 (HS4) is shown in Figure 3.8c. The dimensions of the slab are $2650 \times 2650 \times 350 \mathrm{~mm}$. The slab was tested to ultimate load, and a large amount of surface cracking could be seen on the test surface. Unlike HS5 it does not contain T-Headed shear studs, thus we would expect a different angle for the punching shear crack. The slab is similar to Slab 4(HS7), presented in Figure 3.8d, and the results of these two slabs are expected to provide similar 
results. The difference between the two slabs is the depth where HS7 has a higher depth of $400 \mathrm{~mm}$ as compared to $350 \mathrm{~mm}$ in HS5. The different depth would show to have a minor effect on the way we interpret the results of the MIRA tomograph.

The four slabs are illustrated in Figure 3.8 in the condition they were found prior to testing. The tests were carried out without the aid of structural drawing, as no significant information could be gained from the slabs' details for locating the punching shear crack.

\subsubsection{Analysis Methodology}

In order to determine the angle of the punching shear crack, the known information would have to be eliminated from the image created by the MIRA software. The slab thickness was known in all the test cases, as well as the location of the column and surface opening cracks. By knowing the thickness of the slab the location of backwall reflection the signal can be filtered. This may not always be the case, although it is highly recommended to review engineering drawing of the test specimen prior to using the MIRA. Doing so will help to determine signal-noise that can render faulty results. This noise can be seen beyond the thickness of the specimen and should be rendered insignificant, as it is a transmitted wave reflecting in between multiple cracks within the specimen.

The software determines the time of transmission of the wave in order to determine the depth of the reflection. Signals that are reflected between cracks inside the slab will have a longer time of arrival and the readings would show cracks at a depth greater than that of the slab.

The next step would be to correlate between the different frequencies. Obtaining common reflections between multiple frequency readings suggests a common inconsistency in the material, and thus verifies that the reading obtained from an imperfection within the medium, and not due to noise. Once this was done the relative location of the reflections caused by the punching crack was known, as well as other cracks that would appear consistently for all readings. Correlating the software outputs between multiple frequencies is very important in highly damaged specimens. The process ensured that the reflection 
seen on the output are not due to noise caused by imperfections and are in-fact inconsistencies within the slabs.

After finding the general location of the punching shear crack, the column location needed to be determined within the software output. Once the location of the column was found in the image, one end point of the crack would be determined. Multiple readings showed surface opening cracks that are visible on the test surface. These surface opening cracks would pinpoint the other endpoint of the punching shear crack. This step was used for all the images that demonstrated these characteristics, as it made for a simpler analysis.

The next step would be to determine the signal that would reflect form the punching shear crack. This signal would appear as a back wall reflection within the depth of the slab. The reflection would happen at a greater depth than the location of the cracks. By drawing lines through the reflection, and correlating between the different frequencies, the results would consistently show two lines that intersect at an angle.

Upon determining the punching shear crack angle for the different frequencies for a particular slab, the values would be averaged to get the approximation of the real angle. Also, outliers were disregarded and are not part of the data set as described below for the particular slab

\subsubsection{Results}

The following section, analyses the results obtained by the software for the MIRA tomograph. The punching shear crack angle is also determined and presented for each of the four test specimens. Each test specimen consists of the numerous readings taken and the results are compared and contrasted.

\subsubsection{Slab 1}

Slab 1 was not tested to failure, yet it was put through dynamic fatigue. The purpose of using the MIRA on this specimen was to predict the location of the expected shear failure 
crack. Depending on the different crack widths and frequencies used, different sections of the punching shear crack would appear and thus the exact location of the column was not important for this analysis, just a general location would be sufficient. By scanning the surface of the slab, the formation and location of punching shear cracks would be determined. As oppose to the following three specimens, the dynamically loaded slab would need to be analyzed using the formation of surface cracks. In order to achieve an accurate representation of the internal structure, various readings were taken of the surface. Each time a new reading was taken, the frequency of the MIRA would be adjusted higher to give more clarity of the image beginning with $40 \mathrm{kHz}$. The frequency was adjusted so that it would match the working condition of the MIRA and eliminate the error that is involved with a highly damaged specimen. The most representative four readings are shown below in Figure 3.9.

The most representative of the all the readings is shown in Figure 3.9b. The readings were taken at a frequency of $50 \mathrm{kHz}$ and show a very clear outline of the punching shear angle. Although the image is unclear for the left part of the slab, the image could be easily used to establish the angle and location of the punching shear crack for the right side of the slab. Due to the poor resolution of the left half of the slab using the $50 \mathrm{kHz}$ reading, the angle can only be estimated and assumed, and thus the other frequencies need to be used to determine its characteristics.

To estimate the angle on the left side of the slab, the reading at $60 \mathrm{kHz}$ and $70 \mathrm{kHz}$ are found to be much more representative. As the formation of cracks is not so severe in the slab, these cracks will appear only for certain wavelengths. From the $60 \mathrm{kHz}$ reading we can get an angle beside the column of $11.3^{\circ}$. From the $70 \mathrm{kHz}$ reading we can find the angle on the tensile side of the slab to be $33^{\circ}$. These two angles agree with the results found in the $40 \mathrm{kHz}, 60 \mathrm{kHz}$ and $70 \mathrm{kHz}$ readings and the left angle can be estimated to be $30^{\circ}$ on the far side from the column, and $10.65^{\circ}$ in the section adjacent to the column by averaging the results from the three readings.

The angle on the right side of the slab can be estimated using all four frequencies. The results that were obtained were relatively similar and lead to no outliers. The far angle from 
the column is estimated at $33.5^{\circ}$ and the angle adjacent to the column is estimated to be $13^{\circ}$. Both for the left and right angles, the average of the readings were used to estimate the actual angle.
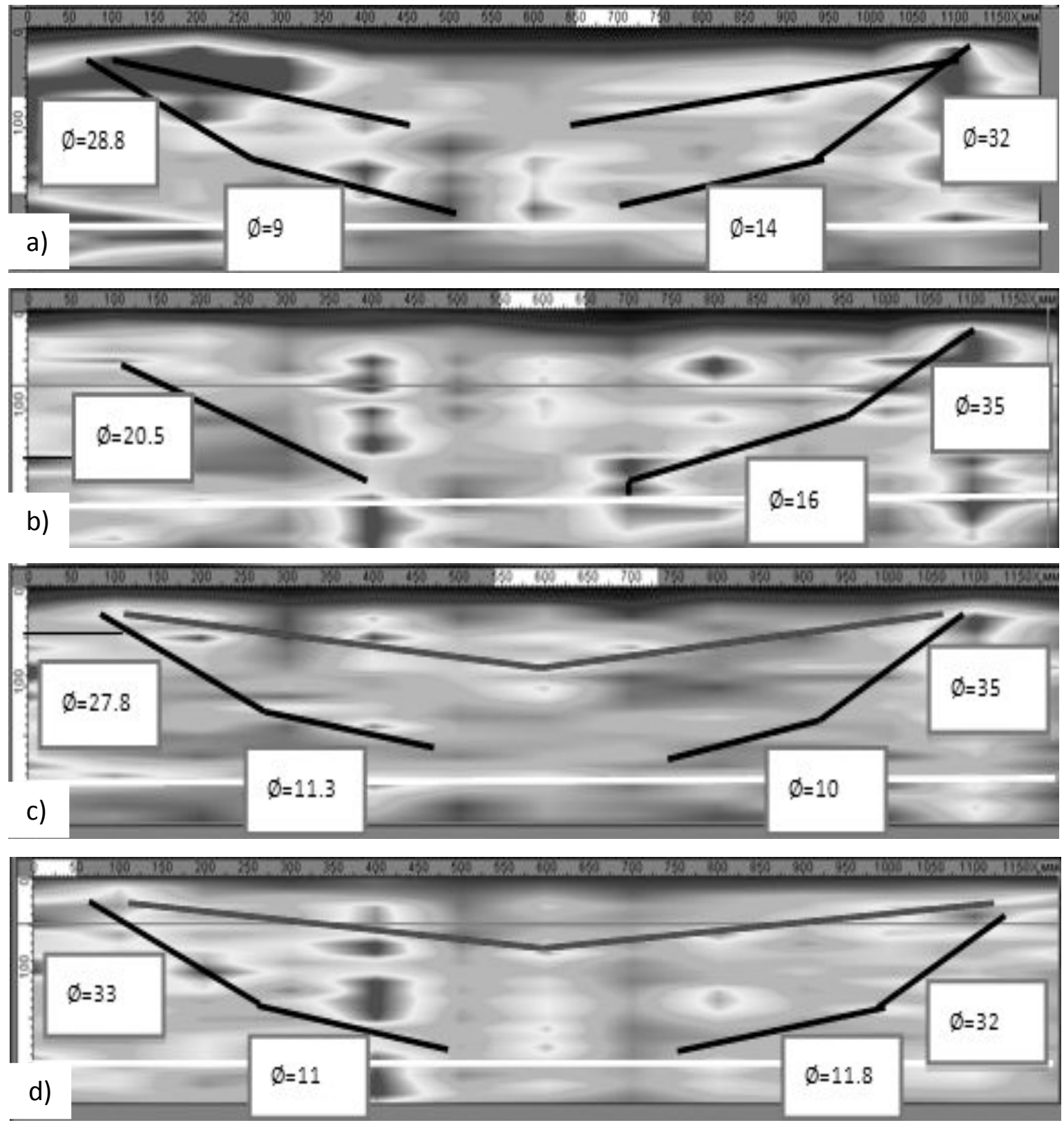

Figure 3.9: Slab 1 D-Scan Image; (a) 40 kHz; (b) 50 kHz; (c) 60 kHz; (d) 70 kHz

When the punching shear crack was traced onto the image provided by the MIRA software it was found that the punching shear crack did, in fact, lead to the surface of the test specimen, making it easier to pinpoint the location of where this crack will form. As it is 
shown in the four images, the surface cracking would appear at $1100 \mathrm{~mm}$ from the left. This location was later measured on the test surface and a surface crack was found in that location, this is the position form where the surface crack propagated into the specimen.

\subsubsection{Slab 2/HS5}

Slab 2 was tested under monotonic load up to failure. This slab is larger than the previous, and also includes T-headed shear studs. These studs would stiffen the slab around the column, and would prove to give a larger angle of punching than the slab without shear studs. Also the distance from the support to the surface opening cracking of the punching shear crack would show to occur much closer to the column than the un-stiffened slabs following.

The clearest reading for this slab came from the $60 \mathrm{kHz}$ reading in Figure $3.10 \mathrm{~d}$. The back wall reflection returning to the MIRA form the punching shear crack is visible. This is marked with the black line on the image in Figure 3.10a, and was pinpointed due to the large reflection of signal from the region surrounding it, indicating a clear air void. Although, just like in the analysis for slab 1, the thickness of the specimen was located on every image prior to doing any analysis. The thickness of the slab was marked with the white line, along with the location of the column. Once the dimension of the slab could be seen on the image, the selection of significant signal could be analyzed. This is where the $60 \mathrm{kHz}$ reading

showed, a clear image of the location of the punching shear crack. As well the reading obtained at $50 \mathrm{kHz}$ (Figure 3.10c) agree with the findings. The two readings would agree with a small crack opening that can be determined form the change in frequency and the amount of signal that is returned back to the MIRA. When the wavelength is relatively the same length as the opening of the crack, the majority of signal will not propagate through the crack and will return to the MIRA.

By adopting the concept described above, the readings in Figure 3.10a, and Figure 3.10b, should not be included in the analysis. The large wavelength was able to propagate through 
the imperfection and not provide a good measure of the angle. The results that are obtained from Figure 3.10b demonstrate similar results as Figure 3.10d.
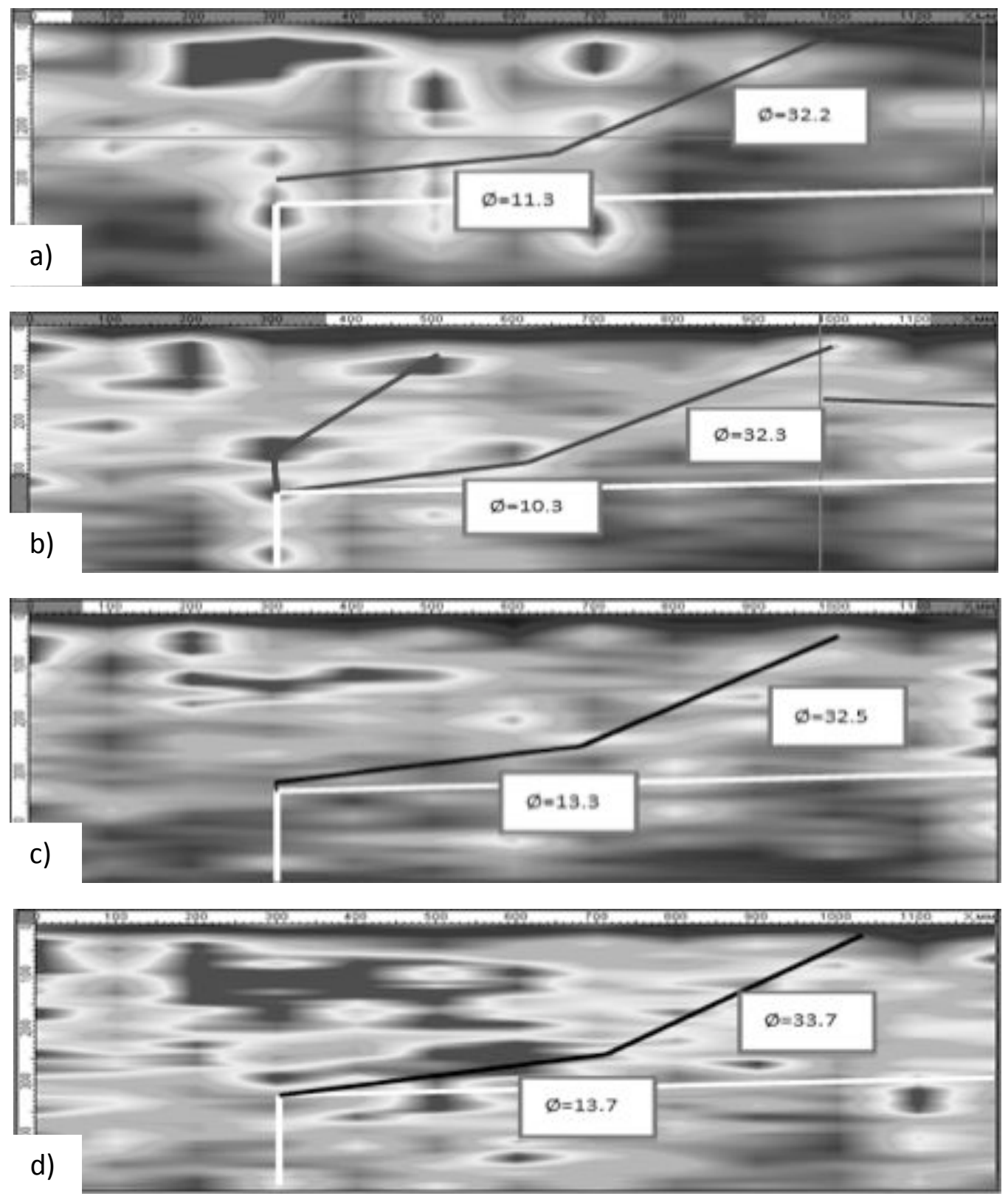

Figure 3.10: Slab 2/HS5 D-Scan Image; (a) 30 kHz; (b) 40 kHz; (c) 50 kHz; (d) 60 kHz

The location of the crack should not be considered exact, and thus only the $32.3^{\circ}$ angle can be used as an average of the readings. The reading from Figure 3.10a should be completely disregarded for this analysis. Therefore, the shear punching angle can determine to be as follows, the far angle from the column is estimated to be $33.1^{\circ}$, and the angle adjacent to 
the column is about $13.5^{\circ}$. These values were found by taking the average of 3 values for the far angle $(40 \mathrm{kHz}, 50 \mathrm{kHz}$ and $60 \mathrm{kHz})$, and 2 values for the adjacent angle (50 kHz and 60 $\mathrm{kHz})$.

\subsubsection{Slab 3/HS4}

Slab 3(HS4) is similar to slab 2(HS5), although it is $50 \mathrm{~mm}$ thicker and does not contain shear studs. The lack of shear studs appears to lead to a lot more damage to the test surface of the slab. The punching shear crack also seemed to propagate a much further distance along the slab, and could be even seen appearing at half the depth of the slab's outer most edges. Based on the MIRA results it could determine that the T-Headed Shear studs had a large impact when it came to the behavior of the punching shear cracking near the column connection.

Due to the highly damaged test surface, relatively low frequencies would have to be used. These frequencies would allow the signal to propagate despite the surface defects and allow for the analysis of the internal structure. Using such low frequencies proved to be challenging because of the low resolution of the image, and the minimal information that this provides a poor approximation of the location on the crack within the test specimen. The only reading that provided a useful result was the one taken at $50 \mathrm{kHz}$. This reading is presented in Figure 3.11.

From the $50 \mathrm{kHz}$ reading, there is only one location that the signal reflection can be considered high. The black line in the Figure 3.11 marks this region. The location of the punching shear crack was determined by connecting points of high signal reflecting starting with a point at the outer most edge of the slab, and the other near the location of the column connection. These two lines ended up converging to one point, and showed two different angles just as slab 1 and 2. Once the location of the crack was determined the location of the crack at the far right of the image was kept constant, as it was the only point that was part of the punching shear crack. 
The angle of punching shear for the slab was consistent to that of the results obtained form the first two slabs, as there are two angles within the slab. The far angle from the column can be estimated by the MIRA readings to be $26^{\circ}$, and the near angle to be $6.7^{\circ}$. The result would show that the shear studs greatly stiffen the slab as the angle near column is nearly doubled with the shear studs.

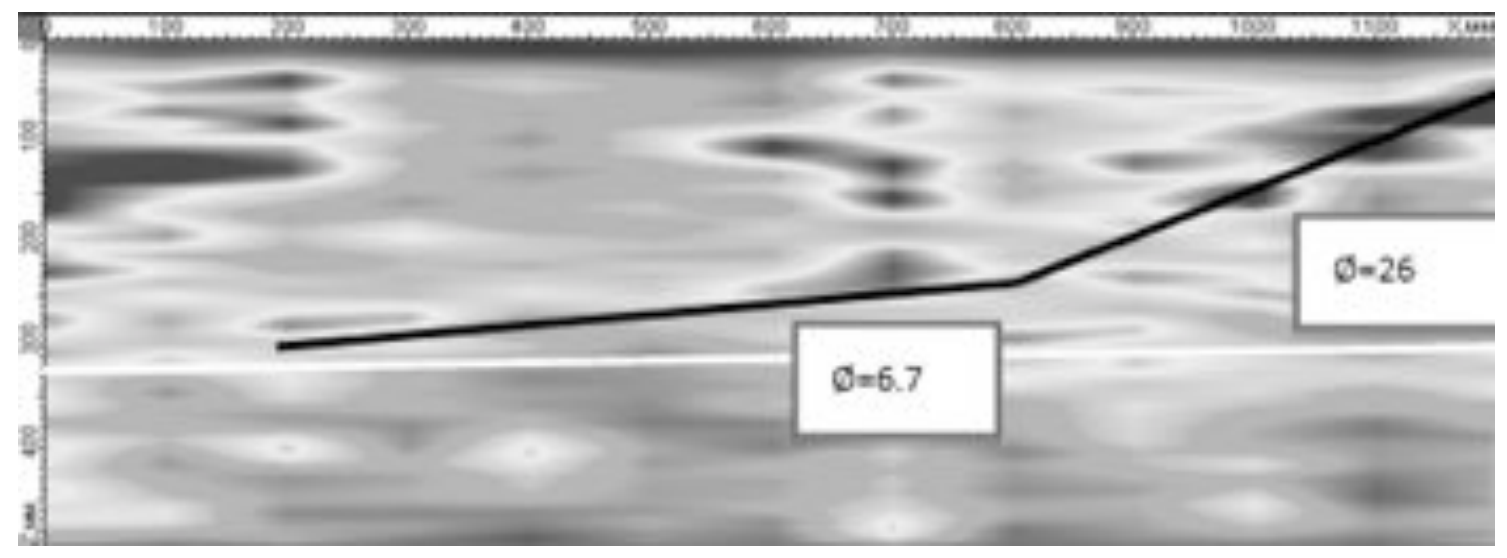

Figure 3.11: Slab 3/HS4 D-Scan Image 50 kHz

\subsubsection{Slab 4/HS7}

Slab 4(HS7) was part of the same experimental program as Slab 3(HS4). The slab does not contain shear studs, and the stiffness should be similar to that of HS4. The slabs overall depth is $400 \mathrm{~mm}$ and the angle of the punching shear crack would slightly differ from that of Slab 3.

The results obtained from the MIRA, in this case, were difficult to interpret due to a high internal damage within the slab. Various readings were taken using a wide range of frequencies, although the majority returned no significant information on the location of shear punching crack. Only the two readings, $40 \mathrm{kHz}$ and $60 \mathrm{kHz}$, gave a slight indication of the location of the shear cracking.

Figure 3.12a gives a good indication of the extent of damage that can be found within the slab. Multiple reflections appearing in the first $200 \mathrm{~mm}$ of the slab represent the tension cracking. The punching shear crack can be determined by the amount of signal that reaches a certain depth within the slab. Unlike the last 2 slabs the back-wall reflection cannot be 
used to pinpoint the location of the crack. Due to the high level of damage in the first 200 $\mathrm{mm}$ of the slab a large amount of signal is reflected back and very minimal signal reaches the punching shear crack. As well, any signal that will reach the shear crack will be reflected back immediately. Thus any reflection that would appear at a distance much grater than $200 \mathrm{~mm}$ within the slab would be a reflection coming form the shear crack.
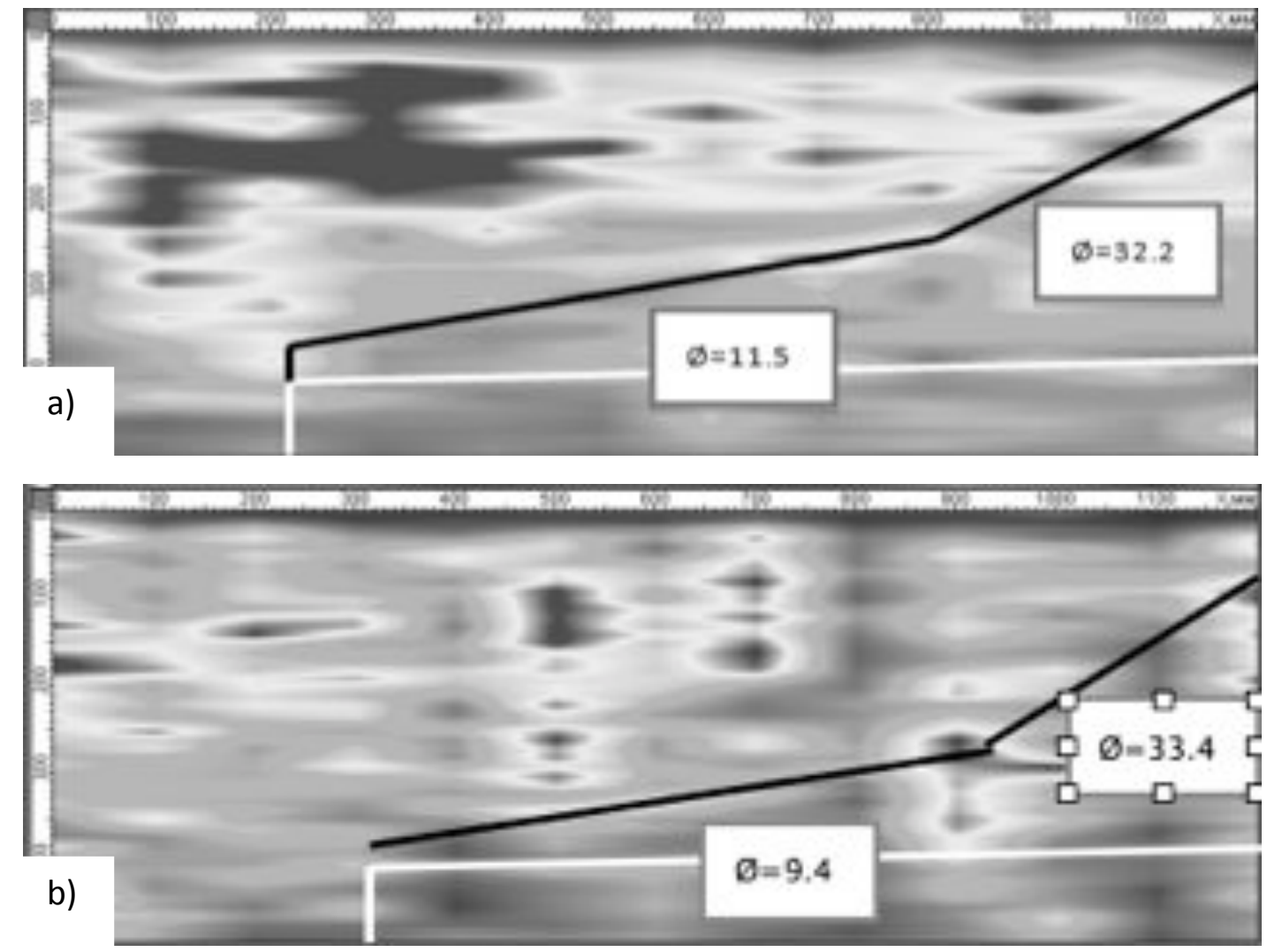

Figure 3.12: Slab 4/HS7 D-Scan Image; (a) 40 kHz; (b) $60 \mathrm{kHz}$

In Figure 3.12a this effect can be seen very clearly with the reflection $300 \mathrm{~mm}$ deep and 700 $\mathrm{mm}$ along the reading surface. This is a clear sign of an imperfection within the slab. Also there is a reflection near the column that would indicate the separation of the slab and the column on the opposite side of the slab. The first angle, near the column, can be estimated by connecting two points of high reflection. Upon doing so it becomes more evident that the signal underneath this line in Figure 3.12a appears to be much weaker as oppose to the one on top of the line, again suggesting a separation within a slab. 
The second angle, on the far end of the slab, was determined by looking at Figure 3.12b. Here there is a reflection at the right most limit of the image that would suggest a strong reflection of signal near this point. The location of the shear crack was plotted in Figure $3.12 \mathrm{~b}$ and the lines were connected with the region of damage $700 \mathrm{~mm}$ along the test surface. This would give the second angle.

In order to verify these results Figure $3.12 \mathrm{~b}$ was used the lines in similar locations and notice if there is reflection that would overlap with the lines. These were found in the same points of the image, and the same reduction in signal beyond the estimated location of the punching shear crack can be noticed. The estimated angles for this slab were $10.5^{\circ}$ for the angle beside the column, and $32.8^{\circ}$ for the far angle.

\subsection{Evaluation of MIRA's Capabilities}

The MIRA tomograph proves to be an effective tool for the non-destructive evaluation various structural components. From determining the grouting condition of prestressed ducts within bridge girders, to determining the mode of failure of concrete slabs, the MIRA tomograph can provided enough information in order to assess the internal structure of the concrete member.

Within this report it was clear that shear wave tomography could be used for determining the punching shear angles within thick two-way slabs. In some cases it proved to be more effective than others, although this is largely due to the severe damage that the slabs have been exposed to. The MIRA is meant for structural health monitoring of building structures, yet it was effective in determining the angles and location of shear crack within the slab. The results that the MIRA gave were not straightforward and thus an analysis of the results, using the various operating frequencies needed to be carried out for proper interpretation.

The analysis showed that there are in fact 2 angles that represent the punching shear failure at the ultimate load. One angle of low grade can be seen, near the column that applied load, and an angle that is much greater at a distance away from the support that leads to a surface opening crack. These angles can change with the change in depth and size of the 
slab, and are largely affected by the introduction of shear studs to enhance the ultimate capacity of the slabs.

Shear wave tomography also proved to be effective in determining the internal structure of multiple concrete elements. It can easily provide information on the location of reinforcement, voids and cracking within concrete structures up to $1.0 \mathrm{~m}$ in thickness with moderate reinforcement ratios. When it comes to high reinforcement ratios as seen in the structural floor, the signal resolution is greatly attenuated by the many obstructions found within the test specimen and can render the results insignificant. Although the machine has a specified capability of reading signals to a depth of $2500 \mathrm{~mm}$, it may not be the case for concrete structures. Material inconsistencies such as gradation and reinforcement found the capability the MIRA tomograph to only about $1600 \mathrm{~mm}$ after which the signal is completely attenuated and there is very minimal reflections returned to the antenna leading to inaccurate and irrelevant reading of the internal structure. 



\section{Chapter 4 | \\ Proposed \\ Piezoceramic System}

The following section will present the methodology behind the proposed active nondestructive health monitoring system. The section will begin by describing the concept of active non-destructive evaluation, and present the operating principle of the developed system. The section will then present the various components that comprise the system, starting with the development of the embedded piezoceramic sensors, and following with a brief description of the equipment required to complete a reading using them. The section follows with an introduction into the signal processing methodology, including a sample of the raw data obtained from the digital oscilloscope. 


\subsection{Concept}

The concept of active non-destructive evaluation of reinforced concrete structures is a concept that has been researched for many years. This methodology was first discovered in the literature in a paper by Wu et. al. (2006) and is illustrated in Figure 4.1.

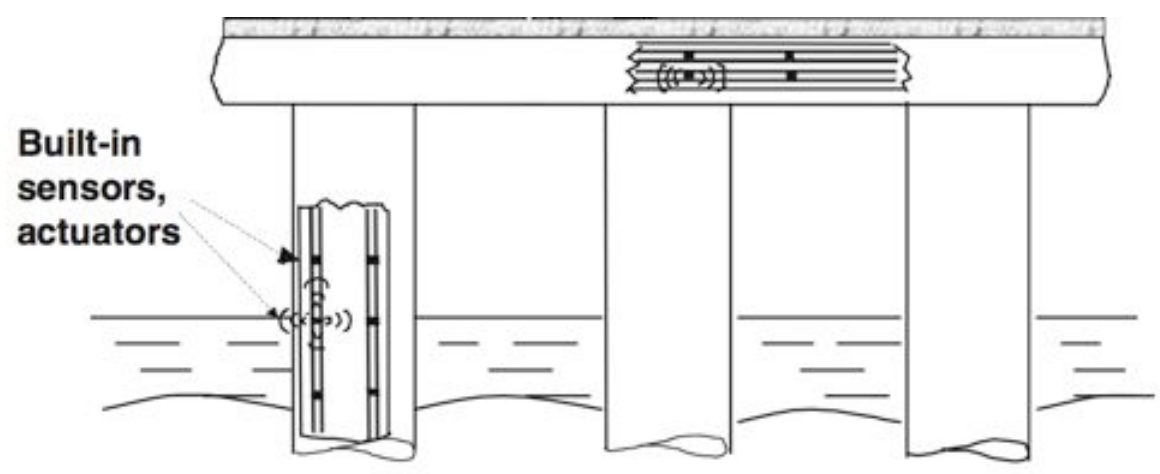

Figure 4.1: Active Non-Destructive Health Monitoring Concept (Wu and Chang, 2006)

The theoretical system would utilize embedded piezoceramic transducers to determine the delamination of reinforcement within a concrete structure. Multiple sensors would be installed in the entirety of a reinforced concrete structure in a predetermined array. The sensors could then be utilized to determine the appearance of damage between a specific signal path by introducing a mechanical stress into that medium, and analyzing the received component. By automating this process, the system could be used to constantly monitor the condition of a reinforced concrete structure. This system would provide the structural inspector with continuous real time information about the condition of the desired structure. An active system could provide location based warning of member conditions and will facilitate the overall maintenance process, and prioritizing the rehabilitation process of structures. By allowing maintenance crews to quickly assess the structural condition of the structure under investigation, the system would provide a means of organizing the work of maintenance workers to a priority-based assessment of the structure.

The location of the piezo-sensors in the predetermined array could easily be adapted to provide location-based information on the internal condition of the concrete member. The concrete member can be divided into the desired sub regions as demonstrated in 
Figure 4.2. The denser the mesh of transducers in a member, the more accurate the location information provided by the sensor array is. As the signal paths between piezosensors are one dimensional, that provides information along a single line of travel, only the intersection of a crack with the signal path would be detected. The larger the distance between two adjacent transducers is, the more likely it is for a signal path to be intercepted by a crack, and thus the less accurate the representation of the cracks location. Having a denser mesh reduces the probability of a crack to intersect a signal path, thus giving a higher degree of accuracy for the cracks location within the concrete member. The installation of the piezo-sensors within the matrix of a reinforced concrete slab has the ability of determining the profile of a longitudinal crack initiating across the slab.
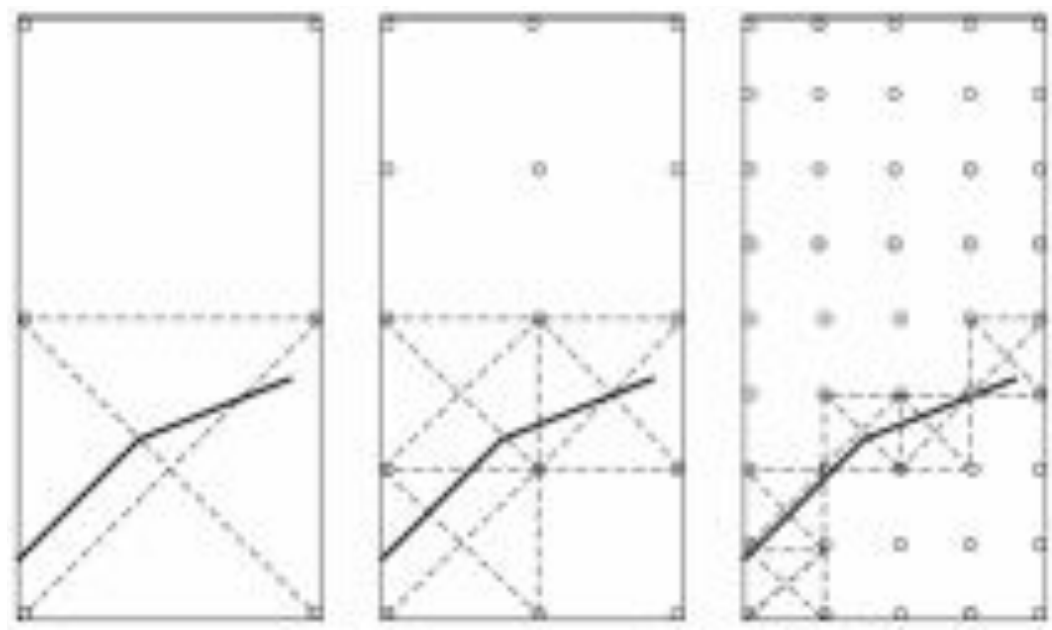

Figure 4.2: Density of Transducer Mesh (Sun and Yan, 2008)

The proposed piezoceramic sensor system works on the principles of harmonic guided waves. These harmonic stress waves are generated at an arbitrary position within the concrete member, and transmitted through the concrete matrix before being received by a piezoceramic patch. Since the stress waves are induced by one piezoceramic patch (actuator), and received by another piezoceramic patch (receiver), the information gathered by each pair would be representative of the straight-line portion between the two sensors. The initiation of a crack through a signal path would cause a break in the transmissibility, reducing the signal's strength. 
Concrete was found to have an attenuation constant of $2.8 \mathrm{~dB} / \mathrm{cm}$ at its natural frequency by Rao et al. (2003). This means that if it's not strong enough, the energy of any stress wave can be quickly dissipated within the concrete matrix, and become useless for analysis. Piezoceramic patches are very useful, in this regard, as they have the ability to induce stress waves at very high amplitudes and help eliminate the problems associated with this high attenuation constant. Although, the same property that impedes the energy transmission of stress waves in concrete can also help eliminate any noise that may be experienced in the transmission of guided waves. The introduction of a guided wave in a medium does not necessarily follow a linear path, but rather travels spherically through a medium. When a wave comes in contact with an interface between two materials having a large difference in impedance, or the surface of a concrete member, multiple reflections are created classified as noise. At an attenuation of $2.8 \mathrm{~dB} / \mathrm{cm}$ the signal's power is halved for every centimeter it travels through the medium. Thus the signal that is being reflected off the concrete-air interfaces is shadowed by the direct path signal between the two piezo-transducers, and becomes negligible.

\subsection{Sensors}

Piezoceramic materials are very brittle in nature making them very fragile and delicate to handle. The introduction of these electronic components to the harsh concrete environment would damage the piezoceramic material, and render the sensors useless. Fixing the sensor inside the formwork prior to the beam being cast is also a key concern. The systems' ability to detect and quantify damage induced in a member is only practical if the operator can identify the location where the damage is, thus the location of the piezosensors in very important. For the system to function the sensors should be installed in such a way that they exhibit a very rigid behavior during the casting process, withstanding the high stresses and strains exhibited, and remain in the desired location after the casting process is complete.

Electronics have very little tolerances for moist or wet environment, and piezoceramics are no different. The exposure of these sensors to the residual moisture of concrete would 
short-circuit the sensors, voiding their ability to convert electrical signals to mechanical stress waves. In order to address this issue, the sensors require a watertight casing to create a barrier between the residual water inside the concrete, and the piezoceramic patch. This casing has to provide a very efficient energy transfer between the piezoceramic patch and the concrete, as to not attenuate the induced mechanical wave, but rather facilitate its transfer into the concrete environment. In order to achieve a good transfer of mechanical energy between the three materials, (piezoceramic, casing, concrete) the casing material should facilitate the transfer of mechanical energy between the piezoceramic patch and the concrete medium.

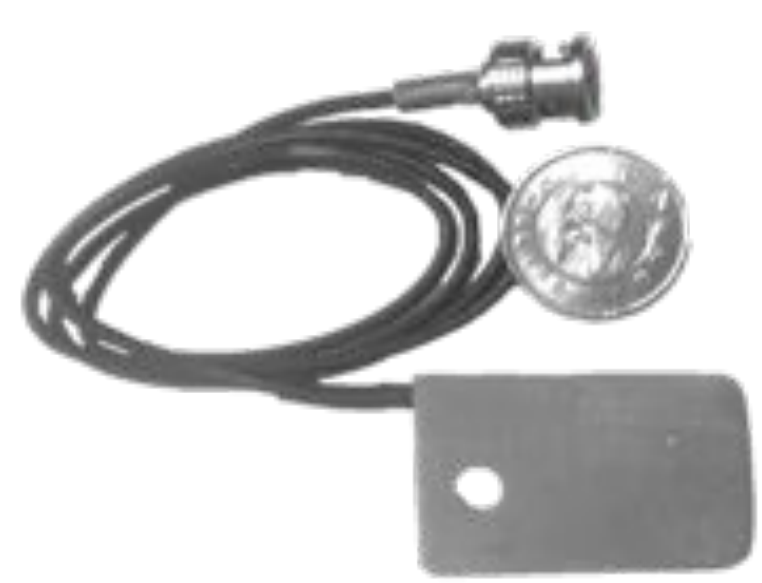

a)

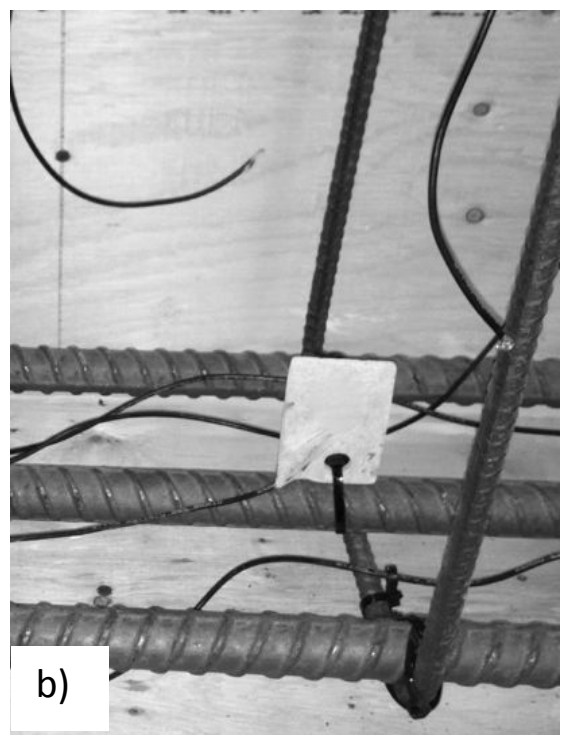

Figure 4.3: Piezoceramic Sensor

The final piezoceramic sensors utilized in the proposed non-destructive system is presented in Figure 4.3a. The sensors contained a square piezoceramic patch having a dimension of 12 $\mathrm{mm} \times 12 \mathrm{~mm} \times 0.3 \mathrm{~mm}$, cast into a high strength epoxy casing measuring $30 \mathrm{~mm} \times 50 \mathrm{~mm} \times 2$ $\mathrm{mm}$. The piezo-patch was wire connected to the external data acquisition system using coaxial cabling and a BNC connector. The sensors would be attached directly to the steel reinforcement bars using a pair of zip-ties as illustrated by Figure 4.3b. The strong epoxy casing ensured that the fragile piezoceramic patch is not damaged during the concrete casting process, as well as seal the piezoceramic patch from the residual moisture in the concrete. 


\subsection{Equipment}

The proposed system uses three distinct steps to complete a reading detailed in Figure 4.4. The desired sweep frequency is generated using an auxiliary function generator, and passed through a custom-made high output power amplifier before it's transmitted to a piezosensor and translated to a mechanical wave. The stress wave passes through the concrete matrix generating a frequency spectrum representative of the vibration-characteristic of the section. This spectrum is received by a secondary piezo-sensor and is translated back to an electrical signal, which is amplified using a low power amplifier, and sent to a digital oscilloscope where it can be processed to determine the desired Health Monitoring Index (HMI) for that specific section.

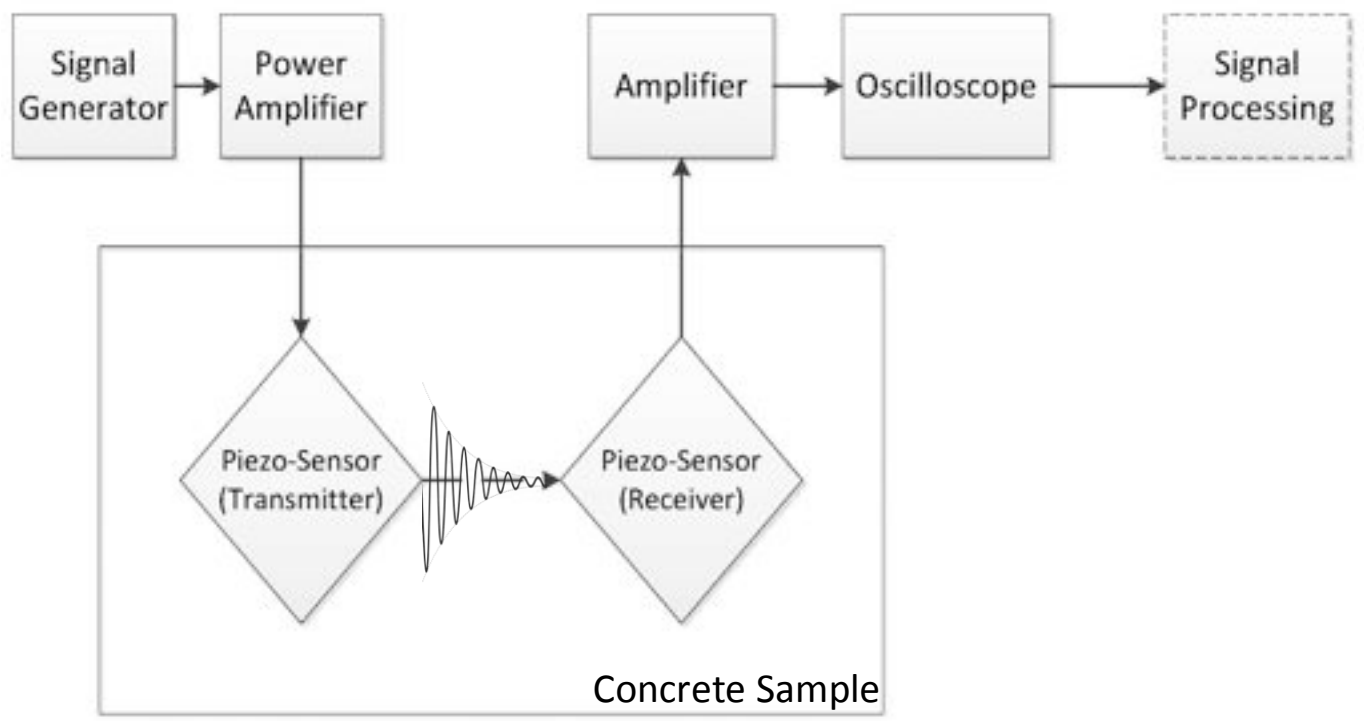

Figure 4.4: Signal Flow Chart

\subsection{Signal Processing for Health Monitoring}

As concrete is not an isotropic material, yet is comprised of a complex matrix of granular materials, there is not one specific ultrasonic frequency that would dominate the material's excitation. In order to effectively determine the materials transmissibility, and be able to evaluate the extent of damage on a signal path between two sensors, a wide band of frequencies needs to be implemented. For this reason a sweep frequency was employed. Each frequency will attenuate at a different rate as it travels through the concrete medium 
and produce the vibration signature of the specific region between two sensors. Figure 4.5 demonstrates a sample output signal acquired from the receiving piezo-sensor after it was processed using the Fast Fourier Transform into the Amplitude-Frequency Spectrum.

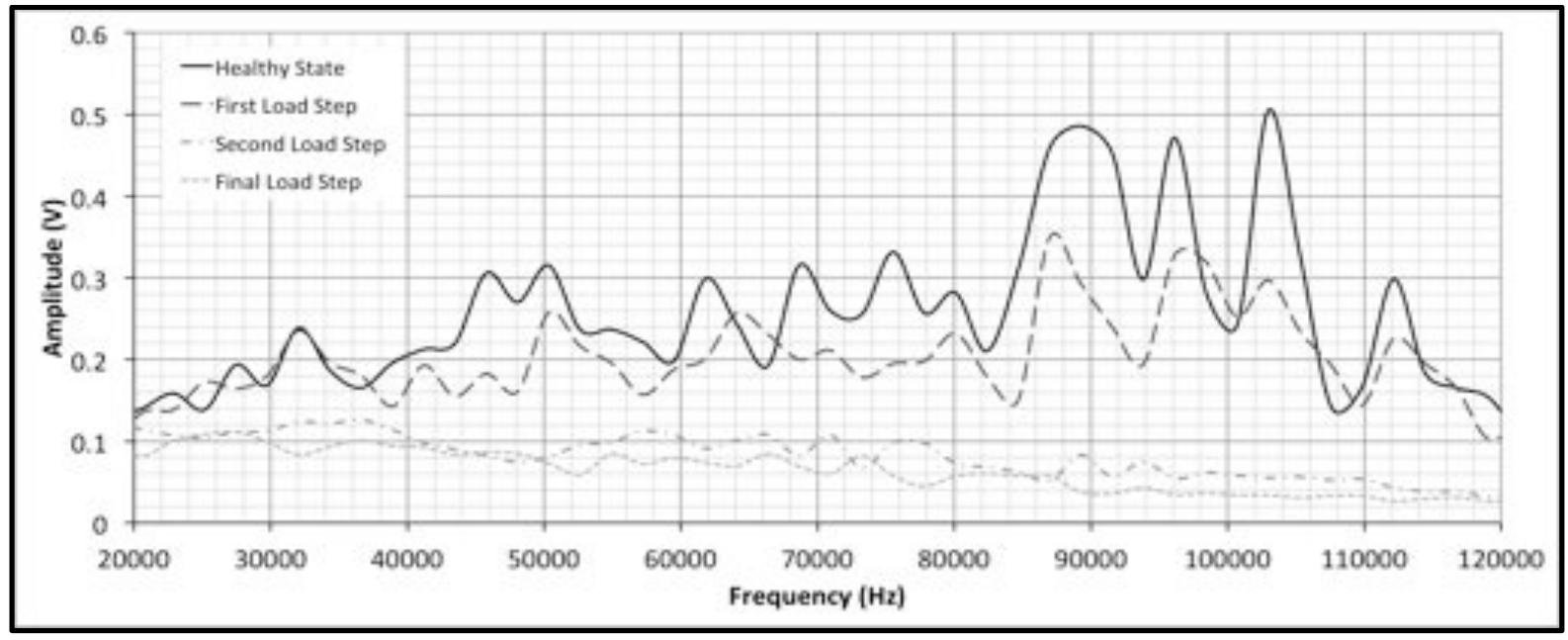

Figure 4.5: Sample Output Data (Health Monitoring)

As the load in the beam is increased the signal's strength is seen to decrease. This decrease in signal strength is due to the introduction of strain between the two sensors separating them further and further apart. The concrete region presented in Figure 4.5 cracked at the third load step. The appearance of cracking attenuates the majority of the signal's strength for frequencies exceeding $70 \mathrm{kHz}$. The attenuation of the frequency spectrum is the primary focus of the signal processing used by the piezoceramic system. The wave's energy is converted to the Root Mean Square (RMS) and compared to the original, healthy state wave. The RMS values are a very useful measure of a wave's average amplitude during the duration of its lifetime, thus a tool for comparing the overall power of a wave.

The amplitudes of the received waves were recorded and analyzed using Equation 4.1. The equation is a normalization of the commonly accepted Root Mean Square Deviation (RMSD) equation used for spectral analysis, and has been previously adopted for concrete research by Sun and Yan (2008) The Health Monitoring Index (HMI) is a function of the difference between the amplitude of frequency ' $n$ ' prior to the load being applied $\left(A_{f n, 0}\right)$, and the amplitude of frequency ' $n$ ' at a given time during the members lifetime $\left(A_{f n, t}\right)$. The results 
obtained by the $\mathrm{HMI}$ are indicative of the sections vibration-characteristic, or transmissibility, and thus representative of the section's condition.

$$
\mathrm{HMI}=\sum_{n=0}^{\infty} \sqrt{\frac{\left(A_{f n, 0}-A_{f n, t}\right)^{2}}{\left(A_{f n, 0}\right)^{2}}}
$$

\subsection{Signal Processing for Concrete Early Strength Prediction}

A wide variety of research has been done to determine the applicability of piezo-ceramics to the development of concrete's early strength, including the determination of concrete's early strength based on wave propagation principals, and amplitude analysis. Although both methods provide reasonable results, the analysis of the signal can be rather difficult, and/ or impractical. The method based on wave propagation principals requires the investigation of two types of stress waves, and relating the results using the fundamental shear and pressure wave equations to determine the Modulus of Elasticity, and Poisson's ratio of the concrete sample. Although, this analysis has demonstrated very promising results, and provides a direct correlation between the concrete's condition and stress wave analysis, the signal processing and equipment necessary to complete a reading is expensive, complicated, and impractical for internal condition assessment when a member's surface is inaccessible.

The amplitude based method proposed by Song et al. (2008) also showed a good means of predicting the development of concrete's early strength. The signal processing was conducted utilizing a Fuzzy correlation, which would rank the concrete's strength based on a set of predetermined conditions. The results of the analysis were shown to have a good correlation with the results obtained from the destructive standard cylinder method. However for the purpose of developing a system that can both determine a member's internal condition, and the development of concrete's early strength, the signal processing methods need to differ from one another as to be differentiable by computer software. The 
health monitoring principal for the proposed piezo-ceramic system in this investigation, relies on the change in amplitude of an induced frequency spectrum, Thus the analysis carried out for the development of concrete's early strength should rely on a different principal, so that a computer software could differentiate between the two mechanisms.

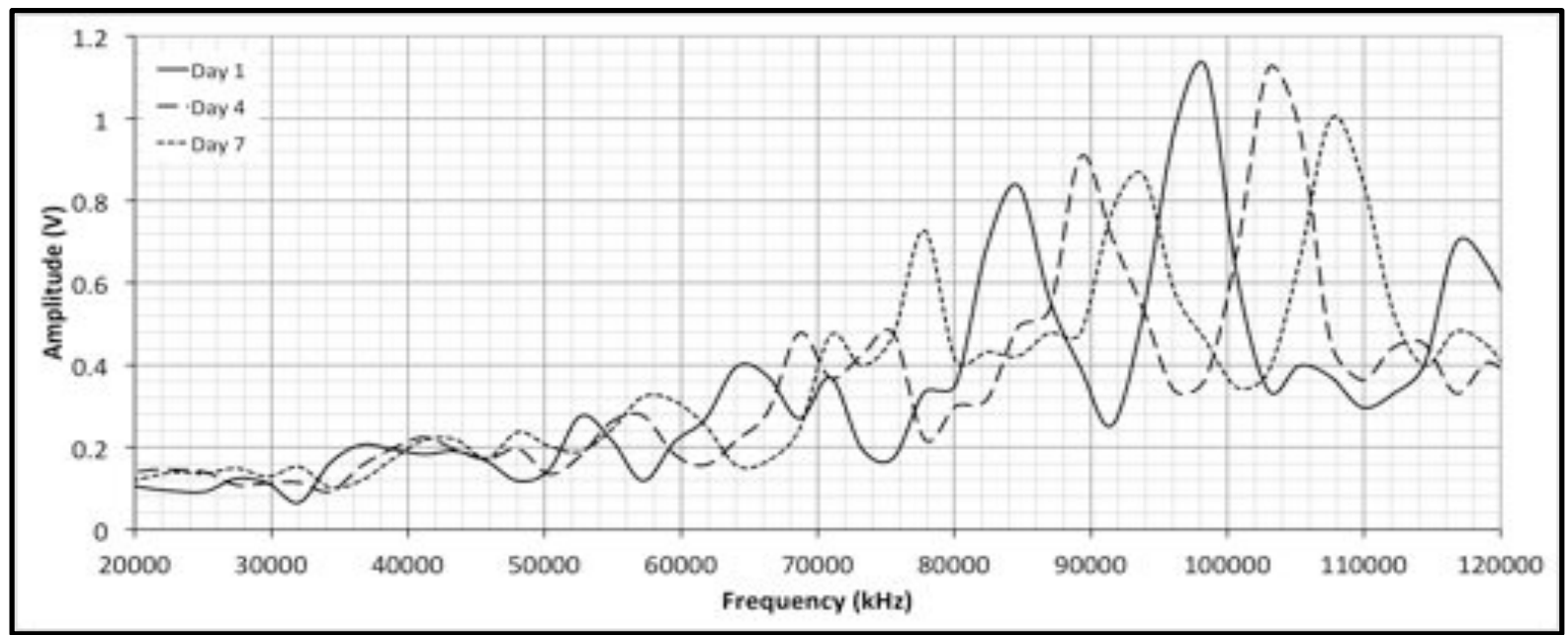

Figure 4.6: Sample Output Data (Early Strength)

Figure 4.6 illustrates a sample frequency domain of a sample that was obtained at three intervals during the samples curing period. The data illustrates a significant shift in the frequency response spectrum, or vibration characteristic of the sample. As the concrete cures, and gains strength, the peak amplitudes shift to higher frequencies. This shift in natural frequencies, as well as frequencies exhibiting the highest attenuation of signal, is directly proportional to the materials gain in rigidity, or concrete strength. The analysis for the development of early strength of a concrete member will be carried out on the smallscale samples, as to develop a relationship between the results obtained from the compressive cylinder's strengths and the shift in frequency response period. The shift will be investigated at various locations of the amplitude-frequency curve for each sample tested, and the vibration characteristic will be read in 24-hour intervals. The shift in the vibration characteristic graph will be compared directly to the vibration characteristic graph of the obtained in the first 24-Hours after casting. 



\section{Chapter 5 |}

\section{Experimental Program}

The following experimental program was carried out to determine the effectiveness of embedded piezoceramic-sensors for lifetime heath monitoring of reinforced concrete members. The program included testing nine reinforced concrete beams designed and constructed in accordance to CSA-A.23. Seven beams were used as part of a small-scale investigation, and two were part of a full-scale program.

The small-scale test specimens were equipped with crack-gauges and the proposed piezosensors. These beams were used to investigate the piezo-sensors' effectiveness for predictive health monitoring, and to establish a relationship between the sensor signals, and concrete strain/crack width. The beams were also used in determining the piezosensors' ability to monitor the development of concrete's early-strength. 
The full-scale beams were designed to verify the effectiveness of the piezo-sensors as part of a complete health monitoring system. The two beams were equipped with FBG fiber optic sensors for localized strain readings, as well as the proposed piezo-ceramic sensors. The beams were also scanned using the MIRA 3D tomographer, to establish the effectiveness of the piezo-sensors in comparison to a commercially available nondestructive test system, and verify the validity of the investigation carried out in the small scale experimental series.

Throughout the experimental investigation presented here all members were designed, and built, using high strength concrete of $50 \mathrm{MPa}$ at 28-days, nominal aggregate size of $14 \mathrm{~mm}$, and slump exceeding $150 \mathrm{~mm}$. Steel reinforcement bars having a yielding strength of 450 MPa were used for both the shear and flexural reinforcement. All shear reinforcement (stirrups) was fabricated using $10 \mathrm{M}$ rebar.

\subsection{Specimen Identification}

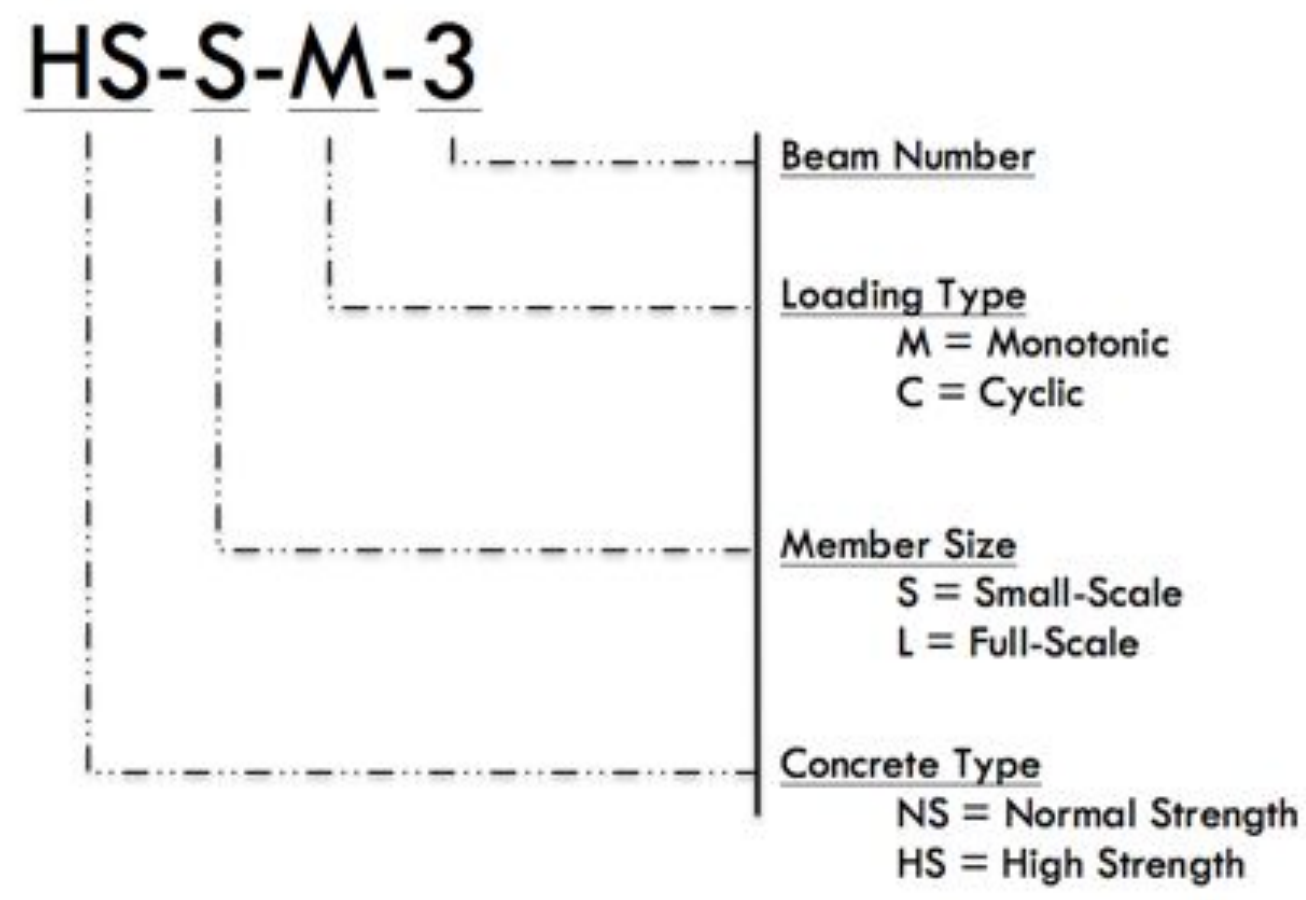

Figure 5.1: Beam Identification Scheme 
Table 1: Reinforced Concrete Beams of the Experimental Program

\begin{tabular}{|c|c|c|c|c|c|c|}
\hline Beam & $\begin{array}{l}\text { Cross- } \\
\text { Section }\end{array}$ & Length & Load Setup & $\begin{array}{l}\text { Loading } \\
\text { Type }\end{array}$ & $A_{s}$ & $\begin{array}{l}\text { Stirrup } \\
\text { Spacing }\end{array}$ \\
\hline $\begin{array}{l}\text { HS-S-M-1 } \\
\text { HS-S-M-2 } \\
\text { HS-S-M-3 } \\
\text { HS-S-M-4 }\end{array}$ & \multirow{2}{*}{$\begin{array}{c}200 \mathrm{~mm} \mathrm{X} \\
300 \mathrm{~mm}\end{array}$} & \multirow{2}{*}{$1000 \mathrm{~mm}$} & \multirow{2}{*}{$\begin{array}{c}\text { Three-Point } \\
\text { Load }\end{array}$} & Monotonic & \multirow[t]{2}{*}{$300 \mathrm{~mm}^{2}$} & \multirow{2}{*}{$125 \mathrm{~mm}$} \\
\hline $\begin{array}{l}\text { HS-S-C-1 } \\
\text { HS-S-C-2 } \\
\text { HS-S-C-3 }\end{array}$ & & & & Cyclic & & \\
\hline$\frac{\text { HS-L-M-1 }}{\text { HS-L-M-2 }}$ & $\begin{array}{c}350 \mathrm{~mm} \mathrm{X} \\
700 \mathrm{~mm}\end{array}$ & $4000 \mathrm{~mm}$ & $\begin{array}{l}\text { Four-Point } \\
\text { Load }\end{array}$ & Monotonic & $1500 \mathrm{~mm}^{2}$ & $\begin{array}{l}500 \mathrm{~mm} \\
200 \mathrm{~mm}\end{array}$ \\
\hline
\end{tabular}

The current investigation presents a total of nine experimental samples. The experimental program comprised of both small-scale and full-scale experiments, as well as cyclic and monotonic loading programs. Figure 5.1 demonstrates the identification scheme by which the test samples can be distinguished. The first index represents the type of concrete used to cast the sample, the second index represents the size of the member, the third index represents the type of loading routine, and the fourth index represents the specimen number. As tests were repeated for the small-scale samples so that consistent results could be compared, a maximum of four samples for any test are presented. Table 1 summarizes the nine beams tested in this investigation, along with their design configuration and loading program.

\subsection{Small Scale (HS-S-M \& HS-S-C)}

The scope of the small-scale experimental program was to determine the piezo-sensors' ability to predict, detect, and quantify the appearance of a flexural crack in a reinforced concrete member. The seven reinforced concrete beams were designed in such a way that a single flexural crack can be initiated, and controlled at the mid-span of the member. Form 
the results obtained in this investigation a model was developed to relate the piezo-sensors' signals to the effective strains and crack width a member might undergo in a monitored region. The early strength development of the samples in the first seven days after casting was also monitored using the piezo-sensors, and the method described in Section 4.5.

\subsubsection{Test Specimen}

The seven beams were designed to resist an ultimate bending moment of $52 \mathrm{kN} \cdot \mathrm{m}, \mathrm{a}$ cracking moment of $16 \mathrm{kN} \cdot \mathrm{m}$, and exceed a shear capacity of $150 \mathrm{kN}$; thus ensuring flexural failure. The beams had a height of $300 \mathrm{~mm}$, base width of $200 \mathrm{~mm}$, and unsupported span of $800 \mathrm{~mm}$. The design requirements dictated that each beam would have three $10 \mathrm{M}$ bars to act in the tension zone, two $10 \mathrm{M}$ bars in the compression zone, and stirrup spaced at 125 $\mathrm{mm}$, as illustrated by Figure 5.2.

All seven beams were loaded in $5 \mathrm{kN}$ load steps, and readings were recorded at every load step using the piezo-sensors, and crack gauges. Along with the reading obtained from the equipment, the cracking pattern of the beam was visually observed throughout the duration of the experiment.

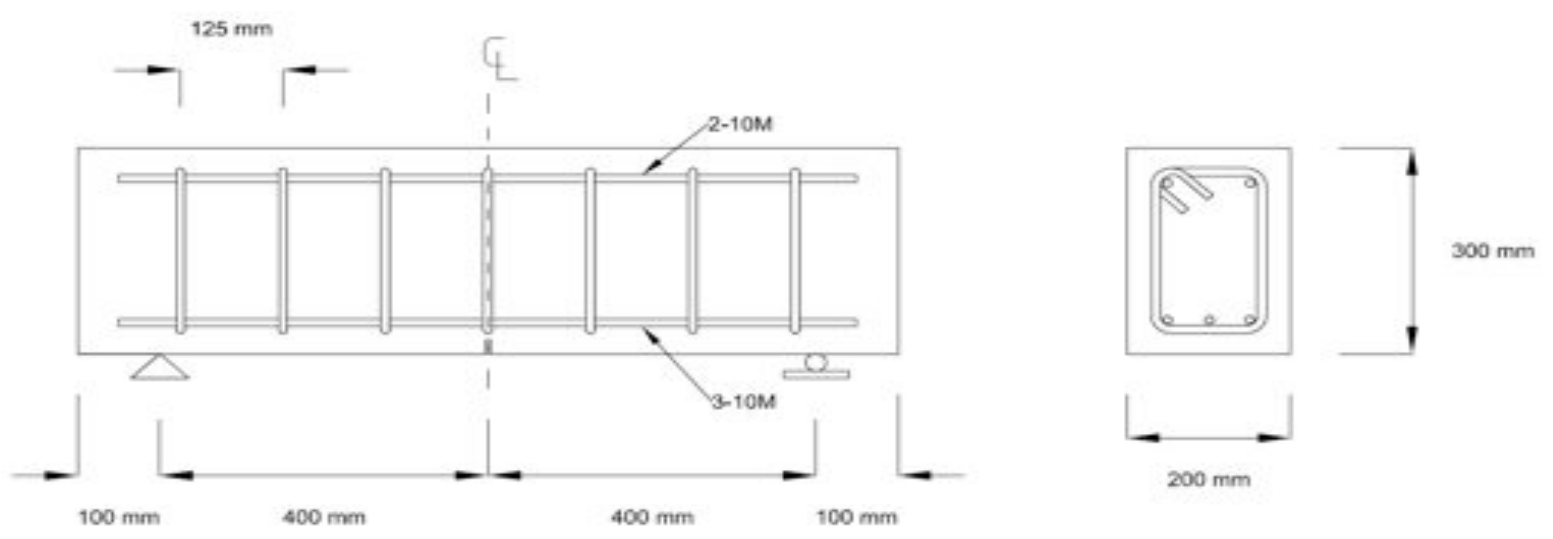

Figure 5.2: Small Scale Beams' Reinforcement Detail

\subsubsection{Small-Scale Member Fabrication}

The following sections details the specimen fabrication process undertaken for the smallscale experimental program. The details for the construction of the formwork, placement of 
reinforcement, casting, and curing processes are presented herein. Ready mix concrete was used in casting of the specimens having a minimum 28-day strength requirement of 50 $\mathrm{MPa}$, and a slump exceeding $150 \mathrm{~mm}\left(6^{\prime \prime}\right)$.

\subsubsection{Formwork}

The formwork used in the casting of the small-scale beams was made out of timber, and is illustrated in Figure 5.3. The wooden molds consisted of five panels assembled together using nuts and bolts. The beams' sides were made from 5-ply plywood of 3/8" thickness, reinforced with stiffeners made from timber with a cross-section of $2 "$ x 4". The vertical and lateral stiffeners would ensure that no distortion in the member's dimensions would occur during the casting process. The joints between each beams' side were water sealed using silicone, as to keep the moisture of the concrete confined inside the mold.

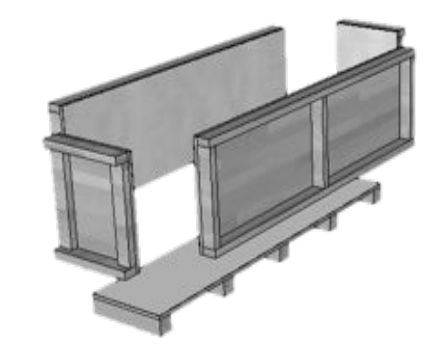

a)

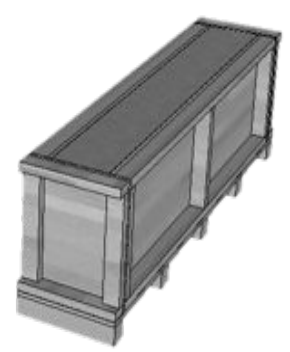

b)

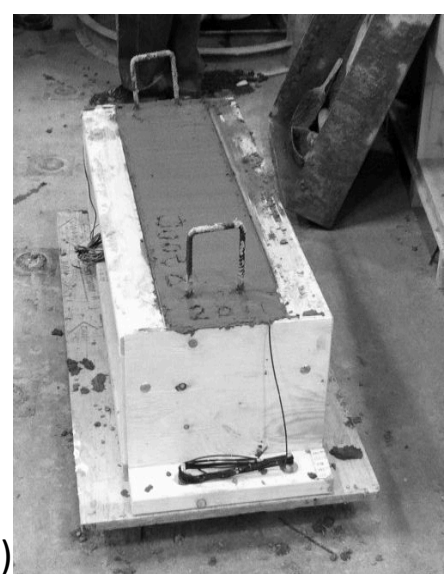

Figure 5.3: Formwork; (a) Assembly; (b) After Casting

\subsubsection{Reinforcement}

The steel reinforcement used in the assembly of the reinforcement cage had yield strength of $450 \mathrm{MPa}$. A total of seven prefabricated stirrups comprised the reinforcement cage of each small-scale member. The stirrups were attached directly to the flexural reinforcement using zip-ties. The three tensile flexural bars in the specimen were attached to plastic seats as to provide the $50 \mathrm{~mm}$ clearance with the bottom of the mold and create the clear 
concrete cover required. Two hangers were also fabricated and implemented as part of the reinforcement cage. The hangers were placed one at either end of the beam, as to provide a means of transporting the member once it is cast and ready to test. The placement of the reinforcement cage in the wooden mold is illustrated in Figure 5.4.

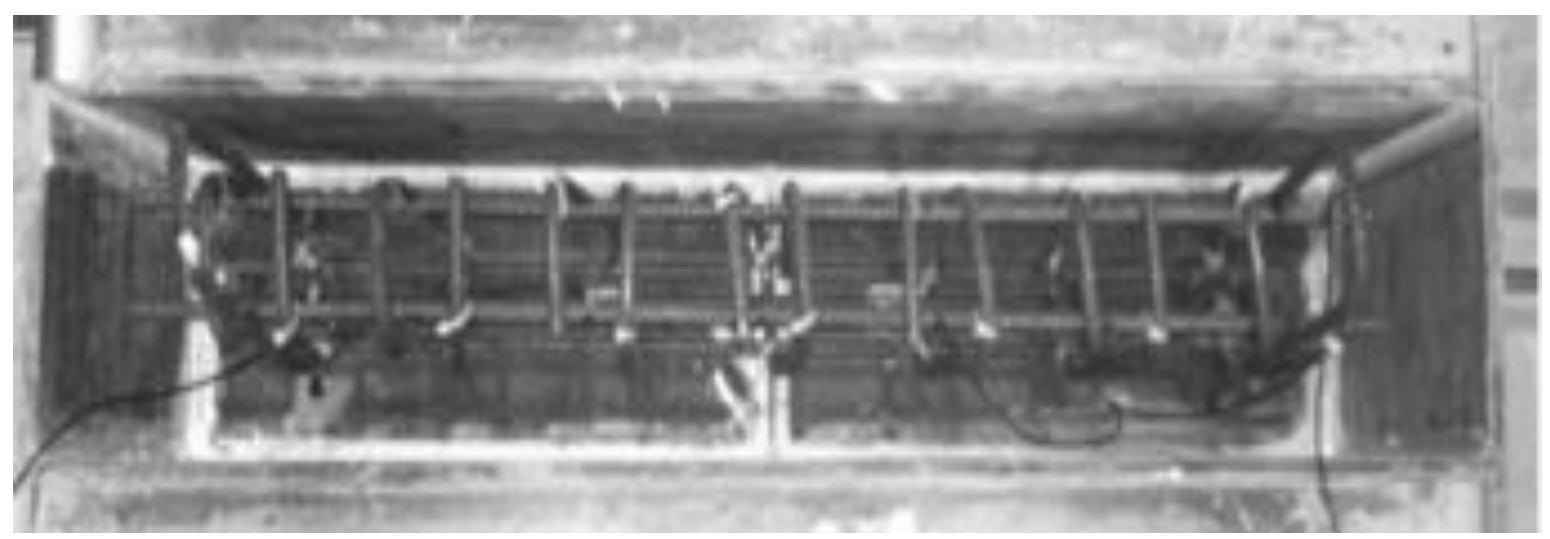

Figure 5.4: Placement of Steel Reinforcement Small-Scale Specimen, Top View

\subsubsection{Casting}

All seven small-scale beams were cast on the same day, from the same batch of concrete. During the casting process 3 slump tests were conducted to verify the workability of the concrete mix. A total of thirty cylinders were cast to obtain the compressive strength of the samples at various time intervals after casting. The concrete was poured into the molds, a third of the height at a time, and vibrated for two minutes to ensure an even distribution of aggregate and paste throughout the reinforced concrete beams, and to avoid the occurrence of honeycombing during the casting process.

\subsubsection{Curing}

The samples were covered with damp burlap for the duration of the first seven days after casting, and sealed underneath a plastic tarp. This would ensure the proper curing of the samples in the critical first seven days. On the seventh day after the concrete reached approximately $80 \%$ of its strength, the beams were de-molded and tested. Figure 5.5 illustrates the seven small-scale beams on the day they were de-molded. 


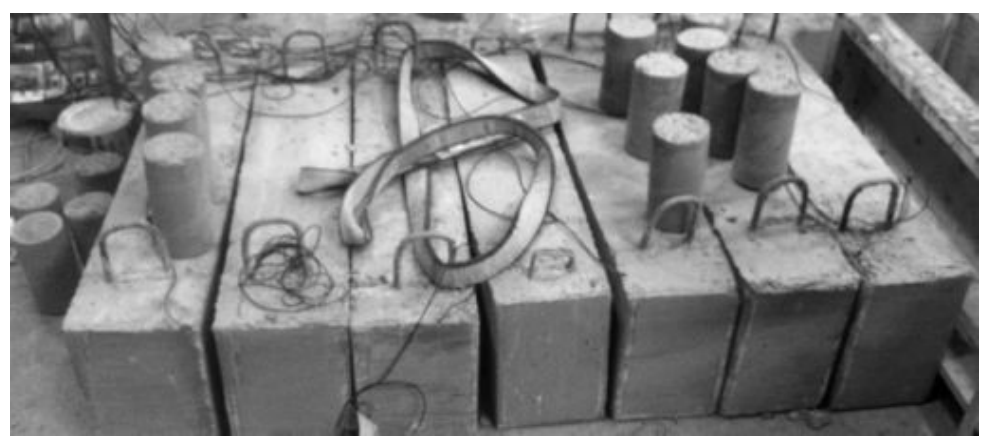

Figure 5.5: Small Scale Beams after De-Molding

\subsubsection{Loading Procedure}

The initiation and control of the flexural crack was achieved from the reinforced concrete design, as well as the loading procedure undertaken in the experiment. Each beam was loaded using the three-point load setup illustrated in Figure 5.6a. The concentrated load was applied directly at the center of the beam. A notch was created on the tension face of the beam directly underneath the location of the concentrated load to facilitate the initiation of the desired crack. The combination of a three-point load, and artificial fault, would guarantee the initiation of a crack directly at the beam's mid-span.
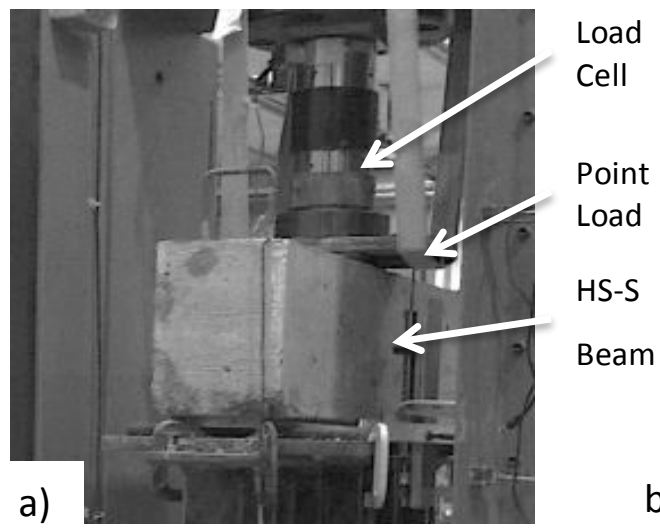

Figure 5.6: Loading Setup (a) Test Setup; (b) Three-Point Load Test Setup, Crack Gauge Location, and Notch Location

\subsubsection{Monotonic Beams (HS-S-M)}

The monotonic beams were loaded in $5 \mathrm{kN}$ load steps up to the failure load. The deflection of the beams, as well as the observed crack opening was recorded at every load step. 


\subsubsection{Cyclic Beams (HS-S-C)}

Each cyclic beam had its own loading procedure represented in Figure 5.7. Beam HS-S-C-1 was subjected to three load cycles (loading and unloading), one at $50 \mathrm{kN}, 100 \mathrm{kN}$, and 150 $\mathrm{kN}$; after which the member was loaded to its failure load. Beam HS-S-C-2 was exposed to a total of ten loading and unloading cycles. The beam was tested using two cycles of loading and unloading, at the following load steps: 50 kN, 70 kN, 100 kN, 150 kN, and $200 \mathrm{kN}$. The member was then loaded to its failure load. Beam HS-S-C-3 underwent a total of fifteen load cycles. The beam was exposed to three loading and unloading cycles at the, $50 \mathrm{kN}, 70$ kN, 100 kN, 150 kN, and 200 kN load steps, after which it was loaded to its failure load.

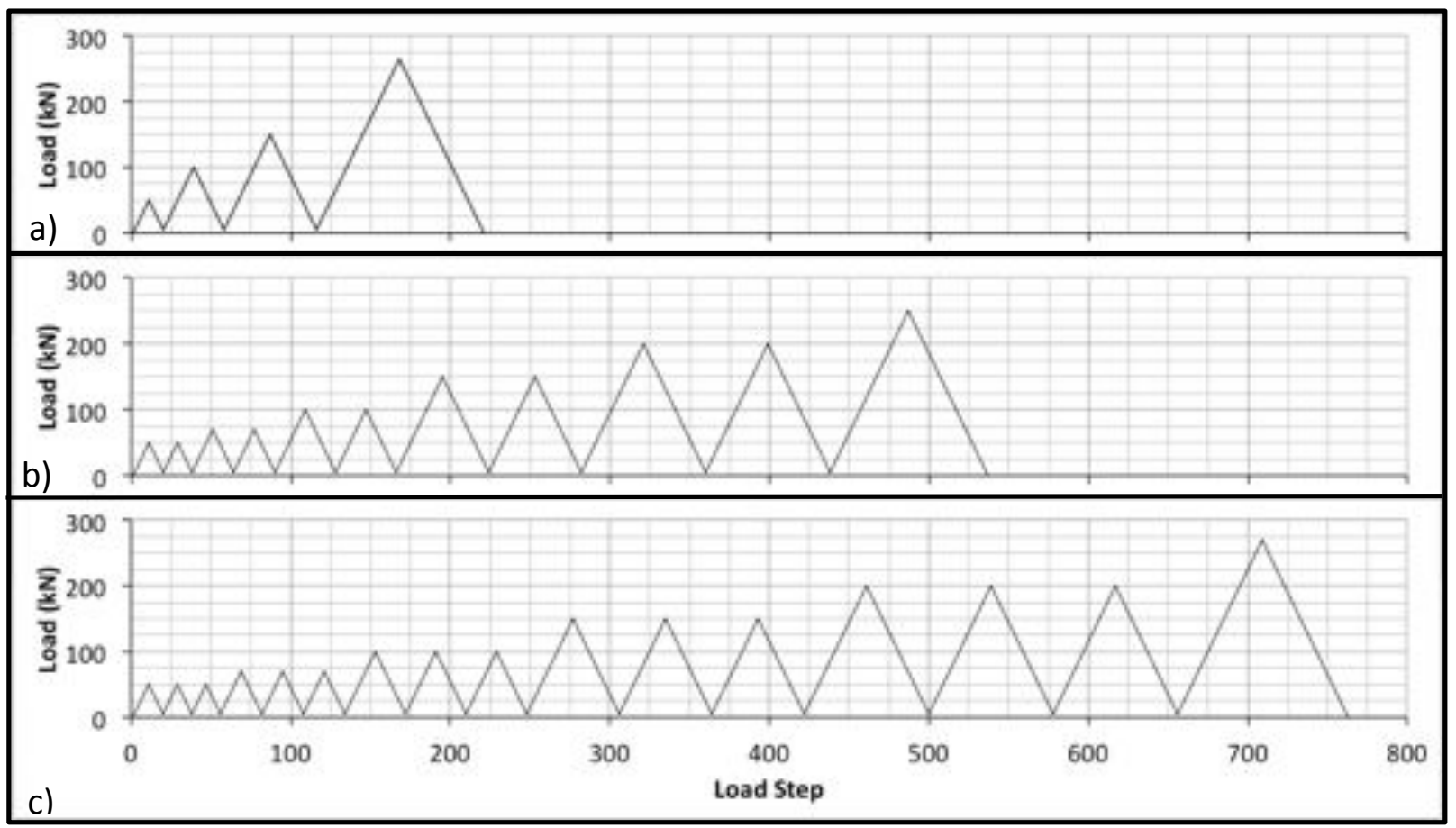

Figure 5.7: Loading Protocol Cyclic Beams (a) HS-S-C-1; (b) HS-S-C-2; (c) HS-S-C-3

\subsubsection{Measurement Devices}

\subsubsection{Crack Gauges}

Crack gauges were attached over the expected cracking region. These gauges were used to detect the initiation of cracking in the member and measure the crack width at each load 
step. One reading was recorded for every load step as to coincide with the data recorded by the piezo-sensors. These crack gauges were used to develop a relationship between, the strains and crack widths experienced by each member, and the signal output of the piezosensors. Two crack gauges were placed on either side of the beam $80 \mathrm{~mm}$ above its tension face as to measure the change in width of the crack at the same location that the piezosensors were installed to monitor the propagation of the crack. Figure $5.6 \mathrm{~b}$ demonstrates the location of the load application, as well as the location of the crack gauges. Both gauges were affixed to the face of the beam using anchor bolts, prior to the application of load. The crack gauges were used to monitor the strain experienced by the mid-section prior to the initiation of cracking, as well as the crack width after cracking.

\subsubsection{Piezo-Sensors}

Along with the two installed crack gauges, the cracking region was monitored using one pair of piezo-sensors. The sensors were placed $200 \mathrm{~mm}$ apart across the centerline of the beam as illustrated in Figure $5.8 \mathrm{~b}$.

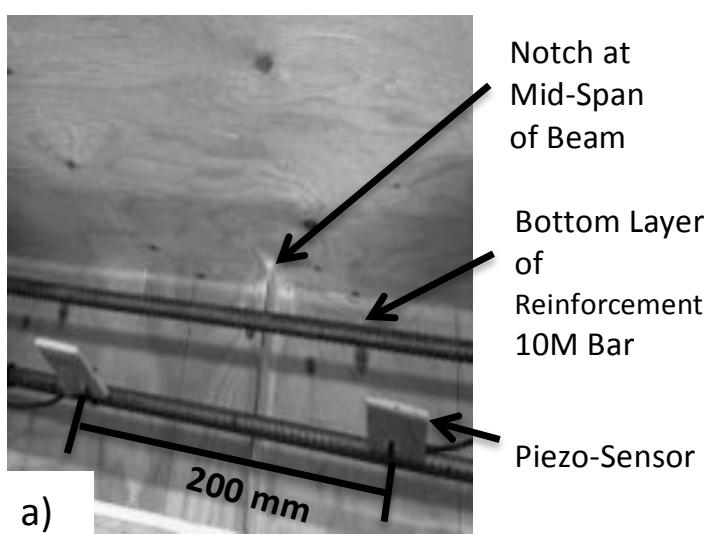

b)

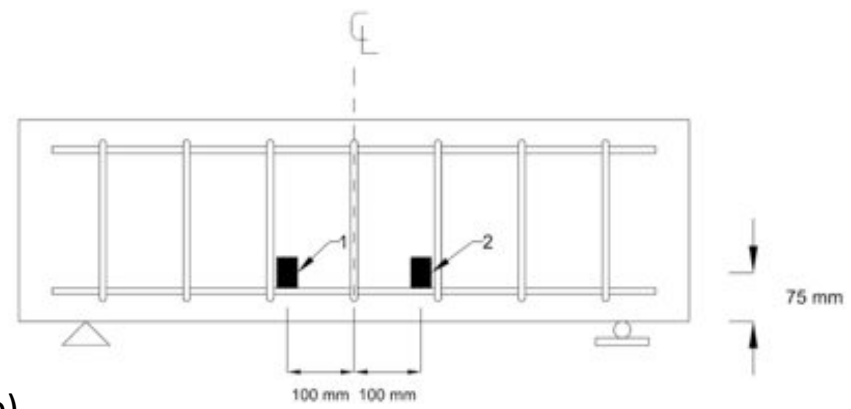

Figure 5.8: Placement of Piezo-Sensors in Small Scale-Beams; (a) Piezo Sensors in Small-Scale Beams; (b) Spatial Position of Piezo-Sensors in Small-Scale Beam

These sensors were attached directly to the tension reinforcement of each member, measuring the transmitted signal at $75 \mathrm{~mm}$ above the tension face of the beam. The region measured is precisely the same as that measured by the crack gauges, and would provide for a good comparison between the two results, facilitating the development of the 
relationship between the piezo-sensor signal and the concrete strain. Installing the piezosensors above the tension reinforcement would ensure the piezo-sensors measure only the concrete strain, and the beam's reinforcement does not interfere with the signal transmitted.

The placement of the piezo-sensors in the mold is presented in Figure 5.8a, along with the position of the induced saw cut. The piezo-sensors were attached using zip-ties to the tension reinforcement of each beam. The picture in Figure 5.8a demonstrates the region that was monitored by the piezo-sensors. This region is the area between the two piezosensors directly above the flexural reinforcement bar.

\subsection{Full Scale (HS-L-M)}

The HS-L-M series of beams were designed to determine the effectiveness of the embedded sensors as part of a complete health monitoring system. The piezo-sensors were arranged in each beam to monitor various cracking mechanisms (flexural and shear) that were expected during loading. By strategically placing the piezo-sensors in each beam, the location based monitoring characteristics of the piezo-system was studied, along with its application for predictive health monitoring. The results obtained were filtered, and processed into strains and crack widths using the developed model from the small-scale series of experiments. The validity of the developed model would be verified using embedded FBG fiber optic sensors at specific locations of the beam.

\subsubsection{Beam Geometry and Loading Procedure}

Both concrete beams had a total length of $4 \mathrm{~m}$ and cross-section of $350 \mathrm{~mm} \times 700 \mathrm{~mm}$. Although the two beams had the same geometry, the failure mode of each varied. Beam HS-L-M-1 was designed to fail in shear, whereas beam HS-L-M-2 was designed fail in flexure, and thus the location of the piezo-sensors differs between the two beams. Both beams were loaded using a hydraulic jack, in $25 \mathrm{kN}$ load steps under four point load setup up to their respective failure loads. 

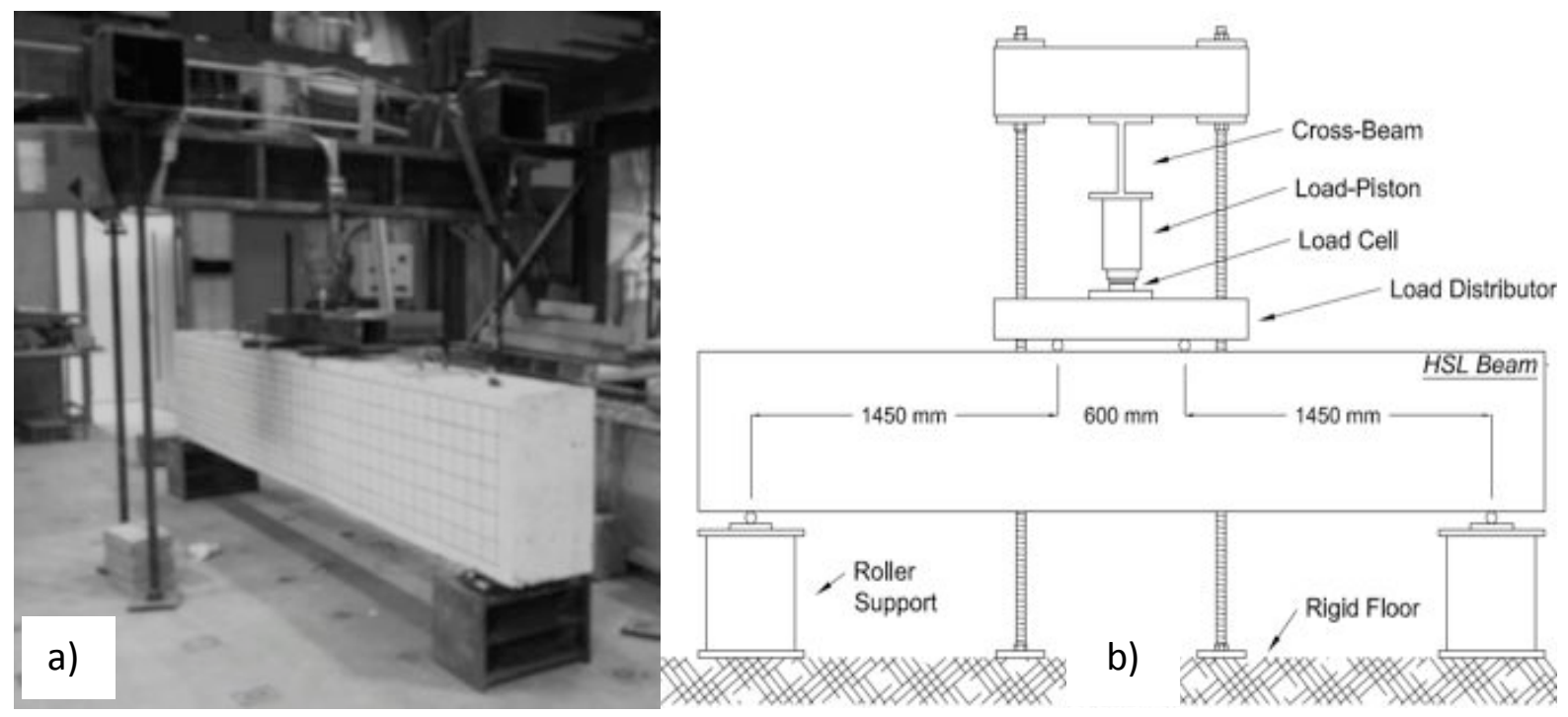

Figure 5.9: Test Setup HS-L-M Beams (a) Loading Setup; (b) Detail of Loading Setup

Figure 5.9 shows the experimental test setup used to test the full-scale beams. A load cell was placed directly underneath the loading piston to measure the applied load. This point load was transferred through a rigid load distributor into two-point loads $300 \mathrm{~mm}$ off center to either side of the beam. The point loads were $600 \mathrm{~mm}$ apart over the beam's center, creating a maximum moment arm of $1450 \mathrm{~mm}$. Three Linear Potentiometer Displacement Transducers (LPDT) were used to measure each beam's deflection. A LPDT was installed directly at the mid-span of the beam, and the other two were placed at each quarter-span. These were used to examine the beam's behavior during loading.

\subsubsection{Specimen HS-L-M-1}

\subsubsection{Test Specimen}

Beam HS-L-M-1 was designed to have the same amount of flexural reinforcement as HS-M$\mathrm{L}-2$, although the amount of shear reinforcement was reduced to an amount less than the minimum depicted by the Canadian code CSA-A23.3-04. The member's details are illustrated in Figure 5.10. The beams ultimate load capacity, limited in shear, was calculated to be $525 \mathrm{kN}$, or a maximum resistant moment of $380.63 \mathrm{kN} \cdot \mathrm{m}$. The cracking moment of the section was calculated to be $121.3 \mathrm{kN} \cdot \mathrm{m}$. 


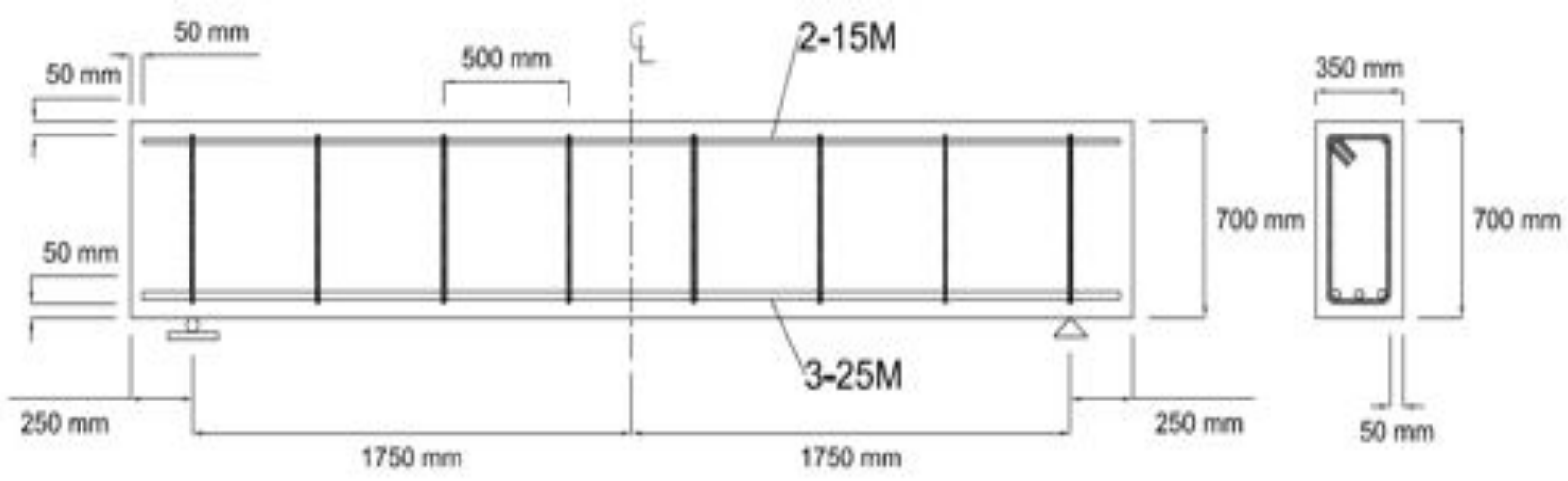

Figure 5.10: HS-L-M-1 Dimension and Reinforcement Detail

The large difference between the cracking and ultimate moments would ensure the aforementioned ductile behaviors. Beam HS-M-L-1 was expected to predominantly exhibit cracking at one third of the span. The cracking would initiate in the form of shear-flexural cracks at the early stages of loading. As the load continued to increase flexural cracks would appear at the mid-span. Beam HS-L-M-1 was equipped with ten piezo-sensors, as presented in Figure 5.11.

\subsubsection{Measurement Devices}

The ten piezo-sensors distributed along beam HS-L-M-1 were primarily oriented to monitor the initiation of shear cracking in the member. Figure 5.11a shows the position of the piezosensors among the steel reinforcement. Four sensors were placed in the compression zone of the beam, and six sensors were placed along the tension reinforcement. The top and bottom layers of piezo-sensors had a center-to-center separation of $433 \mathrm{~mm}$, and were distributed horizontally at a separation of $500 \mathrm{~mm}$, as shown in Figure 5.11a. Sensor pairs 45, 5-6, 6-7 check for the initiation of cracking development into the compression zone causing a member failure. The signal paths are illustrated in Figure 5.11b. Sensor pairs 1-2 and 9-10 were used to monitor only the strain at the support location, as cracking was not expected to happen for this region. These two sensor pairs act as a control for the experiment and ensure the sensor reading are accurate to the beam's behavior. No 
readings were taken between sensors 3 and 8 , as little significant cracking causing a member failure was expected over this region.

The primary focus of this investigation was sensor pairs 3-4 and 7-8. These sensor pairs were used to monitoring the initiation of shear cracks, and measure its width using the developed model from the small-scale series. In order to provide accurate results, sensors 3-4 and 7-8 had to be used in conjunction with the 2-3 and 8-9. As sensor pairs 2-3 and 8-9 monitor the critical shear section of the beam, it provides the early warnings of the initiation of a shear crack. The strain readings at the critical shear section, in conjunction with the strain and crack width readings obtained from sensor pairs 3-4 and 7-8 would provide the basis for a shear crack detection, and quantification system.

a)

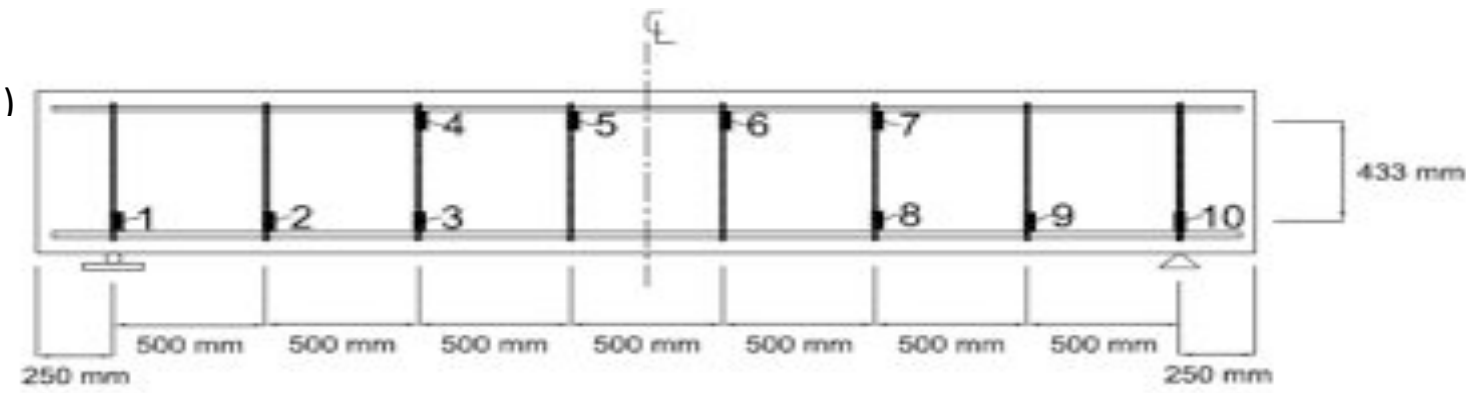

b)

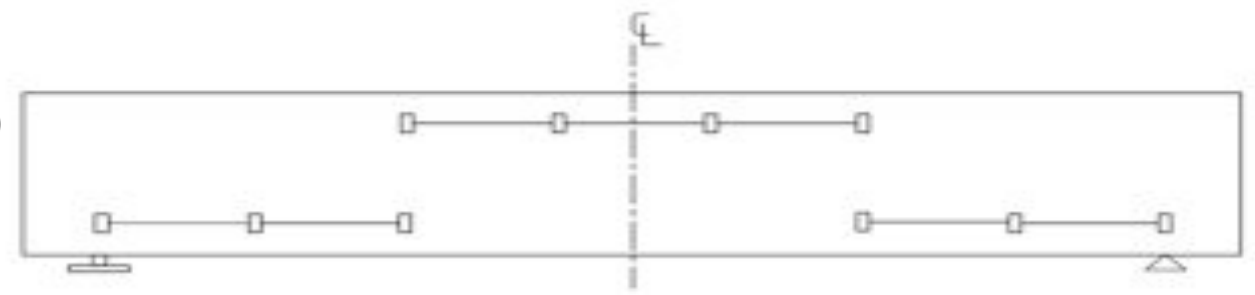

c)

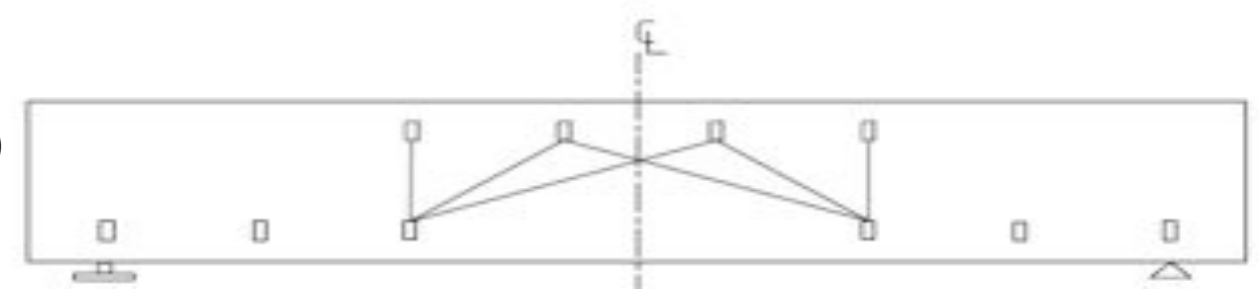

Figure 5.11: Piezo-Sensor Placement (a) Spatial Position of Piezo-Sensor in Beam HS-L-M-1; (b) Signal Paths for Flexural Control; (c) Signal Paths for Shear Control.

The midsection of the beam between sensors 3 and 8 was monitored only the crack height. Signals were transmitted between sensor pairs 3-5, 3-6, 8-5, and 8-6 as shown in Figure 5.11c. By monitoring the crack height, the behavior of the member could also be 
determined, as the member should not be exhibiting flexural activity. In this beam, lacking of the adequate shear reinforcement would cause little flexural cracking to appear in the midsection of the beam prior to the appearance of shear cracks. These sensors pairs were used to verify that the member is not exhibiting extensive flexural cracking, and confirm a shear failure of the member.

For this experimental program, the overall effectiveness of the system can be determined if it is able to: predict, detect and quantify the initiation of shear cracks, monitor the strain near the support location, and demonstrate a member failure by monitoring the initiation of cracking through the compression zone of the beam. The HS-L-M-1 beam was also equipped with FBG fiber optic strain gauges, and scanned using the MIRA 3D tomographer. The two techniques would help provide a comparison between the piezo-sensor system and to other better-established NDT methods.

\subsubsection{Specimen HS-L-M-2}

\subsubsection{Test specimen}

Beam HS-L-M-2 was designed to withstand a bending moment of $412.6 \mathrm{kN} \cdot \mathrm{m}$ (ultimate load of $569.1 \mathrm{kN}$ ), the beam was designed to withstand higher shear stresses, thus, guaranteeing flexural failure. Figure 5.12 shows the detailed drawing for beam HS-L-M-2.

A major consideration in the design of member HS-L-M-2 was the cracking moment. The effectiveness of the proposed sensors can only be judged by their ability to predict, detect, and quantify the initiation of cracking specific sections of the beam. To achieve the desired criteria, the beams cracking moment had to be much smaller than the ultimate moment of resistance to allow for the development of varying cracks along the beam's span. The large difference between the two moments would ensure the beam acts ductile and the piezosensors are exposed to a multitude of cracks that are evenly distributed along the beam's length. A cracking moment of $121.3 \mathrm{kN} \cdot \mathrm{m}$ was expected for the member in accordance to the CSA-A23.3-04. 


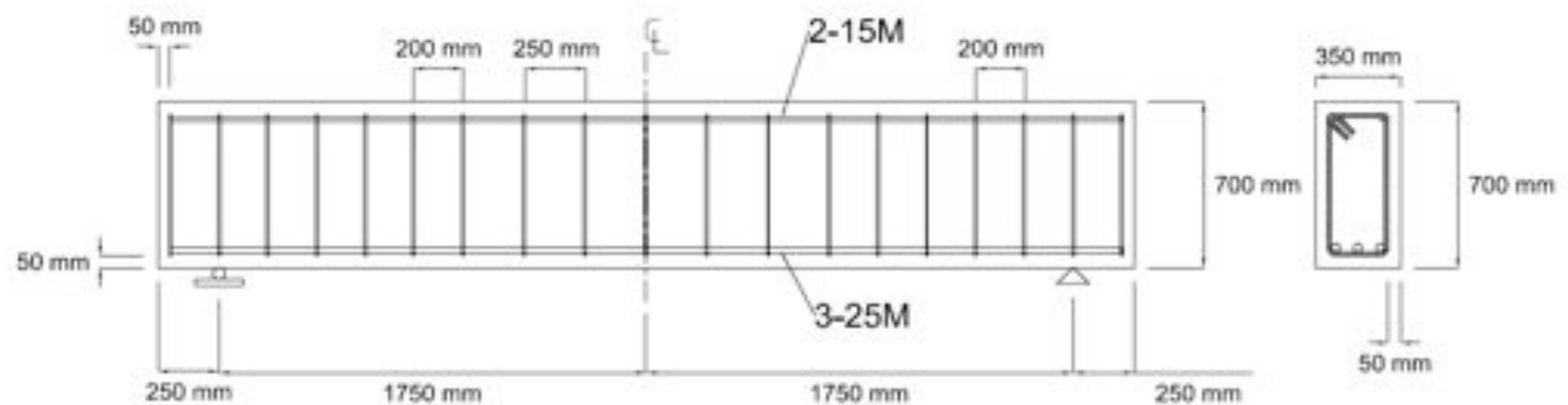

Figure 5.12: HS-L-M-2 Dimension and Reinforcement Detail

\subsubsection{Measurement Devices}

The distribution of piezo-sensors within a concrete member is very much governed by the idealized member behavior. A total of ten piezoceramic sensors were embedded into beam HS-L-M-2. The sensors were arranged in the concrete matrix in such a way that the initiation and distribution of flexural cracking within the beam would be primarily monitored, and to provide some information on the appearance of flexural-shear cracking, developed the later loading stages. Figure 5.13a shows the idealized model of the sensor placement among the steel reinforcement. As the piezo-sensors can both generate and receive stress wave, unidirectional signal paths were chosen for the investigation. Eight sensors were distributed along the tension side of the beam, as close to the outer most tension fiber of the concrete member, without compromising the sensors' signal transmissibility.

Signals transmitted through the combination of two piezo-sensors from sensors 1 through 8 were used in determining the initiation of flexural cracks within the member, and quantify the strains and crack widths experienced. The signal paths for the flexural control sensors are presented in Figure 5.13b. Sensors 9 and 10 in combination with sensors 1 through 8 were used for crack height measurements, and shear crack detection. The signal paths for these sensors are presented in Figure 5.13c.

Sensor pairs 9-3 and 10-6 were used to indirectly measure the development of the shear crack by adopting the model developed in the small-scale experimental program. As previously mentioned FBG fiber optic cables were also used to measure the strains at 
certain locations of this beam. The readings gathered by the FBG fiber optic sensors were used to confirm the effectiveness of the strain and crack width model developed by the small-scale experimental program. Sensor pairs 1-2 and 7-8 monitored the locations adjacent to the support. As not much cracking is expected in this region of the beam, these sensors act as a control for the experiment. The region monitored by the two pairs of sensors (1-2 \&7-8), is expected to undergo changes in strain with the application of load, although no cracking is expected to happen, and thus the sensors should reflect this.

a)

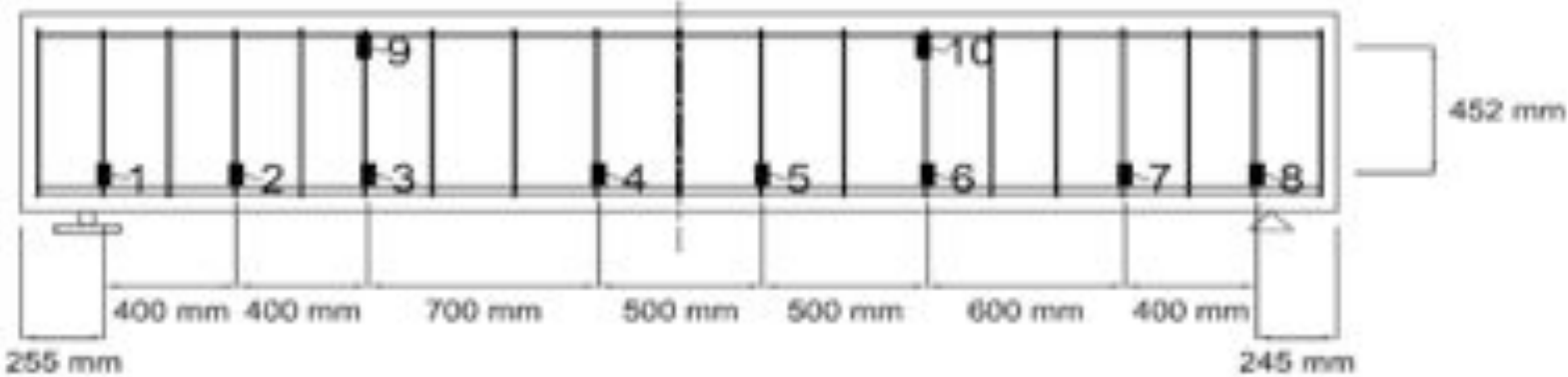

b)

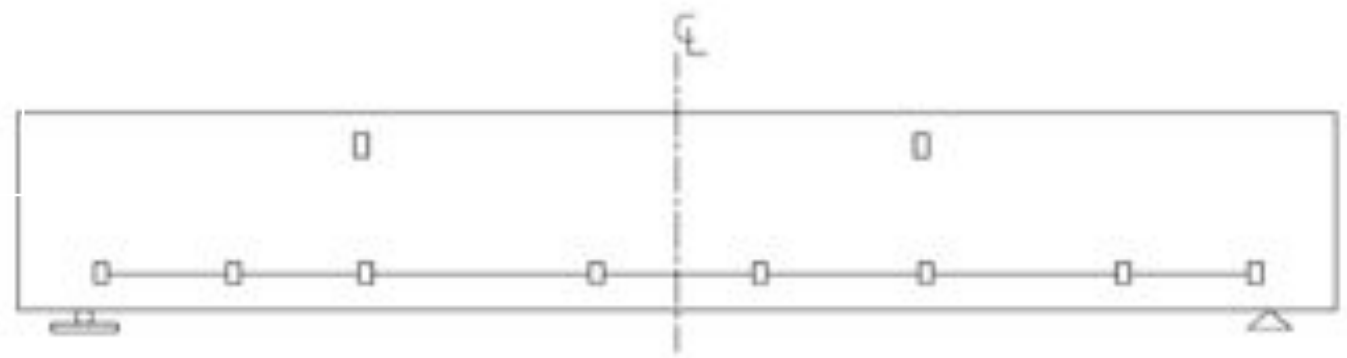

c)

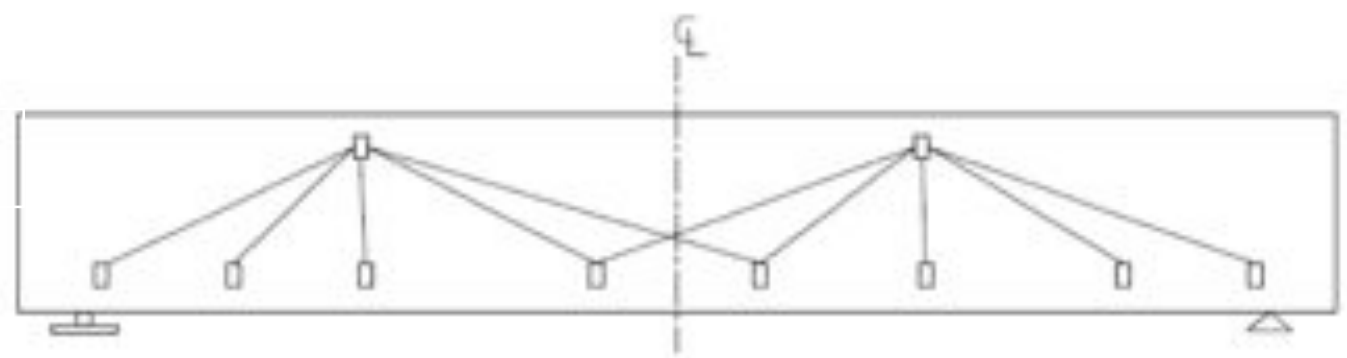

Figure 5.13: Piezo-Sensor Placement (a) Spatial Position of Piezo-Sensor in Beam HS-L-M-2; (b) Signal Paths for Flexural Control; (c) Signal Paths for Shear Control.

\subsubsection{Full-Scale Member Fabrication}

The following sections details the specimen fabrication process undertaken for the full-scale experimental program. The details for the construction of the formwork, placement of 
reinforcement, casting, and curing processes are presented herein. As previously stated the concrete used in the casting of these specimens was ordered, and not mixed on site having a minimum 28-day strength requirement of $50 \mathrm{MPa}$, and a slump exceeding 6 ".

\subsubsection{Formwork}

The formwork used in the casting of the full-scale beams was made out of wood, and is illustrated in Figure 5.14. The wooden molds consisted of five panels assembled together using nuts and bolts. The panels were made from 5-ply plywood of $3 / 8^{\prime \prime}$ thickness, strengthened with stiffeners made from timber with a cross-section of $2 "$ x 4 ". The vertical and lateral stiffeners would ensure that no distortion in the member's dimensions would occur during the casting process. The joints between each panel were water sealed using silicone, as to keep the moisture of the concrete confined in the mold.

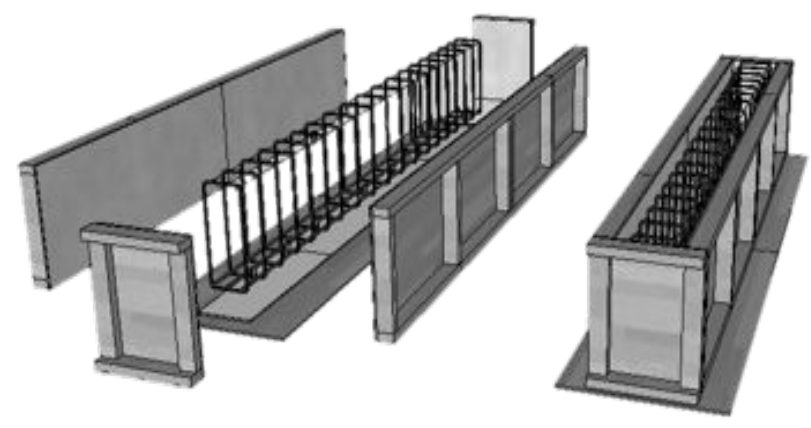

a)

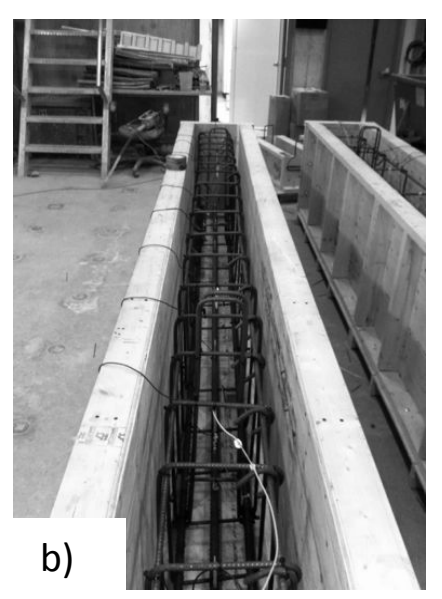

Figure 5.14: Formwork; (a) Assembly; (b) Pre-Casting

\subsubsection{Reinforcement}

Steel reinforcement bars of $450 \mathrm{MPa}$ yield strength were used. Prefabricated stirrups comprised the reinforcement cage of each member, and they were attached to the flexural reinforcement using zip-ties. Plastic seats, as to provide the $50 \mathrm{~mm}$ clearance with the bottom of the mold and create the clear concrete cover required, supported the three tensile flexural bars in the specimen. Two hangers were also fabricated and implemented 
as part of the reinforcement cage. The hangers were placed at the quarter-span of the beam, as to provide a means of transporting the member once it is cast and ready to test. The reinforcement cage for beam HS-L-M-2 is presented in Figure 5.15, as well as Figure 5.14b. The bars used as the tensile flexural reinforcement were $20 \mathrm{M}$, while the stirrups were made of $10 \mathrm{M}$ bar.
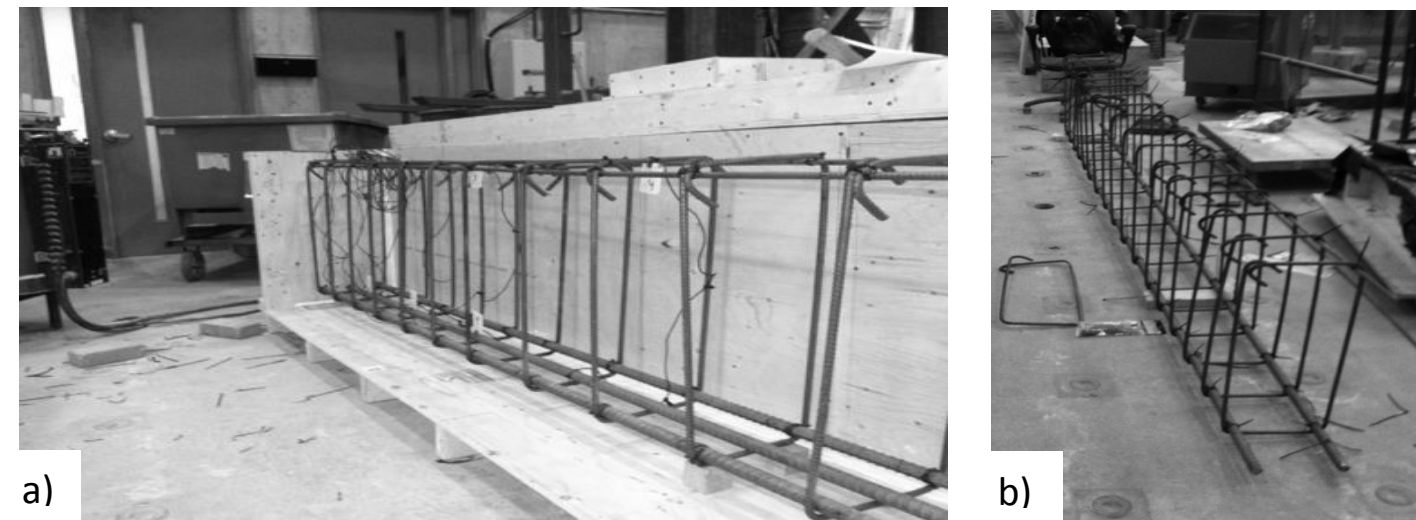

Figure 5.15: Steel Reinforcement Cage for Beam HS-L-M-2 Full-Scale Specimen; (a) Placed in Formwork; (b) Constructed

\subsubsection{Casting}

Prior to casting, the formwork was thoroughly cleaned and slightly oiled with form release. The two full-scale beams were cast on the same day, from the same concrete batch. During the casting process 3 slump tests were conducted to verify the workability of the mix. A total of twelve cylinders were cast to verify the compressive strength of the samples. The concrete was poured into the molds to a third of the height at a time, and vibrated for two minutes to ensure an even distribution of aggregate and paste through the confines of the formwork, as well as the steel reinforcing bars.

\subsubsection{Curing}

The samples were covered with damp burlap for the duration of the first seven days after casting, and sealed underneath a plastic tarp. This would ensure the proper curing of the samples in the critical first seven days. On the seventh day the beams were de-molded and tested. Figure 5.16 illustrates the full-scale member HS-L-M-2 the day it was demolded. 


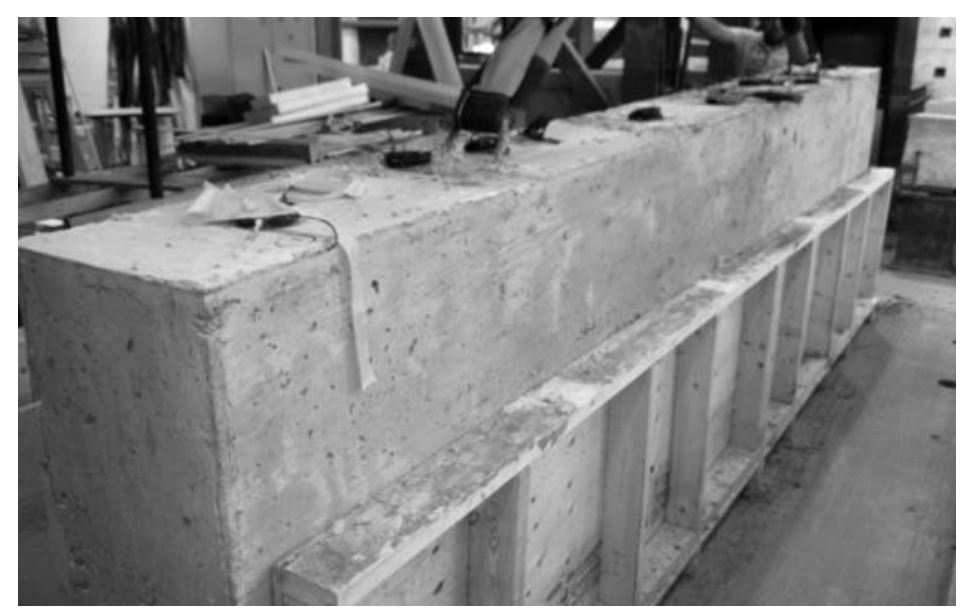

Figure 5.16: Full-Scale Member Demolding

\subsubsection{FBG Fiber Optic Sensors}

FBG fiber optic strain sensors are used in measuring concrete strains. The fiber optic sensors provide an accurate representation of the internal concrete strain, and from previous experimental work have proven to be very effective (Rizk et al. , 2012). These sensors were installed in both HS-L-M-1 and HS-L-M-2 beams. Each beam was equipped with one continuous cable containing five fiber optic sensors to monitor the concrete strain in the member. FBG sensor arrangement is presented in Figure 5.17.

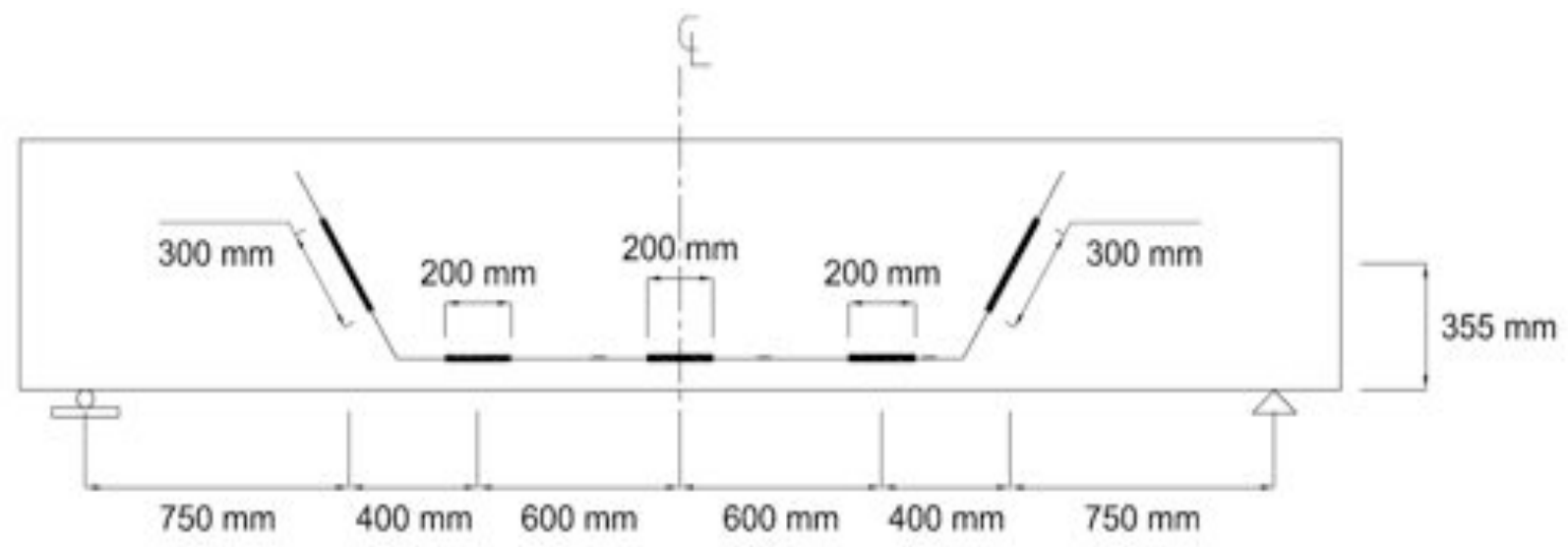

Figure 5.17: FBG Fiber Optic Sensors Spatial Position in the HSL Beam Series

Two $300 \mathrm{~mm}$ FBG sensors were arranged to monitor the strain, and capture the initiation of a shear crack in each member. The remaining three sensors had a gauge length of $200 \mathrm{~mm}$ to monitor the flexural cracking on the tension face of the beam. One sensor was installed 
to monitor the midsection of the beam detecting the initiation of flexural cracking for each member, while the remaining two sensors were placed $600 \mathrm{~mm}$ off center, near the quarter span of the beams to provide strain reading between specific piezo-sensor pairs. The five sensors were distributed symmetrically as to determine the consistency of results obtained form the both halves of the members, as shown in Figure 5.17. Three sensors were placed to monitor the flexural behavior and has a gauge length of $200 \mathrm{~mm}$ and two sensors were used to measure the strain across the shear cracks, having gauge lengths of $300 \mathrm{~mm}$.

\subsubsection{Arrangement of Fiber Optic Cable in Beam HS-L-M-1}

The arrangement of the fiber optic cable in beam HS-L-M-1 was installed to provide an accurate reading of the strains between five piezo-sensor pairs. Two shear sensors would monitor the initiation of the shear crack in the member, and the results of these would be compared to the estimated crack widths of piezo-pairs 4-3 and 7-8. The remaining fiber optic cables were used to monitor the section between sensors 3 and 8 . As no significant cracking was expected in this region, the sensors were primarily installed for confirmation of the beams behavior by validating the shear failure. The arrangement of the fiber optic cable among the piezo-sensors, in beam HS-L-M-1, is illustrated in Figure 5.18.

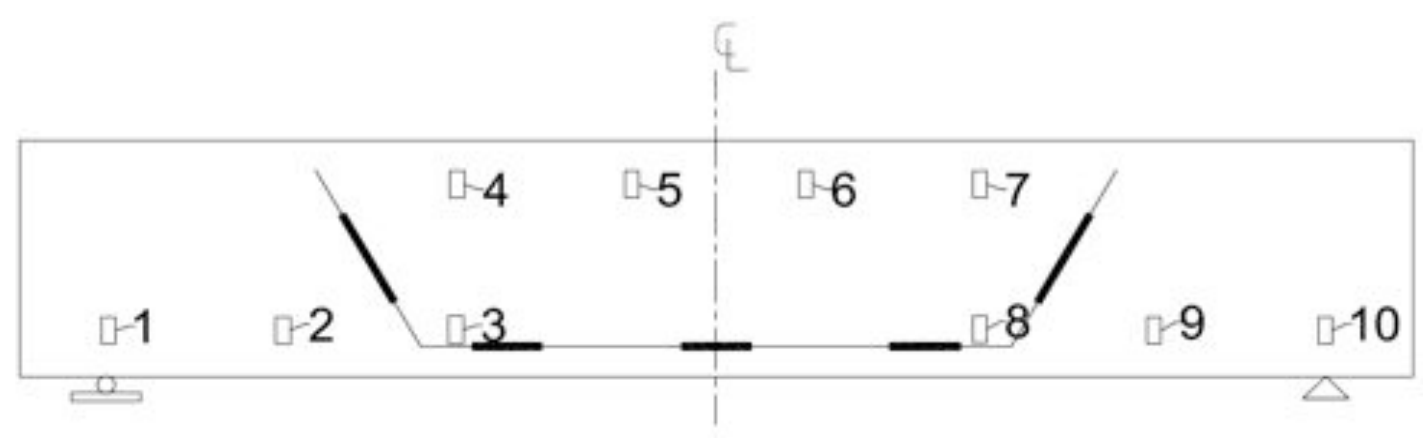

Figure 5.18: Arrangement of FBG Fiber Optic Sensors, and Piezo-Sensors in Beam HS-L-M-1

\subsubsection{Arrangement of Fiber Optic Cable in Beam HS-L-M-2}

The fiber optical cable in beam HS-L-M-2 was identical to that of beam HS-L-M-1. The comparison between the piezo-sensors, and the strain readings obtained from the fiber optic sensors would help to verify the validity of piezo-sensor technique. The arrangement 
of the FBG fiber optic cable among the piezo-sensors is presented in Figure 5.19. It can be noted that two sensors monitored the region between sensor pairs 9-3 and 10-6. These two sensors were used to detect the initiation of the shear crack and to measure its width. The remaining three sensors measured the strains in three locations along the tension side of the beam. These sensors measured the strains between piezo-sensor pairs, 3-4, 4-5, and 56. The three sensors monitoring the flexural strains of the beam were the only in-plane measurements taken between the piezo-sensors, thus these three sensors provide the basis for this study.

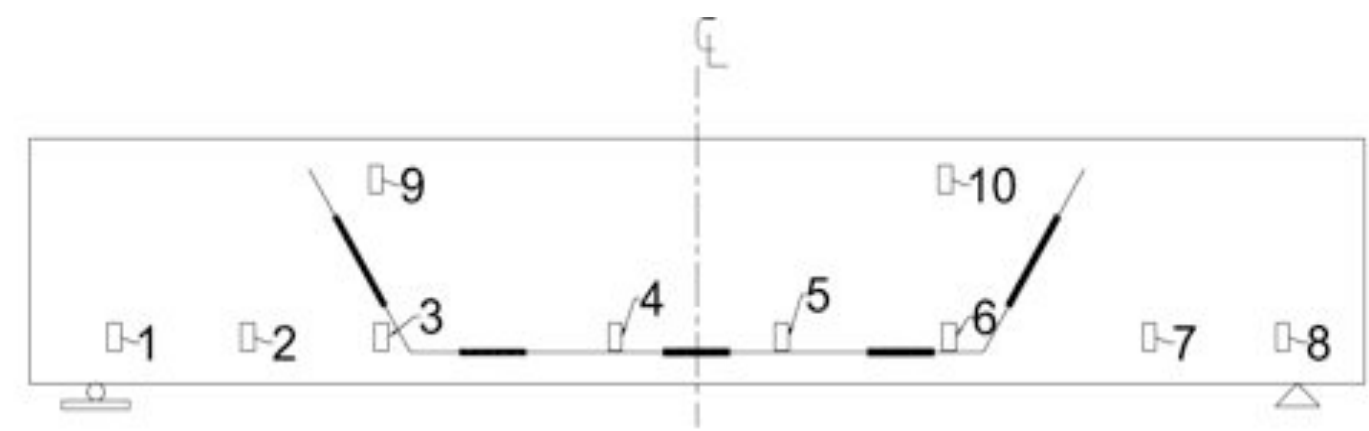

Figure 5.19: Arrangement of FBG Fiber Optic Sensors, and Piezo-Sensors in Beam HS-L-M-2

\subsubsection{MIRA 3D Tomographer}

The MIRA 3D tomographer was used to study the overall cracking behavior exhibited by both full-scale members. The tomographer is an external scanning device that provides a three-dimensional interpretation of the internal condition of concrete members. Using this device, both HS-L-M-1 and HS-M-L-2 were scanned at various load steps to help in mapping the propagation of cracking within each member. The readings were taken in steps of 100 $\mathrm{mm}$ along the span, and height of the beam. The symmetry of the beams was also used to limit the scanning surface to half of the beams length. As the tomographer will detect cracks that are perpendicular to the scan direction, and does not provide much information about cracks that are parallel, reading had to be taken in both the horizontal and vertical direction. Figure 5.20 demonstrates the scans taken along the length of the beam, and Figure 5.21 demonstrates the reading taken along the height of the beam. The MIRA 
tomographer has a scanning surface of $100 \mathrm{~mm}$ in the short direction and ranging from 400 $600 \mathrm{~mm}$ orthogonal to the scan direction depending on the settings used.

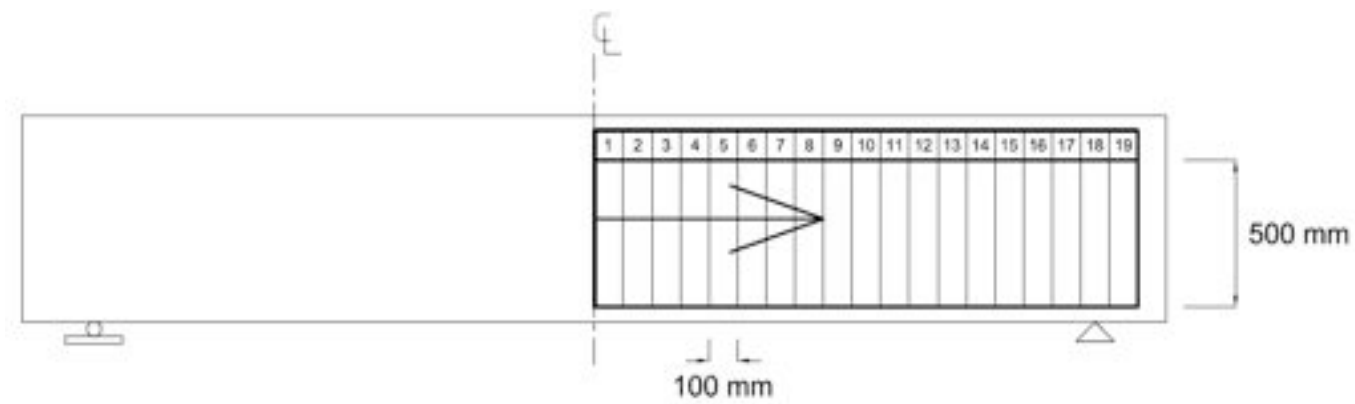

Figure 5.20: Vertical Scan Surface Using the MIRA 3D Tomographer

In the case of the vertical scanning 19 readings were taken along the length of the beam. The step width used for the readings was $100 \mathrm{~mm}$ leading to an overall scan surface having a length of $1900 \mathrm{~mm}$, and width of $500 \mathrm{~mm}$. The arrow in Figure 5.20 represents the direction of the scan.

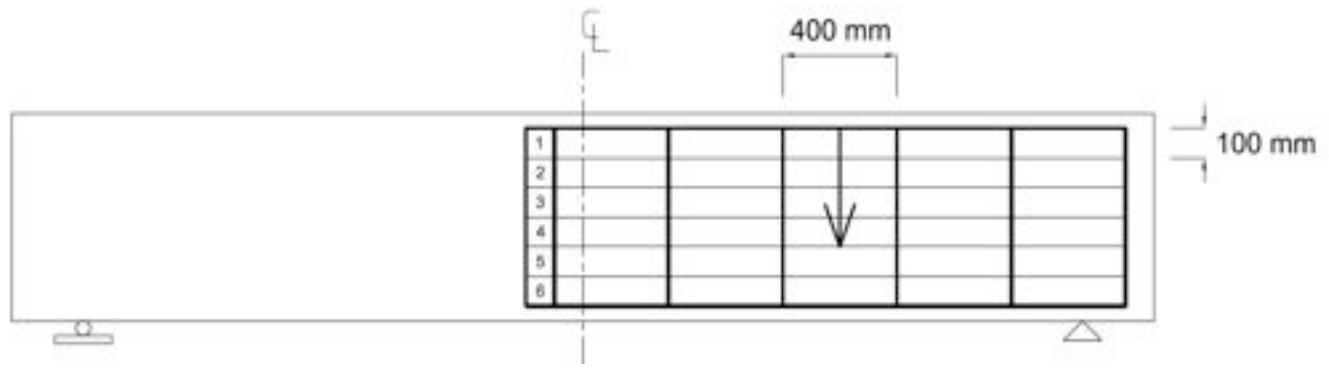

Figure 5.21: Horizontal Scan Surface Using the MIRA 3D Tomographer

In the case of horizontal scanning, the readings along the height of the beam were taken in five separate strips. The reading surface width of $400 \mathrm{~mm}$ was used and reading step of 100 $\mathrm{mm}$. The scanned surface is illustrated in Figure 5.21. Each reading strip was comprised of six individual readings, and the arrow in Figure 5.21 represents the direction of the scanning process. These sets of readings would capture the flexural cracking in the member, and provide an accurate map of their location. The information provided by the tomographer can be compared to the location-based readings provided by the proposed piezo-sensors. This comparison would provide a bridge between the more conventional external nondestructive ultrasonic testing methods, and the proposed internal piezoceramic system. 


\section{Chapter 6 |}

\section{Experimental Results and Analysis}

The experimental program consisted of both of the small-scale (HS-S), and full-scale (HS-L) test specimens, and the test results of these are presented herein. This section begins by presenting the results obtained by the small scale experimental tests, and applying these findings to the large scale test specimens to establish the capabilities of the piezo-sensors system as a comprehensive non-destructive solution. The piezo-sensors primary function will be the detection of cracking within a reinforced concrete member at critical sections. The test results obtained from the piezo-sensors are presented in terms of an HMI value, previously discussed, that will establish the basis of the system as an active non-destructive 
technique. A mean of interpreting the HMI values for predicting, detecting and quantifying the appearance of cracking in a reinforced concrete member is discussed in depth.

In this experimental program, nine specimens were tested, seven small-scale beams and two full-scale beams. The results presented for each member consist of: the member's behavior during loading, readings obtained from the piezo-sensors in terms of $\mathrm{HMI}$, followed by the results obtained from the strain measurement equipment. The results from both the strain measurement instruments, and the piezo-sensor system, are compared and analyzed to establish the abilities, and limitations of the proposed non-destructive system. The system is primarily analyzed for its ability to predict the occurrence of cracking within the concrete specimens, and establish a benchmark that can differentiate between a cracked and un-cracked section. This analysis is carried out on an individual member basis, and will be presented accordingly. The results obtained from the small-scale members are then analyzed as to provide a means for quantifying the induced damage as a measure of crack width.

The small-scale beams are used to determine two relationships between the piezo-sensors' $\mathrm{HMI}$ output readings, and the strain readings obtained from the crack gauges. The individual relationships between the HMI values and strain readings obtained from the installed crack gauges are presented for the monotonic and cyclic small-scale specimens individually. The two relationships are then converted in terms of HMI-Crack Width and compared with the direct crack width measurements obtained from the crack gauges in both experimental programs. The comparison will provide the most effective relation for predicting the effective crack width as suggested by each of the seven small-scale specimens, and to develop a universal HMI-Crack Width model that can be applied to the non-destructive evaluation of any structure. The proposed model is then verified using the results obtained from the large-scale specimens.

The proposed crack width model is used to determine the crack widths observed in the large-scale beams and is compared to the readings obtained from the strategically placed FBG fiber optic sensors. By adapting the proposed model to the large-scale beams, the 
piezo-sensor system can be considered as an effective health monitoring technique of reinforced concrete structures.

\subsection{Small-Scale Early Strength Prediction}

An investigation was carried out for quantifying the development of concrete's early strength using the piezo-sensors, on the small-scale experimental beams. The methodology of this analysis was presented in Section 4.5, and the analysis carried out is presented in this section. The section will illustrate the results obtained using the piezo-system, in comparison to the results obtained from multiple standard concrete cylinders tested during the first seven days after concrete casting (for results see Appendix A). The concrete ordered for the small-scale beams was specified to have a minimum 56-day compressive strength of $60 \mathrm{MPa}$. The results obtained from the standard concrete cylinders for the first 28-days are presented in Figure 6.1.

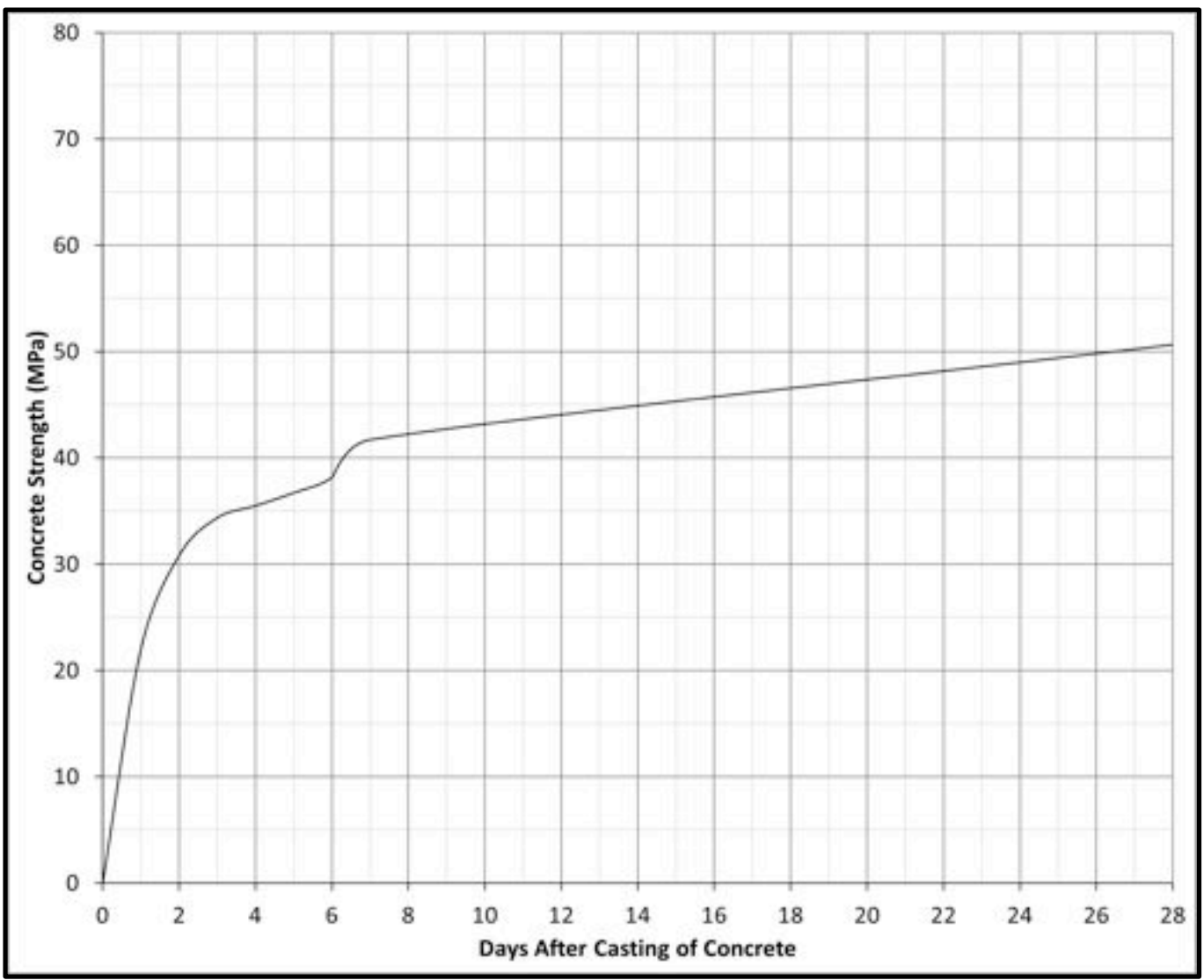

Figure 6.1: Compressive Strength Based on Standard Concrete Cylinders 
Table 2: Sample Spectral Shift Results from Beam HS-S-M-2 Based on Frequency Domain (kHz)

\begin{tabular}{c|c|c|cccccc}
\hline $\begin{array}{c}\text { Control } \\
\text { Points }\end{array}$ & Initial & Day & $\mathbf{2}$ & $\mathbf{3}$ & $\mathbf{4}$ & $\mathbf{5}$ & $\mathbf{6}$ & $\mathbf{7}$ \\
\cline { 2 - 8 } & $\mathbf{3 : 3 0}$ & Time & $2: 53$ & $2: 21$ & $2: 34$ & $2: 37$ & $2: 25$ & $2: 37$ \\
\hline 1 & $\mathbf{5 4 . 9 3}$ & & 59.40 & 59.51 & 61.00 & 61.20 & 61.50 & 62.50 \\
2 & $\mathbf{6 6 . 3 8}$ & & 72.30 & 73.24 & 74.00 & 75.00 & 75.15 & 75.51 \\
3 & $\mathbf{7 3 . 2 4}$ & & 78.12 & 79.80 & 80.10 & 80.30 & 80.40 & 80.60 \\
4 & $\mathbf{7 5 . 5 3}$ & & 82.30 & 82.40 & 83.20 & 83.20 & 83.80 & 84.40 \\
5 & $\mathbf{8 6 . 9 8}$ & & 93.84 & 95.00 & 96.00 & 96.50 & 96.80 & 97.00 \\
6 & $\mathbf{1 0 0 . 7 1}$ & & 108.00 & 109.50 & 110.10 & 109.50 & 111.00 & 111.85 \\
7 & $\mathbf{1 1 9 . 0 2}$ & & 128.17 & 130.00 & 131.00 & 132.50 & 132.80 & 135.00 \\
\hline
\end{tabular}

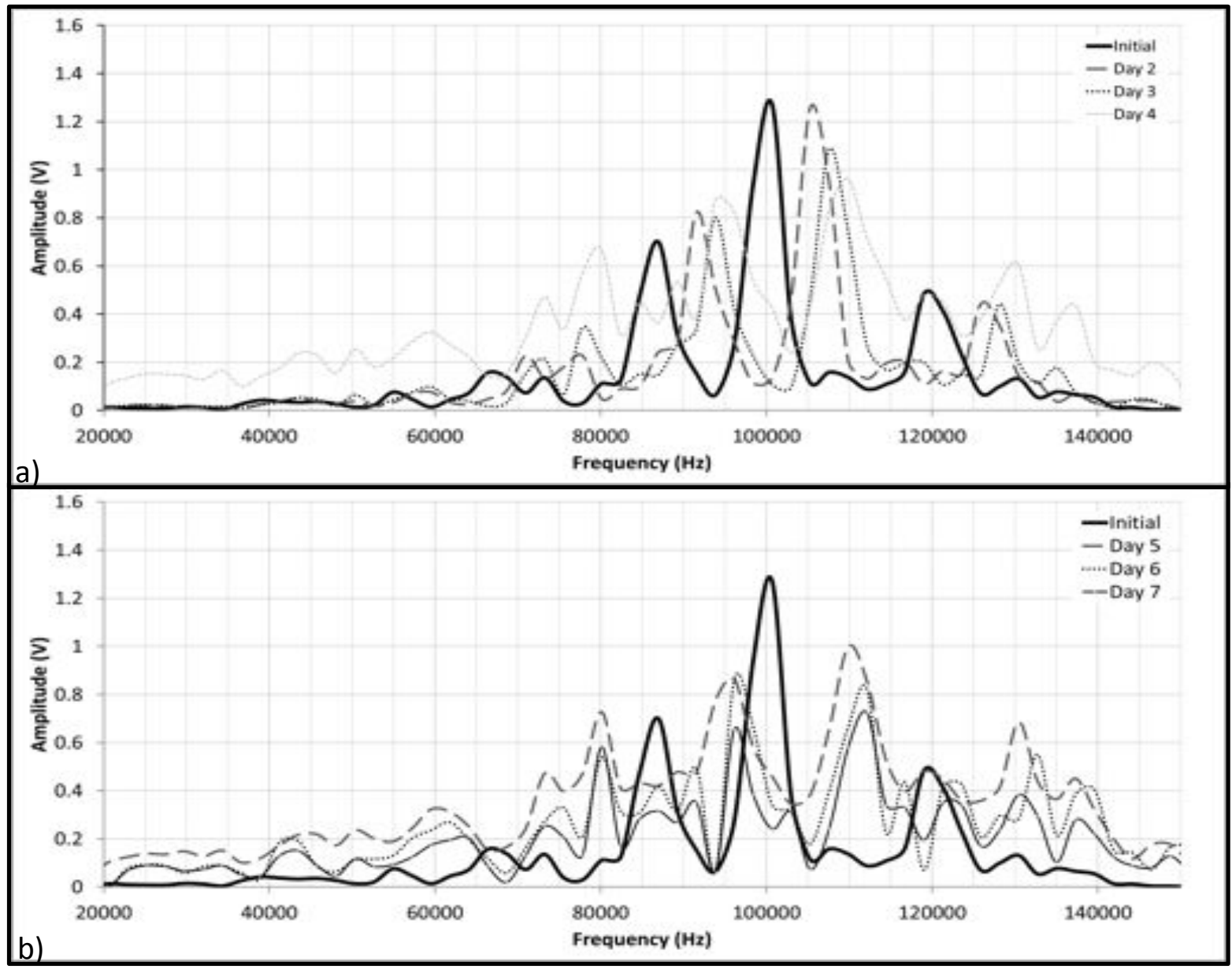

Figure 6.2: Sample Spectral Shift Beam from Frequency Domain of HS-S-M-2; (a) Day 1-4; (b) Day 5-7 
Three standard $100 \mathrm{~mm} \mathrm{X} 200 \mathrm{~mm}$ concrete cylinders were tested in 24-hour intervals, for the first seven days after casting the small-scale beam. The average compressive strength of the three cylinders was used to represent the compressive strength of the four monotonic small-scale beams investigated. This methodology of determining concrete's compressive strength coincides with the more conventional means of determining the properties of cast concrete on site, and provides a good means of comparison with the piezo-ceramic system.

The vibration characteristic of the four concrete beams was taken three times daily, for the first seven days after casting, to accurately measure the natural frequencies of the concrete matrix. A reading would be conducted prior to testing the standard cylinder samples, and two were taken after. The results of the three vibration characteristics obtained were compared for variations, and if a deviation was noticed between the vibration characteristic before and after the concrete cylinders were tested, the resulting natural frequencies were averaged between the three samples as to best represent the sample. Specific points, representing the highs and lows of the spectrum, were monitored through the development of the concrete's early strength. The shift in the spectrum was measured using these specific points, or natural frequencies, as to relate the results back to the compressive test results obtained from the concrete cylinders.

Figure 6.2 demonstrated the vibration characteristic obtained between the two piezosensors in beam HS-S-M-2. Figure 6.2a represents the readings obtained between days 2 and 4 , and Figure $6.2 \mathrm{~b}$ represents the reading obtained between days 5 and 7; where the initial reading represents the vibration characteristic of the mix 24 hours after casting. Beam HS-S-M-2 will be used as a sample to explain the steps undertaken in the analysis of all four beams.

The points monitored on the vibration-characteristic curve are presented in Table 2. A total of seven points were tracked through the seven days on the vibration characteristic curve, and the resulting shifts are illustrated in Figure 6.2, and summarized in Table 2. As the proposed method of determining the early strength propagation is relative to the compressive strength 24 hours after casting, the initial condition if presented separate, and the results for the following six days are compared to this initial condition. 
Table 3: Normalized Spectral Shift Beam HS-S-M-2

\begin{tabular}{c|c|cccccc}
\hline $\begin{array}{c}\text { Control } \\
\text { Points }\end{array}$ & Day & $\mathbf{2}$ & $\mathbf{3}$ & $\mathbf{4}$ & $\mathbf{5}$ & $\mathbf{6}$ & $\mathbf{7}$ \\
\cline { 2 - 7 } & Time & $2: 53$ & $2: 21$ & $2: 34$ & $2: 37$ & $2: 25$ & $2: 37$ \\
\hline 1 & & 1.08134 & 1.08333 & 1.11047 & 1.11411 & 1.11957 & 1.13778 \\
2 & & 1.08925 & 1.10345 & 1.11487 & 1.12993 & 1.13219 & 1.13762 \\
3 & & 1.06660 & 1.08954 & 1.09363 & 1.09636 & 1.09773 & 1.10046 \\
4 & & 1.08962 & 1.09094 & 1.10153 & 1.10153 & 1.10948 & 1.11742 \\
5 & & 1.07895 & 1.09227 & 1.10376 & 1.10951 & 1.11296 & 1.11526 \\
6 & & 1.07241 & 1.08730 & 1.09326 & 1.08730 & 1.10220 & 1.11064 \\
7 & & 1.07692 & 1.09226 & 1.10066 & 1.11327 & 1.11579 & 1.13427 \\
\hline Average & & 1.06119 & $\mathbf{1 . 0 8 0 9 2}$ & $\mathbf{1 . 0 9 2 0 8}$ & $\mathbf{1 . 1 0 3 0 4}$ & $\mathbf{1 . 1 0 7 5 3}$ & $\mathbf{1 . 1 1 3 6 0}$ \\
Shift & & & & & & & \\
\hline
\end{tabular}

Table 4: Summary of Average Spectral Shift of Each Beam

\begin{tabular}{c|c|cccccc}
\hline Beam & Day & $\mathbf{2}$ & $\mathbf{3}$ & $\mathbf{4}$ & $\mathbf{5}$ & $\mathbf{6}$ & $\mathbf{7}$ \\
\hline HS-S-M-1 & & 1.09120 & 1.10823 & 1.11178 & 1.11867 & 1.12693 & 1.12388 \\
HS-S-M-2 & & 1.08092 & 1.09208 & 1.10304 & 1.10753 & 1.11360 & 1.12487 \\
HS-S-M-3 & & 1.08582 & 1.10076 & 1.10597 & 1.11325 & 1.11936 & 1.12424 \\
HS-S-M-4 & 1.08887 & 1.09820 & 1.10626 & 1.11383 & 1.11742 & 1.11857 \\
\hline $\begin{array}{c}\text { Average } \\
\text { Shift }\end{array}$ & & $\mathbf{1 . 0 8 6 7 0}$ & $\mathbf{1 . 0 9 9 8 2}$ & $\mathbf{1 . 1 0 6 7 6}$ & $\mathbf{1 . 1 1 3 3 2}$ & $\mathbf{1 . 1 1 9 3 3}$ & $\mathbf{1 . 1 2 2 8 9}$ \\
\hline
\end{tabular}

Table 5: Average Spectral Shift vs. Concrete Compressive Strength $\left(f_{c}^{\prime}\right)$

\begin{tabular}{c|c|cccccc}
\hline & Day & $\mathbf{2}$ & $\mathbf{3}$ & $\mathbf{4}$ & $\mathbf{5}$ & $\mathbf{6}$ & $\mathbf{7}$ \\
\hline $\begin{array}{c}\text { Average Spectral Shift } \\
\text { All Beams }\end{array}$ & & 1.08670 & 1.09982 & 1.10676 & 1.11332 & 1.11933 & 1.12289 \\
\hline $\begin{array}{c}\text { Concrete Compressive } \\
\text { Strength }\left(\mathrm{f}^{\prime}\right)\end{array}$ & & 30.81 & 34.35 & 35.48 & 36.70 & 38.13 & 41.69 \\
\hline
\end{tabular}


For measuring the spectral shift of the vibration characteristic of the beam through the seven days, a relative measure was employed. The change in frequency on each respective day was divided by the initial frequency reading as to give a normalized incremental increase in the frequency response of each beam. The results of the normalization of bean HS-S-M-2 are presented in Table 3. Here the results presented in Table 2, for days 2 through 7, were divided by the initial frequency response of the initial vibration characteristic, presenting a relative increase in the natural frequency values of the spectrum. The results for the seven control points are averaged, and provide an average spectral change in the vibration characteristic properties of beam HS-S-M-2, as yielded by the piezo-ceramic system.

The above analysis was carried out for each of the four concrete beams, and the average spectral change for each beam, from day 2 to day 7, is presented in Table 4. The spectral shifts of the four beams are then averaged between them as to provide one value of spectral shift for each for the six days that the beams were tested, and provide a direct correlation to the results obtained from the cylinder compressive strength values. From here, a universal relationship can be developed to predict the compressive strength of a concrete section based on the results obtained from the frequency response graphs provided by the piezo-system. Table 5 represents the average spectral shift of the four beams for any given day versus the corresponsive compressive strength obtained from the concrete cylinders.

Equation 6.1 represents the relationship obtained from the results presented in Table 5 . Here the compressive strength (MPa) of a concrete section on any given day $\left(\mathrm{f}^{\prime}{ }_{\mathrm{cd}}\right)$ is defined by the spectral shift on that specific day, and the compressive strength of the concrete 24 hours after casting. $F_{i}$ represents the natural frequency $(\mathrm{kHz})$ of the sample 24 hours after casting; $F_{d}$ represents the natural frequency $(\mathrm{kHz})$ on any given day; and $\mathrm{f}_{\mathrm{c} 1}^{\prime}$ represents the result of the compressive strength ( $\mathrm{MPa}) 24$ hours after the concrete is cast.

$$
{f^{\prime}}_{c d}=163.65\left(\frac{F_{d}}{F_{i}}\right)-\left(121.47+f_{c 1}^{\prime}\right)
$$


Table 6: Results for Compressive Strength Obtained from Piezo-Sensors Using Equation 5.1

\begin{tabular}{c|c|ccccccc}
\hline Beam & Day & $\mathbf{1}$ & $\mathbf{2}$ & $\mathbf{3}$ & $\mathbf{4}$ & $\mathbf{5}$ & $\mathbf{6}$ & $\mathbf{7}$ \\
\hline HS-S-M-1 & & & 30.57 & 35.11 & 37.90 & 38.48 & 39.61 & 40.96 \\
HS-S-M-2 & & 21.99 & 30.20 & 33.43 & 35.26 & 37.05 & 37.79 & 38.78 \\
HS-S-M-3 & & & 30.12 & 34.24 & 36.68 & 37.53 & 38.72 & 39.72 \\
HS-S-M-4 & & & 29.85 & 34.73 & 36.26 & 37.58 & 38.82 & 39.41 \\
\hline $\begin{array}{c}\text { Cylinder } \\
\text { Compressive } \\
\text { strength }\end{array}$ & & 21.99 & 30.81 & 34.35 & 35.48 & 36.70 & 38.13 & 41.69 \\
\hline
\end{tabular}

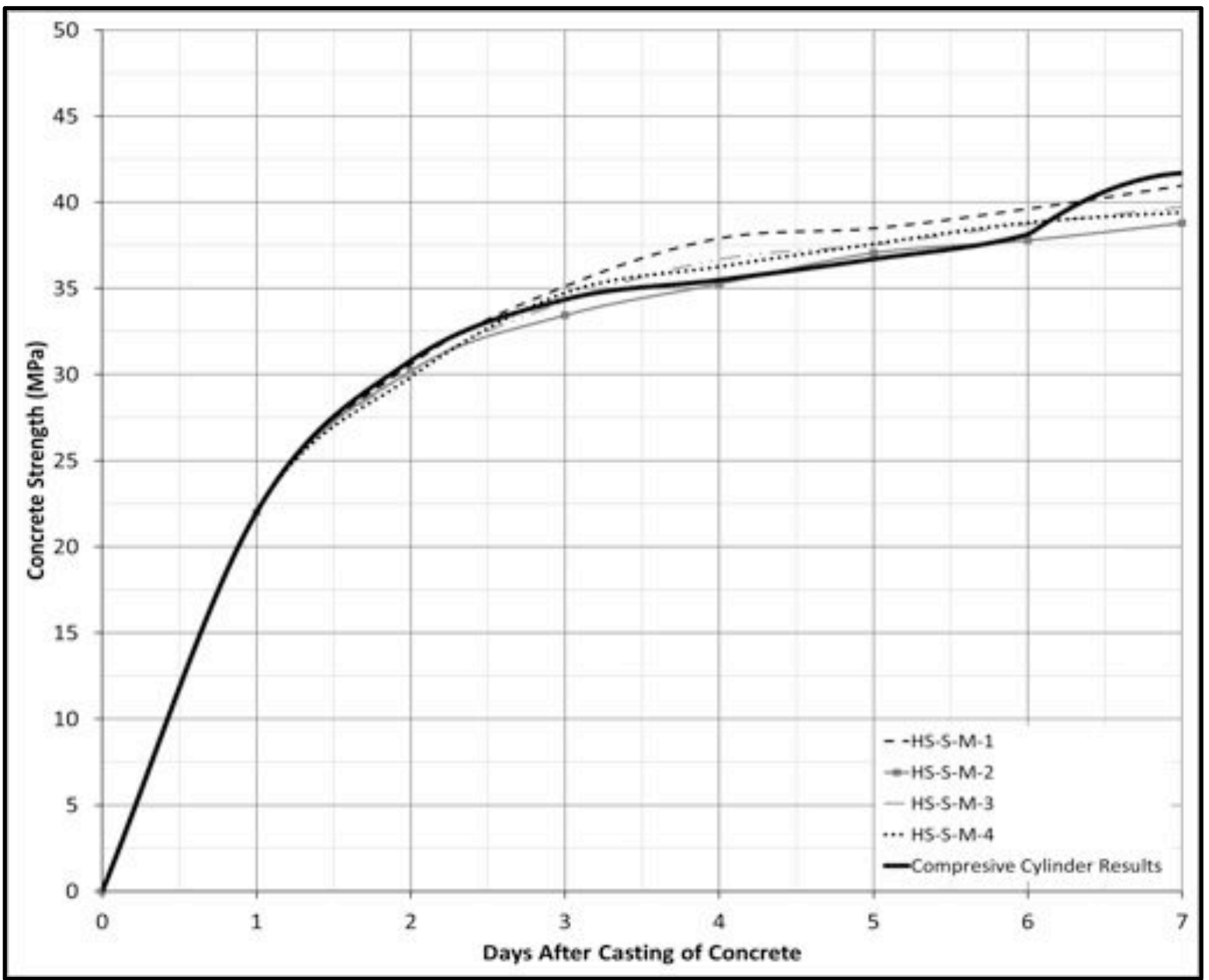

Figure 6.3:Early Compressive Strength Predictions Using Piezoceramic Sensors 
To verify Equation 6.1, the results of the spectral shifts obtained in Table 4 are processed using the equation into the respective compressive strengths as to compare to the results obtained from the standard cylinder tests, to those of the piezo-system. The results for the compressive strengths for each of the four beams, within the first seven days after casting, are presented in Table 6, and illustrated in Figure 6.3, along with the respective results obtained from the standard cylinder tests on the respective day. The results suggest Equation 6.1 is in very good agreement with the current standard for determining a concrete batches' compressive strength.

The results obtained from the analysis suggest that the piezo-system is a very useful tool for determining the internal compressive strength development of reinforced concrete members' within a building system. The piezo-system can provide reading of a member's compressive strength on a location basis, delivering a broader understanding of the buildings strength development, and minimizing the requirement of vast numbers of concrete cylinder tests.

\subsection{Small Scale Beams under Monotonic Loading (HS-S-M)}

The HS-S-M experimental programs consisted of four small-scale beams tested under monotonic load, and carry the designation "HS-S-M-\#". These members were each equipped with a pair of piezo-sensors, and a crack gauge on either face of the beam. The members were loaded up to their respective failure loads in load steps of $5 \mathrm{kN}$, and the results obtained from the measurement equipment is presented, along with the relationship between the crack gauge strain reading and $\mathrm{HMI}$ outputs that is later used to develop the desired HMI-Crack Width model. The visually observed cracking behavior of each member is also discussed for comparison.

\subsubsection{Member Behavior}

The four beams tested in this experimental program were designed to be identical, in geometry, reinforcement detailing, material properties, and loading procedure. The Load- 
Deflection graphs for the four members are presented in Figure 6.4, and exhibited similar behaviors when exposed to the described loading procedure. Table 7 presents the failure loads and the corresponding mid-span deflections, along with the cracking loads of each beam. Beam HS-S-M-1 had a failure load of $235 \mathrm{kN}$ at a deflection of $4.97 \mathrm{~mm}$; beam HS-SM-2 had a failure load of $250 \mathrm{kN}$ at a deflection of $4.70 \mathrm{~mm}$; beam HS-S-M-3 had a failure load of $250 \mathrm{kN}$ at a deflection of $5.25 \mathrm{~mm}$; and member HS-S-M-4, had a failure load of 260 $\mathrm{kN}$ at a deflection of $4.43 \mathrm{~mm}$. Overall the members proved to have comparable ductility that will be reflected in the results obtained from the crack gauge and piezo-sensors' readings. Beams HS-S-M-1 and HS-S-M-3 had very similar behaviors, where beam HS-S-M-3 is seen to be slightly stiffer than HS-S-M-1. Beam HS-S-M-4 demonstrated the stiffest behavior of the four beams by exhibiting the highest failure load with the lowest deflection.

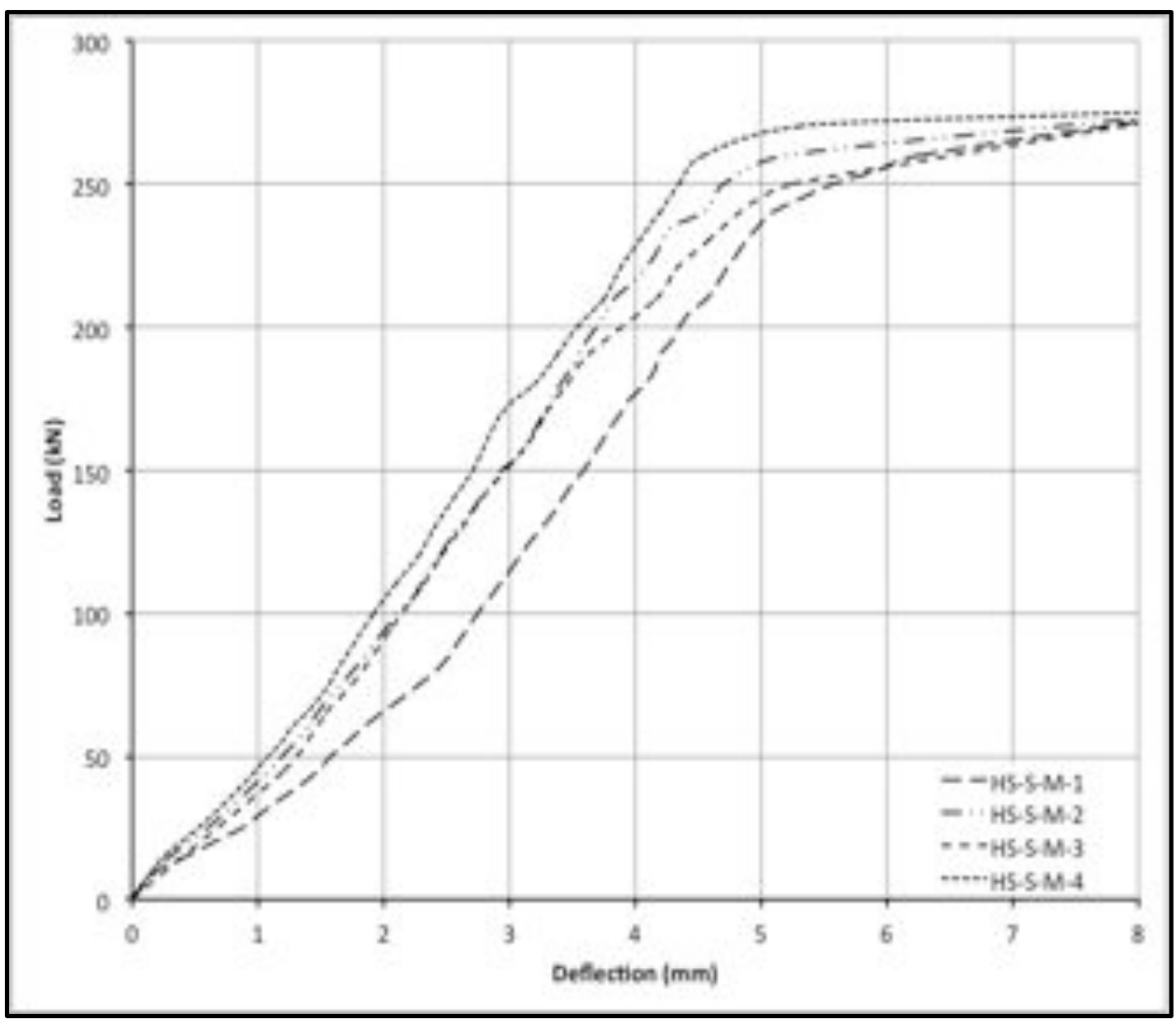

Figure 6.4: Load-Deflection Graphs, Small Scale Monotonically Loaded Members 


\subsubsection{Crack Gauge Readings}

The small-scale experimental beams were equipped with crack gauges that would measure the strain at the mid-span of the member, directly underneath the location of the applied load. The gauges report the measured strain between the two anchor positions, which can be converted to the effective crack width. Each member was equipped with a crack gauge on either face, and the results of these were averaged as to give a clear representation of the crack width at the middle of the beam where the piezo-sensors were monitoring. Although, some of the data obtained is limited, the agreement between the results of the 4 test specimens provides a clear representation of the strain experienced.
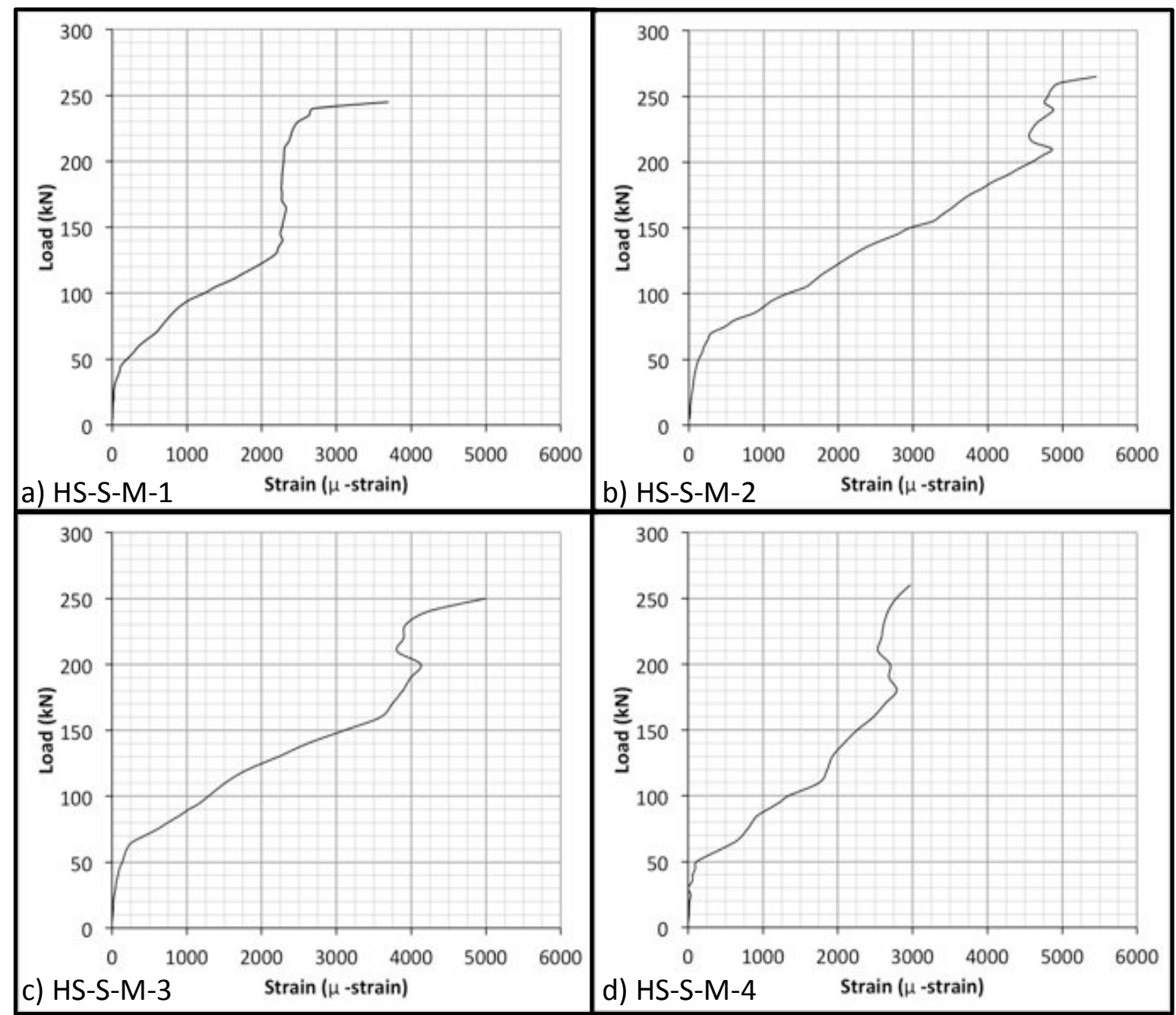

Figure 6.5: Load-Strain Reading from Crack Gauge Equipment Small Scale Monotonically Loaded Members; (a)HS-S-M-1; (b) HS-S-M-2; (c) HS-S-M-3; (d) HS-S-M-4 
The average Load-Strain graphs, obtained from the installed crack gauges, for each individual member are presented in Figure 6.5. The four members showed similar trends, and provide a clear representation of the members' cracking behavior. The cracking load of each member is determined using the readings obtained from the crack gauges as the member shows an abrupt change in the load-strain relationship as presented in Figure 6.5. An average value of the cracking strain of 110 micro strains was observed for the four tested beams as presented in Table 7.

The Load-Strain graph obtained from member HS-S-M-1 is presented in Figure 6.5a, where it can be seen the crack gauge was effective up to a strain of $2200 \mu$-strain. After the 2200 $\mu$-strain limit, a crack intercepted the location of one of the anchor bolts and the readings are not representative of the effective strain. The cracking load for member HS-S-M-1 is found from Figure $6.5 \mathrm{a}$ to be $45 \mathrm{kN} ; 30 \mathrm{kN}$ prior to it becoming visible to the naked eye. The Load-Strain results for beam HS-S-M-2 are presented in Figure 6.5b, providing clear measurements up to $4800 \mu$-strain. The cracking load of this member is found from the graph to be $50 \mathrm{kN}$. Beam HS-S-M-3 was found to have a cracking load of $50 \mathrm{kN}$. The LoadStrain graph is presented in Figure 6.5c, where the results of the crack gauge are presented to be accurate to $3750 \mu$-strain. Beam HS-S-M-4 proved to be the least ductile of the four beams tested, and is reflected by the readings obtained from the crack gauge. The cracking load of this member was found to be $55 \mathrm{kN}$ although the slope of the Load-Strain graph is seen to be much greater than that of the other members. The crack gauge of this member was effective up to $2790 \mu$-strain, from where the anchor bolt lost its bond to the concrete specimen. The cracking loads obtained from the crack gauges are very important in establishing the relationship between the $\mathrm{HMI}$ and strain, and will be further discussed in the analysis of the members.

The test results obtained from the crack gauges are used in developing the HMI-Strain for the monotonically loaded members. These strain readings, along with the Load-Deflection curves suggest a very good consistency between the behaviors of the four tested specimens, which must be reflected in the HMI readings. The strain readings provide a means of establishing a benchmark to indicate the appearance of the first crack within a 
member based on the $\mathrm{HMI}$ readings, as well as establish a means of predicting the occurrence of cracking in the reinforced concrete member.

Table 7: Summary of Results for Monotonic Small-Scale Members

\begin{tabular}{c|cccccc}
\hline $\begin{array}{c}\text { Member } \\
\text { Notation }\end{array}$ & $\begin{array}{c}\text { Failure } \\
\text { Load }\end{array}$ & $\begin{array}{c}\text { Failure } \\
\text { Deflection }\end{array}$ & $\begin{array}{c}\text { Observed } \\
\text { Cracking } \\
\text { Load }\end{array}$ & $\begin{array}{c}\text { HMI at } \\
\text { Observed } \\
\text { Crack Load }\end{array}$ & $\begin{array}{c}\text { Actual } \\
\text { Cracking } \\
\text { Load }\end{array}$ & $\begin{array}{c}\text { HMI at } \\
\text { Actual } \\
\text { Cracking } \\
\text { Load }\end{array}$ \\
\hline HS-S-M-1 & $235 \mathrm{kN}$ & $4.97 \mathrm{~mm}$ & $75 \mathrm{kN}$ & 0.61 & $45 \mathrm{kN}$ & 0.40 \\
HS-S-M-2 & $250 \mathrm{kN}$ & $4.70 \mathrm{~mm}$ & $65 \mathrm{kN}$ & 0.37 & $50 \mathrm{kN}$ & 0.26 \\
HS-S-M-3 & $250 \mathrm{kN}$ & $5.25 \mathrm{~mm}$ & $70 \mathrm{kN}$ & 0.48 & $50 \mathrm{kN}$ & 0.28 \\
HS-S-M-4 & $260 \mathrm{kN}$ & $4.43 \mathrm{~mm}$ & $60 \mathrm{kN}$ & 0.56 & $55 \mathrm{kN}$ & 0.29 \\
\hline
\end{tabular}

\subsubsection{Piezo-Sensors Results}

The embedded piezoceramic patches, for the small-scale experimental program, were installed to monitor the development of the crack across the mid-section of the member. The sensors provided a direct comparison between the strain readings obtained by the crack gauges, and the signals output from the piezo-sensors. The readings obtained from the crack gauges were used to determine the load at which each monotonically loaded member reached its cracking load. Knowing the cracking load of each member, a limit for the $\mathrm{HMI}$ can be established as to capture the initiation of cracking in the reinforced concrete member. The Load-HMI graphs for each of the four members are presented in

Figure 6.6 The four graphs triggered a similar trend. The results show a natural progression from a $\mathrm{HMI}$ of 0 to approximately 0.6 , from where, consistently for the four members, a sharp change in slope is present. This sudden change in slope shows that the relationship between the load and HMI is not linear but rather exponential, as it will be discussed later. The signal output is compared to the visually observed cracking loads, as to establish the system ability to determine the occurrence of the first crack within a member, well before it becomes visible on the beam's exterior. 
The response of the piezo-sensors in beam HS-S-M-1 is presented in Figure 6.6, and summarized in Table 7. The graph demonstrates an approximate linear response between the increase in $\mathrm{HMI}$ and load, up to a value of 0.6 , at which a sudden change in slope is noticed. Member HS-S-M-1 cracked at a load of $45 \mathrm{kN}$ from the crack gauge results, and visually observed at a load of $75 \mathrm{kN}$. The respective $\mathrm{HMI}$ readings at these specific loads are: 0.40 at $45 \mathrm{kN}$, and 0.61 at $75 \mathrm{kN}$. The results obtained from HS-S-M-1 show that the response of the piezo-sensors is very sensitive and linear in the un-cracked stages of the beam's loading program. This sensitivity makes them very useful in the non-destructive evaluation of reinforced concrete member, as they provide the early warnings for the initiation of cracking.
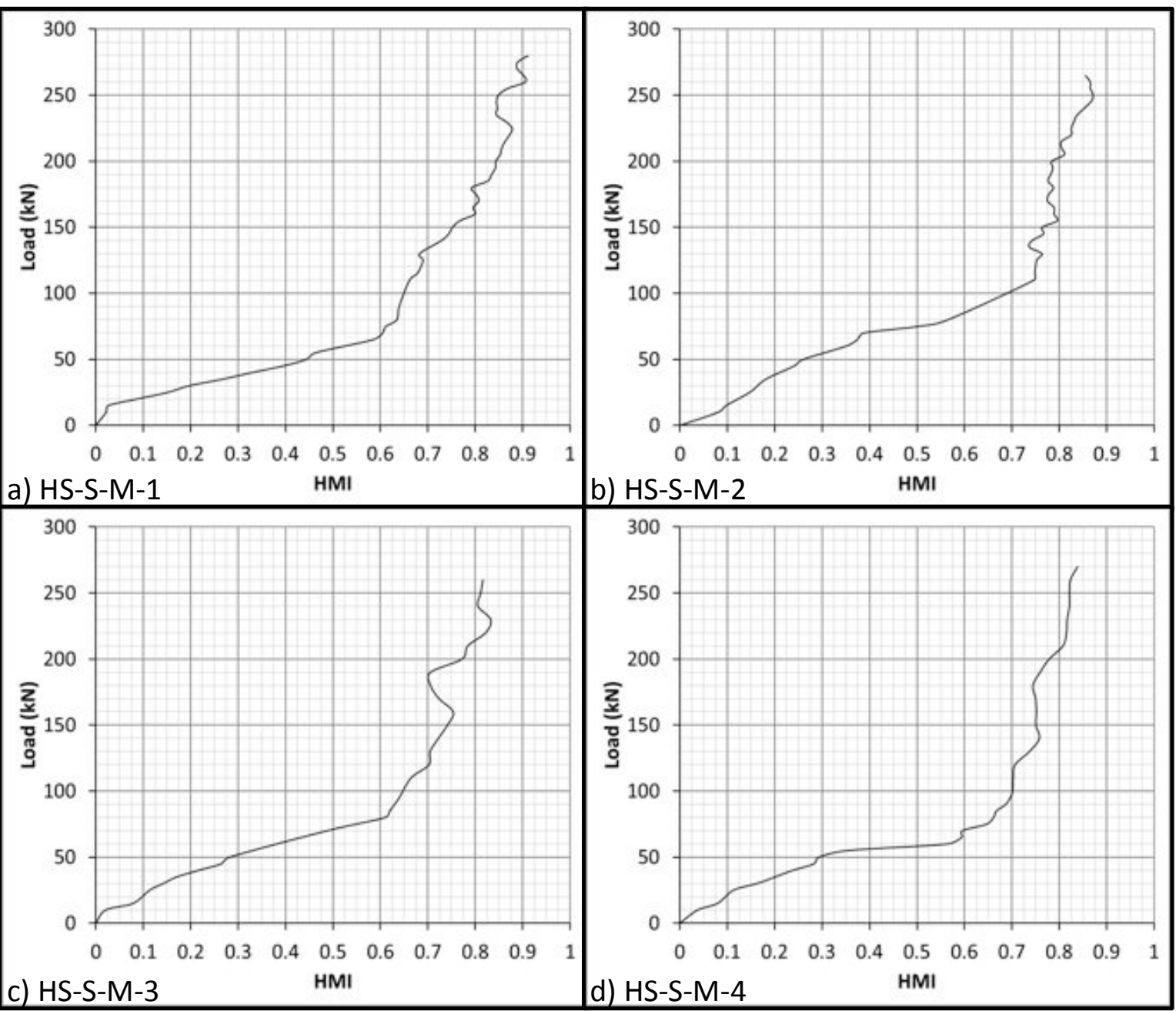

Figure 6.6: Load-HMI Curves, Small Scale Monotonic Loaded Members; (a)HS-S-M-1; (b) HS-S-M-2; (c) HS-S-M-3; (d) HS-S-M-4 
Beam HS-S-M-2 was found to have a $50 \mathrm{kN}$ cracking load, indicated by the crack gauges, and $65 \mathrm{kN}$ observed the first cracking load. The corresponding HMI values at these loads are: 0.26 at $50 \mathrm{kN}$, and 0.37 at $65 \mathrm{kN}$. Similarly beam HS-S-M-3 cracked at a load of $50 \mathrm{kN}$ as proposed by the crack gauges, and $70 \mathrm{kN}$ was the observed cracking load. The HMI values, at the respective loads, were: 0.28 at $50 \mathrm{kN}$, and 0.48 at $70 \mathrm{kN}$. Beam HS-S-M-4 had an HMI value of 0.29 at the cracking load of $55 \mathrm{kN}$, and 0.56 at the observed cracking load of $60 \mathrm{kN}$. Therefore, the test results would suggest that a member exceeding an HMI value of 0.26 could be considered to have initiated internal cracking, and at an $\mathrm{HMI}$ exceeding 0.37 the cracking would be visible on the exterior of the member.

The suggested limits are not the only indication of cracking within the members, it is also apparent that the rate of increase of the HMI is largely affected in all four beams. For beam HS-S-M-2 the rate of change of the HMI is calculated to be 0.0052 (HMI/Load) up to the cracking load of $50 \mathrm{kN}$; after cracking the rate of change increases to 0.0072 up to the observed cracking load. This large increase in the rate of change of the HMI is very indicative of the appearance of cracking through the signal path. Beam HS-S-M-3 had a rate of change of 0.0056 up to the cracking load, and 0.01 up to the observed cracking load, and beam HS-S-M-4 demonstrates an increase in the rate of change from 0.0058 to 0.027 . This large increase in the rate of change is found to be very consistent throughout the experimental data and is very important in detecting the development of cracking. Although beam HS-S-M-1 proved to be an outlier in this case, where it actually experienced a decrease in the rate of change from 0.0088 to 0.0072 , the beam also demonstrated the highest values of $\mathrm{HMI}$ at the two monitored cracking loads (crack gauge indicated and observed), and thus would have provided a clear warning of the appearance of cracking.

The proposed non-destructive system is primarily intended for the prediction, detection, and quantification of cracking within a reinforced concrete member. The results obtained from the small scale monotonic members confirm the effectiveness of this system for predictive health monitoring, as well as its ability to detect cracking within the concrete matrix. By adopting the limits for HMI discussed above, the system would be a useful tool for providing maintenance personal with information as to how close a member is to 
cracking, at critical section that could cause a member failure. Moreover, the HMI limits will let engineers know exactly the time a crack initiated as well as its location within the concrete member.

\subsubsection{HMI-Strain Relationship Monotonic Beams}

In order to use the piezo-sensor system to quantify the development of cracking within the concrete matrix, a relationship between the crack gauges results and the piezo-sensors needed to be realized. The HMI was related to the concrete strain as presented in Figure 6.7. The results obtained from the crack gauges, Figure 6.7a, and the piezo-sensors, Figure 6.7b, were combined over the load axis to obtain the relationship illustrated in Figure 6.7c. The graph in Figure 6.7c illustrates the relationship between the HMI values from each beam ( $y$-axis), and the strain determined by the crack gauges ( $x$-axis). The relationship is important, as it will provide the basis for the crack width approximation model developed in the current study.

The four beams are plotted in Figure 6.7c, as to show the trend between the results obtained from each specimen. The four specimens seem to provide similar results, showing a clear logarithmic trend for the HMI-Concrete Strain relationship. Members HS-S-M-2, HSS-M-3, and HS-S-M-4, show very consistent results between one another up to a concrete strain of $2500 \mu$-strain. Member HS-S-M-1 is found to follow the same trend as the other three beams up to $2000 \mu$-strain. The average relationship between the $\mathrm{HMI}$ and concrete strain is also shown in Figure 6.7c. The results of the four specimens were averaged as to give one relationship for the $\mathrm{HMI}$ and concrete strain that summarized the results of the monotonically loaded members. The average HMI-Strain relationship could be analyzed, and used for the development of the desired HMI-Crack Width model. Due to a slight variation noticed between the cracked and un-cracked specimens behavior, the model is separated into two distinct sections. This is achieved by limiting the values of strain to a maximum reading of $110 \mu$-strain. Equation 6.2 approximates the average concrete strain between two piezo-sensor for both the pre and post-cracking stages of the concrete section. 


$$
H M I=\left\{\begin{array}{rrr}
0.1217 \ln (\varepsilon)-0.2664 ; & 0 \leq \varepsilon \leq 110 \\
0.1335 \ln (\varepsilon)-0.2875 ; & 110 \leq \varepsilon \leq 3500
\end{array}\right.
$$
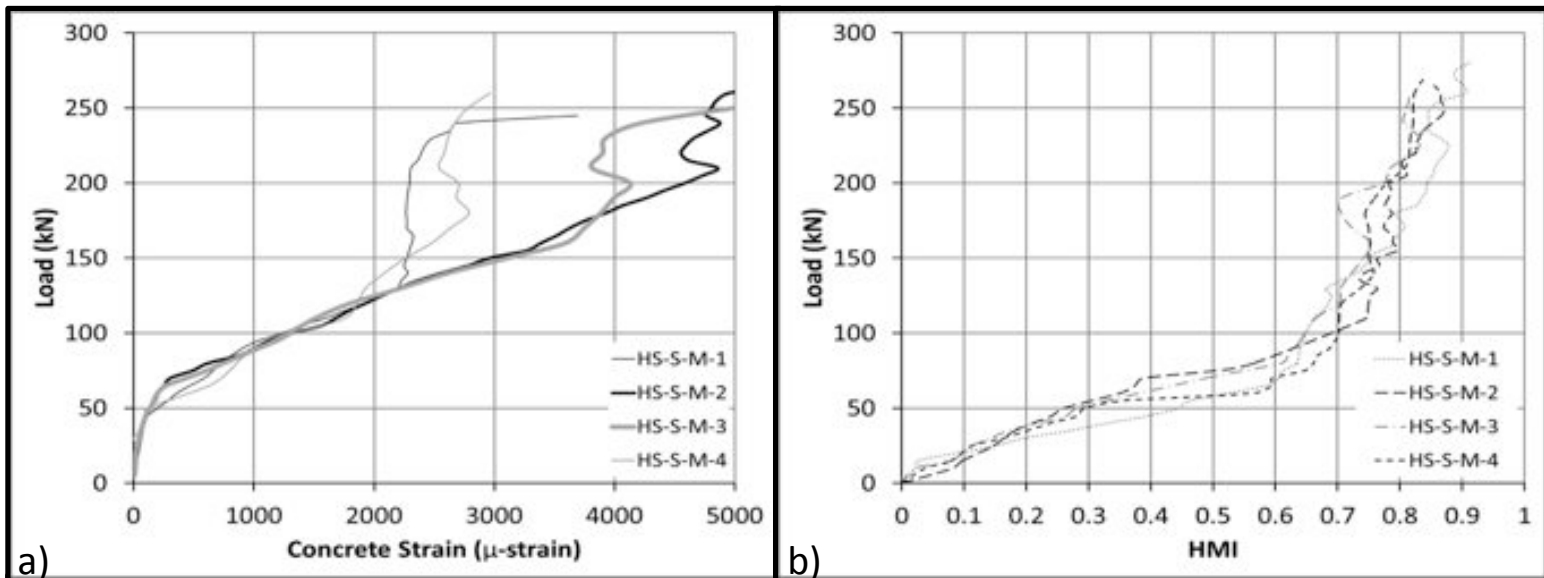

a b)

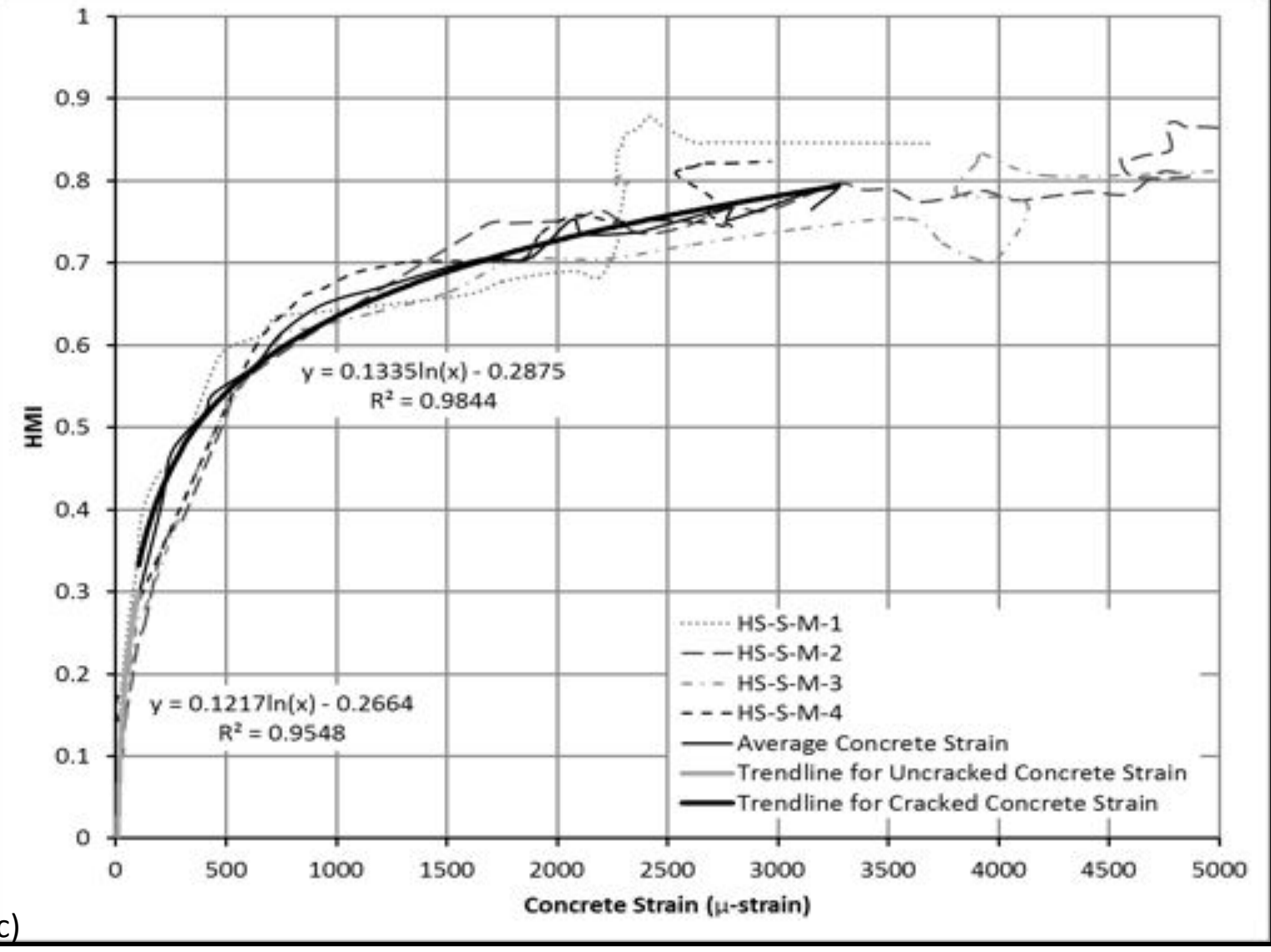

Figure 6.7: Summary Of Small-Scale Results; (a) Load-Strain Reading, Crack Gauges; (b) Load-HMI Readings (c) Average HMI- Crack Width Relationship 


\subsection{Small Scale Beams under Cyclic Loading (HS-S-C)}

The HS-S-C experimental program consisted of three small-scale beams that were tested using cyclic loading routines, and carries the designation "HS-S-C-\#". The beams were equipped similar to the monotonic members, although each beam had it's own specific subroutine for the loading procedure. The load in each member was varied in load steps of $5 \mathrm{kN}$ throughout the duration of the test, and the results of the experimental testing is presented herein. From the results obtained, a relationship between the concrete strain and $\mathrm{HMI}$ values is developed, that is later used to establish the HMI-Crack Width model. The visually observed cracking loads will also be discussed for comparison.

\subsubsection{Member Behavior}

The behaviors of the three cyclically loaded beams are presented in Figure 6.8. The loading procedure is changed for each reinforced concrete beam. Member HS-S-C-1 is exposed to a single cycle at three different load levels: 50 kN, 100 kN, and 150 kN; beam HS-S-C-2 and HSS-C-3 both undergo cycles at loads of: 50 kN, 70 kN, 100 kN, 150 kN and 200 kN, where beam HS-S-C-2 is subjected to two cycles increments at each load, and beam HS-S-C-3 is exposed to three cycles. All three beams were designed to be identical in terms of: geometry, reinforcement detail, and material properties; differing only by the loading procedure.

The Load-Deflection graph for member HS-S-C-1 is presented in Figure 6.8a. The three distinct loading cycles can be seen to have occurred at the specified load of $50 \mathrm{kN}, 100 \mathrm{kN}$, and $150 \mathrm{kN}$, followed by the member being loaded up to its failure load of $265 \mathrm{kN}$ and reaching an ultimate deflection of $5.15 \mathrm{~mm}$. Member HS-S-C-2 is shown in Figure 6.8b. The member behavior is found to be similar to beam HS-S-C-1, although five distinct loading cycles were employed. The coinciding loading steps between beams HS-S-C-1 and HS-S-C-2 are seen to exhibit similar results. Where the deflection of member HS-S-M-1 is $1.22 \mathrm{~mm}$ after the $50 \mathrm{kN}$ loading cycle, the deflection of member HS-S-M-2 is slightly higher value of $1.33 \mathrm{~mm}$. 


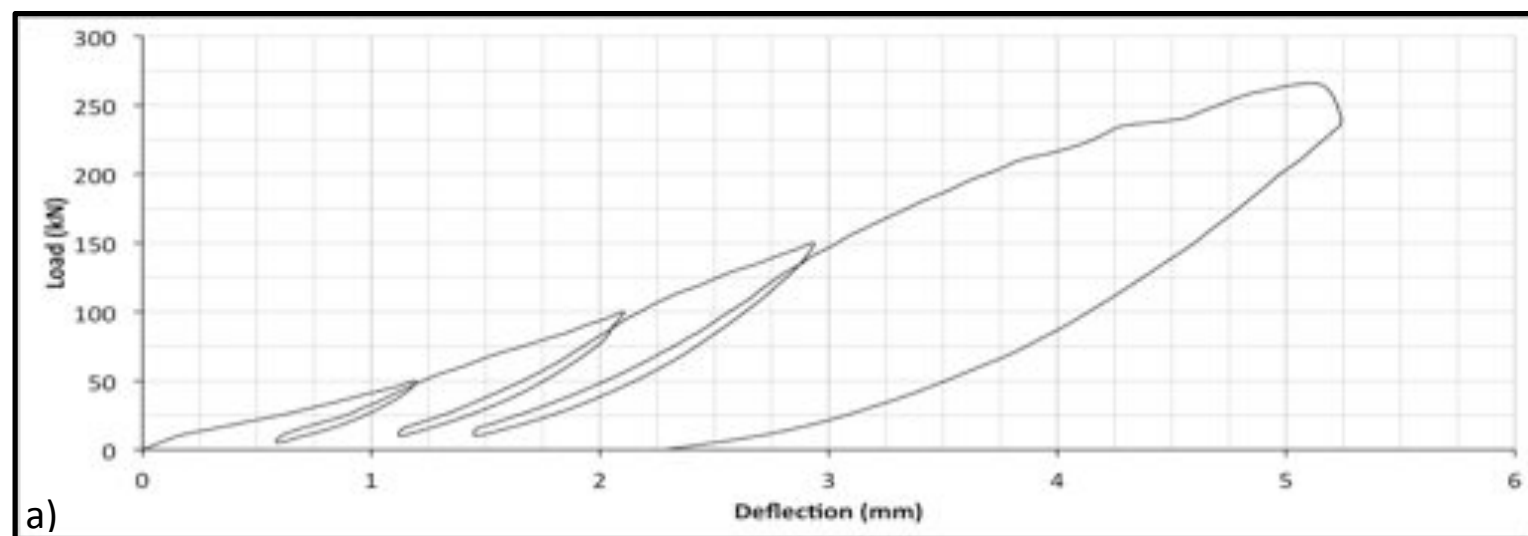

a)

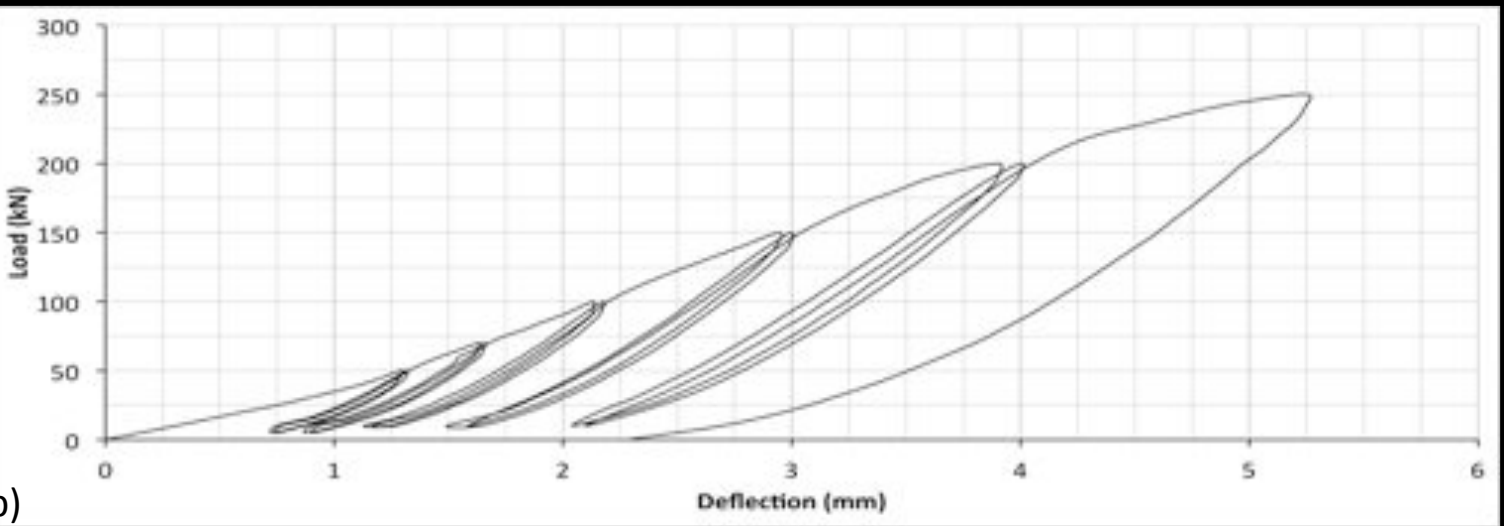

b)

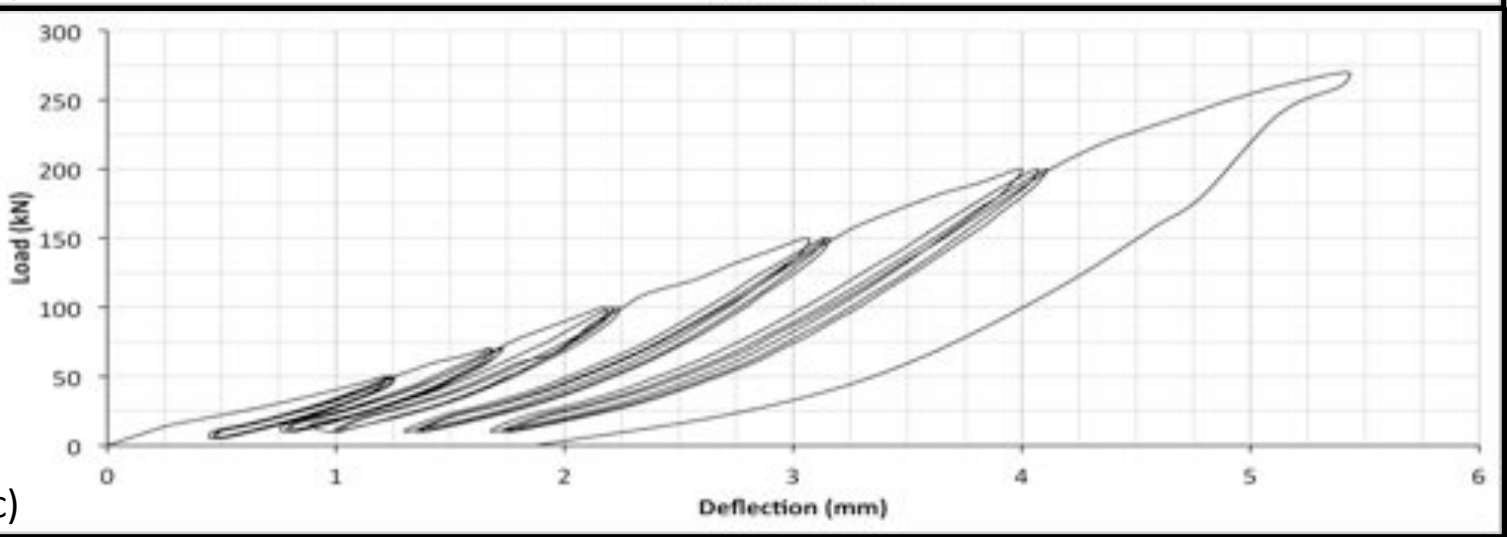

Figure 6.8: Load-Deflection Graph Small Scale Cyclically Loaded Members; (a) HS-S-C-1; (b) HS-S-C-2; (c) HS-S-C-3

Similarly, after the cycles at the $100 \mathrm{kN}$ load step, beam HS-S-C-1 showed a deflection of $2.17 \mathrm{~mm}$ where HS-S-C-2 had a deflection of $2.19 \mathrm{~mm}$. At the $150 \mathrm{kN}$ load step the deflection experienced by beam HS-S-C-1 was $3.04 \mathrm{~mm}$ and beams HS-S-C-2 demonstrated a deflection of $3.04 \mathrm{~mm}$. The final failure load of member HS-S-C-2 is $250 \mathrm{kN}$ at an ultimate deflection of $5.25 \mathrm{~mm}$. Overall, the alternative loading procedure between members HS-S-C-1 and HS-S-C-2, showed slight variations in the behavior of each member, where the 
more cycles that the member was exposed to, the higher the value of deflection at any specific load step.

The load deflection graph for member HS-S-C-3 is presented in Figure 6.8c. The five distinct load steps undergoing cyclic loading are easily observed, along with the three-cycle repetition. The behavior of the member is found to be similar to that of beam HS-S-C-2. As beam HS-S-C-3 underwent three repetitions for each load step exposed to cyclic loading, the deflection is expected to be slightly higher than that of beam HS-S-C-2 after any given load step. The deflection at the $50 \mathrm{kN}$ load cycles for member HS-S-C-2 is $1.33 \mathrm{~mm}$ where in beam HS-S-C-3 it was found to be $1.25 \mathrm{~mm}$. After the $70 \mathrm{kN}$ cyclic load step the deflection was found to be $1.68 \mathrm{~mm}, 1,73 \mathrm{~mm}$ for beam HS-S-C-2 and HS-S-C-3, respectively. After the $100 \mathrm{kN}$ load step the deflection of the beam HS-S-C-2 has a deflection of $2.18 \mathrm{~mm}$, and beam HS-S-C-3 has a deflection of $2.24 \mathrm{~mm}$. This trend was consistent for the $150 \mathrm{kN}$ load step and $200 \mathrm{kN}$ load steps, demonstrating that the results should be very comparable between the three cyclic beams, and will be reflected in the results obtained from the piezo-sensors, and crack gauges. The failure load of member HS-S-C-3 was recorded at 270 $\mathrm{kN}$ and an ultimate deflection of $5.42 \mathrm{~mm}$.

\subsubsection{Crack Gauge Readings}

Like the monotonic beams, the cyclic beams were equipped with crack gauges for measuring the strain at the center location of the beam. A crack was induced as described in the experimental program. Each beam was equipped with two crack gauges, one on either face. The results of the two gauges were averaged, as to give a measure of the crack width at the center of the beam. The results obtained from the crack gauges for the three cyclic beams are presented in Figure 6.9. These gauges were anchor bolted to each face of the beams. The results obtained show that the behavior of beam HS-S-C-1 is very similar to that

of beam HS-S-C-2, as illustrated in the Load-Deflection graphs, and HS-S-C-1 proves to be slightly stiffer in its behavior. Beam HS-S-C-3 experienced the highest number of loading cycles, although showed a very stiff behavior in terms of cracking. 


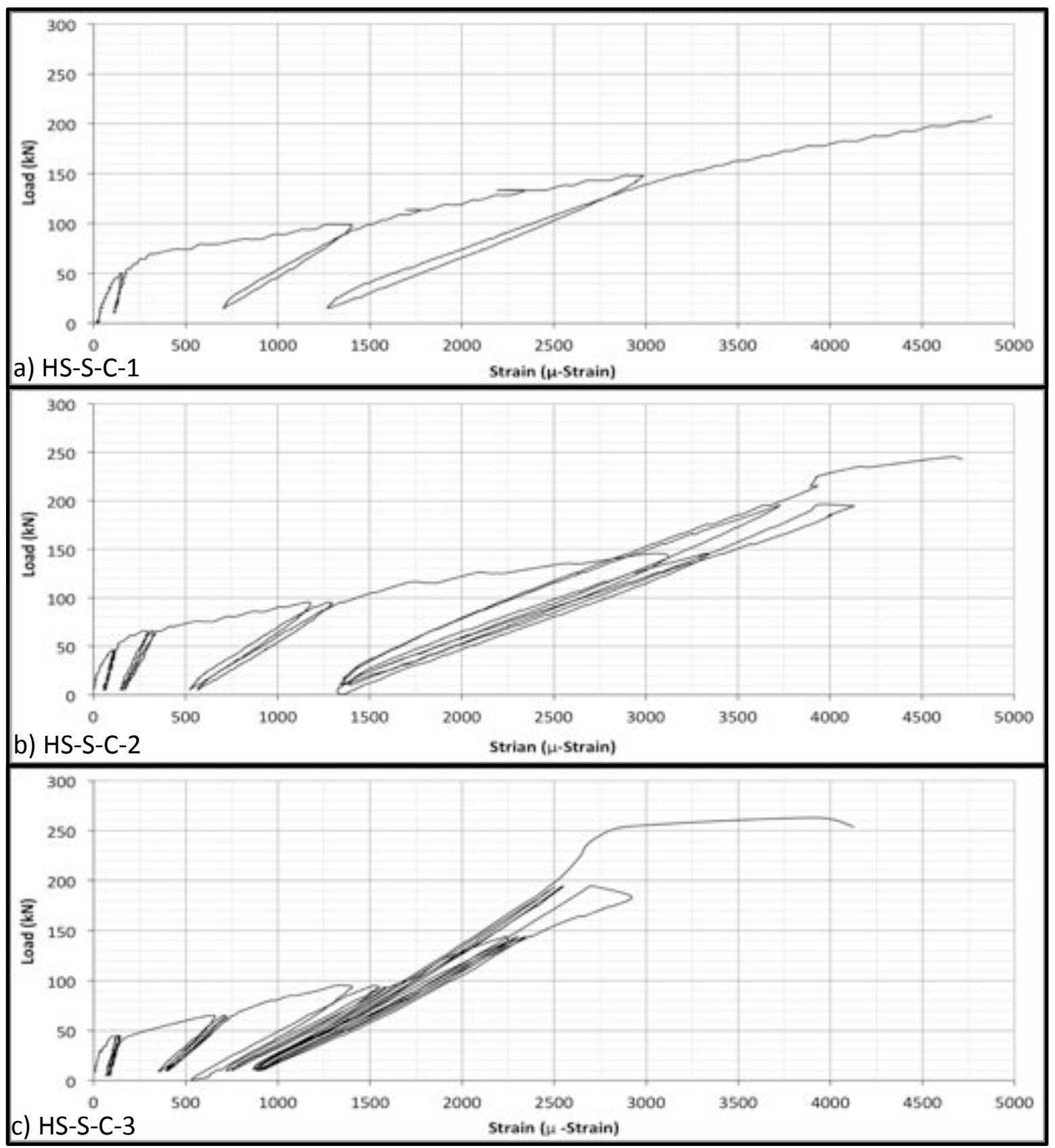

Figure 6.9: Load-Strain Reading from Crack Gauges, Small Scale Cyclically Loaded Members; (a) HS-S-C-1; (b) HS-S-C-2; (c) HS-S-C-3

It should be noted that during the testing of the three cyclic specimens there was a slight variation to the intended experimental program. The experimental program for beam HS-SC-1 was carried out in one continuous test, whereas the experimental work for beam HS-SC-2 and HS-S-C-3 was interrupted due to time constraints. The experimental work for both 
beams HS-S-C-2 and HS-S-C-3 were interrupted after the completion of the $150 \mathrm{kN}$ loading cycles, for a period of 12 hours, in which the beam may have undergone some relaxation.

The results obtained from the three beams have some similarities for the propagation of cracking in the three members. The strain is seen to increase with the increase in load, and decrease with the release, as expected, up to a certain threshold where the increase in load will not have a major effect on the opening of the induced crack, and the cycles seem to fall one on top of the other. This is evident from beams HS-S-C-2 and HS-S-C-3, where the 50 $\mathrm{kN}, 70 \mathrm{kN}$ and $100 \mathrm{kN}$ step cycles are clearly differentiable. Although the load cycles occurring at the $200 \mathrm{kN}$ and $250 \mathrm{kN}$ load steps fall in very close range to one another, showing that the crack will open and close linearly with the increase and decrease in load. This could be due to the interruption in the loading procedure of the beam, as the reinforcement had time to relax, and thus the measured crack would have had time to

\subsubsection{Piezo-Sensor Results}

The piezoceramic patches in the cyclic experimental program were installed just as in the monotonic beams, and monitored the appearance of a crack across the centerline of the beam. The results are expected to provide a direct comparison between the crack gauges and piezo-sensors, and would verify if the piezo-sensors are sensitive to the reduction in crack width, or will always yield the maximum damage inflicted in the member. The Load$\mathrm{HMI}$ values obtained from the piezo-sensors, for each individual beam is presented in Figure 6.10 .

Beam HS-S-C-1, in Figure 6.10a, shows a significant reduction in the HMI readings for the 50 $\mathrm{kN}$ cycle. The HMI leading up to the $50 \mathrm{kN}$ load is seen to increase almost linearly to a value of 0.26 . As the load was released, the $\mathrm{HMI}$ value decreases to a value of 0.24 at a load of 5 $\mathrm{kN}$. This behavior is consistent with members HS-S-C-2 in Figure 6.10b, and HS-S-C-3 in Figure 6.10b. Beam HS-S-C-2 is seen to have an increasing HMI value up to the $50 \mathrm{kN}$ load step, reaching a value of 0.28 . As the load in beam HS-S-C-2 is released, the HMI value is seen to decrease as well to a value of 0.24 . The beam is then loaded back to $50 \mathrm{kN}$ and the 
$\mathrm{HMI}$ value raises to 0.32 , from where the release in load brought the $\mathrm{HMI}$ value back to 0.27. The beam was then loaded back to $50 \mathrm{kN}$ where the HMI value was found to be 0.36 .

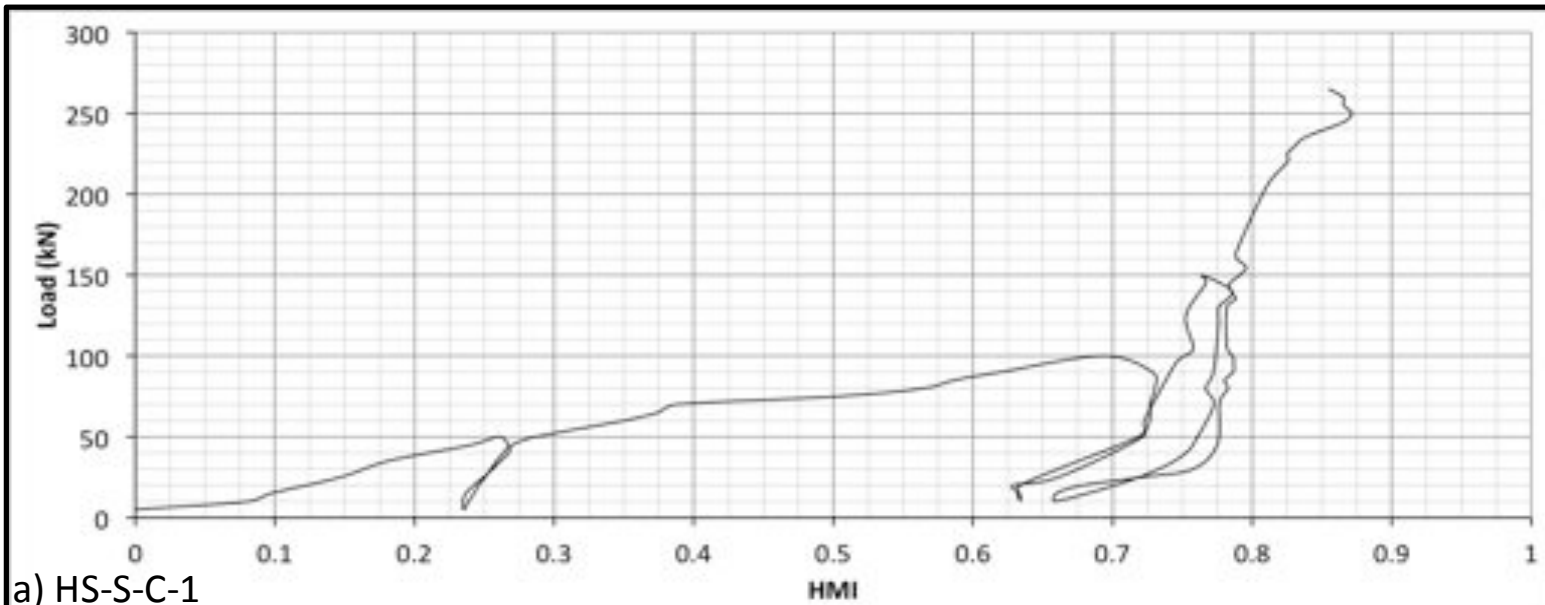

a) HS-S-C-1

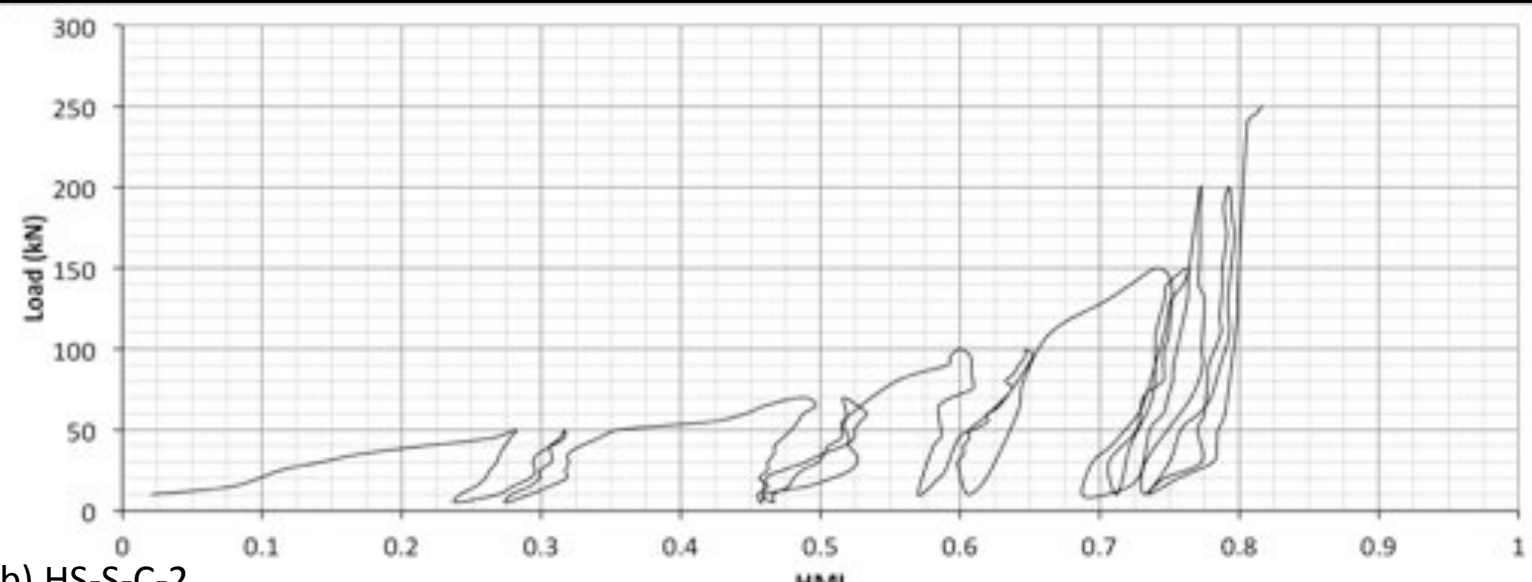

b) HS-S-C-2 HM

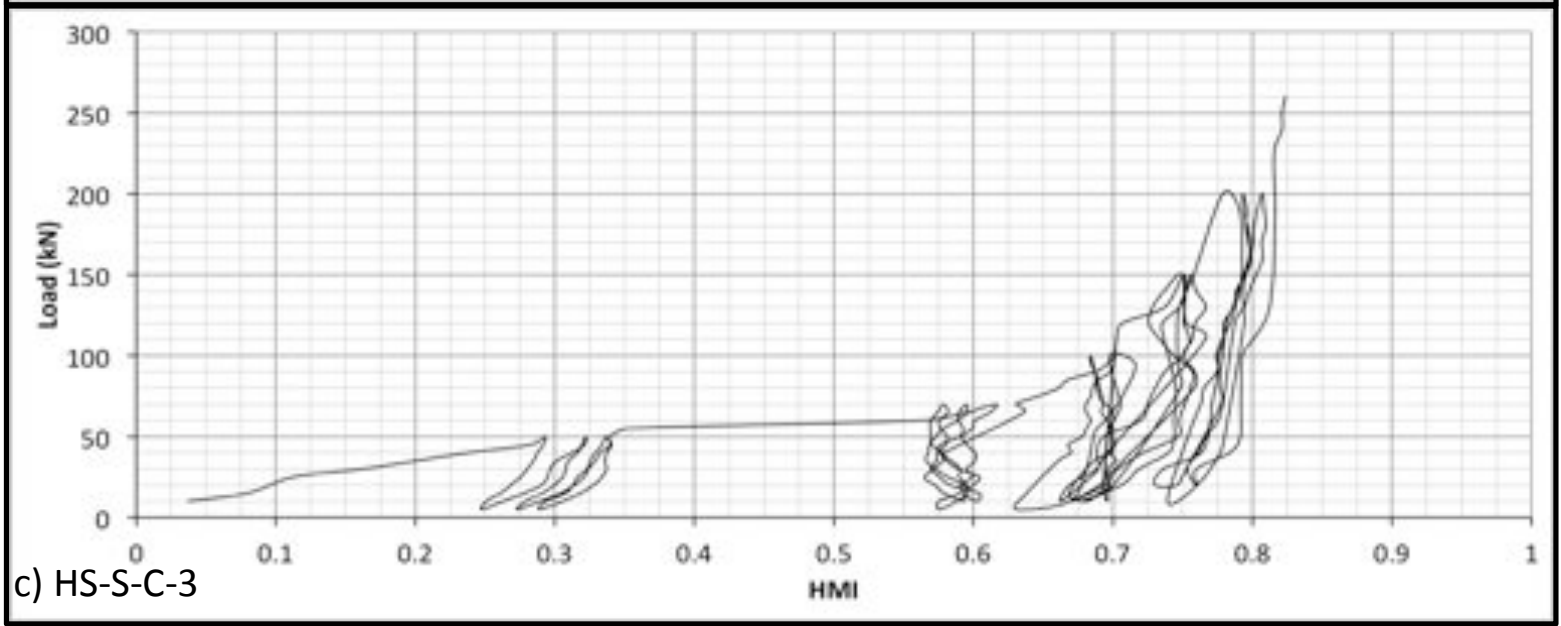

Figure 6.10: Load-HMI Curves, Small Scale Cyclically Loaded Members; (a) HS-S-C-1; (b) HS-S-C-2; (c) HS-S-C-3 
A similar behavior is seen in Figure 6.10c for beam HS-S-C-3. The HMI value for the beam increases to 0.29 at $50 \mathrm{kN}$; then decreases to 0.25 when the load is released; increases to 0.32 after the first cycle is completed; then decreases to 0.27 when the load is released again; increases to 0.34 upon the completion of the second cycle; the HMI value decreases to 0.29 when the load is released again, and increases to 0.34 upon the completion of the $50 \mathrm{kN}$ load cycles. The consistency between the various loading programs of the three specimens would suggest that the piezo-sensors react sufficiently to the release in internal strain of the beams, although this is not the case for the later loading cycles.

From the experimental results obtained it can be seen that consistently throughout the results of the three cyclic members, the HMI is visibly affected at the first set of cycles (0-50 $\mathrm{kN})$, and is very marginally affected by the release of load in the later loading cycles. This is thought to be an effect of the crack width opening through the signals path. Prior to the appearance of cracking all three beams follow the expected behavior where the increase in load will increase the $\mathrm{HMI}$ value, and the decrease in load will decrease the HMI value, although once the member cracks this is not the case.

Beam HS-S-C-1 was found to exceed the cracking strain, of $110 \mu$-strain, during the completion of the loading cycle as it reached the load of $40 \mathrm{kN}$ for the second time, and had an $\mathrm{HMI}$ value of 0.27 . Beam HS-S-C-2 exceeded the tensile cracking strain at a load of after the completion of the second load cycle, at a load of $50 \mathrm{kN}$, and had an $\mathrm{HMI}$ value of 0.36 . Beam HS-S-C-3 was found to crack after the completion of the first loading cycle at a load of $50 \mathrm{kN}$, having an $\mathrm{HMI}$ value of 0.32 .

\subsubsection{HMI-Strain Relationship Cyclic Beams}

The analysis carried out in the monotonic small-scale experimental program, was repeated for the cyclically loaded beams. The analysis helps provide a link between the piezo-sensors and the crack gauge readings. The HMI-Concrete Strain relationship obtained from the cyclic experimental program is compared to the model developed from the monotonically 
loaded beams to develop the general HMI-Crack Width, and HMI-Concrete Strain model that can be applied to the investigation carried out in the full scale experimental program.

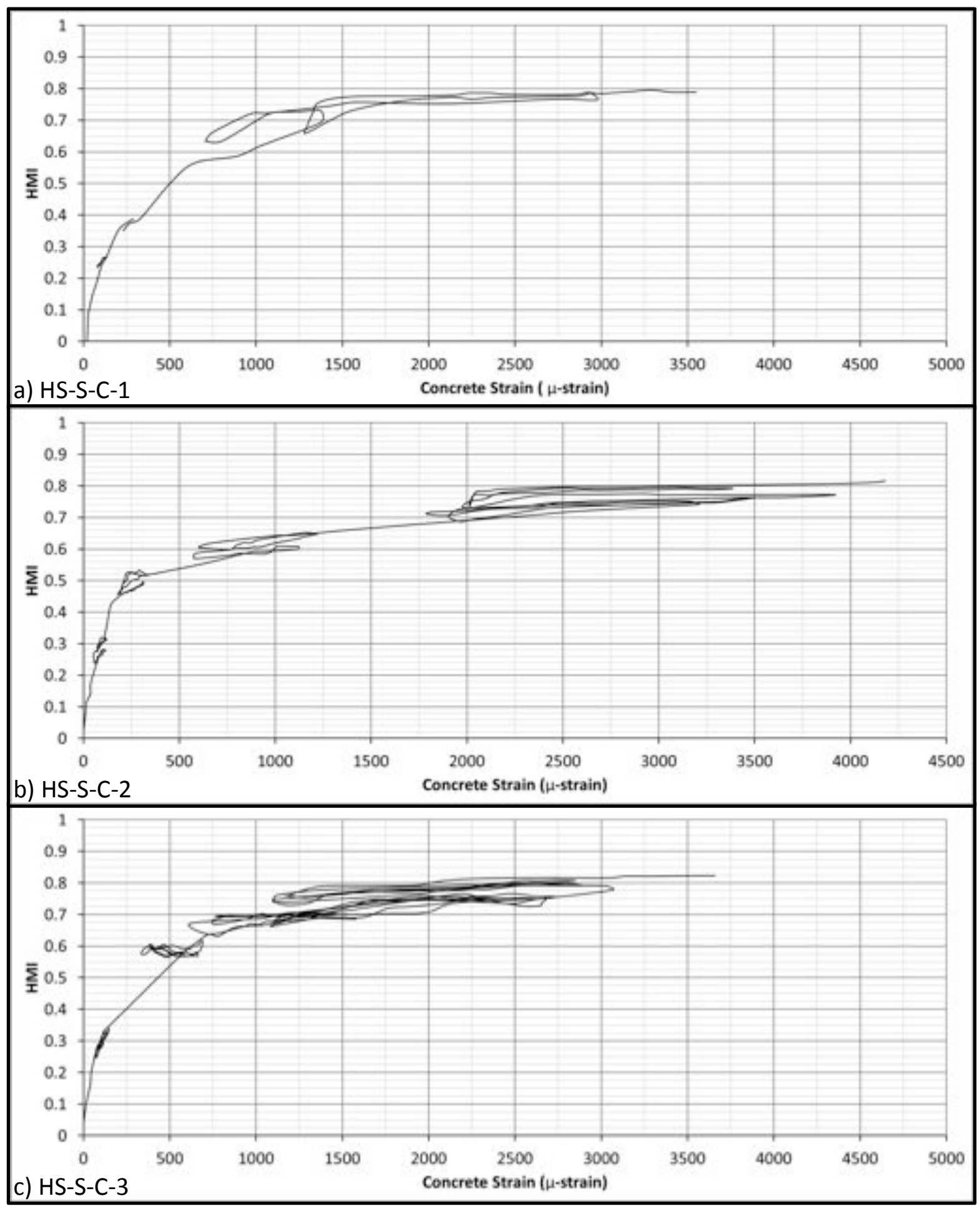

Figure 6.11: HMI-Strain Relationship of Small Scale Cyclically Loaded Beams; (a) HS-S-C-1; (b) HS-S-C-2; (c) HS-S-C-3 
The HMI-Concrete Strain relationships for the three cyclic beams are presented in Figure 6.11. Had the piezo-sensors been able to measure the crack width both during the increase in load and the release stages, the cycles of the three beams would be indistinguishable on the HMI-Concrete Strain graphs, as they would follow the logarithmic curve. The results would look like the $50 \mathrm{kN}$ cycle presented on each on the three graphs. These cycles seem to blend in the general trend line and are nearly indistinguishable. This trend shows that the piezo-sensors demonstrate a relationship that is similar to the strain readings provided by the crack gauges. It can be clearly seen that the experimental data obtained from the later cycles does not represent the physical behavior accurately. This is noticed by the lack of correlation between the $\mathrm{HMI}$ values and the physical concrete strain.

As the $50 \mathrm{kN}$ loading cycles are at the limit of cracking for the three members, the results for this load cycles can be considered to be the un-cracked tensile concrete strain of each beam, where loads exceeding $50 \mathrm{kN}$ are representative of the cracked concrete beams. Thus, the piezo-sensors are useful for measuring the tensile concrete strain prior to cracking; although once the member is cracked the sensors represent the maximum inflicted damage, as suggested by the lack of change in the HMI value as the load in the member is released.

The three beams are put through cycles at the $100 \mathrm{kN}, 150 \mathrm{kN}$, and $200 \mathrm{kN}$ load steps. The $\mathrm{HMI}$ value is seen to be almost the same throughout the repetitions, although will shift upwards for every cycle completion, representing the increase of the maximum crack width of the members. Thus, the sensors are effective in capturing the maximum induced strain over the life of a member and could be considered beneficial in the qualification of damage induced by random loads. The $50 \mathrm{kN}$ load cycles are the portion of the HMI-Concrete Strain curves between the $\mathrm{HMI}$ values of 0.25 and 0.35 . Thus in order to properly interpret the data presented by this experimental program, and provide an accurate HMI-Concrete Strain relationship, the cycles after the initiation of cracking in the member should be disregarded, and should be interpreted as monotonic data from zero load to the failure load.

As carried out in the experimental data of the monotonic beams, the concrete strain is plotted on the x-axis, and HMI on the y-axis, as shown in Figure 6.12. The average 
HMI-Concrete Strain is plotted and the two trend lines are deduced to represent the member's behavior prior to cracking and post-cracking. The same logarithmic trend is observed as in the monotonic beam data, although the equations of the HMI-Concrete Strain relationship differ slightly. The data obtained from beams HS-S-C-1 and HS-S-C-3 are seen to be very consistent. The two members follow almost the same curve. Member HS-SC-2 yielded slightly different results, and is the outlier for the experimental data. The average curve of the three specimens are presented in Figure 6.12, along with the trend curves used to obtain the HMI-Concrete Strain relationships, both prior to cracking and after cracking. Equation 6.3 represents the concrete strain of the monitored section based on the results of the cyclic tests.

$$
H M I=\left\{\begin{array}{cr}
0.1007 \ln (\varepsilon)-0.1828 \\
0.136 \ln (\varepsilon)-0.2962 \quad ; \quad 110 \leq \varepsilon \leq 3500
\end{array}\right.
$$

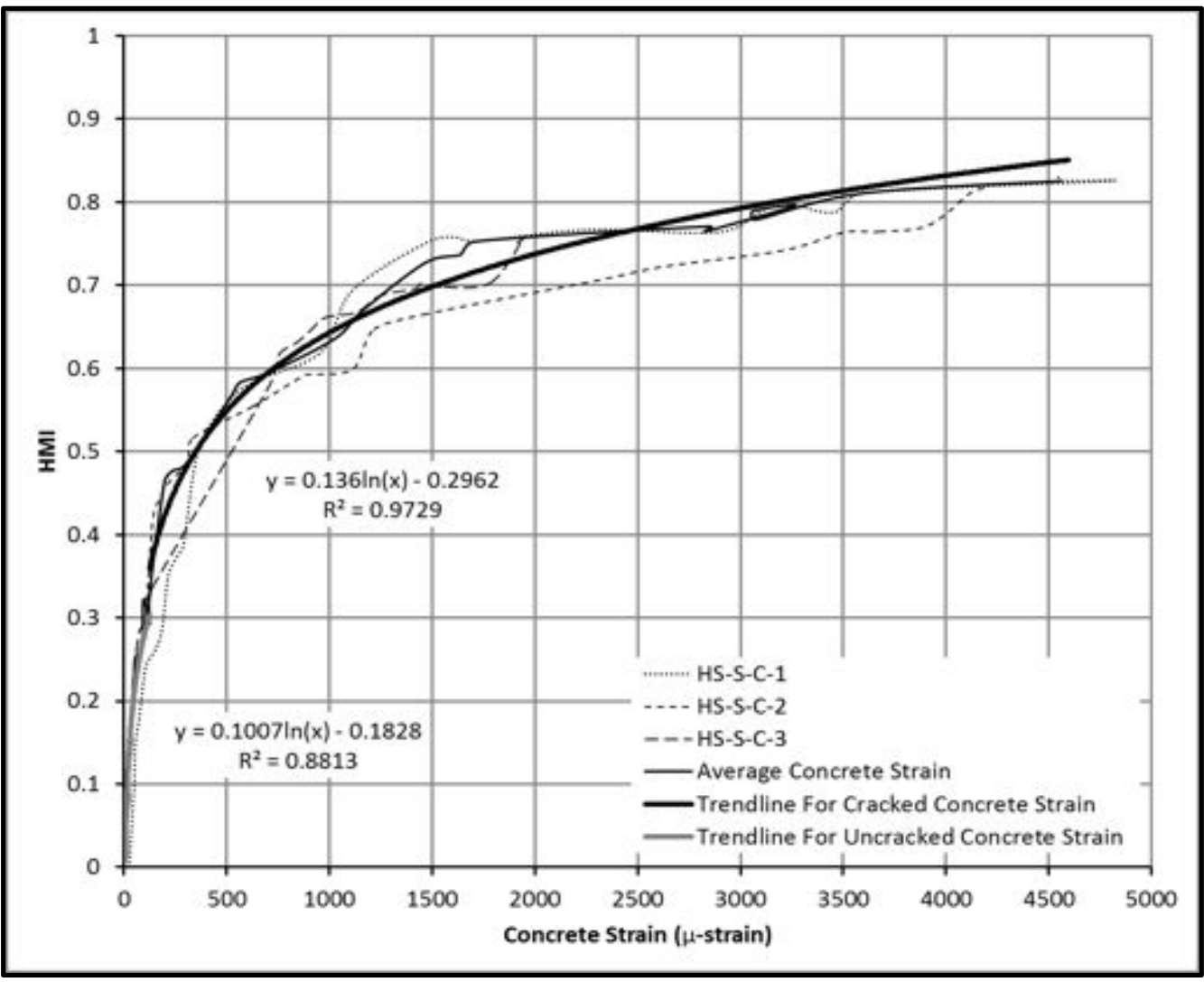

Figure 6.12: Average HMI-Crack Width Relationship as Suggested by Small Scale Cyclically Loaded Beams 
The equations obtained from the monotonic and cyclic small-scale experimental data will be compared and contrasted in the following section, as to provide a single general HMI-Crack Width model for the piezo-sensors, based on the results of both experimental programs. The model will be developed based on the trends observed from the two small-scale experimental programs (monotonic and cyclic).

\subsection{Development and Verification of Crack Width-HMI Relationship}

The experimental work provided two mutually exclusive models for predicting the crack width from the results of $\mathrm{HMI}$, the monotonic model (HS-S-M), represented by Equation 6.2 and the cyclic model (HS-S-C), represented by Equation 6.3. To simplify the use of the developed models, one general model to express the Crack Width-HMI value is developed; especially a high similarity between the two models is obtained. The following section compares the direct crack widths obtained from the crack gauges to the results of each crack width models to provide the best alternative for crack width estimation.

The experimental results obtained from the monotonically loaded beams are presented in Figure 6.13. The graphs present the results obtained from either crack gauge installed on each member (right and left sides), the results for crack width when processed with the equation obtained from the monotonically loaded members, and the results obtained when processing the HMIs using the equation suggested by the cyclically loaded small-scale beams. A comparison is also drawn between the average measured crack width of each beam, and the recorded results obtained by each crack width models, as to establish the best alternative for crack-width estimation. The comparison between the proposed alternatives and the measured crack widths can be realized in terms of the standard deviation between the average measured crack width, and the results yielded by the two HMI-Strain models. The general model is chosen based on the lowest average standard deviation of the two HMI-Crack Width models.

The same investigation is carried out using the data obtained from the cyclically loaded members, and the analysis is repeated. The results from the cyclically loaded beams are 
presented in Figure 6.14. A single HMI-Crack Width equation will be adopted for the analysis of the large-scale beams. The equation will be selected based on its comprehensive performance for all seven small scale specimens.

\subsubsection{HMI-Crack Width Models}

The experimental work provided two mutually exclusive models for relating the strain experienced by a member to the results obtained from the piezo-sensors. The strain provided from the reading obtained by the crack gauges, was related to the HMI reading provided by the piezo-sensors as to develop a relationship that could lead to the prediction of the crack width in a reinforced concrete member from the signal output obtained by the proposed piezo-ceramic system. The HMI-Strain relationships obtained from the experimental work can be converted to the desired HMI-Crack Width relationships using the effective gauge length of the crack gauges, after subtracting the value of the average concrete cracking strain (110 $\mu$-strain). By rearranging Equations 6.2 and 6.3, introducing the effective crack gauge length and the cracking strain limit, the desired HMI-Crack Width relationships can be obtained. The HMI-Crack Width equation obtained from the monotonically loaded small scale beams is presented in Equation 6.4, and the equation obtained from the cyclically loaded members is presented in Equation 6.5. Where c.w. is the crack width, $\mathrm{HMI}$ is the Health Monitoring Index at any specific time through the member's life, and 110 term represents the average cracking concrete strain for a reinforced concrete sample.
(Monotonic Equation)
$C . W .=\frac{\left(e^{\frac{H M I+0.2875}{0.1335}}-110\right)}{10000}$
(Cyclic Equation)

$$
\text { C. W. }=\frac{\left(e^{\frac{H M I+0.2962}{0.136}}-110\right)}{10000}
$$

In order to develop a general HMI-Crack Width model, Equation 6.4 and 6.5 are used to back calculate the crack width of each of the seven small-scale samples. The crack width 
obtained using these equations were compared to the average measured crack width provided by the crack gauges, as to determine the effectiveness of each model. The HMICrack Width relationship that provides the best results was adopted in the analysis of the large-scale specimens, and investigates the piezo-systems application as a full-scale comprehensive non-destructive technique.

\subsubsection{Verification of Crack Width-HMI Relationship Based on Results Obtained from the Monotonically Loaded Small Scale Beams}

The results of the direct crack width measurements, and the results obtained from the piezo-sensors for the monotonically loaded beams are presented in Figure 6.13. Here the results of beam HS-S-M-1 is shown in Figure 6.13a. The crack gauges of this beam provided accurate results of the effective crack width up to a load of $140 \mathrm{kN}$, after which the crack gauges failed, and provided no further information. Based on both suggested HMI-Crack Width models, the results provided by the piezo-sensors demonstrated consistent results up to a load of $210 \mathrm{kN}$ for the monotonic beams. The more remarkable aspect of the data obtained from beam HS-S-M-1 is the continuation of data provided by the piezo-sensors after the crack-gauges failed. The results of the piezo-sensors proved to provide similar trends illustrated by the crack gauges prior to their malfunction. Although both models yield similar results, the HS-S-M model provides a better fit to the direct crack width measurements provided by the installed crack gauges.

The results obtained from beam HS-S-M-2 are presented in Figure 6.13b. The installed crack gauges provided results for the crack width measurements up to a load of $200 \mathrm{kN}$, whereas the piezo-sensors provided readings up to the failure load of $260 \mathrm{kN}$. From the projected Load-Crack Width graph, it can be seen that the results of the two crack gauges are very similar to one another, and are consistent with the results obtained from the two crack width models of the piezo-sensors, up to a load of $150 \mathrm{kN}$. From where the cyclic model highly underestimated the internal crack width, providing misleading results. The

monotonic model provides a much more realistic approximation of the crack width, agreeing with the direct crack width readings provided by the crack gauges. 

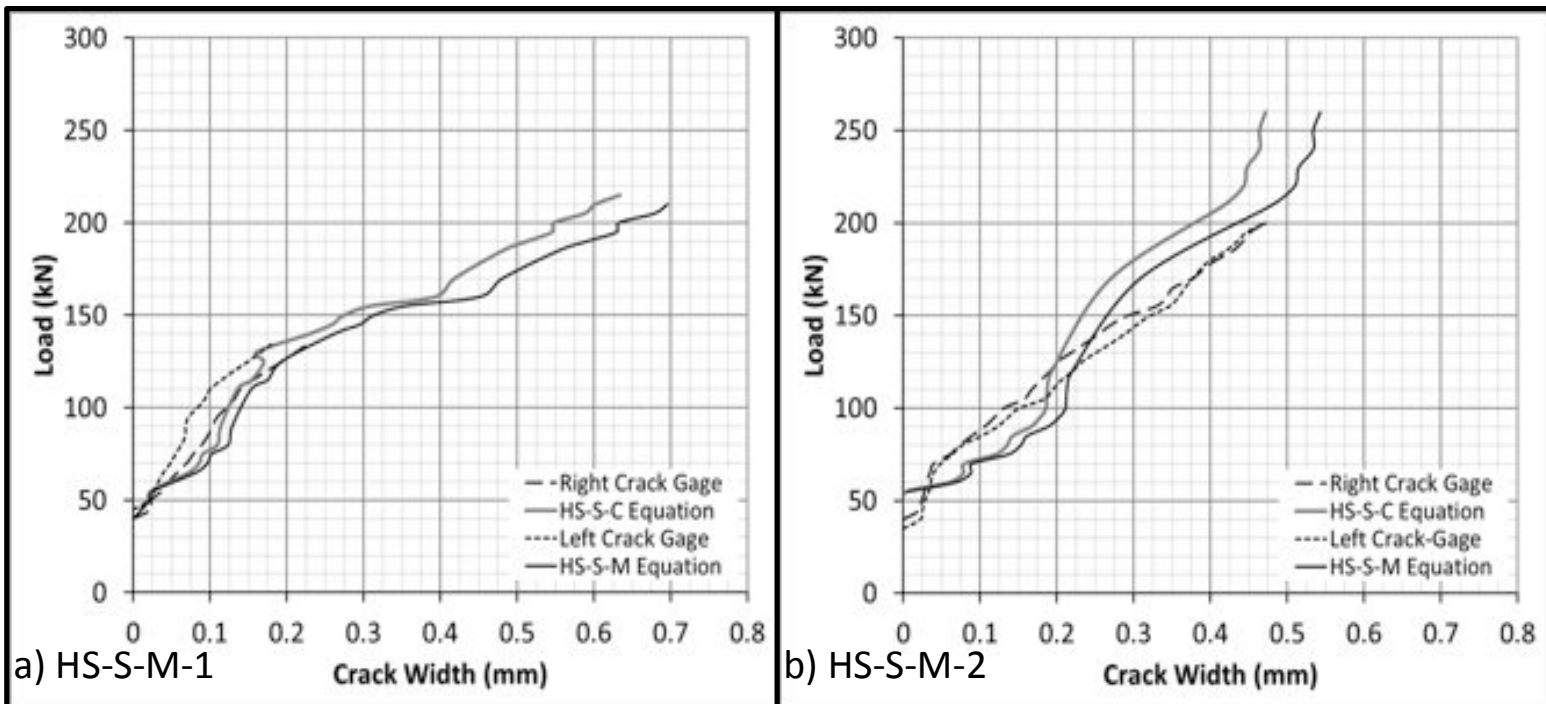

b) HS-S-M-2 Crack Width (mm)
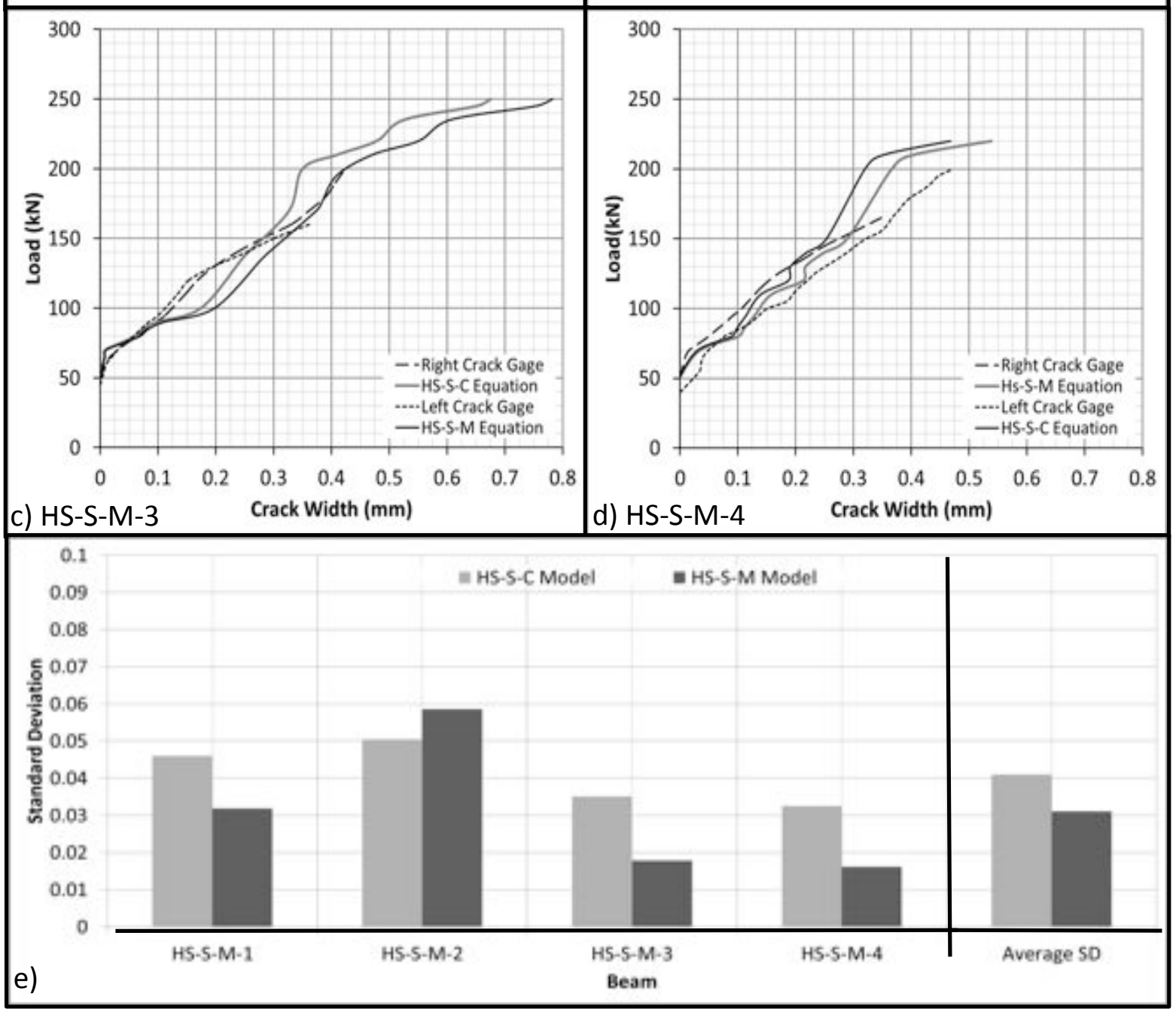

Figure 6.13: Comparison of HS-S-M and HS-S-C HMI-Crack Width Models, and Direct Crack Width Readings Using Crack Gauges, Using Experimental data from HS-S-M Beams; (a) Beam HS-S-M-1; (b) HS-SM-2; (c) HS-S-M-3; (d) HS-S-M-4; (e) Computed Standard Deviation of Cyclic and Monotonic Models 
The results obtained from beam HS-S-M-3, Figure 6.13c, and HS-S-M-4, Figure 6.13d, provided the most accurate results to sustain the comparison of the two models, as both beams demonstrated very similar results. Beam HS-S-M-3 shows consistent results for the crack width between the crack gauge and the monotonic equation, slightly overestimating the average measured crack width. Although the cyclic model is noticed to largely underestimate the crack width, and can be considered to be inadequate for the analysis. The results of beam HS-S-M-4 are presented in Figure 6.13d. The figure shows that the installed crack gauges, of this member, were effective up to a load of $160 \mathrm{kN}$ and $200 \mathrm{kN}$. The data obtained from the piezo-sensors is seen to fall in close proximity with the direct crack measurements up to the load of $150 \mathrm{kN}$, after which the monotonic model is found to be the much more effective at measuring the crack width. Briefly, between the four specimens, the equations are seen to be very consistent in providing crack width estimations, although the trivial result is discovered to be the most effective; the monotonic equations will provide the best crack width approximation for the monotonic specimens.

The computed standard deviations of each HMI-Crack Width model for each of the four specimens, along with the average standard deviation of the respective HMI-Crack Width model are presented in Figure 6.13e. The HS-S-M model, or monotonic model, proved to have the lowest standard deviation consistently through the monotonically loaded smallscale program. Although based on the computed standard deviation of beam HS-S-M-4, the cyclic model is suggested to provide slightly better results. Upon investigation of the plotted results for beam HS-S-M-4, the cyclic model is found to underestimate the crack width, and thus the HS-S-M model would actually provide the more accurate representation of the internal behavior. The deviations computed for the HS-S-M-3, and HS-S-M-4 members show almost identical results, and yielded the lowest values of the four specimens in favor of the model developed from the monotonic specimens. The experimental results from the monotonically loaded small-scale members suggest that the equation developed based on the results of the monotonically loaded beams yield good results for crack width approximation. 


\subsubsection{Verification of Crack Width-HMI Relationship Based on Results Obtained from the Cyclically Loaded Small Scale Beams}

A similar analysis to the one in the previous section was carried out with the data obtained from the cyclically loaded members. The two HMI-Crack Width relationships, in Equation 6.2 and 6.3 , were verified with the experimentally measured crack widths obtained from the crack gauges. The results of this analysis are presented in Figure 6.14, along with a comparison of the two suggested equations to the directly measured values. The analysis was only carried out for the portions of the load procedure as the piezo-sensors were found to only be effective at capturing the maximum inflicted damage experienced by the section during the loading application stage, and showed little to no change in the HMI values during the unloading stage.

The results obtained from beam HS-S-C-1 are presented in Figure 6.14a. From the figure it is apparent that the results of the piezo-sensors are in good agreement with those of the crack gauges. Both systems presented results up to the failure load of the $260 \mathrm{kN}$ and seem to be in good agreement to one another. Both systems show almost identical results up to a maximum crack width of $0.3 \mathrm{~mm}$ and at the $150 \mathrm{kN}$ load step after which the piezo-system slightly underestimates the crack width consistently for both HMI-Crack Width models. Since the HS-S-C model was developed from the cyclically loaded members, it was expected to provide the best estimation of crack width for the specific experimental program, although this is not the case. As demonstrated by Figure 6.14a, the HS-S-C equation tends to underestimate the member's crack width by a much larger value than the HS-S-M model, and thus the HS-S-M equation, developed from the monotonically loaded beams, provides much better results. Although the values for the standard deviation of the data in Figure 6.14d suggests otherwise. Here it can be seen that the value of standard deviation of the HS-S-C model is almost half of that of the HS-S-M model, although the trend observed from the Load-Crack Width graph in Figure 6.14a would suggest the cyclic model is not as effective for the analysis.

The results obtained from beam HS-S-C-2 are presented in Figure 6.14b. Here the results of the two measurement systems, although follow the same trend, do not yield similar results, 
making this test the outlier of the series. The results obtained from the crack gauges are shown to be very consistent from one side of the beam to the other, although the piezoceramic readings are underestimating the crack width. Form the data series it can be concluded that the HS-S-M equation provides a more accurate representation of the internal crack width. The standard deviation of the data further confirms the conclusion supporting a stronger correlation for the HS-S-M equation.
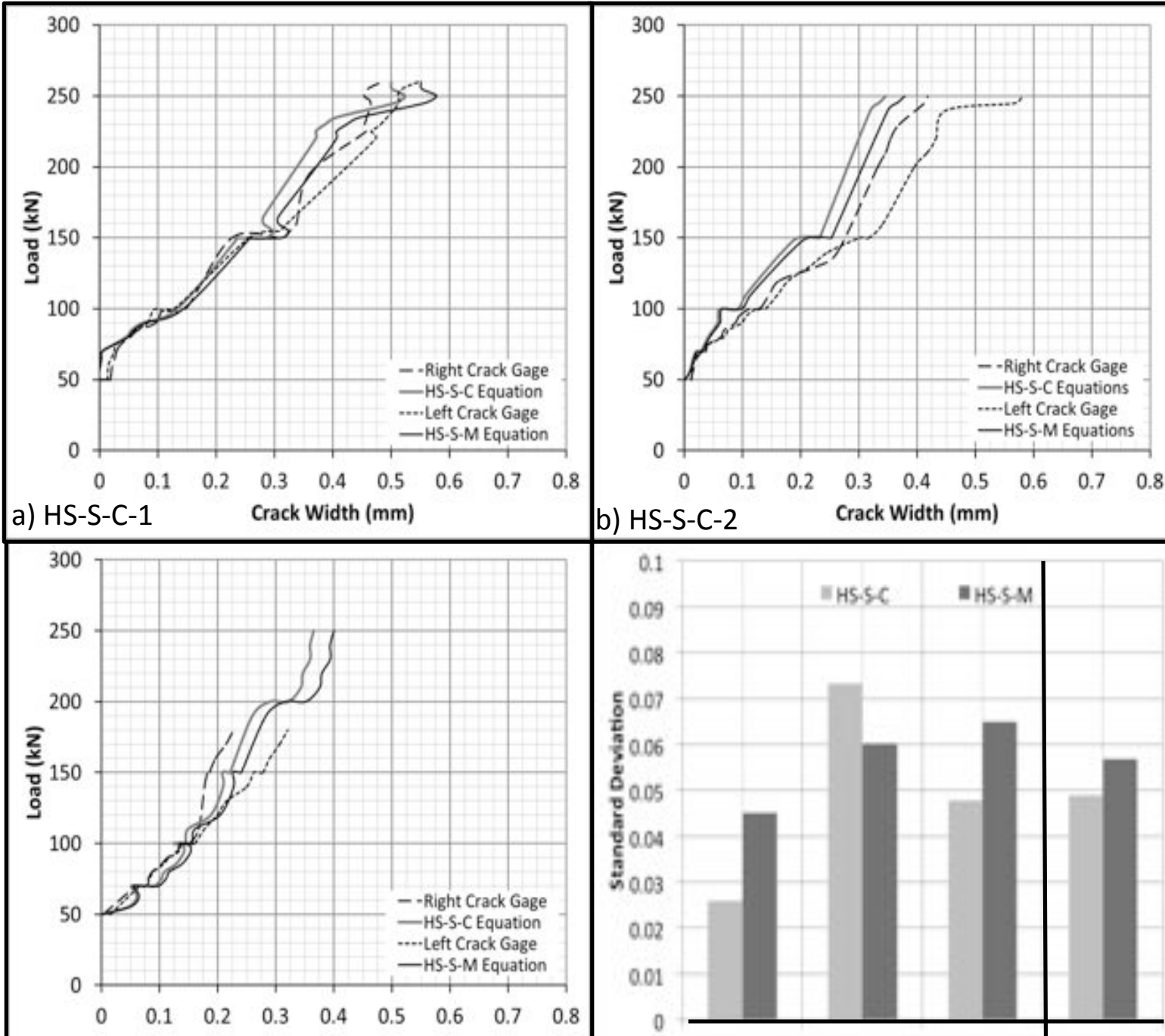

c) HS-S-C-3

Crack Width $(\mathrm{mm})$

d)

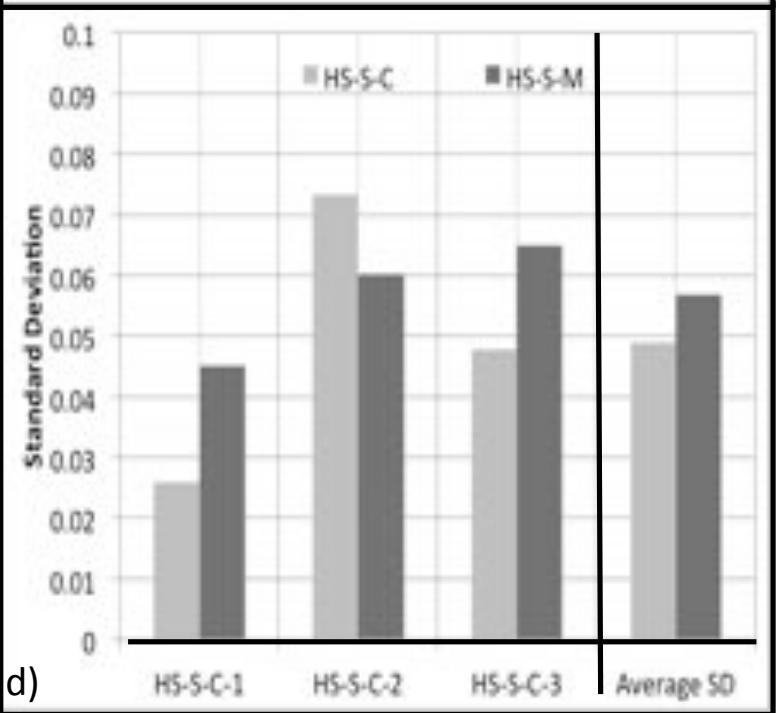

Figure 6.14: Comparison of HS-S-M and HS-S-C HMI-Crack Width Models, and Direct Crack Width Readings Using Crack Gauges, Using Experimental data from HS-S-C Beams; a) Beam HS-S-C-1; b) HS-S-C-2; c)HS-S-C-3; d) Computed Standard Deviation of Cyclic and Monotonic Models 
The crack gauge and piezo-sensor's results for beam HS-S-C-3 are shown in Figure 6.14c. The results obtained from the two crack width models are seen to correlate very well with the results obtained from the crack gauges. The piezo-sensors are expected yield results reflecting the average crack width value between the two crack gauges, and in Figure 6.14c the behavior is clearly visible. Although comparing the HS-S-M and HS-S-C equations shows that the cyclic model is more accurate of the expected internal crack width. This is also evident from the standard deviation of the two series. Where the standard deviation of the HS-S-C model is approximately two thirds of that calculated for the HS-S-M model. The analysis carried out, using the results of the cyclically loaded members, does in fact yield that the model for HMI-Crack Width developed from the cyclic experimental program shows a stronger correlation to the results obtained from the crack gauges. Although the difference between the two models is very small, and the visually observed correlation from the Load-Crack Width plots is favorable for the HS-S-M model as the model proves to be more accurate at higher values of crack width.

\subsubsection{Recommendation for a General Crack Width-HMI Equation}

The general HMI-Crack Width model is developed based on the results of all seven smallscale samples. The results obtained from the small-scale set of experiments provided two distinct relationships between the internal concrete strain, and the $\mathrm{HMI}$ output. The first relationship was deduced form the results obtained from the four monotonically loaded beams, and the second relationship was gathered form the three beams tested as part of the cyclic experimental program. The two HMI-Strain relationships were then applied to the $\mathrm{HMI}$ reading obtained throughout each test, and compared to the direct measurements of crack width taken from the crack gauges. Using the calculated standard deviations between the average crack width of a specimen and the results yielded each of the HMI-Crack Width models, along with the observed results of the Load-Crack Width graphs, one general model could be selected to be used with the piezoceramic system for crack width evaluation.

The analysis showed that both the monotonic and cyclic HMI-Crack Width models yielded acceptable results. Although, it was found that the cyclic model would slightly 
underestimate the effective crack width. The analysis carried out using the HMI data obtained from the monotonically loaded members confirmed that the monotonic HMICrack Width model is more effective for crack width estimation. The model provided good consistency with the direct crack width measurements of the crack gauges, as expected. Although, the data obtained from the cyclic beams did not suggest that the cyclic HMI-Crack Width model was preferred, but rather confirmed that the monotonic HMI-Crack Width equation was more accurate for measuring the crack width. Therefore, the equation obtained from the monotonic data is considered to be the more accurate, and will be adopted for the analysis of the large-scale samples. The relationship is summarized in Figure 6.15, along with the Equation 5.4 that should be used when converting the HMI values to crack with measurements.

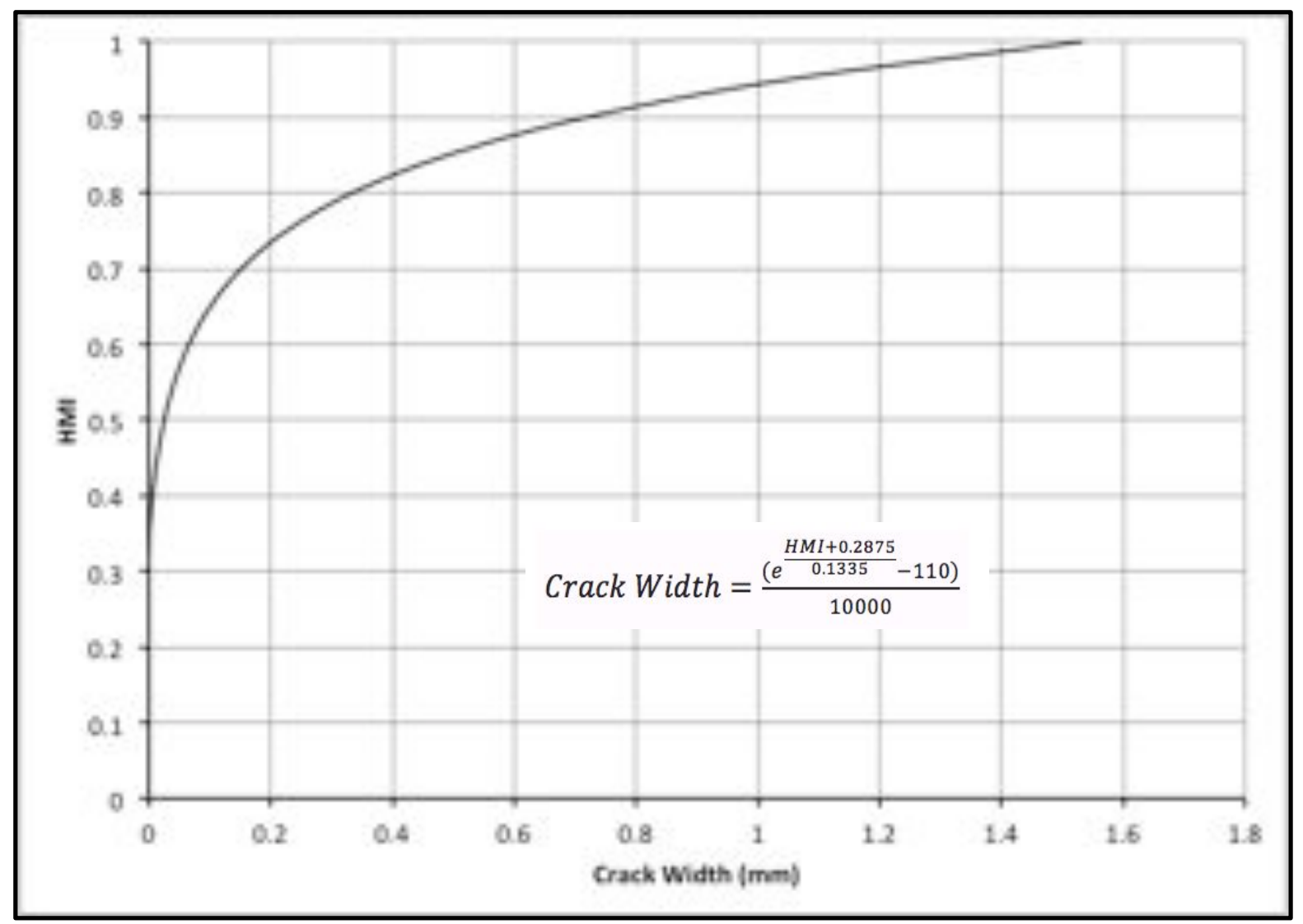

Figure 6.15: Universal HMI-Crack Width Relationship for Piezo-System 


\subsection{Full Scale Monotonic Load (HS-L-M)}

The HS-L-M experimental program consisted of two full-scale beams that were tested under monotonic loading routine, and carry the designation "HS-L-M-\#". Ten piezo-sensors were embedded into the concrete matrix of the full-scale beam, along with five multiplexed FBG fiber optic sensors. The members were loaded up to their respective failure loads in $25 \mathrm{kN}$ increments, and the results of the measuring equipment is presented herein. The two beams had a cross-section of $350 \mathrm{~mm}$ wide $\times 700 \mathrm{~mm}$ deep, total length of $4000 \mathrm{~mm}$, and identical flexural reinforcement detailing. The main difference between the two members was the shear reinforcement detailing. Beam HS-L-M-1 was designed to have inadequate shear reinforcement, predominantly exhibiting shear cracking. However, beam HS-L-M-2 was provided sufficient shear reinforcement as to exhibit flexural behavior.

The two full-scale beams were used to check the effectiveness of the proposed piezosensors, as part of a complete full-scale health monitoring system. The system was expected to predict the initiation of cracking, capture the appearance of cracking, and quantify the damage in terms of the developed HMI-Crack Width model from the smallscale experimental programs. The beams also provided the ability to demonstrate the location-based health monitoring capability of the proposed piezoceramic system. The sensors outputs obtained from beam HS-L-M-2 are compared to the Limit State design previsions provided by the Canadian Building Code, as to determine if the calculated limits can be differentiated solely based on the signal outputs of the piezo-sensors. For detailed calculations of the various Limit States please refer to Appendix B, the results of which are summarized in Table B-1. Appendix C presents the Calculations for average crack spacing, and average crack width for beam HS-L-M-2 for comparison purposes.

\subsubsection{HS-L-M-1}

Beam HS-L-M-1 was designed to contain less than the minimum shear reinforcement specified by the CSA-A24. The purpose of this study was to determine the piezo-system's effectiveness for shear crack detection, and quantification. The study would aid in the 
understanding of predictive health monitoring of shear failure using the proposed system. The appearance of shear cracking within a concrete member, and the initiation of a shear failure, are two the most abrupt and undesirable failure mechanisms of reinforced concrete members. As the mechanism of a shear failure provides little warning, the occurrence of such an event needs to be very thoroughly monitored, using reliable and accurate equipment. From previous investigations, the piezoceramic system was found to be effective for predicting the initiation of cracking in a concrete sample, and would provide an excellent means of monitoring the initiation of shear cracking. By quantifying how close a member is to experiencing shear cracking, maintenance personal can effectively rehabilitate a damaged section as to avoid a possible future shear failure of a concrete member.

\subsubsection{Member Behavior}

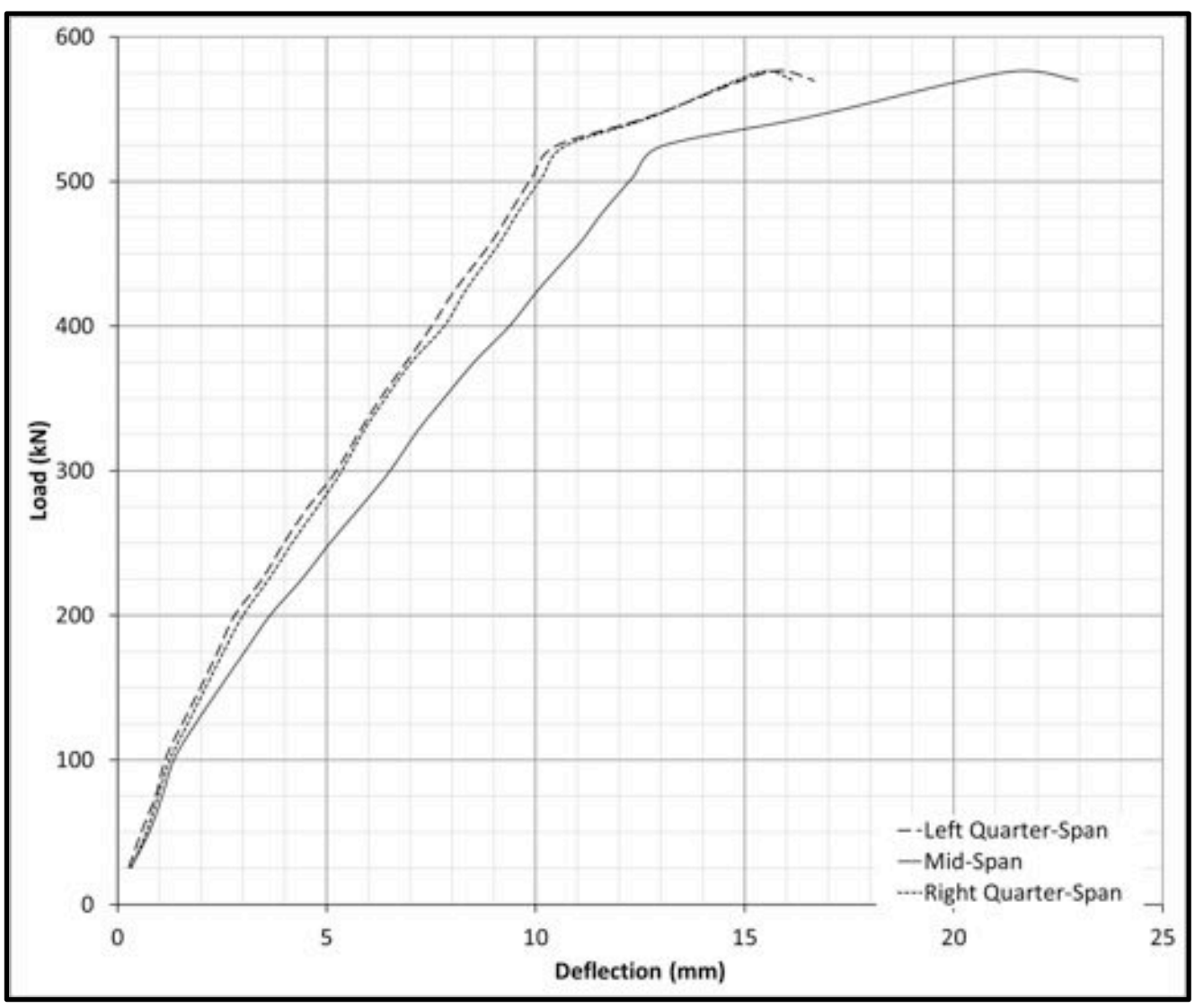

Figure 6.16: Load-Defection Curve for Beam HS-L-M-1 
The Load-Deflection curves for beam HS-L-M-1 is presented in in Figure 6.16. A total of three LPDTs were installed, recording the deflection at the mid-span, and the two quarterspans of the beam. The three LPDTs would verify that the desired behavior was achieved through the experimental work. From the Load-Deflection graph, beam HS-L-M-1 is witnessed to have a failure load of $525 \mathrm{kN}$, with a mid-span deflection of $13 \mathrm{~mm}$, and quarter-span deflections of $10.47 \mathrm{~mm}$ and $10.69 \mathrm{~mm}$. The member initiated cracking at the load step of $150 \mathrm{kN}$ agreeing with the calculated cracking load of $167.30 \mathrm{kN}$. From the load deflection graph the quarter-span and mid-span deflections are similar up to a load of 110 $\mathrm{kN}$ after which the quarter-spans deflect at a much slower rate than the mid-span of the beam. Overall the beam acted as expected throughout the experimental period, initiating shear cracking at a load of $250 \mathrm{kN}$, when the calculated shear capacity of the concrete section was $163 \mathrm{kN}$.

\subsubsection{Piezo-Sensors Location and Results}

The ten piezo-sensors in member HS-L-M-1 were distributed as to predominantly monitor the appearance of the shear cracking. The final positions of the ten piezo-sensors are detailed in Figure 6.17. A total of eight signal paths were chosen for the investigation of the beam. Sensor pairs 1-2 and 9-10 monitored the area beside support locations, and were not expected to detect any cracking as the pairs were intended to provide a control for the experiment. The control would provide a good means of investigating the validity of the 0.5 cracking threshold established from the small-scale members. Sensor pairs 2-3 and 8-9 monitored the appearance of the shear crack at a distance of $d_{v}$ from the support, along with some basic information of the appearance of flexural-shear cracks. The main focus of the study conducted with member HS-L-M-1 were sensor pairs 3-4 and 7-8, as the two sensor pairs were positioned to monitor the appearance of shear cracking exclusively, and would establish a good comparison with the readings obtained from the fiber optic cable. The final signal paths chosen were between sensor pairs 4-5 and 6-7 located in the compression block of the beam. Compressive strain was not studied from the small-scale experimental program and thus little is known about the behavior of the piezo-sensors 
when exposed to this behavior. The intended purpose of the sensors was to detect the appearance of concrete crushing caused by high compressive strains developed through the compressive block, causing a certain member failure. Since a limit for the $\mathrm{HMI}$ is not known for a section undergoing compressive strain, a separate investigation needs to be carried out.

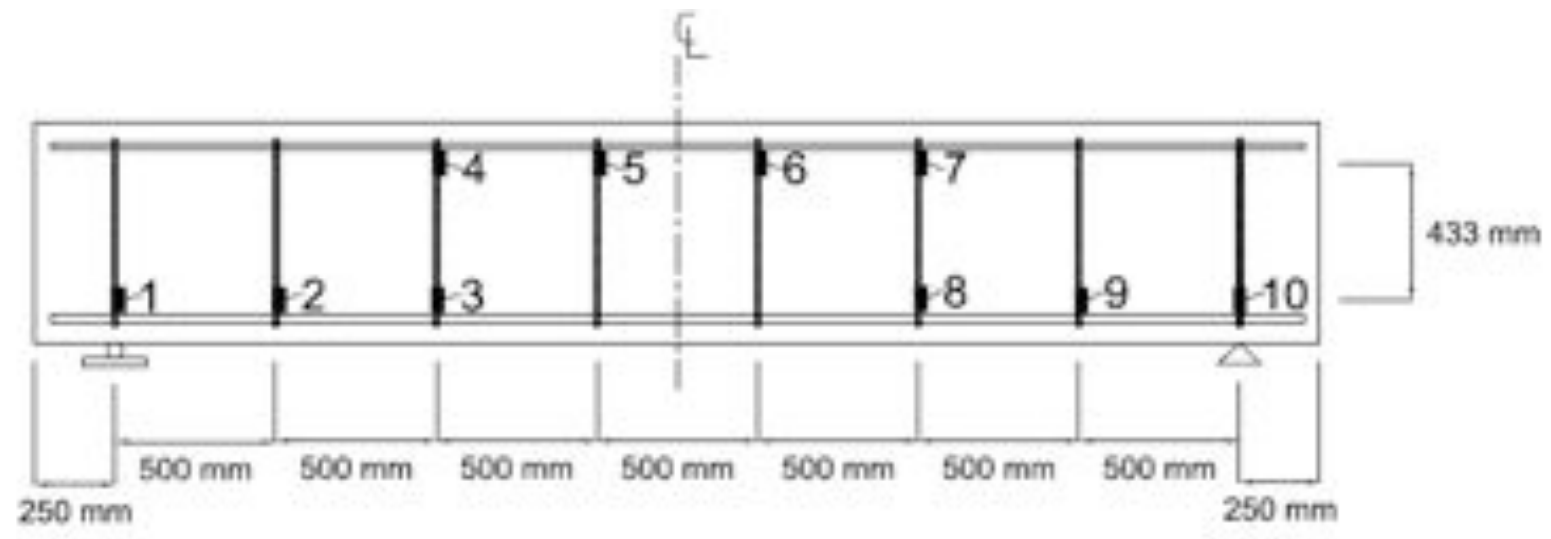

Figure 6.17: Piezo-Sensor Spatial Position

The piezo-sensors demonstrate a very linear response during the un-cracked duration of the member's lifetime. Based on the response of sensor pair 3-4 and 7-8, the response of the system is almost linear from the HMI value of 0 up to the cracking threshold of 0.5 . This linearity of the output signal can prove to be very useful for non-destructive evaluation, especially in the case of shear crack evaluation. As the mechanism leading to a shear failure of a concrete beam is very sudden, and providing little warning before its inevitable occurrence, a means of quantifying the risk that a beam may experience such a failure is vital. The linearity of the output signal, along with the threshold established, provide the basis for a crack prediction system. The piezo-system trigger a warning to show how close a member is to cracking solely on the value of HMI output; as the HMI value approaches the 0.5 benchmark, the probability of a crack to appear in the specific region increases. Thus the strategic placement of these sensors will declare how close a member is to cracking, and if the location monitored is the beam's critical location, the system will gauge how close a building's system is to failure. All the sensor pairs in beam HS-L-M-1 consistently exhibit the linear behavior of the output signal during the un-cracked stages of the beam. 


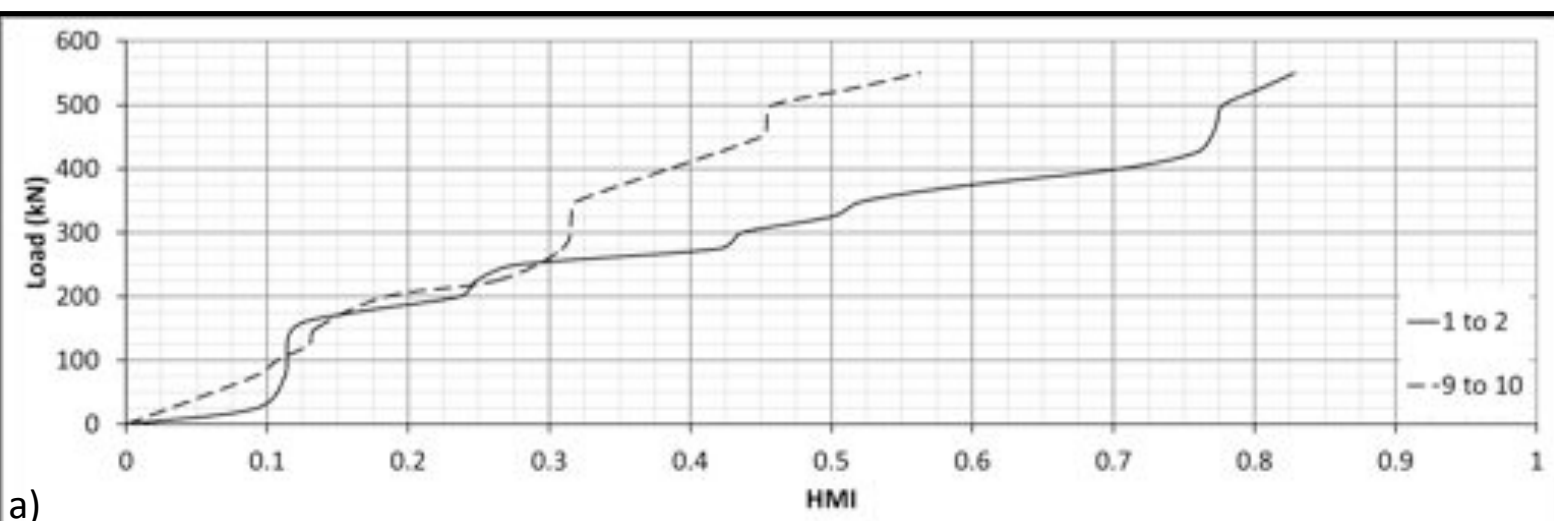

a)

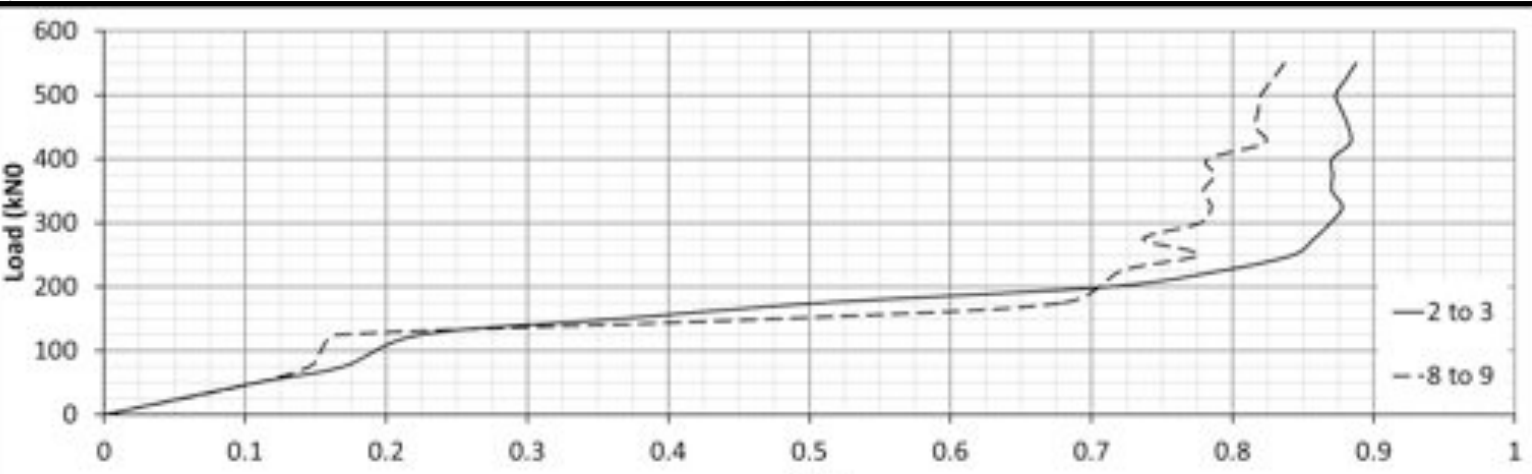

b)

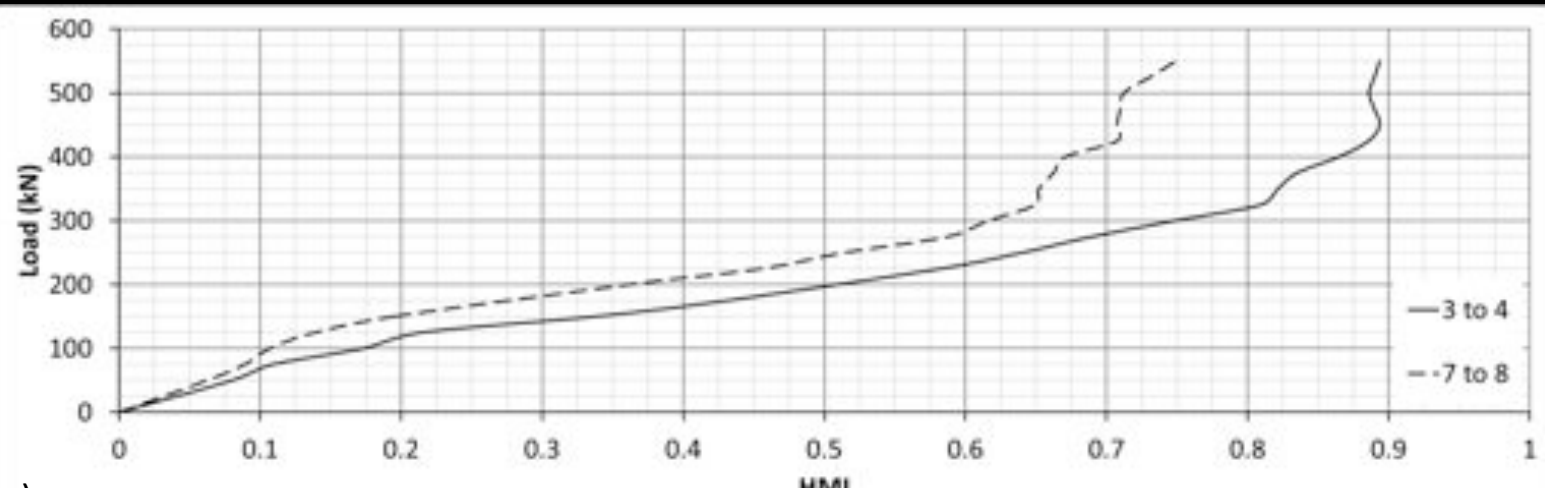

c)

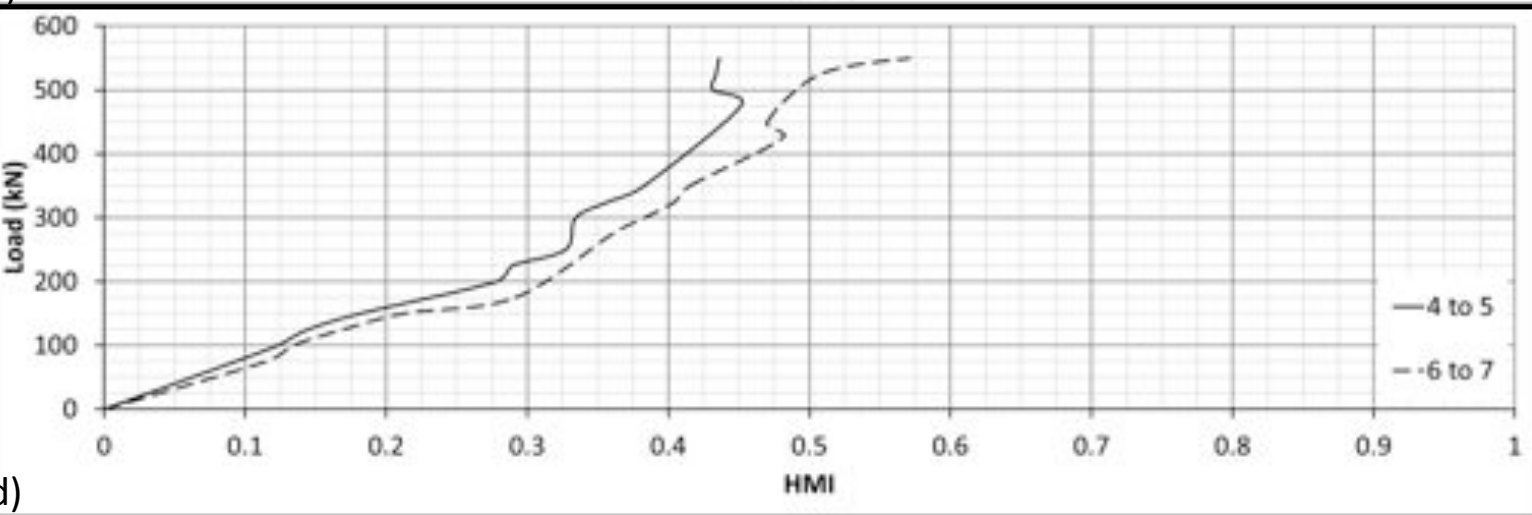

Figure 6.18: Load-HMI Graphs for HS-L-M-1; (a) Support Location; (b) Quarter-Span; (c) Shear Crack; (d) Compression Block 
Sensor pairs 3-4 and 7-8 were the most important sensors pairs in the non-destructive evaluation of beam HS-L-M-1, as they monitored the initiation of the shear crack. The Load$\mathrm{HMI}$ graphs for these sensor pairs are presented in Figure 6.18c. The initiation of the shear cracks was visually observed at the load step of $300 \mathrm{kN}$ for the left half of beam HS-L-M-1, and at $250 \mathrm{kN}$ for the right half. From the Load-HMI graph, the surface opening cracks yielded $\mathrm{HMI}$ values of 0.75 , and 0.51 for the left and right halves of the beam respectively. The HMI values, although quite different, are both indicative of a cracked section. Using the 0.5 threshold established from the small-scale experimental program, as the primary source to evaluate the member's condition, the left half of the beam would have been considered cracked at the $225 \mathrm{kN}$ load step, and the right half would have been considered crack at the $250 \mathrm{kN}$ load step, both providing a warning of a possible future failure at much earlier stages than conventional means.

The Load-HMI graphs obtained from these sensor pairs are shown in Figure 6.18b The section monitored by sensor pair 2-3 was found to have two cracks initiate through the signal paths at load steps of $225 \mathrm{kN}$, and $300 \mathrm{kN}$ yielding $\mathrm{HMI}$ values of 0.79 , and 0.87 , respectively. The opposing side of the beam monitored by sensor pair 8-9 was observed to crack at $175 \mathrm{kN}, 225 \mathrm{kN}$, and $300 \mathrm{kN}$, yielding HMls of 0.68,0.72, and 0.78, respectively. Similar to the results described from sensor pair 3-4, the HMI values for sensor pair 2-3 crosses the 0.5 threshold at a load of $175 \mathrm{kN}$, and would suggest that the beam's cracking load was in fact $175 \mathrm{kN}$, not the visually observed $225 \mathrm{kN}$ at this section. Sensor pair 8-9 exceeded the 0.5 thresholds at a load of $150 \mathrm{kN}$. Both sensor pairs, monitoring the initiation of cracking in the effective shear zone providing warning for the initiation of the shear crack 25-50 kN prior to the crack being visible on the exterior of the beam. The remaining sensor pairs demonstrated that the piezo-system is effective for the detection of shear cracking in a reinforced concrete member.

Sensor pairs 1-2, and 9-10 monitored the sections of the beam directly adjacent to the support. Since beam HS-L-M-1 was designed to fail in shear, due to its lack of adequate shear reinforcement, the region above the supports was expected to crack once the shear capacity of the concrete section was exceeded. Although no cracking was observed visually 
between sensor 9 and 10, the Load-HMI graph in Figure 6.18a demonstrates that the beam cracked at a load of $525 \mathrm{kN}$. This result agrees with the calculated failure load of $530 \mathrm{kN}$. Sensor pair 1-2 demonstrated similar results. As the beams ultimate capacity was reached, two cracks appeared near the left support of the beam. The first crack was observed to cross sensor 2 at the $400 \mathrm{kN}$ load step and yield an $\mathrm{HMI}$ value of 0.70 , and the secondary crack was observed to intersect the signal path of the sensor pair at $550 \mathrm{kN}$ yielding an $\mathrm{HMI}$ value of 0.83 . Based on the results of the piezo-sensors, the HMI value for sensor pair $1-2$ exceeded the 0.5 cracking limit at a load of $325 \mathrm{kN}$, suggesting this to be the cracking load for this signal path.

The results obtained from the sensor pairs monitoring the compression zone, 4-5, and 6-7 are provided in Figure 6.18d. Here sensor pair 6-7 exceeds an HMI of 0.5 at $525 \mathrm{kN}$. Since sensor pairs, 9-10 and 6-7 exceeded the 0.5 thresholds, the results suggest that the member cracked internally beside the right support resulting in a shear failure. The results between sensors pair 1-2 yielded different results. A surface opening crack was observed at a load of $400 \mathrm{kN}$ yielding a $\mathrm{HMI}$ value of 0.71 . The results obtained by the HMI values both suggest that the shear crack did not cause concrete crushing between sensors 4-5, and the crack between sensor pair 1-2 was captured at the $325 \mathrm{kN}$ load step. The results provide that the system is effective for predicting, and capturing the initiation of shear cracking, and providing a means of predicting a beam's shear failure

\subsubsection{FBG Fiber Optic Sensors}

A total of 5 fiber optic sensors were installed in beam HS-L-M-1. The sensors were intended to monitor the shear crack, quarter-span, and mid-span location of the beam. The results of these sensors were intended to provide a comparison between the crack widths measurements provided by the piezo-system using the proposed HMI-Crack Width relationship, and the direct measurements of crack width provided by the fiber optic sensors. The five fiber optic sensors were multiplexed into one continuous cable. The continuous cable was easily installed into the concrete matrix as to monitor the desired 
locations, although due to the fragility of the cable, only one of the five sensors worked following the casting process. Since the cable was multiplexed, when the cable is ripped in any one specific location, the sensors following the damaged location will not function. The sensor installed in beam HS-L-M-1 was torn after the first fiber optic sensor, and thus the remaining sensors did not work. Figure 6.19a shows the location of the working FBG sensor. The FBG sensor monitored the concrete strain experienced perpendicular to the shear crack. The Load-Strain graph is presented in Figure 6.19b.

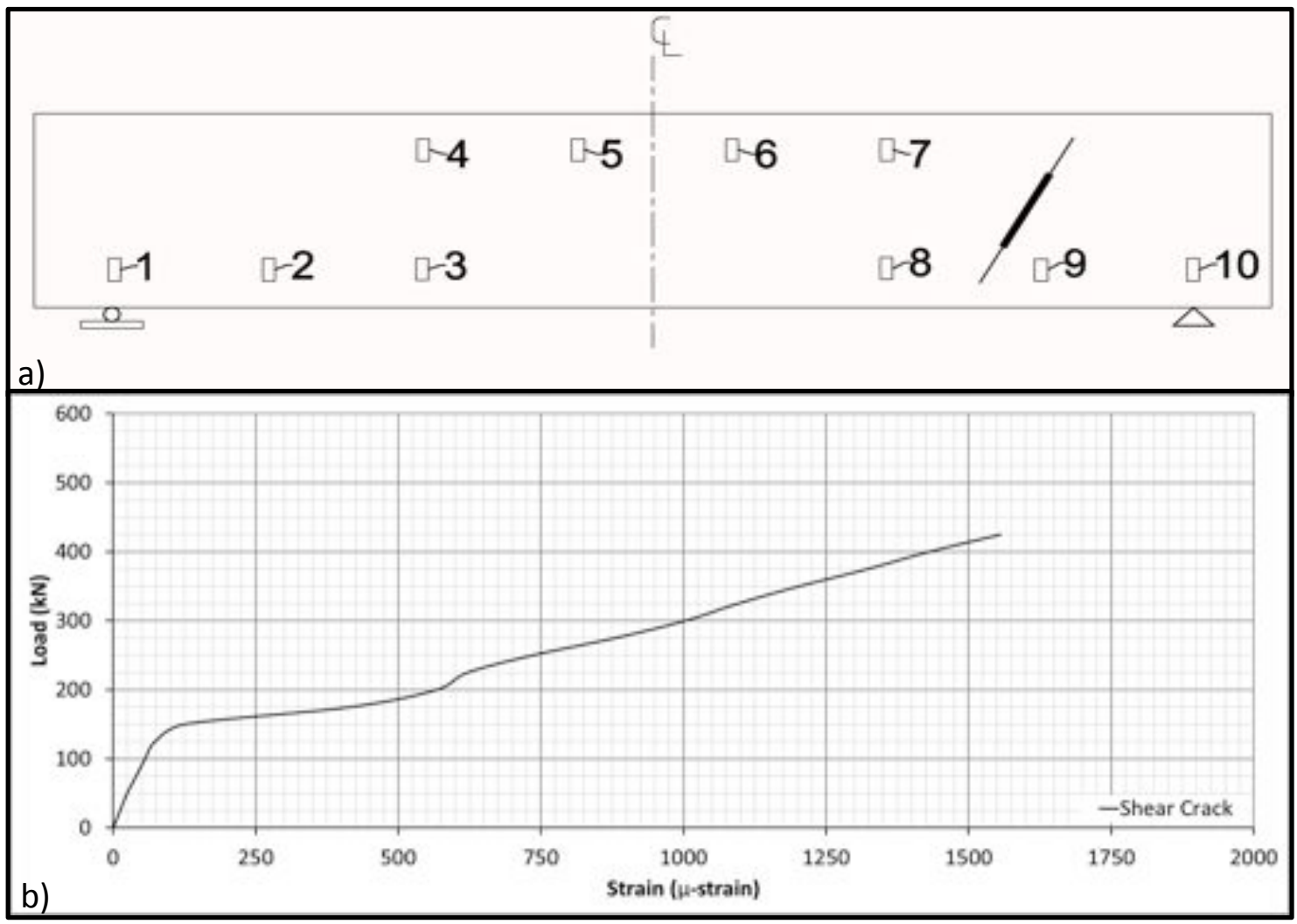

Figure 6.19: FBG Fiber Optic Readings from Beam HS-L-M-1; (a) Location of Working Sensors; (b) Readings Obtained from FBG Sensor

The results obtained from the FBG fiber optic sensor show that the concrete strain across the location of the shear crack exceeded the $110 \mu$-strain limit at the $150 \mathrm{kN}$ load step. The sensor proved to be very effective for detecting the initiation of cracking, as the shear crack propagation was only visible after the $250 \mathrm{kN}$ load step. The result obtained from the fiber optic sensor agrees with the results obtained from sensor pair 8-9 monitoring the effective 
shear depth location from the right support. The sensor pair yielded that a crack initiated at this location at the $150 \mathrm{kN}$ load step. The fiber optic sensor was effective to the $425 \mathrm{kN}$ load step providing readings up to $1560 \mu$-strain. The results of the fiber optic sensor are expected to be comparable with the ones obtained from sensor pair 7-8 monitoring the shear crack.

\subsubsection{Crack Width Measurements Using Piezo-System and Member Cracking Behavior}

The small-scale experimental program helped develop a universal HMI-Crack Width equation that can be used in the investigation of beam HS-L-M-1 to quantify the appearance of cracking in terms of an estimated crack width. The following investigation is carried out in order to check the validity of the model, and to demonstrate the abilities of the piezo-system. The focus of the HS-L-M-1 experimental program was to predict, and detect, the shear crack of a reinforced concrete beam. The HMI results obtained from the piezo-sensors demonstrated the system's ability to predict and detect the appearance of a shear crack. The system is required to quantify the damage detected in terms that could be easily interpreted such as a measure of crack width. The HMI results obtained from the six sensor pairs monitoring the tensile strains were converted to the equivalent crack widths using Equation 6.4. As the compressive strains were not yet investigated, and a model was not developed to interpret the results, sensor pairs 4-5 and 6-7 are not part of this investigation. The results of the FBG fiber optic sensors are converted to values of crack width, using its specified $300 \mathrm{~mm}$ gauge length.

The crack width measurements provided of the fiber optic cable, along with the results obtained from the equivalent piezo-sensor pairs are presented in Figure 6.20a. Figure 6.20b, and Figure 6.20c, show the cracking behavior of beam HS-L-M-1 around the location of signal paths 3-4 and 7-8. The crack width measurements provided by the fiber optic sensor found in beam HS-L-M-1 is expected to provide results agreeing with those obtained from the 7-8 signal paths. From the graph obtained in Figure 6.20a along with the cracking behavior in Figure $6.20 \mathrm{c}$, the results of the piezo-sensor agree with the ones obtained from 
the FBG cable. The fiber optic cable captured the initiation of the shear crack at the $150 \mathrm{kN}$ load step, and the piezo-sensors yield the crack's initiation at $175 \mathrm{kN}$.

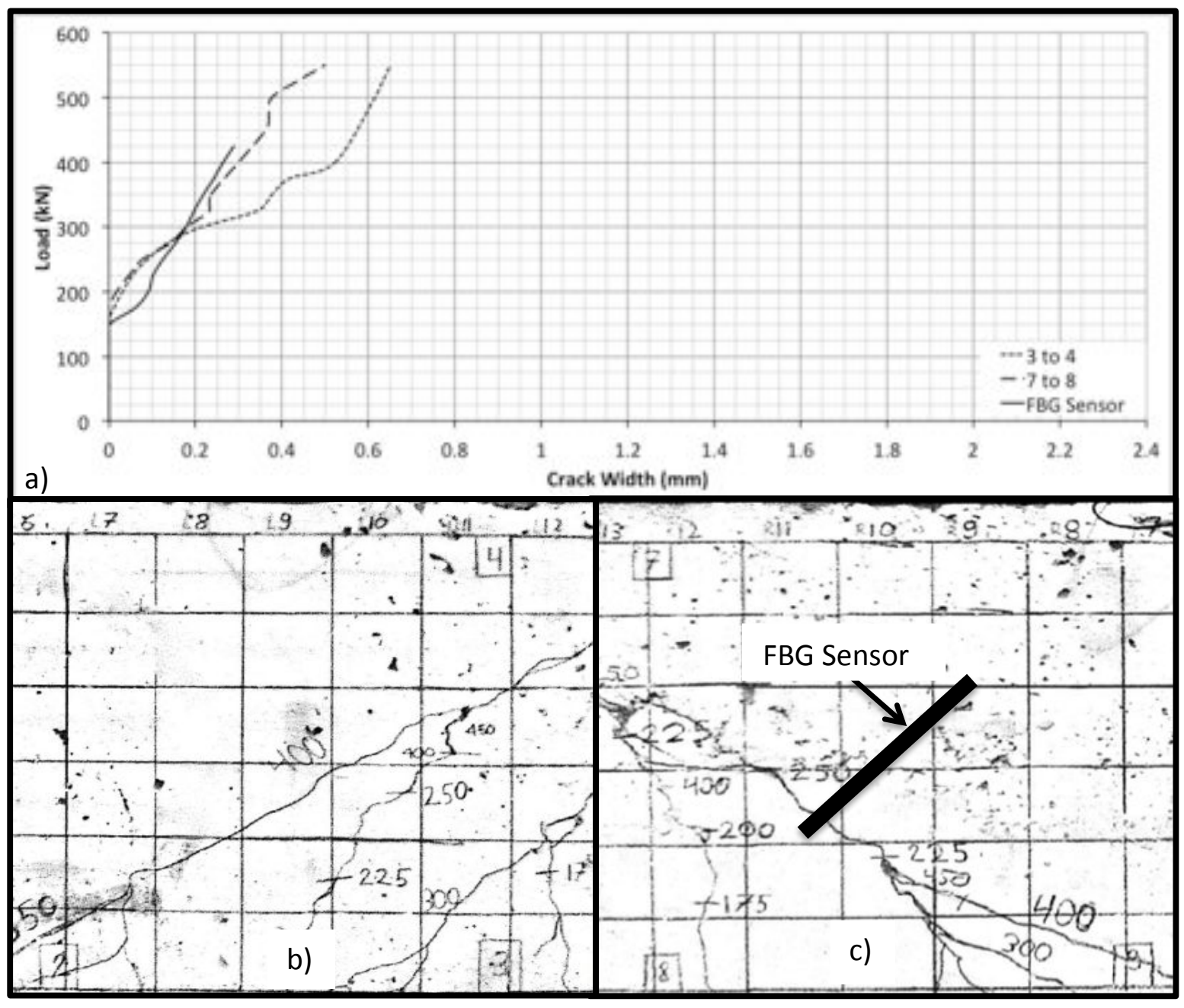

Figure 6.20: Shear Crack Behavior for Beam HS-L-M-1; (a) Crack Width Measurements; (b) Cracking Behavior Left Side; (c) Cracking Behavior Right Side

The difference in load is due to the location at which each system took reading. This difference in location between the reading obtained along the shear crack will cause the piezo-system to show results that are slightly lagged from those of the fiber optic cable, and this is seen to be very true up to a load step of $300 \mathrm{kN}$. As the beam undergoes the $300 \mathrm{kN}$ load step a secondary crack is found to intercept the signal path of the piezo sensors. The developed crack width model is based on the strain experienced between a pair of piezosensors, and thus, the readings obtained are representative of the collective strain over the 
monitored area. Therefore, the crack width measurements yielded by the sensor pair 7-8 are of the combined crack widths of the two cracks intercepting the signal path, and the results of the piezo-sensors are in very good agreement with those obtained by the fiber optic sensors.

The sensor pair monitoring the shear cracks on the left half of beam HS-L-M-1, also showed good agreement with the results obtained from the right half. The 3-4 sensor pair showed the initiation of a shear crack at a load of $150 \mathrm{kN}$, which is found to be in strong agreement with the results of the fiber optic cable from the symmetric side. Although just as discovered on the right half of the beam, the shear crack is not visible at the surface until a much higher load step. The signal path, just as on the symmetric side, was intercepted by a flexural-shear crack at the $300 \mathrm{kN}$ load step, and is reflected in the Load-Crack Width graph of the sensor pair, by the sharp increase in total crack width. Readings were taken with this sensor pair up to a load of $550 \mathrm{kN}$ resulting in a combined crack width of $0.65 \mathrm{~mm}$ at the failure load, compared to the total crack width of the 7-8 sensor pair of $0.45 \mathrm{~mm}$. demonstrating a much higher degree of damage incurred on the left half of the beam. The shear sensor pairs proved to be effective at capturing the propagation of the shear crack as well as quantifying the crack width incurred. Although the investigation carried out using sensor pairs 3-4 and 7-8 only provide a portion of the complete analysis of beam HS-L-M-1.

Sensor pairs 2-3 and 8-9 monitored the location beyond the effective shear depth of the beam, at the location the shear crack is expected to initiate. The two sensor pairs were exposed to a total of three cracks; one shear crack, and two flexural-shear cracks, are shown in Figure 6.21b and Figure 6.21c; corresponding to the cracking behavior on the left and right halves of beam HS-L-M-1 respectively. Signal path 2-3 was visually observed to be intercepted by cracks at 225 kN, 300 kN and 400 kN load steps. From the Load-Crack Width graph presented in Figure 6.21a, the sensors exhibit the initiation of cracking in this region at $150 \mathrm{kN}$, and show results for the combined crack width of the section up a value of 0.45 $\mathrm{mm}$ from where sensor 3 began to malfunction. The malfunction is caused by the appearance of an internal cracking across the piezo-sensor, causing it to lose bond with the concrete matrix. 


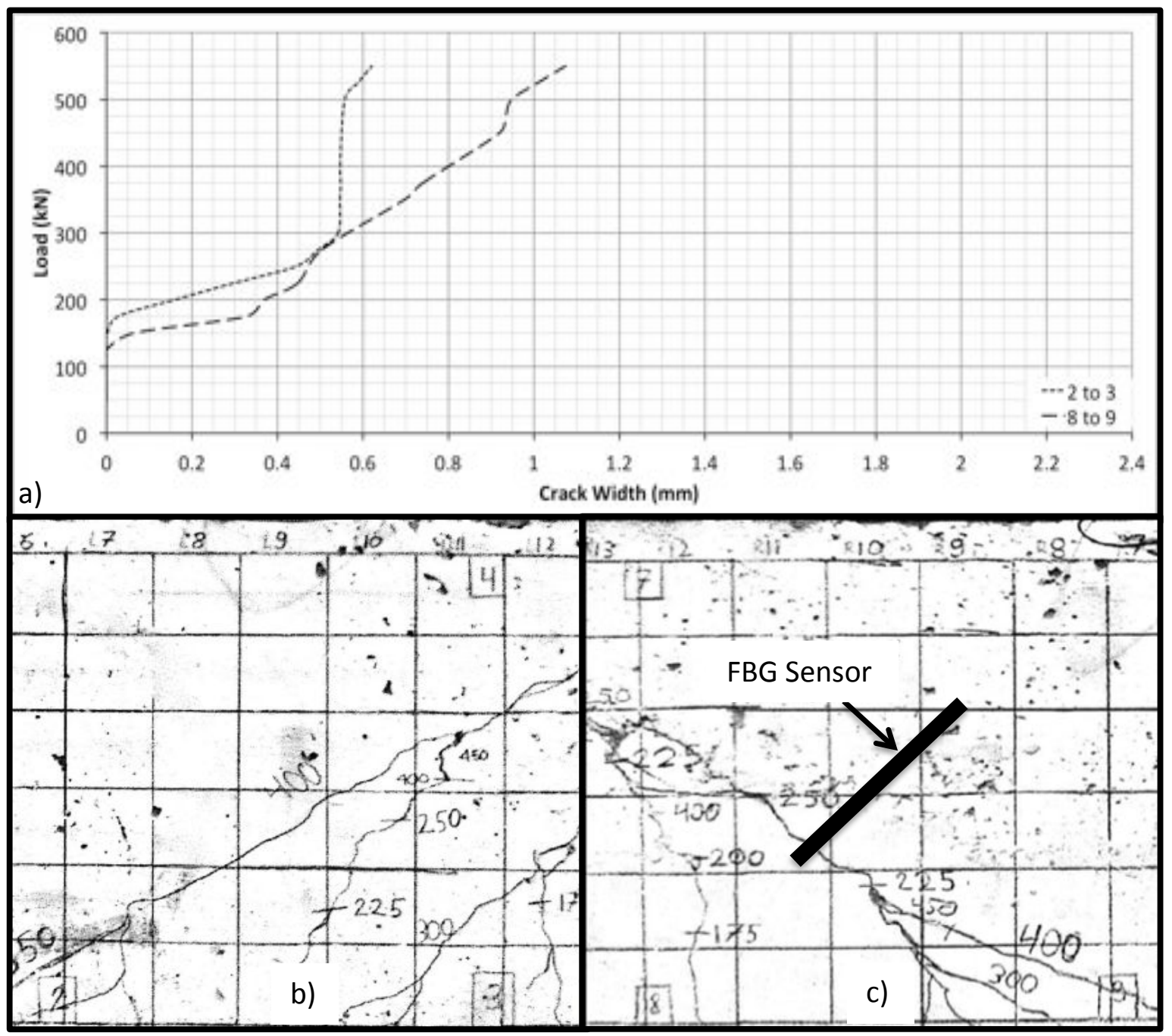

Figure 6.21: Effective Shear Depth Location Behavior for Beam HS-L-M-1; (a) Crack Width Measurements; (b) Cracking Behavior Left Side; (c) Cracking Behavior Right Side

Sensor pair 8-9 showed similar results, although the sensor pair did not malfunction through the experiment. Cracks were observed to intercept the signal path at the 175, 225, and $300 \mathrm{kN}$ load steps, and observed in Figure 6.21c. The initiation of cracking in this region was captured by the piezo-sensors at a load of $125 \mathrm{kN}$. In Figure 6.21a it can be seen that the calculated crack width captured by sensor pair 8-9 provides a consistent trend up to a combined crack width value of $1.14 \mathrm{~mm}$, and load of $550 \mathrm{kN}$. 


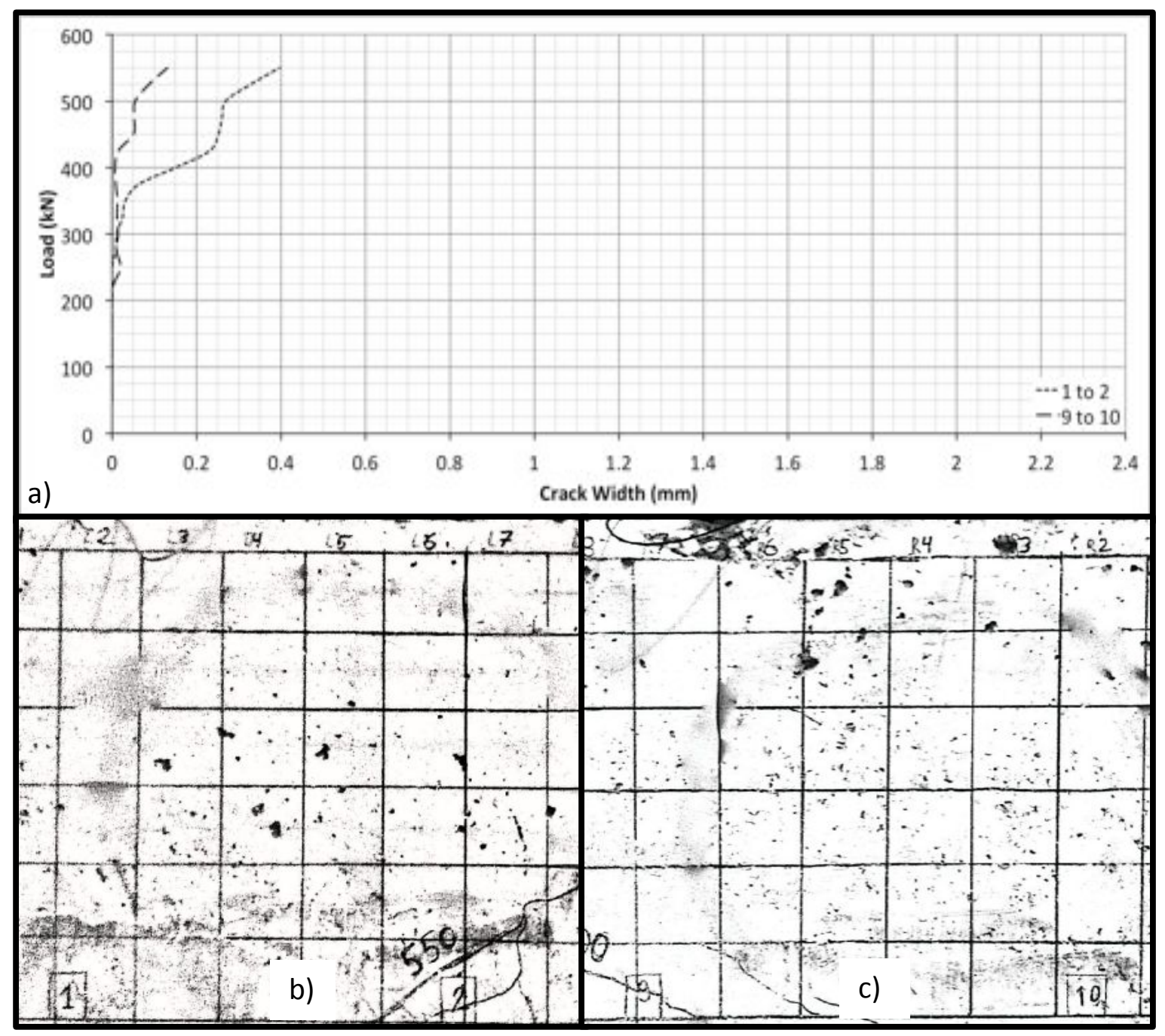

Figure 6.22: Support Location Behavior for Beam HS-L-M-1; (a) Crack Width Measurements; (b) Cracking Behavior Left Side; (c) Cracking Behavior Right Side

Although not much cracking was expected to occur in the $500 \mathrm{~mm}$ region adjacent to the beam's support, sensors were installed to monitor this region and provide a control measurement. The sensors were installed with the primary purpose of verifying the cracking limit for the HMI value established in the small-scale experimental program. Sensor pair 1-2 monitored the location near the left support of the beam, and sensor pair 9-10 monitored the location adjacent to the right support. The results of the two sensor pairs are presented in Figure 6.22a, along with the cracking behavior of the beam near the right and left support in Figure 6.22b, and Figure 6.22c respectively. The region, although not expected to exhibit cracking, did in fact crack at the late stages of loading. The location between sensor pair 1-2, 
demonstrated the highest degree of damage, capturing the shear crack that initiated at the $500 \mathrm{kN}$ load step and was visually observed at the $550 \mathrm{kN}$ load step. Although the crack was visually observed at the $550 \mathrm{kN}$ load step, it was thought to initiate much earlier during the loading program. A secondary crack initiated across the location of sensor number 2 at the $400 \mathrm{kN}$ load step. The combined crack width of the two cracks was measured using the piezo-sensors to be $0.4 \mathrm{~mm}$ at the $550 \mathrm{kN}$ load step.

The sensors monitoring the region beside the right support detected the appearance of cracking at the $525 \mathrm{kN}$ load step. The piezo-sensors captured the extension of the shear crack as the member was failing. The crack intercepted sensor number 9, and was detected by sensor pair 9-10. The two sensor pairs near the supports helped establish the reliability of the system. As the beam was loaded to its failure load, little to no change was witnessed in the signal output of this region. The results confirmed the initial assumption; little to no cracking activity was expected in this region. Although as the load of the member started to exceed the shear capacity of the beam, cracking was detected near the support location by the piezo-system, and confirmed by visual observation on the surface of the member.

\subsubsection{Results Obtained Using the MIRA 3D Tomographer}

The MIRA 3D tomographer was used to map the location of cracking within beam HS-L-M-1. Readings were taken using the equipment along the length of the beam as well as along the height. As the member is expected to behave relatively similar on both halves, only half the beam was scanned, and the results are presented here in. Along with mapping the location of cracking in the member, the MIRA was also used to determine the location of steel reinforcement. Reading were taken prior to the member being loaded, and after the loading program was complete, to provide a differentiation between the healthy and damaged state of the member.

Figure 6.23 illustrated the location of steel reinforcement found in beam HS-L-M-1 (Figure 6.23a) and the software output obtained from the MIRA software upon scanning the healthy state surface of the beam in the vertical direction (Figure 6.23b). This reading 
provides a means of establishing a reference that the damaged state of the beam can be compared to later, as to map the induced damage after loading. The only information that the MIRA output should provide is the location of the steel reinforcement in beam HS-L-M1. The tomographer was not able to provide enough detailed information to establish the location of the reinforcement based on the result obtained in the vertical scan direction. Although minor reflections of the steel reinforcement's location are present in the result, the information is not representative of a clear definition of the stirrups' locations. Thus the result can be used primarily as a reference for the mapping of the cracking pattern.

a)

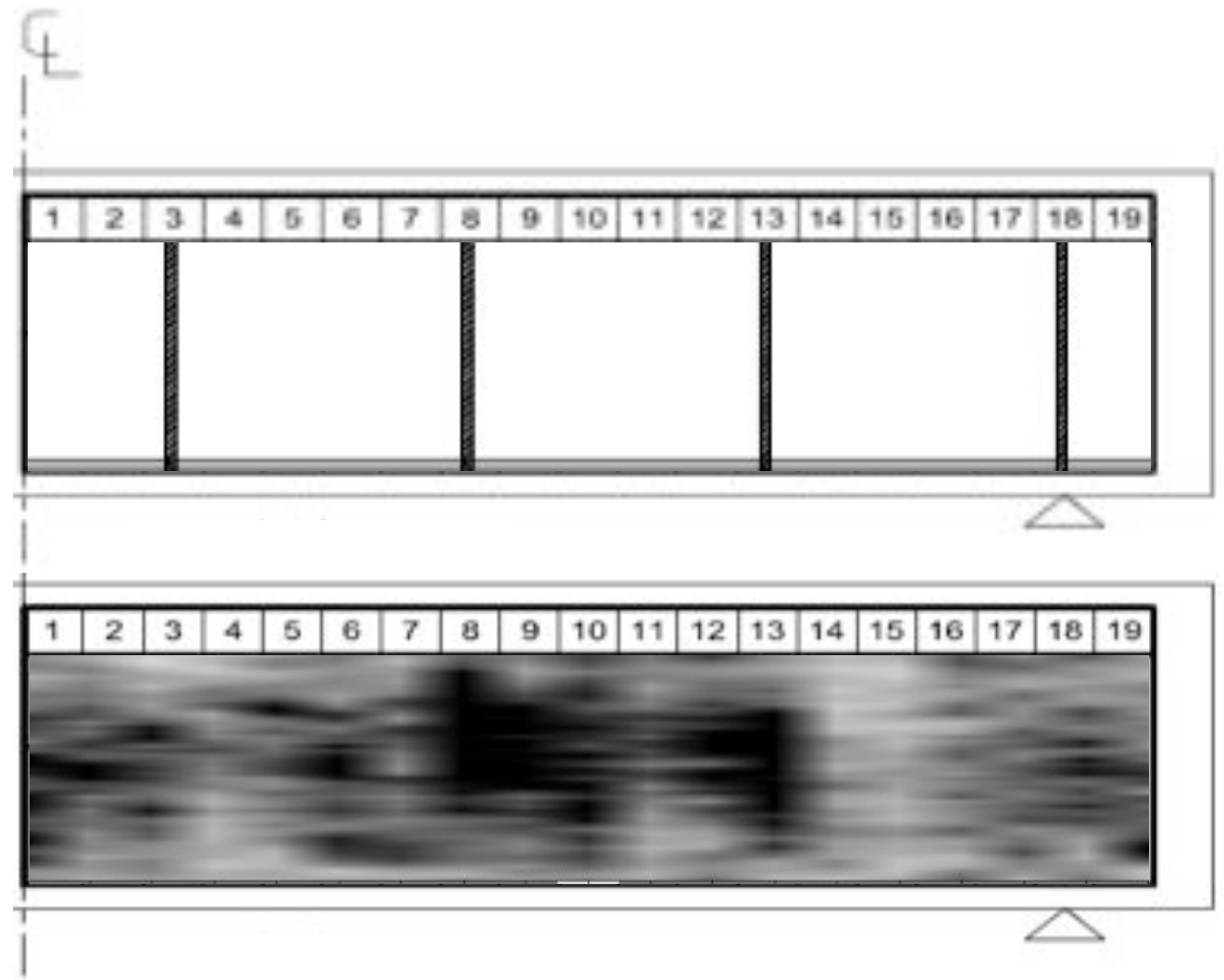

Figure 6.23: Vertical Scan Direction Beam HS-L-M-1 Healthy State; (a) Theoretical Location of Steel Reinforcement; (b) MIRA Software Output

Figure 6.24 illustrates the damaged state results obtained from the observed cracking pattern (Figure 6.24a) of the beam and the MIRA tomographer (Figure 6.24b). The MIRA tomographer is only ideally expected to detect cracking that is perpendicular to the 
direction of the scan. Since the software output presented in Figure $6.24 \mathrm{~b}$ is for the vertical direction of scanning, horizontal cracks should be detected (i.e. shear cracking). Based on the working principal of the equipment, a high amount of signal reflection should be reflected on either side of the cracking region between sections 5 and 12 of the beam. Based on the cracking pattern of the beam and the results of the MIRA tomographer, a slight variation is noticed in the software output by the equipment, depicted with the three white squares in Figure 6.24b. Slight increases in reflections in this region are visible as compared to the healthy state tomograph, suggesting the appearance of cracking, although the information is not clear enough to establish the extent of the damage.

a)

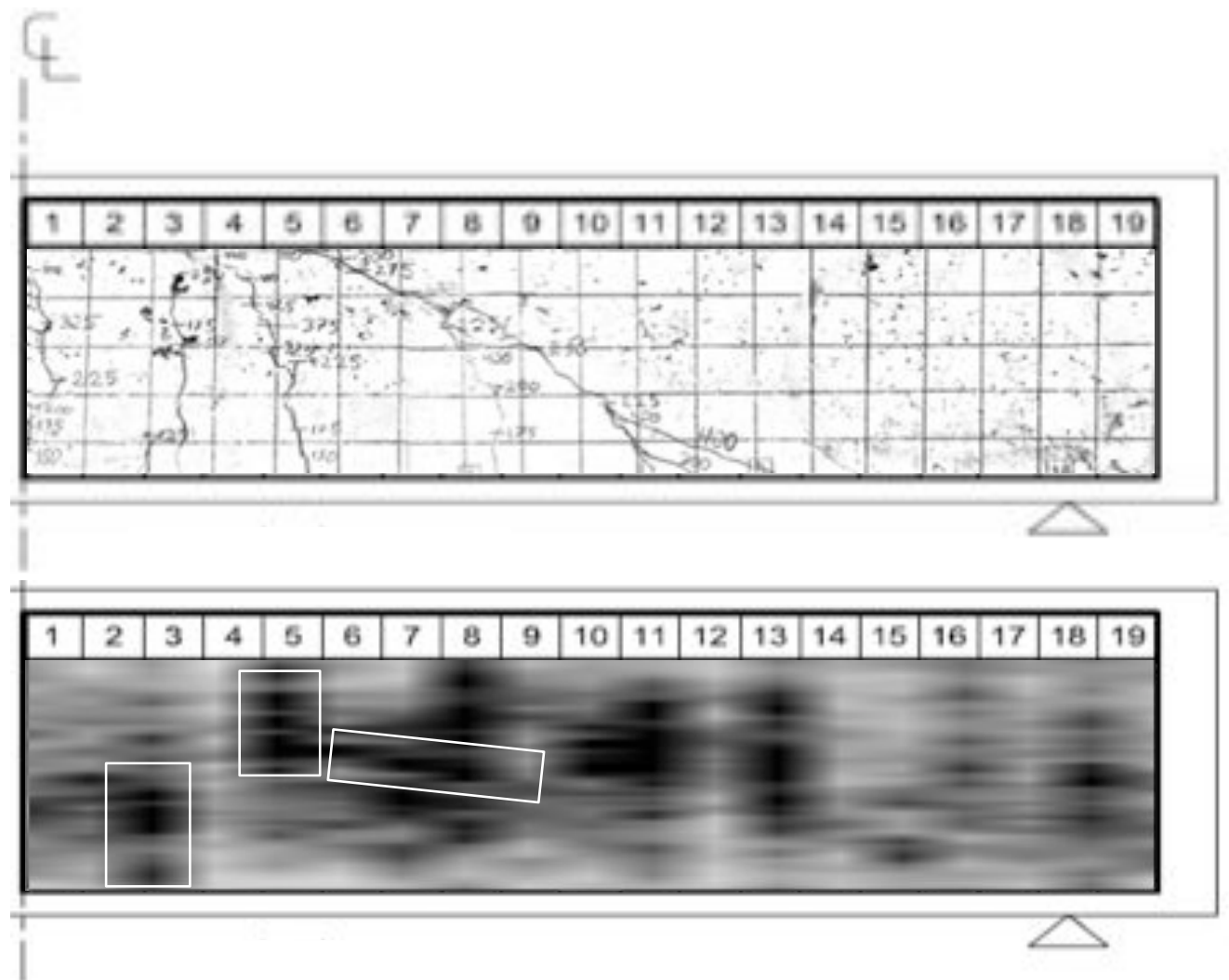

Figure 6.24: Vertical Scan Direction Beam HS-L-M-1 Damaged State; (a) Observed Cracking Pattern on Scanned Surface; (b) MIRA Software Output

The results obtained from scanning the beam, in its healthy state, in the horizontal scan direction using the MIRA tomographer are presented in Figure 6.25b, along with the 
idealized location of the steel reinforcement of the member (Figure 6.25a). This is the reference image for this scan session and provides a very clear image of the location of the steel reinforcing bars within the concrete matrix. The four black rectangles illustrate in Figure $6.25 \mathrm{~b}$ demonstrate the location of the reinforcement bars as to highlight the reflections obtained from these, and eliminate their contribution once the damaged state is analyzed for crack determination. Using the horizontal scan direction the MIRA was successful at locating the steel reinforcement very effectively in beam HS-L-M-1, and provided a clear representation of the placement condition of the reinforcement cage.

a)

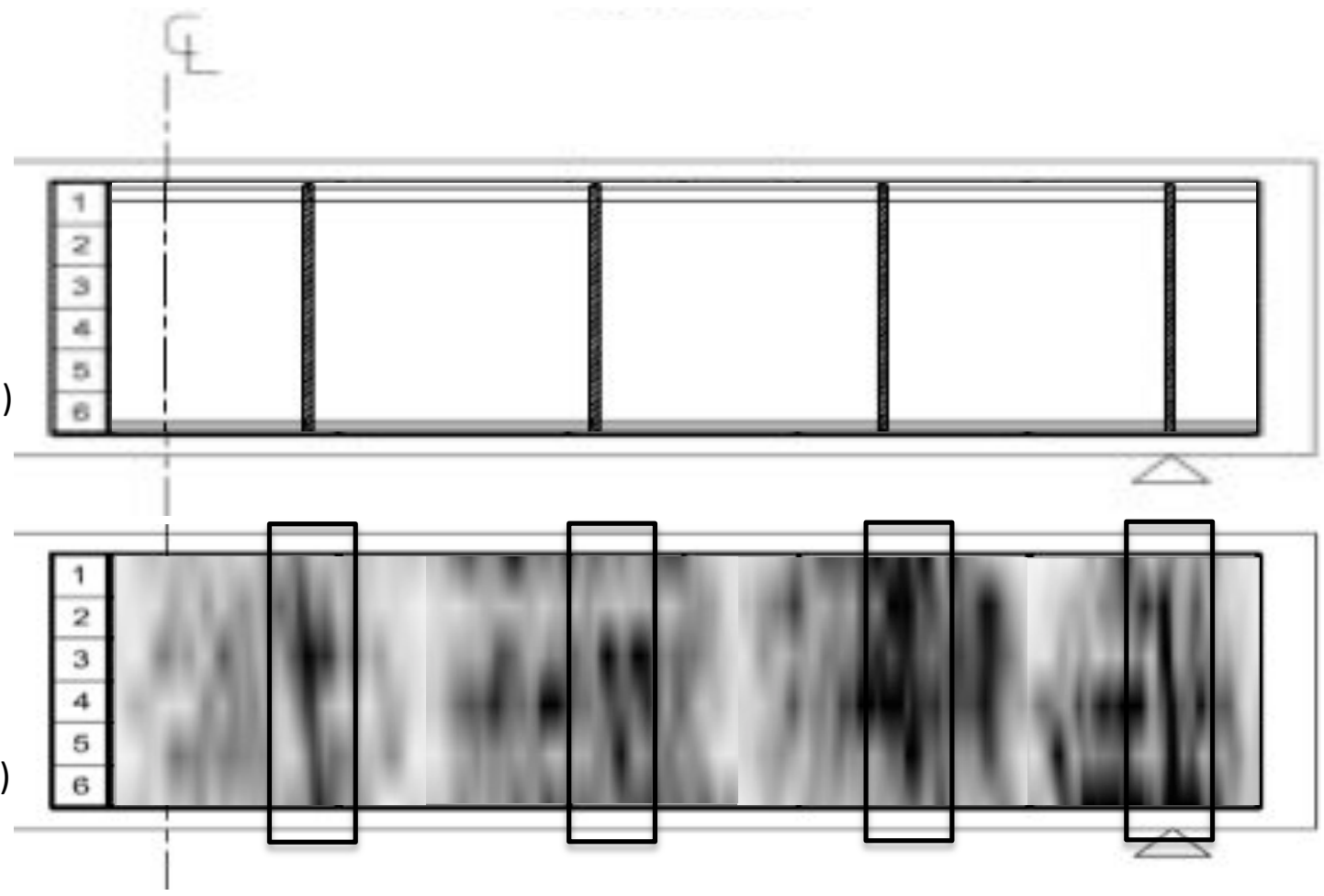

Figure 6.25: Horizontal Scan Direction Beam HS-L-M-1 Healthy State; (a) Theoretical Location of Steel Reinforcement; (b) MIRA Software Output

Figure 6.26 illustrates the observed cracking behavior of beam HS-L-M-1 (Figure 6.26a) and the respective MIRA software output of the scanned section (Figure 6.26b). The results obtained provide a good means of establishing the cracked condition of the member. By comparing the results obtained from the healthy state of the beam (Figure 6.25b), and 
those obtained from a damaged section (Figure 6.26b), several distinctions can be drawn. On the damaged state, reflections created by the steel reinforcement have almost disappeared. The high reflection of signal caused by the cracked member, attenuated the signal almost completely from reaching and reflection off of the steel bars. The concentration of signal reflection has also shifted form near the support, from the healthy state, to the quarter-span of the beam at the damaged state of the beam. This high signal reflection at the quarter-span is indicative of the damaged section as the beam was loaded to its failure load. The white rectangles illustrate the locations of cracking as illustrated by the tomographer. Although the MIRA is capable of detecting the appearance of cracking in the concrete member, the quantity of damage cannot be differentiated easily, and further assessment of the member would need to be carried out.

a)

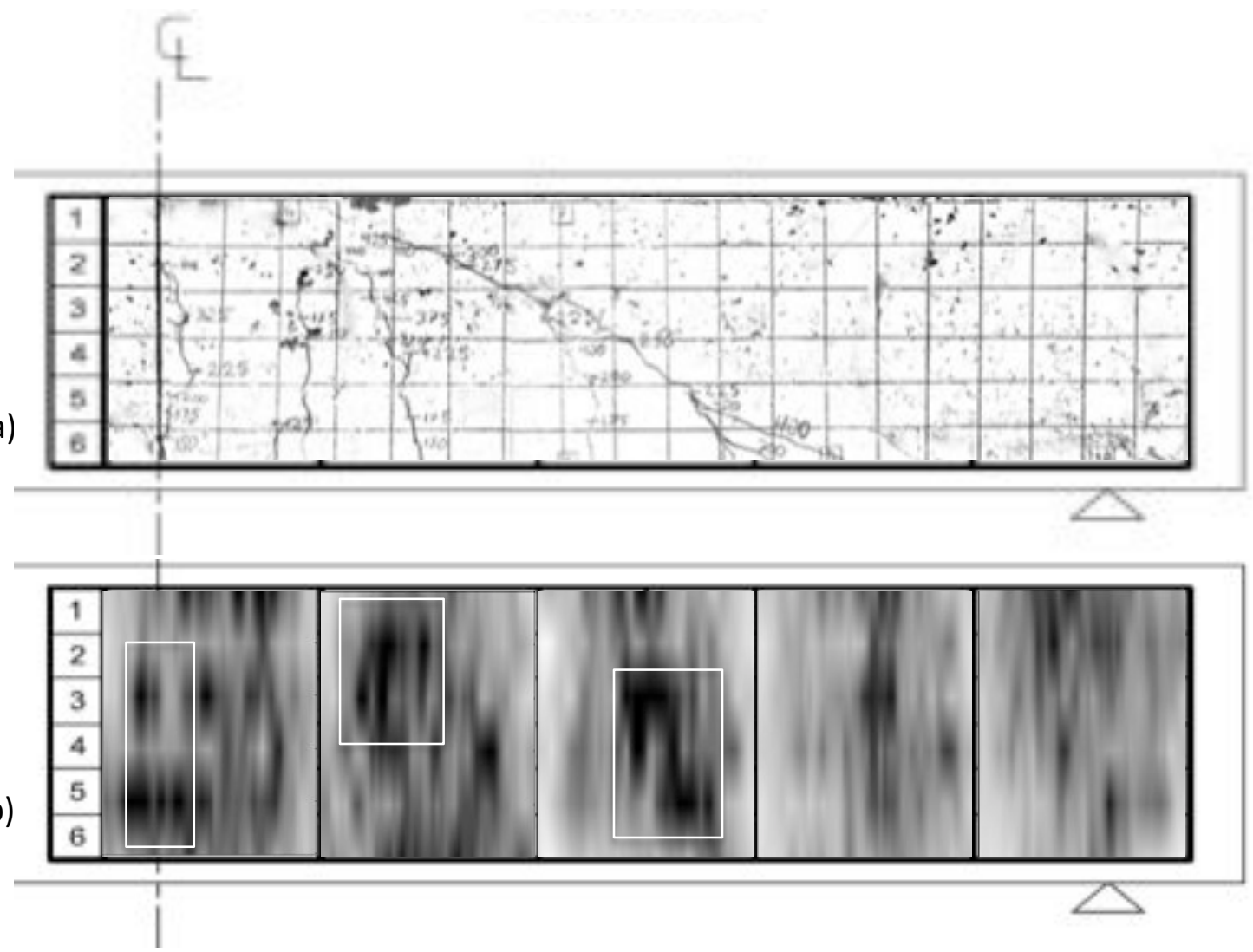

Figure 6.26: Horizontal Scan Direction Beam HS-L-M-1 Damaged State; (a) Observed Cracking Pattern on Scanned Surface; (b) MIRA Software Output 


\subsubsection{HS-L-M-2}

Beam HS-L-M-2 was designed to fail in flexure and was provided with the required shear reinforcement depicted by the code. The beam was provided with more than the minimum stirrups required, as to guarantee the desired flexural behavior of the member. The design requirements for beam HS-L-M-2 would aid in the investigation of the piezo-sensors under normal working conditions. The results for the piezo-sensors, installed in this member, were processed in terms of the strain experienced at certain locations, along with the crack-width once the member had cracked. A total of ten piezo-sensors were installed in beam HS-L-M2 , with the primary focus of investigating the flexural behavior of the beam. A total of seven signal paths were employed to monitor the flexural behavior of beam HS-L-M-2. These seven signal paths monitored the entire clear span of the beam near the tension reinforcement. An additional two signal paths were chosen to monitor the initiation of shear cracking in the member.

A five sensor multiplexed FBG fiber optic sensor was embedded into the beam's matrix for monitoring the strains at predetermined locations. Two sensors were installed at the expected shear crack locations, beside the right and left supports, and three sensors were installed at the quarter-span and mid-span. The fiber optic cables provided a comparison to the results of the piezo-system, and provided a means of verifying the piezo-system's functionality. The results of the piezo-sensors were then analyzed for a means of determining the various design limit states, as to quantify the severity of the damage experienced in terms of the code's previsions.

\subsubsection{Member Behavior}

The load deflection curves for beam HS-L-M-2 are presented in Figure 6.27. A total of three LPDTs were installed to measure the deflection of the beam during the experimental program. One LPDT was installed at the mid-span of the beam, and one at each quarterspan. From the Load-Deflection graph, member HS-L-M-2 is seen to have a failure load of 
$550 \mathrm{kN}$, at a mid-span deflection of $18.45 \mathrm{~mm}$. The quarter-span deflections were, $8.43 \mathrm{~mm}$ and $9.94 \mathrm{~mm}$, for the right and left half respectively.

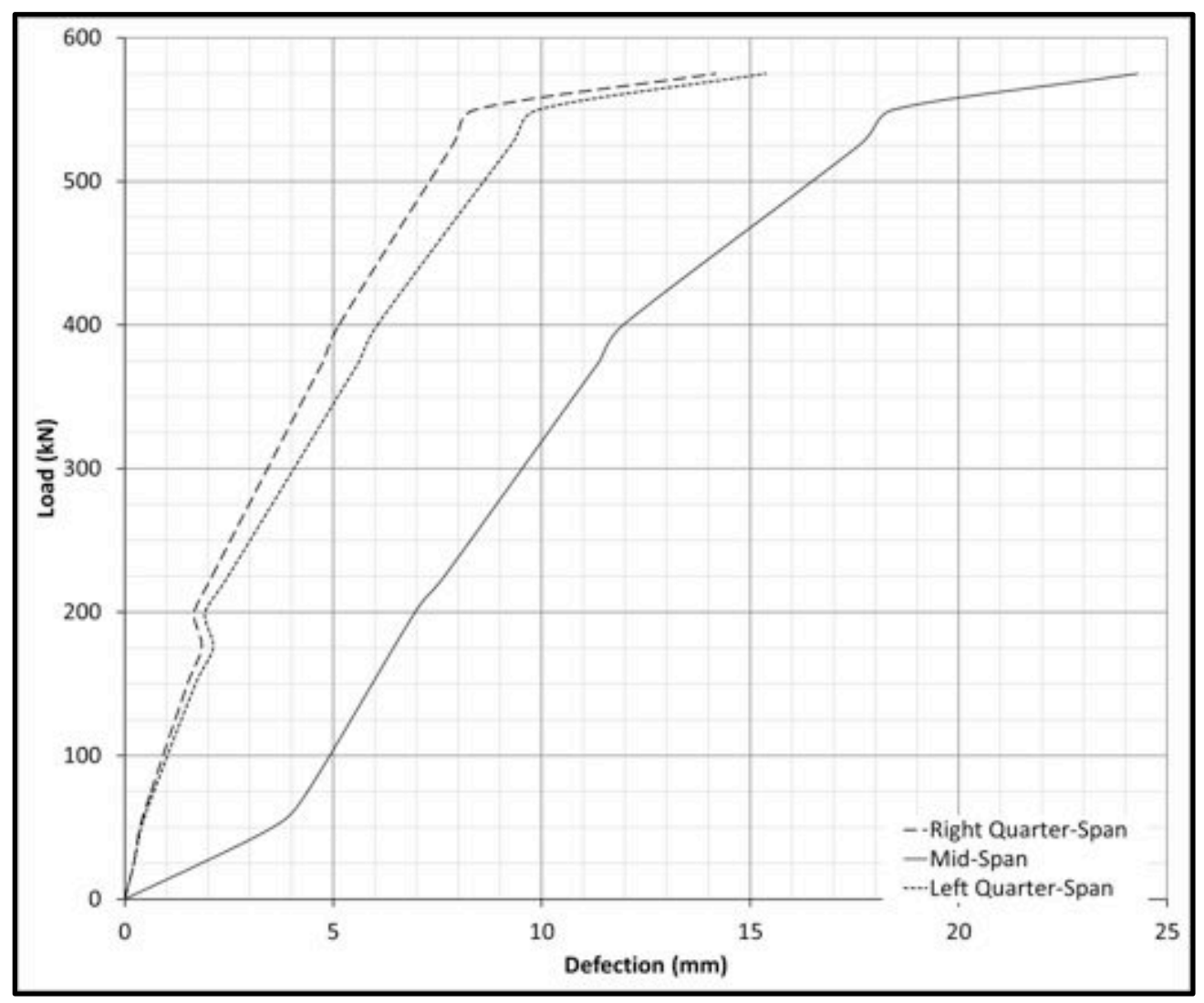

Figure 6.27: Load Defection Curve Beam HS-L-M-2

The cracking load for this member was visually observed at $150 \mathrm{kN}$, agreeing with the calculated load of $167.30 \mathrm{kN}$. The deflection experienced by the mid-section of the beam was found to be almost double of the deflections obtained from the quarter-span LPDT, as expected by the characteristic behavior of a beam exhibiting flexural behavior. The calculated Limit States for the beam were calculated to be: 326.70 kN (Serviceability), and $569.10 \mathrm{kN}$ (Ultimate). The two limit states, along with the cracking load and factored moment of resistance values were investigated, as to provide a means of determining the member's condition primarily with the aid of the piezo-system. The desired outcome of this experimental program would show that the four design limits (Cracking, Serviceability, Factored Resistance, Ultimate) for the member could be differentiated solely based on strategic analysis of the piezo-system eliminating the need for further investigations. 


\subsubsection{Piezo-Sensors Location and Results}

The ten piezo-sensors in beam HS-L-M-2 were arranged as to monitor the initiation of flexural cracking, along the tension reinforcement. The piezo-sensors' spatial position is presented in Figure 6.28. Sensors 1 through 8 monitored the tension side of the beam; while sensors 9 and 10 were used for shear crack detection. The location of the piezosensors is symmetric for the right and left halves of the beam, with the exception of the shear cracks detection signal paths. Sensor path 9-3 is located $800 \mathrm{~mm}$ from the left support while sensor pair 10-6 is located $1000 \mathrm{~mm}$ from the right support. The lack of symmetry between sensor pairs 9-3 and 10-6 would ensure the detection of the shear crack's propagation. Along with ensuring the detection of the shear crack propagation, asymmetric characteristic of the piezo-sensors also allowed for the study of varying sensor-to-sensor distances, as to establish the largest sensor separation whiles maintaining the system's reliability.

As in the investigation carried out for beam HS-L-M-1, sensor pairs 1-2 and 7-8 monitored the location near the support location. The regions are not expected to exhibit any signs of cracking, and thus, the sensors act as a control for the experiment, verifying the cracking threshold for the HMI. The combination of adjacent sensor pairs from sensor 1 through 8 would monitor the initiation of cracking along the tension face of the beam, and will be the primary focus of the study. A total of nine signal paths were studied in the investigation of beam HS-L-M-2, and are discussed here in.

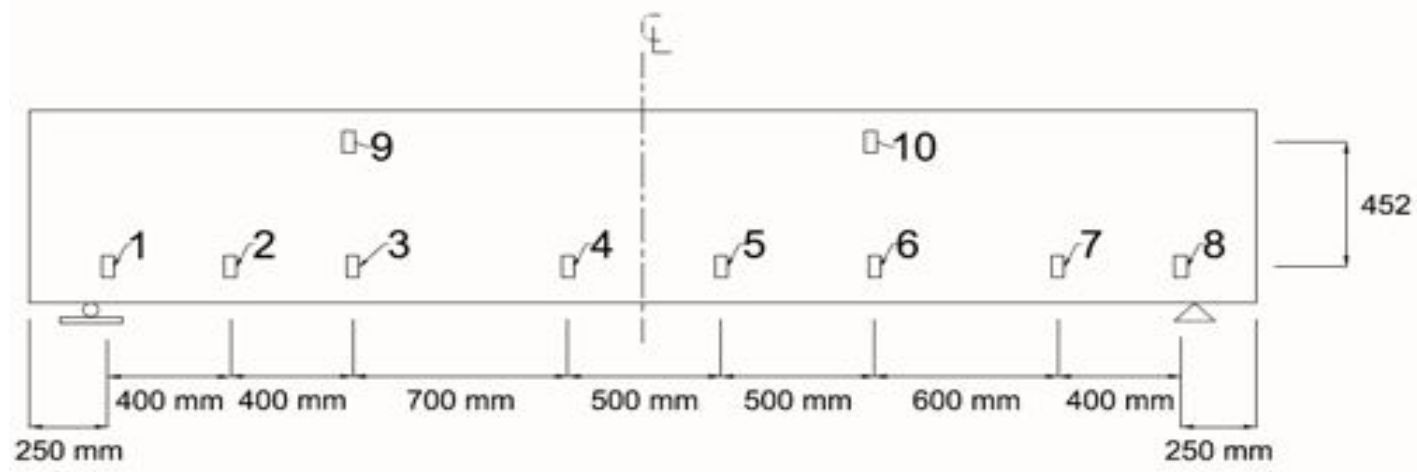

Figure 6.28: Piezo-Sensors Spatial Position in Beam HS-L-M-2 


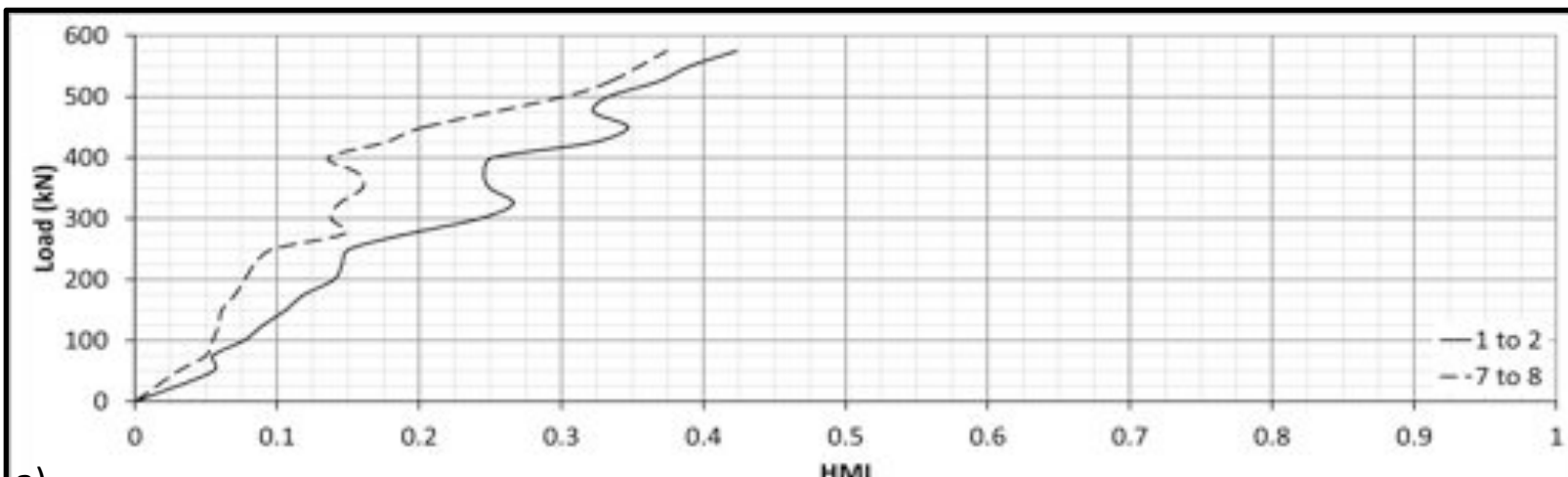

a)

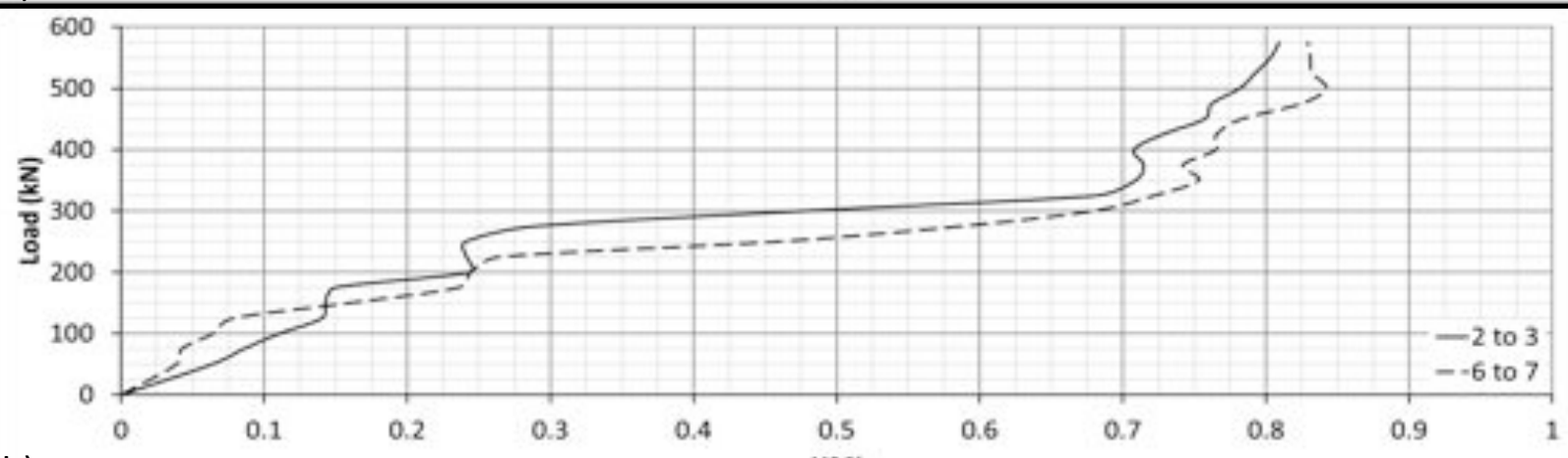

b)

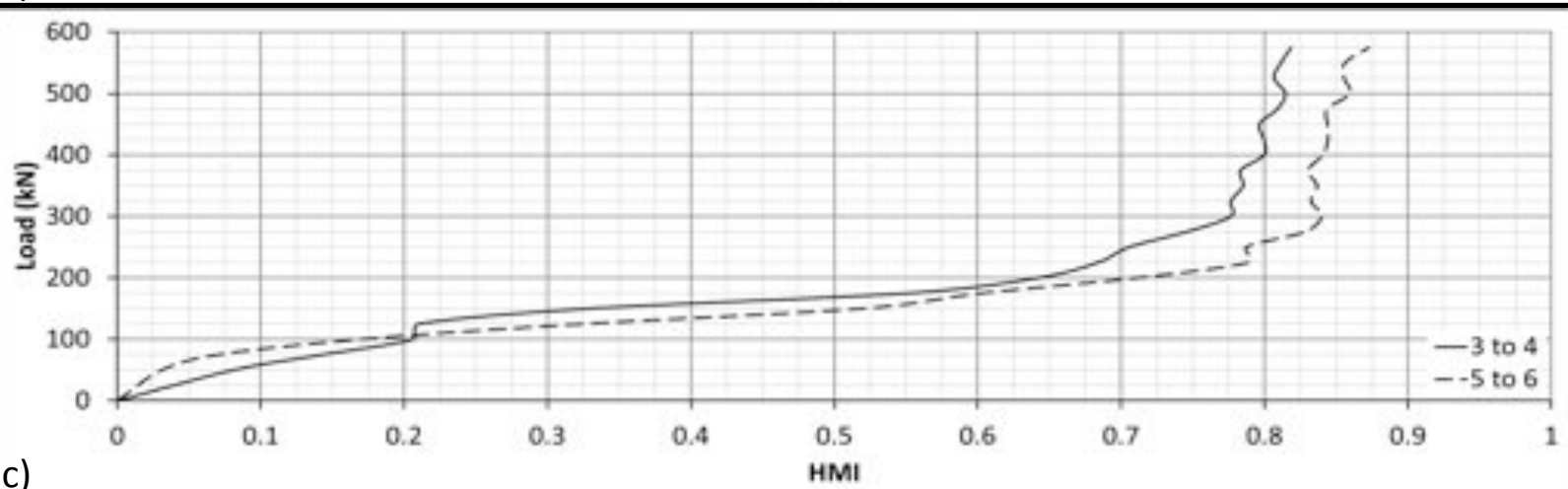

c)

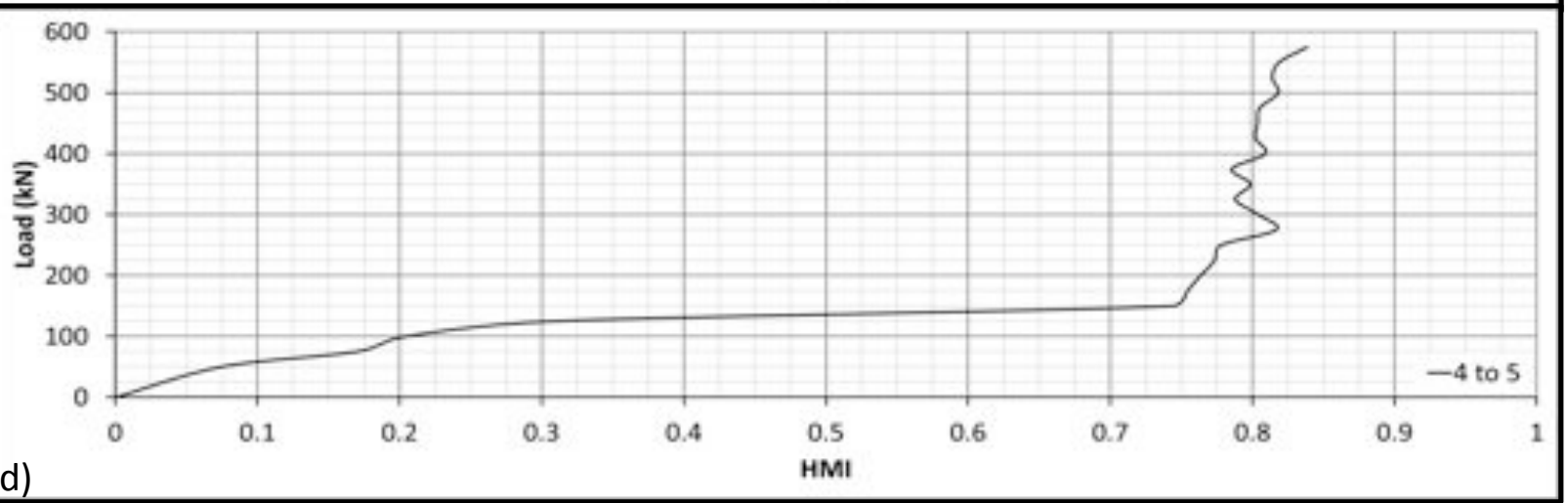

Figure 6.29: Flexural Control Load-HMI Graph for HS-L-M-2; (a) Support Location; (b) Distance dv from the Support; (c) Quarter-Span; (d) Mid-Span 
Sensor pair 4-5 monitored the mid span of beam HS-L-M-2, the Load-HMI graph for this sensor pair is presented in Figure 6.29d. Since the mid-section of the beam would experience the highest moment during the loading procedure, this location was expected to be the first to exhibit cracking. From the visual inspection of the member, a total of three cracks were observed to initiate through the signal path of the sensor pair at a load of $150 \mathrm{kN}$, yielding a $\mathrm{HMI}$ output of 0.74 . This large spike in the HMI value is characteristic of the initiation of cracking through the signal path. Although the HMI output, would suggest that the member initiate cracking at a load of $125 \mathrm{kN}$.

Sensor pairs 3-4 and 5-6 monitored the sections adjacent to the midsection of the beam. The results of the two sensor pairs are provided in Figure 6.29c. The initiation of cracking was visually observed at three load steps between sensors 3 and 5. The $175 \mathrm{kN}, 200 \mathrm{kN}$, and $250 \mathrm{kN}$ load steps exhibited signs of cracking, and had a HMI value of $0.55,0.64$, and 0.71 , respectively. The equivalent section between sensors 5 and 6 exhibited cracking at two load steps. The beam was observed to crack at the $150 \mathrm{kN}$, and $200 \mathrm{kN}$ load steps, having HMls of 0.52 , and 0.71 , respectively. Based on the results obtained, the piezo-sensors are seen to yield similar results on both ends of a symmetric beam. Although the HMIs at the cracking loads are very similar for both sensor pairs, the section between sensor pair 5-6 exhibits more distress based on the HMI output. As the load is increased beyond the cracking limit, the value of the HMI between sensors 5 and 6 becomes much greater than in the section monitored by sensor pair 3-4. Since the reflection and refraction of mechanical stress waves is largely governed by the width of the imperfection, or crack, intersecting the transmission, the results suggest that the combined crack width experienced in the section between sensors 5 and 6 is slightly larger than the one between sensors 3 and 4 .

Sensor pairs 2-3, and 6-7 monitored the sections at the quarter spans of the beam. These sections are expected to crack at a much later stage in the loading program and should be reflected by the HMI results in Figure 6.29b. Surface opening cracks were observed at two load steps between sensor pair 2-3. Cracking between this sensor pair was observed at the $325 \mathrm{kN}$ and $475 \mathrm{kN}$ load steps, yielding HMls values of 0.68 , and 0.76 , respectively. Although it should be noted that the HMI value for this section was very small up to the $275 \mathrm{kN}$ load 
step, as not much damage was incurred, after which the HMI is seen to increase tremendously as the cracking load is reached. The opposing side of the beam monitored by sensor pair 6-7 showed similar attributes. The HMI value was under 0.3 as the load was approaching $225 \mathrm{kN}$, although as the load was increased further, approaching the cracking load of $275 \mathrm{kN}$ the $\mathrm{HMI}$ value reached 0.59 . The second observed crack was at a load of 375 $\mathrm{kN}$, increasing the HMI value to 0.74 . Overall both pairs of sensors yielded very similar results, although as discovered with sensor pairs 6-7, the right side of the beam shows much higher HMI values and thus would have a higher combined crack width. Based on the results of the $\mathrm{HMI}$ value and the threshold developed in the small scale experimental program, the region between sensors 2-3 would have been considered cracked at a load of $300 \mathrm{kN}$. Similarly the region between sensor pair 6-7 would have been considered cracked at a load of $250 \mathrm{kN}$. Thus the results of the piezo-system provide the means of early crack detection; providing the initiation of cracking within a member $25 \mathrm{kN}$ prior to the damage becoming visible at the exterior.

Sensor pairs 1-2 and 7-8 monitored the sections directly adjacent the supports. These sections were not expected to crack, but rather to act as a control for the experiment. The $\mathrm{HMI}$ was calculated, as it was very useful in validating the previously established benchmark for the HMI. As the support sections between sensors pairs 1-2, and 7-8, had maximum HMIs of 0.42 and 0.38 , it can be concluded that 0.5 is an acceptable limit as to differentiate between a section where cracking is visible at the beam's surface exterior, and a section that is not exhibiting signs of cracking.

The appearance of the shear cracks in beam HS-L-M-2 was monitored by sensor pairs 9-3 and 10-6, the results of which are shown in Figure 6.30. The 9-3 signal path was visually observed to be intercepted by a shear crack at the $325 \mathrm{kN}$ load step. At the same load the $\mathrm{HMI}$ value output by the piezo-sensors surpassed the cracking threshold of 0.5. Sensor pair 10-6 was located $200 \mathrm{~mm}$ closer to the center of the beam than sensor pair 9-3. This shift in sensor location resulted in the sensors not capturing the shear crack, but rather, the sensors pair, captured the initiation of a flexural-shear crack. The crack intercepted the signal transmission at a load of $300 \mathrm{kN}$, yielding an $\mathrm{HMl}$ value of 0.51 . These results indicate that 
the sensor pairs, although measuring different cracking mechanisms were successful in capturing the inflicted damage. Both piezo-sensor pairs yielded similar results, and had they been the primary method for judging the member's condition, the piezo-system would have demonstrated a clear warning for the initiation of the shear crack.

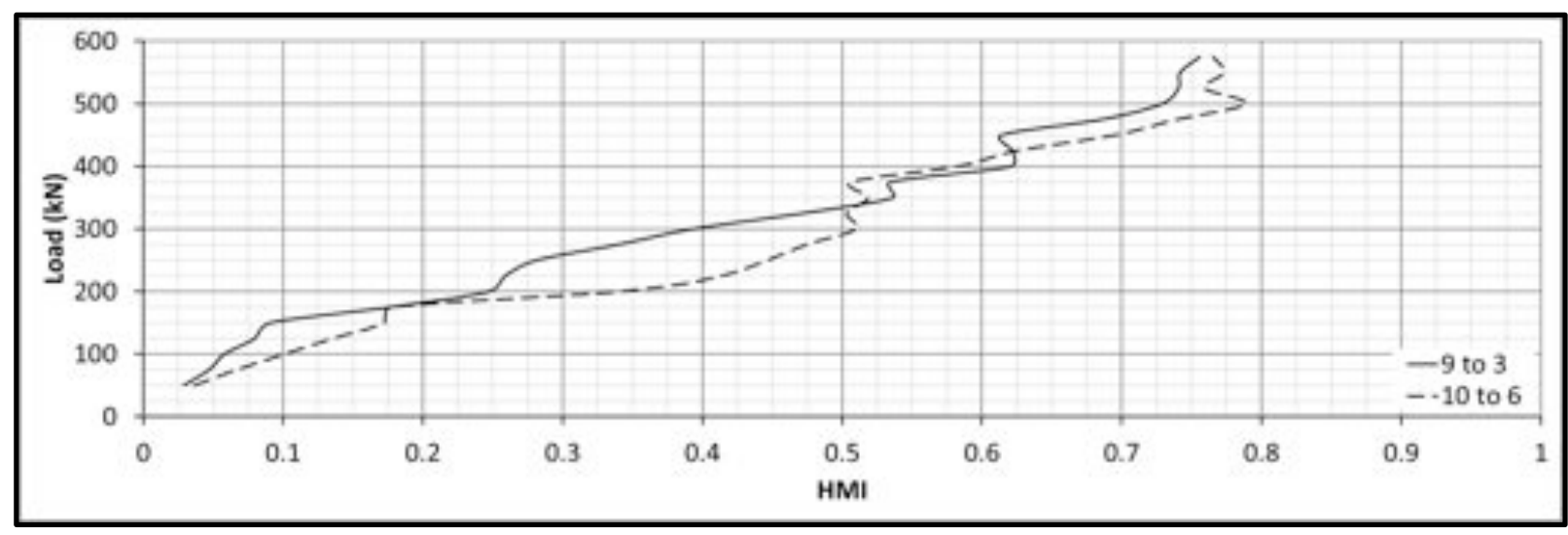

Figure 6.30: Shear Crack Detection

\subsubsection{FGB Fiber Optic Readings}

A total of 5 FBG fiber optic sensors were installed in beam HS-L-M-2. The sensors were multiplexed onto one cable that was installed to measure the strain at the shear crack location, at the quarter-span of the beam, and at the mid-span. The sensors were intended to provide a comparison between the crack width measurements provided by the piezosensors, and the direct measurements taken by the well-established fiber optic system. The fiber optic cable was installed exactly as in beam HS-L-M-1. Again, due to the high sensitivity of the cable, only three of the five sensors provided readings for this beam. The cable is thought to have ripped during the casting process just after the third sensor location. The working sensors' locations are illustrated in Figure 6.31a

The results obtained from the fiber optic cable represent the right half of beam HS-L-M-2. The sensors provide readings of the strain across the shear crack, the quarter-span location and the mid-span location of the beam. By adopting the symmetry of the beam, the results obtained from the quarter-span and shear crack location can be used to approximate the behavior of the left half of beam HS-L-M-2. The mid-span fiber optic sensor provides 
reading that are in direct comparison with sensor pair 4-5, where the quarter span fiber optic sensor provides a direct comparison to the results obtained from sensor pairs 6-7 and 3-4. The shear sensor will be compared to the results obtained from the sensor pair 10-6 as well as sensor pair 9-3, employing the beam's symmetry.

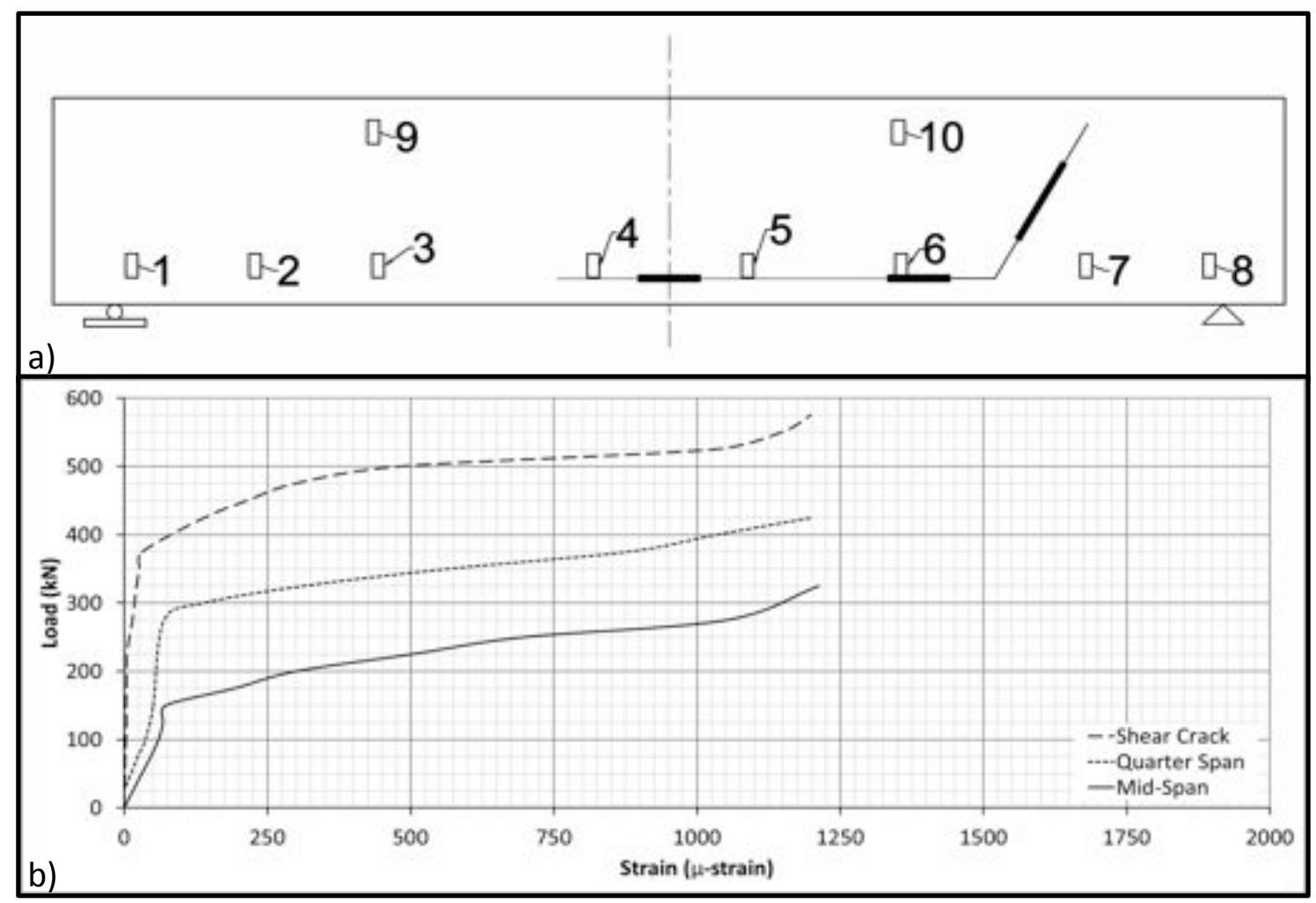

Figure 6.31: FBG fiber optic readings from beam HS-L-M-2; (a) Location of working sensors; (b) Readings obtained from FBG Sensors

The results obtained from the mid-span fiber optic sensor show that the region exceeded the $110 \mu$-strain limit at a load of $150 \mathrm{kN}$. The sensor's results agree with the visually observed cracking load of $150 \mathrm{kN}$ and the calculated $167 \mathrm{kN}$ load. The results of the piezosensors yielded a cracking load of $125 \mathrm{kN}$, forecasting the initiation of cracking. The sensor provided accurate strain reading up to a value of $1200 \mu$-strain at the $325 \mathrm{kN}$ load step. The quarter-span sensor exceeded the $110 \mu$-strain at the $275 \mathrm{kN}$ load step, agreeing with the visually observed pattern, although differing slightly from the piezo-system that yielded the cracking load of this region to be $250 \mathrm{kN}$. The shear crack sensor captured the occurrence of the shear crack at the $400 \mathrm{kN}$ load step, agreeing with the observed cracking load. Although 
no comparison can be drawn between the fiber optic sensor and sensor pair 10-6, due to the fact that the shear crack never intersected the signal path. The sensor then provides an indirect comparison of the member's behavior to the region measured by the piezo-sensor. Both the quarter-span, and shear crack sensor were effective up to a strain of $1200 \mu$-strain, after which they exceeded their range.

\subsubsection{Strain Readings Using Proposed Model}

The small scale experimental program concluded with the development of a HMI-Strain relationship representing the internal strain experienced by a member during the uncracked stages of the member's lifetime. The developed model was applied to the HMI results obtained by each of the seven signal paths that monitored the flexural behavior of beam HS-L-M-2. The results of the fiber optic sensors, where available, are also presented parallel to the piezo-sensor results, as to provide a means for comparison of the accuracy of the piezoceramic system. The results of the investigation are presented in Figure 6.32. The strain readings provided by the piezo-sensors can also be compared to the observed cracking pattern of the beam as to validate the cracking limit for the HMI.

The most critical section for monitoring the beam's strain is the location along the span, directly adjacent to the support location. The location on either side of the member is not expected to crack until the beam's capacity is exceeded, and the member had reached the failure stage. As the effective shear depth of the specific member was $505 \mathrm{~mm}$, the beam is not expected to exhibit any cracking up to a distance exceeding $505 \mathrm{~mm}$ from the support location. Sensor pairs 1-2 on the left side, and sensor pair 7-8 on the right side of the member monitored this specific region. The results for the internal strain of the regions, as calculated from the HMI results, is presented in Figure 6.32a. These results show that beam HS-L-M-2 experienced a much higher concentration of stress at the left support. From the readings obtained between sensor pair $1-2$, the beam exceeded the $110 \mu$-strain limit at a load of $450 \mathrm{kN}$, suggesting the initiation of cracking. Based on the results of the piezosensors, the region between sensor pair 1-2 is witnessed to have a final strain reading of 
$200 \mu$-strain at the failure load of $575 \mathrm{kN}$. The equivalent crack width for this region is calculated to be $0.036 \mathrm{~mm}$, suggesting the crack is too small to be visible with the naked eye, and thus the reason for it not being visually identified. The right half of the beam, between sensor pair 7-8, showed a slightly less severe amount of damage. The strain in this region remains within the un-cracked limit up to the load step of $550 \mathrm{kN}$, demonstrating the majority of strain increase in between the 450 and $575 \mathrm{kN}$ load steps. The maximum value for strain reached in this region was of $135 \mu$-strain. Overall the sensor pairs monitoring the location adjacent to the supports proved to be rather reliable during the testing period. The sensors showed minor changes in the strain of the region in the early stages of loading, and demonstrated a clear and consistent appearance of hairline cracking after the beam's factored resistant moment was exceeded, just as expected of the member's behavior.
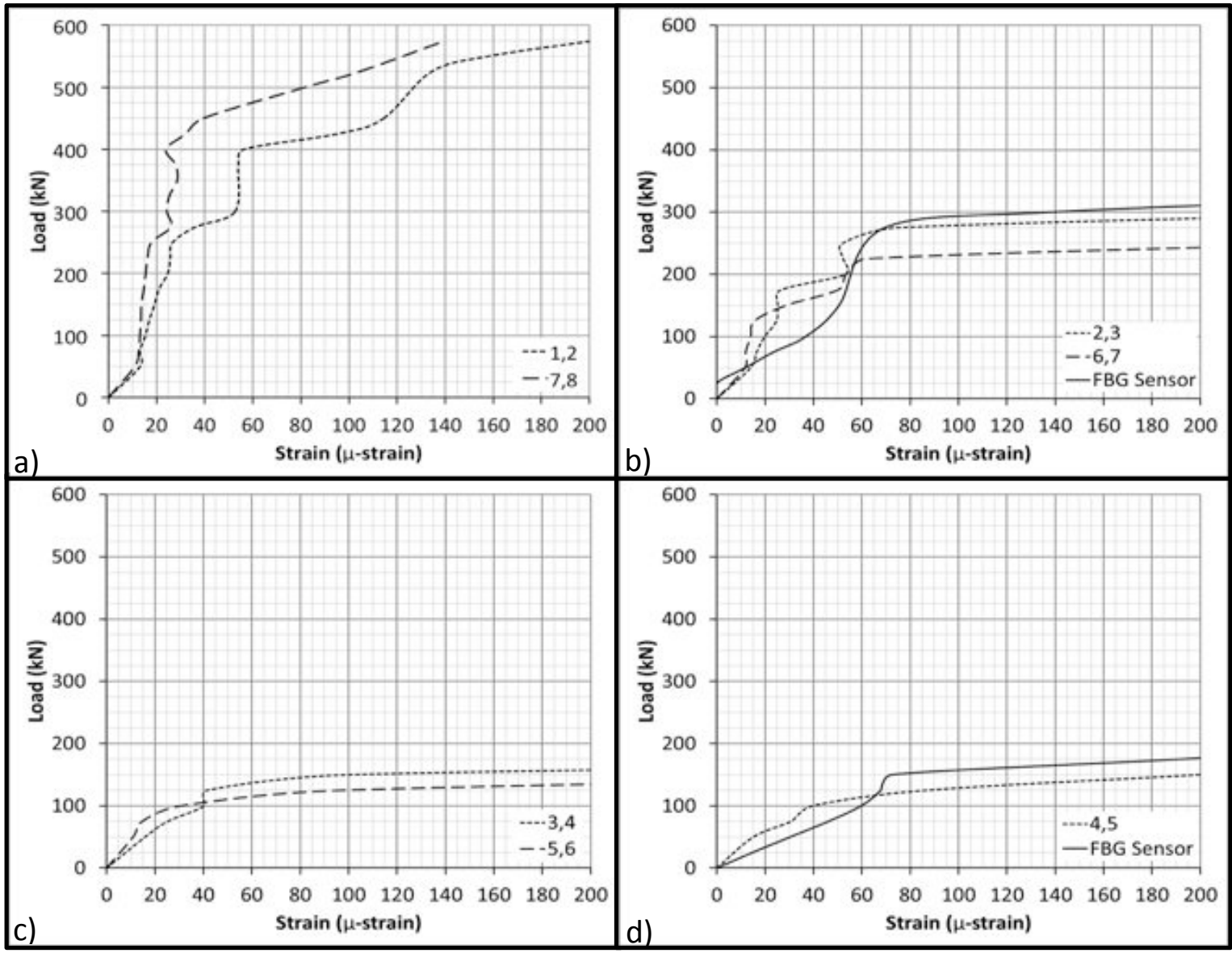

Figure 6.32: Strain Reading Obtained from Beam HS-L-M-1; (a) Adjacent to Support Location; (b) Quarter Span Location; (c) Adjacent to Mid-Span Location; (d) Mid-Span Location 
Sensor pairs 4-5 monitor the signal path of the mid-section of the beam, along with a FBG fiber optic cable. The strain readings obtained from each system are presented in Figure $6.32 \mathrm{~d}$. From the presented results the two systems are observed to provide very similar results. The primary difference being the monitored distance of each system, where the fiber optic sensor is monitoring $200 \mathrm{~mm}$ of the beam the piezo-ceramic sensors are monitoring $500 \mathrm{~mm}$. The results of the piezo-sensors are thus expected to yield slightly higher values of strain than the fiber optic gauge, although the two systems should follow the same trend, just as illustrated by the results obtained.

The quarter-span and section adjacent to the mid-section are presented in Figure 6.32b, and Figure 6.32c. Sensor pair 2-3 on the left side of the beam, and sensor pair 6-7 along with a fiber optic cable monitored the right side of the beam. From Figure $6.32 \mathrm{~b}$ the results of the three reading are seen to be in good agreement with one another. The results of the piezo-sensors from the left and right halves of the beam are in good agreement with one another, where the right side of the beam illustrates a slightly higher amount of damage. The section adjacent to the mid-section monitored by sensor pairs 3-4 and 5-6 demonstrate a similar trend. From the Load-Strain graph in in Figure 6.32c, the sensor pairs demonstrate a very symmetric behavior for beam HS-L-M-2, although, consistent with the quarter-span, the strain values on the right side of the beam are seen to be slightly higher than those captured from the left. The graph for this section also illustrates a very similar behavior to the one exhibited by the mid-section of the beam. As the two sections undergo the same value of moment, the two sections are expected to have very similar behaviors during the loading stages.

The location based strain readings obtained from the piezo-system can be very beneficial for the non-destructive evaluation of a beam, as discovered from this experimental program. The piezo-sensors are very effective at capturing the initiation of cracking in a reinforced concrete member. Using this knowledge, along with the limits defining a cracked member (110 $\mu$-strain and HMI exceeding 0.27) can provide a means of establishing the various stages defined by the code that a member undergoes during its loading program. The experimental data obtained illustrated a clear representation of the two loading stages: 
the cracking load, and the ultimate resistant moment. As the piezo-sensors are very sensitive to the appearance of cracking, the cracking load is easily captured by the sensors monitoring the location of maximum moment in the member, in this case sensor pair 4-5. The calculated cracking load, based on the Canadian code previsions, was found to be 167.3 $\mathrm{kN}$ for the specified loading set up. The initiation cracking in beam HS-L-M-2 was observed $17 \mathrm{kN}$ earlier at a load step of $150 \mathrm{kN}$, suggesting the member cracked before this load step. Based on the obtained results, the piezo-sensors captured cracking at the mid-span location, as well as the locations adjacent to the mid-span of the member, at the $125 \mathrm{kN}$ load step.

The moment when the beam exceeded its ultimate resistance moment was captured using a slightly indirect technique. The basic behavior of a reinforced concrete beam dictates that a beam operating in its design limits will not crack within a distance, equal to the effective shear depth, from a support. Thus a crack within the region adjacent to the support would imply that the beam has exceeded its capacity, or the ultimate moment of resistance of the member. The beam's ultimate carrying capacity was calculated to be $569 \mathrm{kN}$ based on the previsions of the Canadian code. The graph illustrating the member's behavior, Figure 6.27, yields that the member's ultimate carrying capacity was, in fact, exceeded at the $550 \mathrm{kN}$. Although, no visual observation of the cracking at the support location was captured, the piezo-system demonstrated that the left side of the beam cracked in this region at a load of $550 \mathrm{kN}$ where the right side of the beam exceeded the theoretical cracking limit at $475 \mathrm{kN}$. Thus, the piezo-system was found to be effective for determining of two of the four stages of the member's loading program. The continuing objective is to successfully identify the load step at which beam HS-L-M-2 exceeded its serviceability limit.

\subsubsection{Crack Width Measurements Using Piezo-System and Member Cracking Behavior}

The HMI values provided by the piezo-sensors for the effective cracking region, along with the results obtained shear crack sensors were converted to the equivalent crack widths. The results of this are presented in the following section, and are compared to the results 
obtained from the FBG fiber optic sensor, where available. A total of five signal paths were used to monitor the effective cracking region of beam HS-L-M-2, and two signal paths to monitor the shear crack propagation.

Sensor pair 4-5 monitored the mid-section of the beam, and the results obtained from the investigation are presented in Figure 6.33. The Load-Crack Width results obtained from both the piezo-sensors and the FBG fiber optic sensor in this region are illustrated in Figure 6.33a, and the observed cracking pattern of the region is presented in Figure $6.33 \mathrm{~b}$. The cracking load for this region was observed to be $150 \mathrm{kN}$, as demonstrated by the results of the FBG fiber optic cable. The results of the piezo-system are illustrated in Figure 6.33a, along with the results of the fiber optic cable. The results of the piezo-sensors do reflect the cracking load of the beam, although the measurements of the crack width are thought to not have been effective in this case. The main concern with these readings is the large spike in $\mathrm{HMI}$ following the $125 \mathrm{kN}$ load step, and the location of the crack nearest to sensor number 5. The crack is thought to have initiated across the surface of sensor 5, compromising the bond of the sensor to the concrete matrix and consequently, skewing the results. The sensor pair is still affected by the increase in strain in the particular section, although the calibration between the $\mathrm{HMI}$ and crack width is not valid anymore. Therefore, the results of the sensor pair should be interpreted for crack detecting only.

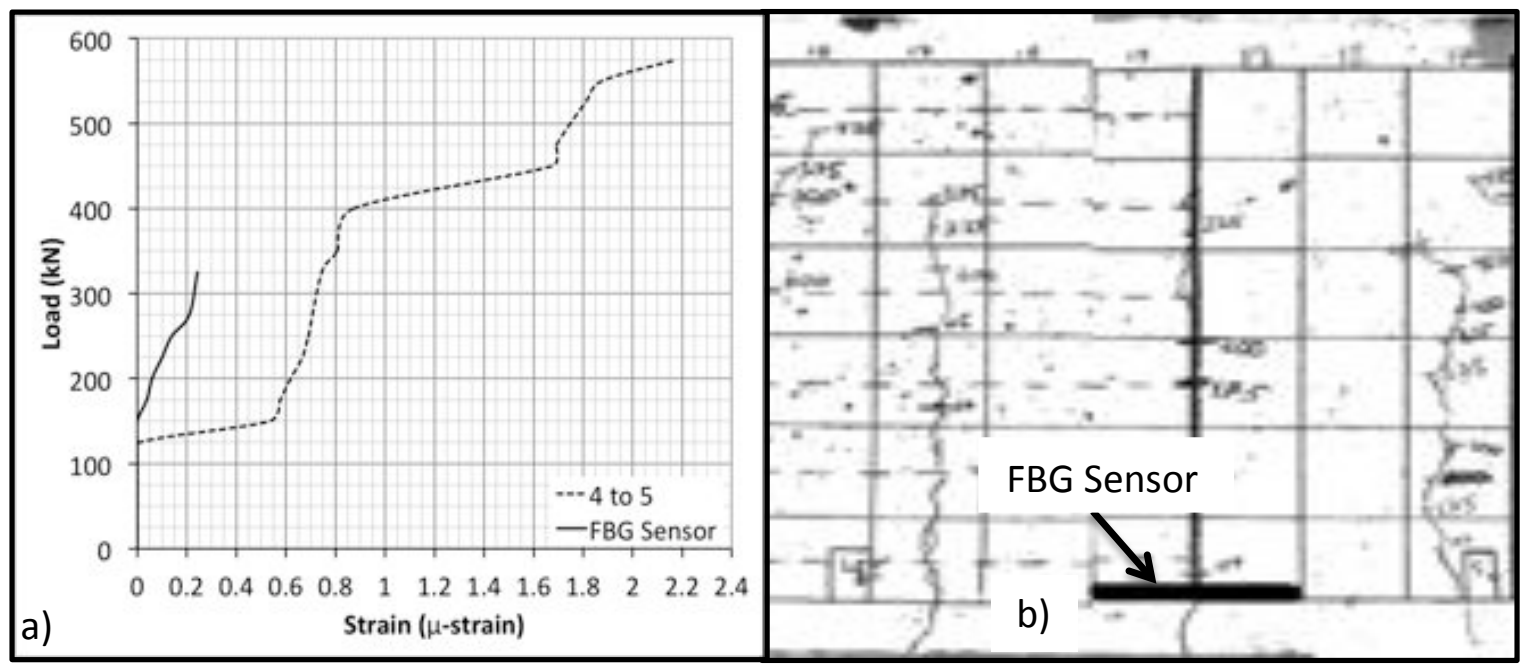

Figure 6.33: Results obtained at the Mid-Span of Beam HS-L-M-2 (a) Load-Crack Width Graph yielded by Piezo-Sensors and FBG Cable; (b) Observed cracking pattern, and location of FBG sensor 
Sensor pairs 3-4 and 5-6 monitored the sections adjacent to the mid-section of beam HS-LM-2. The results obtained from the piezo-sensors, along with the cracking behavior of the beam are illustrated in Figure 6.34. The crack width measurements from either side of the beam are seen to be in good agreement with one another, showing a slight variation between the readings. The gauge length measured by sensor pair 3-4 is $700 \mathrm{~mm}$, where the distance between sensor pair 5-6 is $500 \mathrm{~mm}$. The difference between the effective signal lengths did not cause either sensor pair to be exposed to more cracks. From the cracking behavior of the two regions the intersection of the signal path by two cracks can be seen on either side of the beam. The results confirm the symmetry of the beam, and demonstrate that the right side of the beam was slightly weaker than the left half. The results is deduced from the combined crack width of either region, where the crack width measured for the right half of the member, is slightly larger than that measured for the left half.

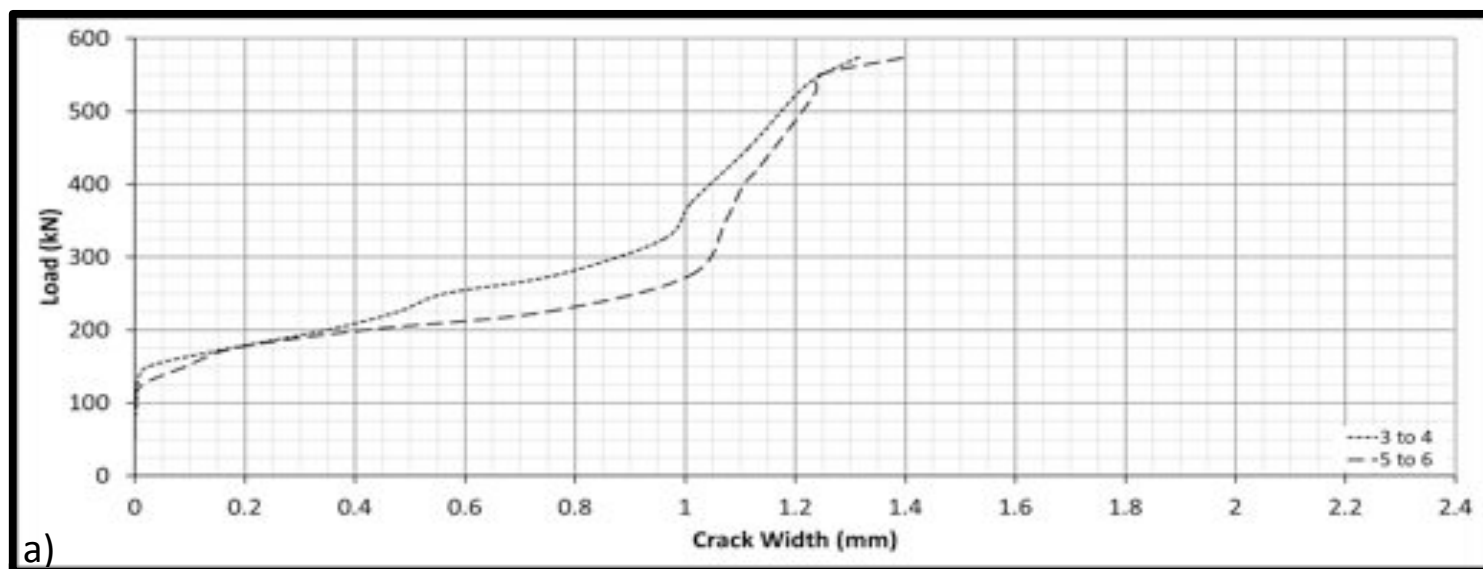

a)

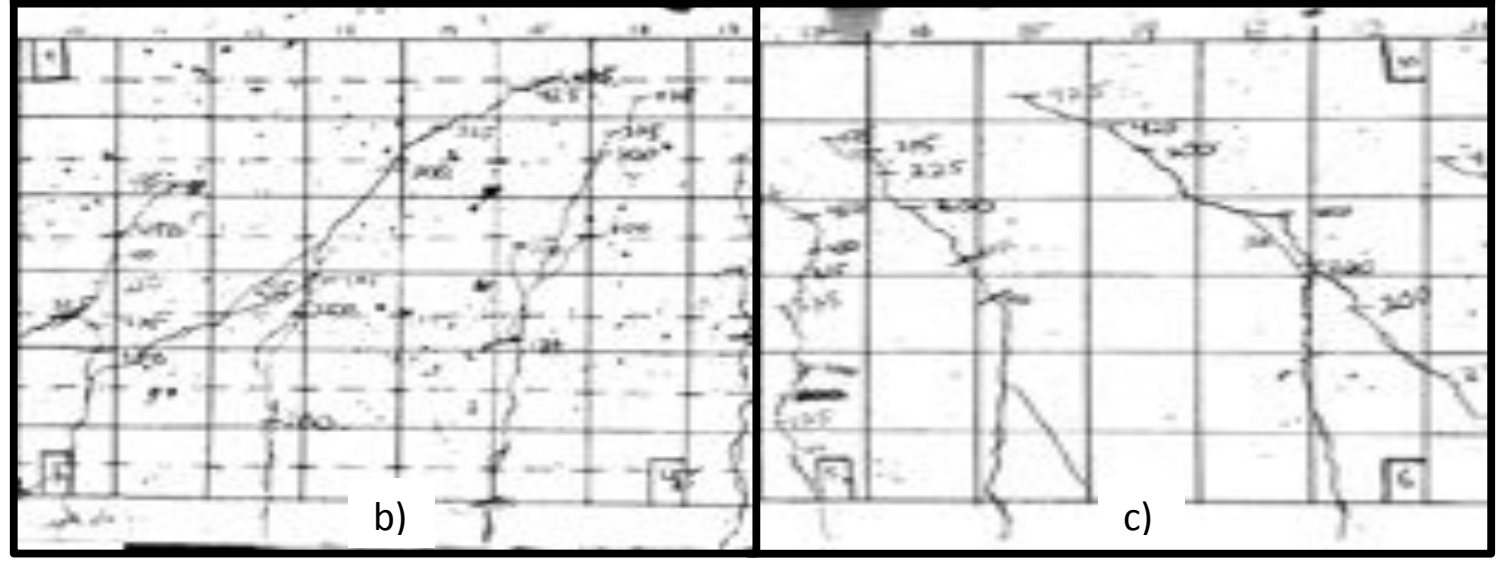

Figure 6.34: Results obtained adjacent to the support region HS-L-M-2; (a)Load-Crack Width Graph as yielded by piezo-sensors; (b) Observed cracking pattern between sensors Pair 9-3, left side of the beam; (c) observed cracking pattern between sensor pair 10-6, right side of the beam 
Sensor pairs 2-3 and 6-7 monitored the quarter-spans on either side of the member. Along with the results of the piezo-sensors, a fiber optic cable was installed between sensor pair 6-7 to monitor the strain near the tensile reinforcement. The results of the fiber optic cable, along with the measurements provided by the piezo-sensors are presented in Figure 6.35. The results of the piezo-sensors are in good agreement with the results obtained from the section adjacent to the mid-section of the member, suggesting the right side of the beam experiences a slightly higher degree of damage than the left half.

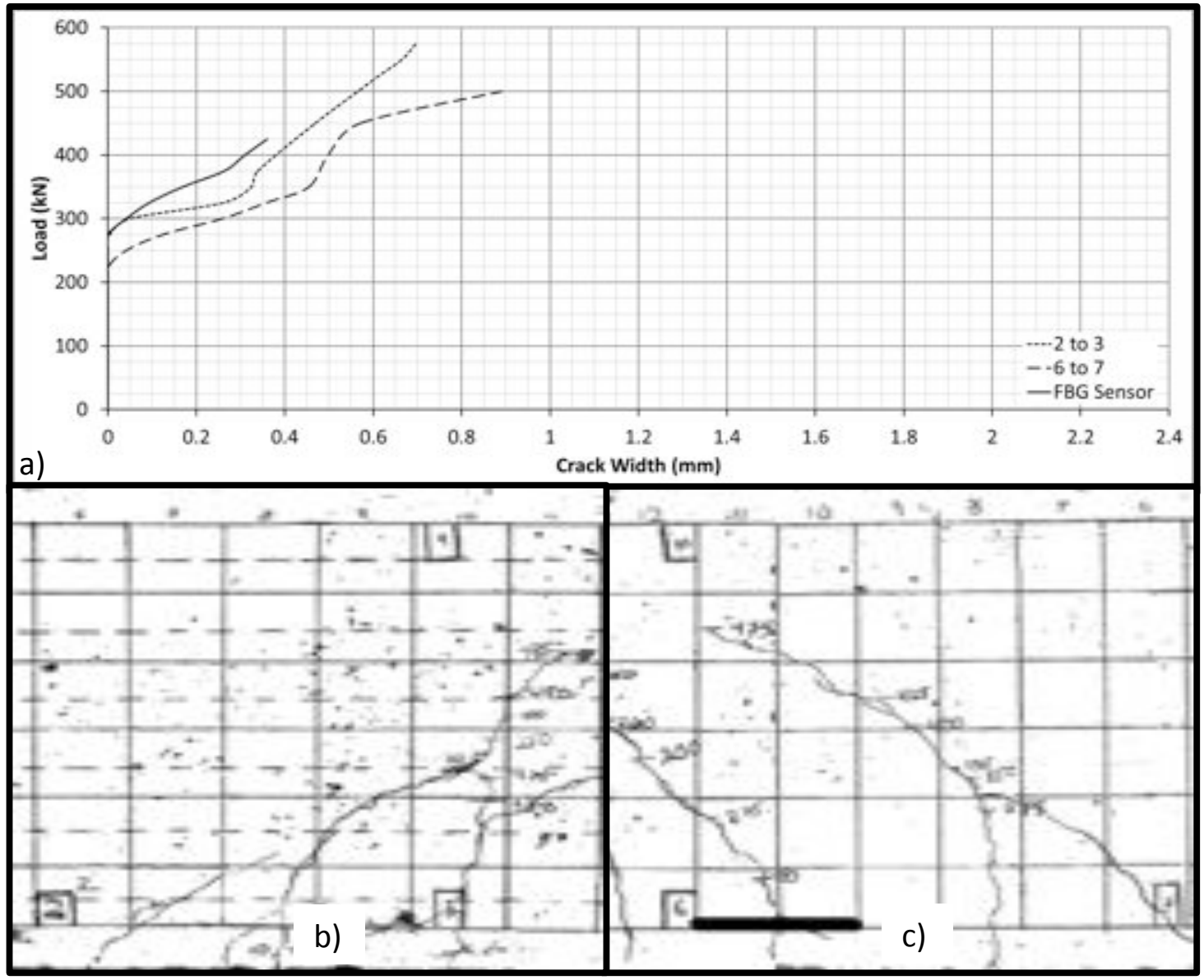

Figure 6.35: Results obtained at $d_{v}$ from the support of beam HS-L-M-2; (a) Load-Crack Width Graph yielded by Piezo-Sensors and FBG Cable; (b) Observed cracking pattern between sensors Pair 2-3, left side of the beam; (c) Observed cracking pattern between sensor Pair 6-7, right side of the beam

The Load-Crack Width graph of the two quarter-span sections can be found in Figure 6.35a along with the results obtained from the fiber optic gauge, while the cracking behavior of the left and right sides are presented in Figure 6.35b and Figure 6.35c, respectively. The 
results illustrate a strong agreement between the results of the fiber optic cable and the piezo sensors. The results of the fiber optic cable are expected to be in direct comparison to the ones obtained from sensor pair 6-7. The primary difference between these two readings is the effective gauge length of each system. The fiber optic cable had an effective gauge length of $200 \mathrm{~mm}$, while the piezo-system monitored a section that was $600 \mathrm{~mm}$ in length. Figure 6.35 illustrates the difference between the two readings, where the fiber optic system captured and measured the width of one crack, the piezo-system combined measured the width of two cracks in the region up to a load of $475 \mathrm{kN}$. The shear crack that propagated near the location of sensor seven was visually observed at the $450 \mathrm{kN}$ load step, and is reflected in the readings obtained from the piezo-sensors.

The final region monitored using the piezo-sensors system was the location of the shear crack. Since the exact location of the propagation of the shear crack was not known prior to casting and loading the member, the two sensor pairs were not symmetrically installed in the beam. Sensor pair 9-3 was located $800 \mathrm{~mm}$ from the left support, and sensor pair 10-6 was located $1000 \mathrm{~mm}$ from the right support. The results obtained for the crack width measurements of the two locations are presented in Figure 6.36a, along with the results of the fiber optic sensor for measuring the crack width. The shear crack propagation is illustrated in Figure 6.36b, for the left side of the beam, and Figure 6.36c for the right side of the member. Here the cracks intersected the signal paths of the piezo-sensors at the loads of $325 \mathrm{kN}$ for the left, and $300 \mathrm{kN}$ on the right half of the member. Although, it should be noted that the results of the fiber optic cable do not agree with those of sensors pair 106. Sensors pair 10-6 is measuring the width of a flexural shear crack where the fiber optic cable is measuring the width of the shear crack directly. The results of sensor pair 10-6 should be comparable to those of 6-7, as the two signal paths are effectively measuring the widths of the same crack, at different locations along its length. The results of sensor pairs 6-7 and 10-6 provide an interesting reflection of the beam's behavior. Based on the results, the initiation of cracking between sensor pair 10-3 propagated $25 \mathrm{kN}$ prior to the formation of a crack between sensor pair 6-7. The result would yield that the crack monitored by sensor pair 10-6 originated as a shear crack, at half of the beam's height, after which the 
crack intersected a flexural crack initiating from the tension face of the beam. The width between sensor pair 6-7 is seen to increase at a much higher rate than further along this crack, this is to be expected due to the varying strain gradient from the top to the bottom of the beam.

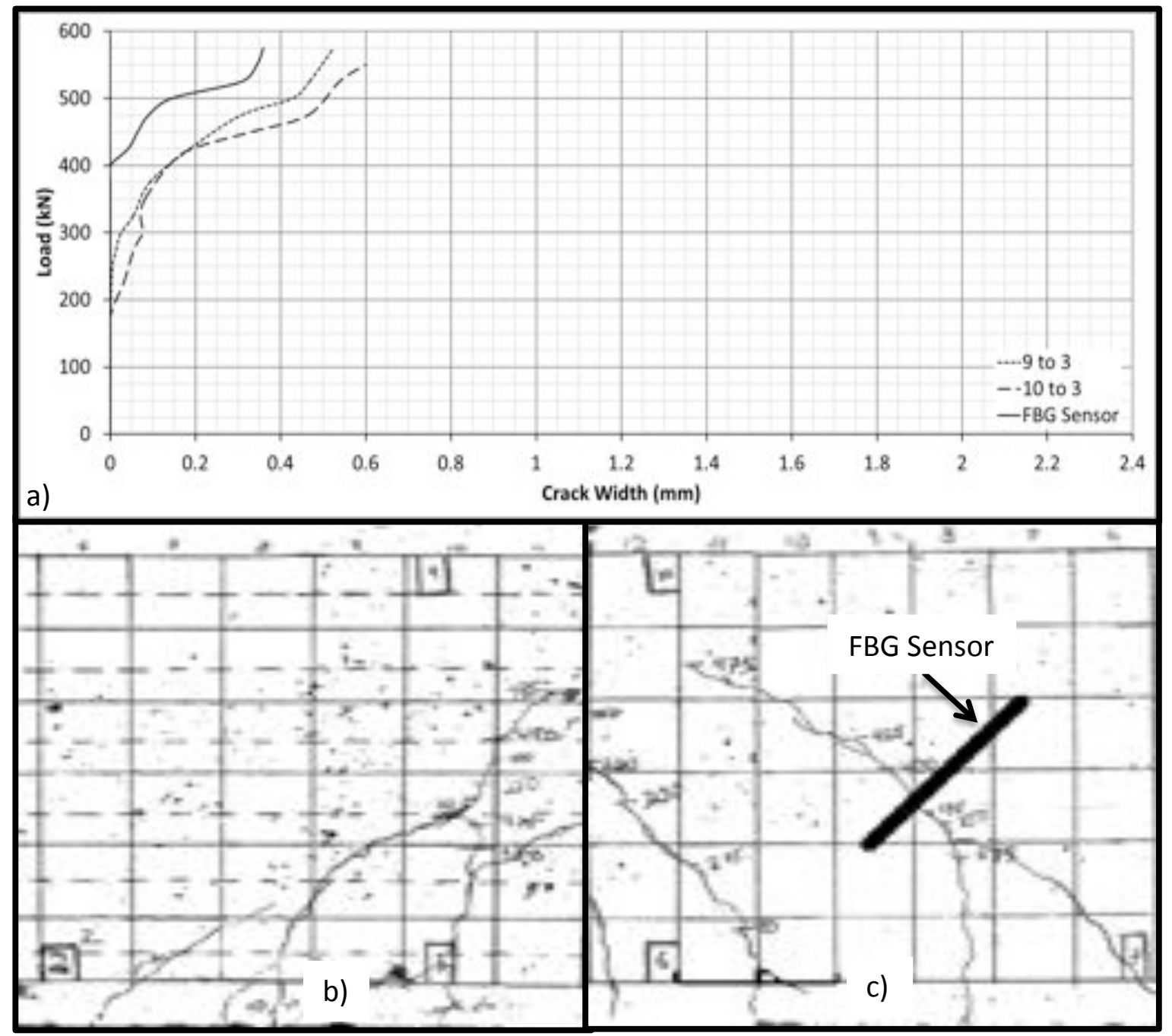

Figure 6.36: Shear Crack width measurements for beam HS-L-M-2; (a) Load-Crack Width Graph yielded by Piezo-Sensors and FBG Cable; (b) Observe cracking pattern between sensors Pair 9-3, left side of the beam; (c) Observed Cracking Pattern Between Sensor Pair 10-6, right side of the beam

The results of the fiber optic sensor do provide a means for interpreting the results obtained from sensor pair 10-6. As the crack that the fiber optic cable monitored, was seen to propagate through the effective gauge length of the fiber optic sensor at the $400 \mathrm{kN}$ load step. The crack measured by the piezo-sensors intersected the signal transmission at a load 
of $300 \mathrm{kN}$. The two devices are both measuring what is observed to be a shear crack, thus the behavior of the crack's prorogation is similar at any location within the beam. Knowing that the piezo-sensor crack developed $100 \mathrm{kN}$ prior to the one from the fiber optic cable the results would be expected to be similar in shape, and lagged by $100 \mathrm{kN}$. As the crack between the piezo-sensors initiated at a much earlier load step, than the crack measured by the fiber optic cable, the crack between the piezo-sensors is expected to develop a much larger crack width. Both of these results are confirmed from the Load-Crack Width graph in Figure 6.36 .

The left side of the beam showed very similar results. The propagation of the shear crack on this side was visually observed to intersect the signal path of sensor pair 9-3 at a load of 325 $\mathrm{kN}$, and detected by the sensor pair at $250 \mathrm{kN}$. The sensor pair monitoring the shear crack on the left half of member HS-L-M-2 also illustrates a similar trend for the Load-Crack Width graph presented in Figure 6.36a, demonstrating a similar behavior for the crack propagation. As on the right side of the beam, the outer most flexural shear crack on the left side of beam HS-L-M-2 is monitored by two sensor pairs. Sensor pair 2-3 and 9-3 effectively monitor the same crack at varying locations along its development. The initiation of the shear crack was captured at: $275 \mathrm{kN}$ for sensor pair 9-3, and $250 \mathrm{kN}$ for sensor 2-3. This result, as on the right side of the beam, suggest that the crack initiated as a shear crack at half of the beam's height, after which, as the load was increased, the shear crack intersected a flexural crack.

The crack width analysis conducted for beam HS-L-M-2 was intended to provide a means of determining the time instant when the member exceeded its serviceability limit state. The calculations provided by the code were carried out for the member, and yielded a serviceability limit of $326.7 \mathrm{kN}$. The results of the piezo-sensors consistently point to two sensor pairs, for the distinction of this specific load step. Sensor pairs 9-3 and 2-3, monitoring the left half of beam HS-L-M-2, both differentiate the $325 \mathrm{kN}$ load step, as both sensors demonstrate the initiation of cracking in the member very close to the $325 \mathrm{kN}$ load step. The shear crack of member HS-L-M-2 was expected to initiate at a load of $300 \mathrm{kN}$, as calculated. Thus the initiation of the shear crack in this member is very indicative of the 
member reaching its serviceability limit state, in this particular case. Although the approach for determining the limit state is indirect, it provides a good means of establishing a warning for maintenance engineers to assess the damage induced into the reinforced concrete member, and provides the required rehabilitation techniques as to not exceed the member's serviceability limit state.

\subsubsection{Results Obtained With the MIRA 3D Tomographer}

As conducted for Beam HS-L-M-1, the MIRA 3D tomographer was used to determine the location of reinforcing bars in beam HS-L-M-2, and map the cracking pattern of the member. For this, scans were taken only in the horizontal scan direction, as the results from beam HS-L-M-1 demonstrated that very little information about the beam's behaviors can be learned from the vertical scan. The results of beam HS-L-M-1 demonstrated the MIRA's ability to both accurately map the location of stirrups in the reinforced concrete beam, and the location of cracking. The result of the horizontal scans conducted on beam HS-L-M-2 is presented here in for both the healthy and damaged states of the member.

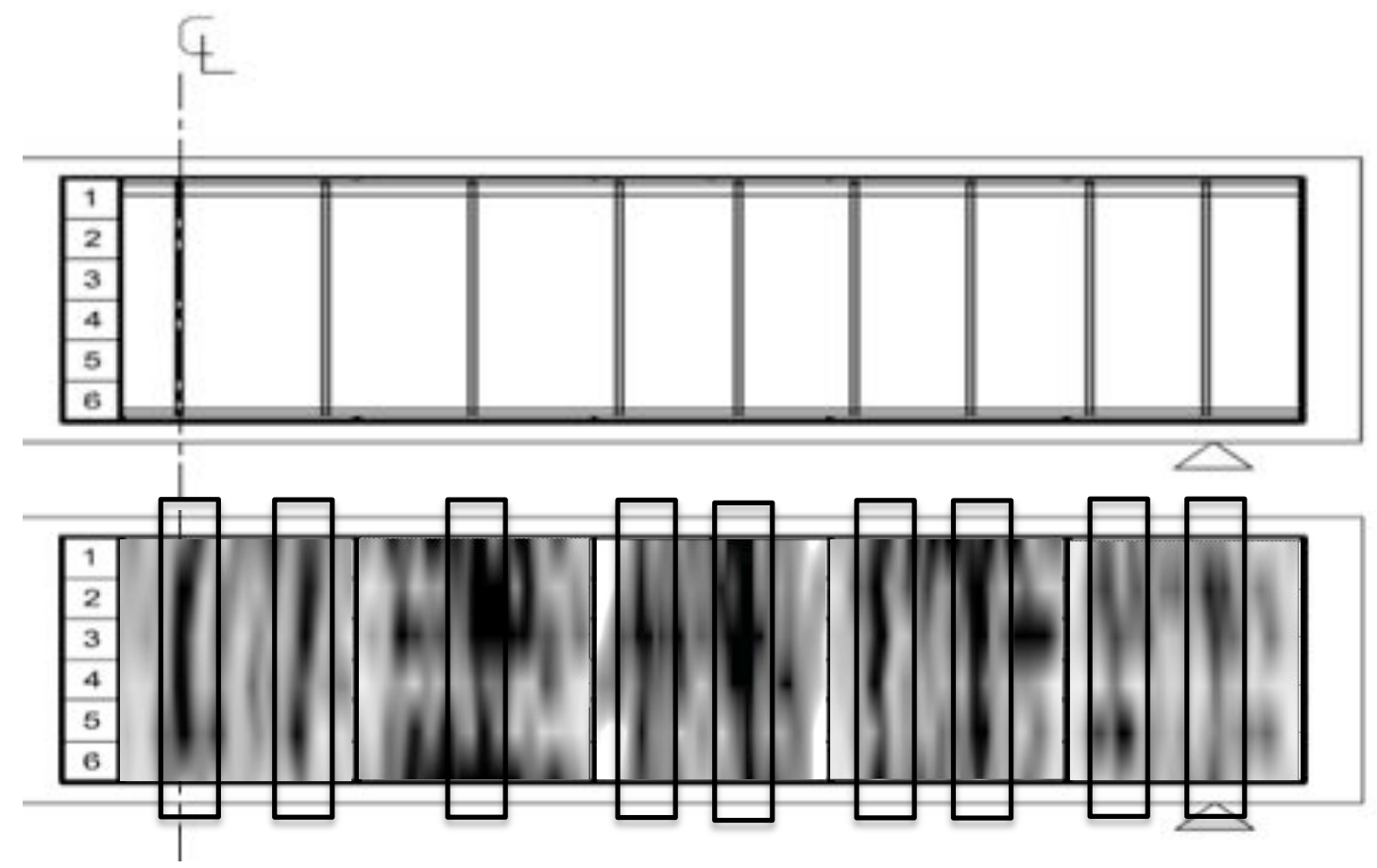

Figure 6.37: Horizontal Scan Direction Beam HS-L-M-2 Healthy State; (a) Theoretical Location of Steel Reinforcement; (b) MIRA Software Output 
Figure 6.37 illustrates the location of the steel reinforcing stirrups found in beam HS-L-M-2 (Figure 6.37a) as well as the software output obtained from the MIRA software upon scanning the beam's surface in the beam's healthy state (Figure 6.37b). The results obtained from the MIRA tomographer help establish a reference image that can be later used to map the damage induced while the beam was loaded. The result illustrated in Figure $6.37 \mathrm{~b}$ also provides a clear image of the internal location of the steel reinforcing bars highlighted with the nine black rectangles in the image. Just as in the analysis of beam HS-L-M-1, locating the reinforcing bars on the reference image will help differentiate between reflections obtained from the reinforcement bars, and those obtained from the damaged state of the beam.

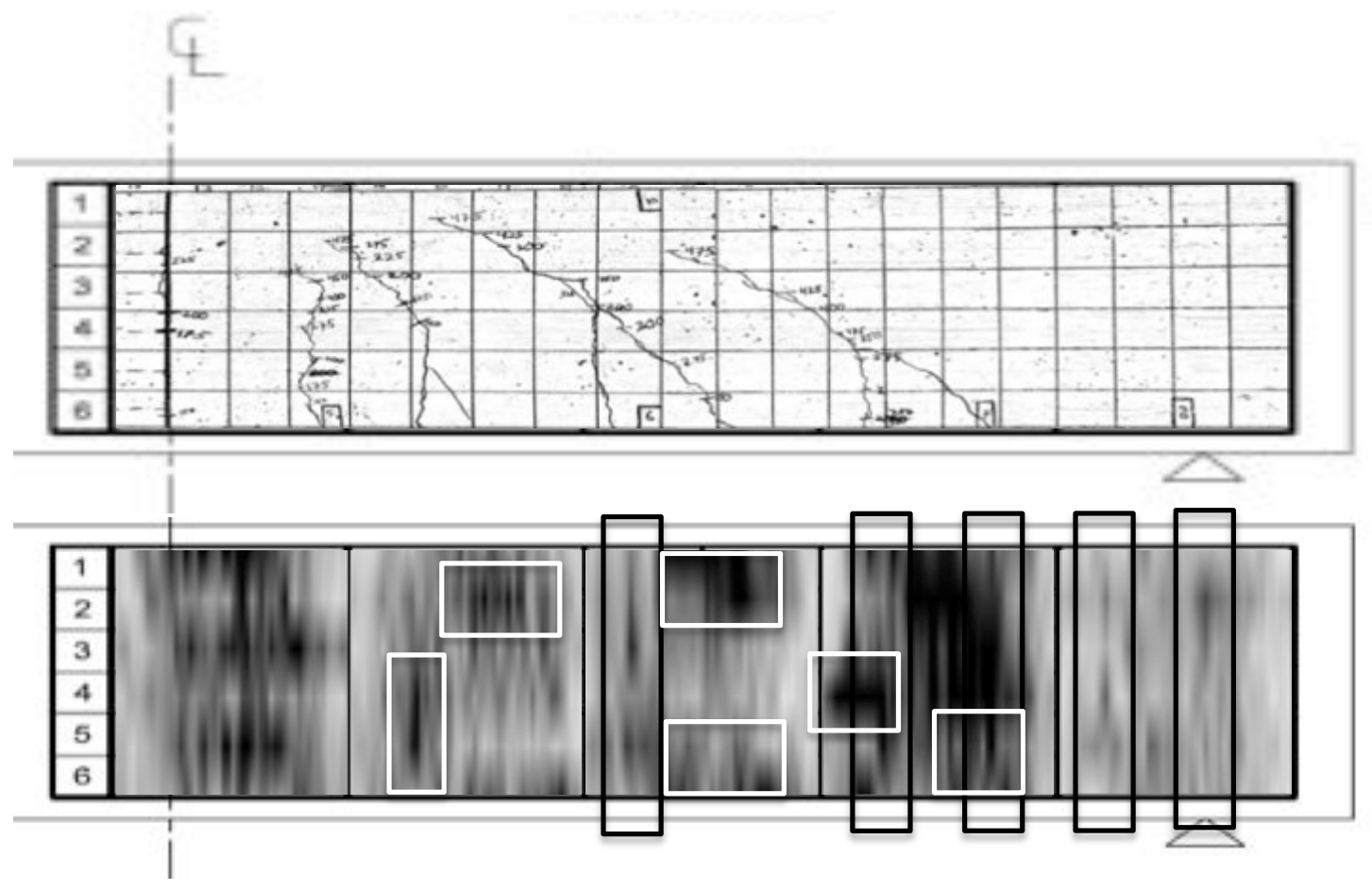

Figure 6.38: Horizontal Scan Direction Beam HS-L-M-2 Damaged State; (a) Observed Cracking Pattern on Scanned Surface; (b) MIRA Software Output

Figure 6.38 illustrates the observed cracking pattern once the beam exceeded its ultimate capacity (Figure 6.38a), and the software output of the MIRA tomographer (Figure 6.38b). As the results obtained from beam HS-L-M-1, the results obtained from the MIRA tomographer do demonstrate the appearance of cracking in the member. Although, the signal reflected from the reinforcement bars is not completely attenuated in this case. The 
first six reinforcement bars do still provide some reflection, and can still be differentiated on the tomograph in Figure 6.38b (black rectangles). The appearance of cracking in beam HS-LM-2 was concentrated to the mid-section of the beam, as expected of a flexural beam. The same mid-section where the concentration of cracking occurred, also presented a high amount of signal attenuation, completely blocking out the reflections obtained from the steel reinforcement present in this region. The MIRA tomographer also provided some information of the appearance of the shear crack. These are in form of higher reflections obtained from the regions surrounding the cracks, and are highlighted with the white rectangles in the image. The information provided by the MIRA tomograph is useful for locating damage, although the technique does not provide a means of measuring the widths of the cracks detected. 



\section{Chapter 7 $\quad$ Conclusion}

The proposed system proved to be a very effective tool for active condition assessment of reinforced concrete structures. The quick responsiveness and high signal output of the embedded piezo-sensors, along with their durability and ease of integration within the concrete matrix made them an ideal component of the nondestructive test system.

Throughout the preparation of the test specimens, the sensors proved to be very durable. The high heat produced during the curing process of the large-scale specimens had no effect on the working condition of these piezo-sensors. The casting process did not cause any signs of damage to the sensors. The piezoceramic patches used in the fabrication of the piezo-sensors have a very high durability to high temperature exposure, and the rugged epoxy encasing, makes them ideal for structures where mass concrete is used. Although the sensors were designed for heavy civil structures such as offshore and nuclear containment 
facilities, they are not limited to this sort of structure. The senses could be easily implemented into any reinforced concrete structure. Theoretically, the operating principle of the sensors would allow for their embedment into existing structures, although the investigation was not verified in this experimental work.

\subsection{Findings}

As proposed in the development of the system, the piezo-sensors were very effective in determining the initiation of cracking within a concrete member. The exceptional sensitivity of the system, not only detected the initiation of cracking within a member it also proved to be a useful tool for predictive health monitoring. By adopting the proposed $\mathrm{HMI}$ threshold of 0.5 , the system provides useful information as to when a member is expected to crack, and provides an early warning of distress. The implementation of this active monitoring system for real time continuous recording would help facilitate the maintenance of high importance, high risk facilities by providing engineers with accurate, up to date information about the building's condition. Using the information provided by these sensors, maintenance personal could prioritize, and effectively assess these crucial structures.

The developed HMI-Crack Width model for the system proved to be a very useful tool in the evaluation of the reinforced concrete beams. The piezo-sensor system demonstrated very reliable results, when compared to well establish system. The system also proved to be more reliable in the evaluation of members providing a complete consensus of the members' behaviors through their lifetimes. The piezoceramics endured the casting process, and continued to provide results up to the failure load of the member. The embedded piezo-sensors also demonstrated the means of establishing a beams overall condition. The various limit states established by the national building code of Canada were differentiable from the signal outputs solely making the system a very useful active nondestructive testing tool.

The signal outputs of the piezo-system were successfully processed to determine the development of concrete's early strength. The vibration characteristic of a section was 
successfully related to the development of strength of a sample, and the relationship illustrated by Equation 5.1 summarizes this result. By attaining the strength of a concrete sample 24 hours after casting, the piezo-system could project the strength gain for the following six days with very accurate precision. This result is very useful, as it would allow for the use of the same array of sensors that provide health monitoring information about a sample, during the construction phases of the building to prioritize the quality control of the cast concrete. The system can then provide a clear representation of the concrete's strength development on a member-to-member basis, rather than the global method relied on today.

The signal output of the piezo-sensors was also related the internal concrete strain experienced by a concrete section. The relationship was achieved through the investigation of small-scale experimental beams equipped with strain measuring equipment. The output signal was related to the measurements obtained by the strain measuring equipment to establish Equation 5.4. This equation provides a means of relating the output signal of the piezo-sensors (HMI) to the crack width intersecting the signal path. The relationship is summarized in Figure 6.15

The relationship obtained between the piezo-sensor signal, and the crack width was verified using a couple of full-scale experiment beams, that were equipped with Fiber Bragg Grating fiber optic sensors. A comparison between the two systems was drawn, as to verify the validity for the piezo-system, and the crack width relationship determined in the current research. Based on the results obtained the proposed piezo-sensor system demonstrated results that were in good agreement with those obtained by the FBG sensors. The resits of the full-scale experimental program also demonstrated the piezo-system's location based capabilities. The strategic distribution of piezo-sensors inside the concrete matrix illustrated one of the key advantages of using the piezo-system, over alternative systems. The proposed system was able to effectively assess the condition of an entire member based on the assessment of signals transferred between two adjacent sensors. By strategically distributing the sensor array along the beams' span, multiple sensor combinations can be used to effectively monitor the structural condition of a member on the entirety of its span. 
Unlike alternative embedded non-destructive systems, the proposed piezo-ceramic system is not limited to a specific gauge length, yet can monitor large sections of a reinforced concrete member with a single reading.

\subsection{MIRA 3D Tomographer}

The MIRA tomograph proves to be an effective tool for the non-destructive evaluation various structural components that is available on the market today. The equipment is successful in determining the placement condition of reinforcement in a concrete sample, and determining the punching shear failure of concrete slabs. Although the machine has a specified capability of reading signals to a depth of $2500 \mathrm{~mm}$, it may not be the case for concrete structures. Material inconsistencies such as gradation and reinforcement found the capability the MIRA tomograph to only about $1600 \mathrm{~mm}$ after which the signal is completely attenuated and there is very minimal reflections returned to the antenna leading to inaccurate and irrelevant reading of the internal structure. The equipment was also found to be successful for locating signs of distress, or cracking, in a concrete beam, although it is not successful in measuring the crack widths with ease.

\subsection{Recommendations for Future Research}

In the current investigation, the piezo-sensors have been proven to work in the assessment of flexural reinforced concrete members. The sensor array's was proven to provide accurate information about the initiation, location, and magnitude of the induced damage up to a maximum distance, sensors-to-sensors, of $700 \mathrm{~mm}$. The sensors also demonstrated the ability of transmitting a signal up to a maximum distance of $1600 \mathrm{~mm}$, although having no reference for the readings; the measurements could not be validated. In further investigations, the sensor-to-sensor distance needs to be increased, and the effectiveness

of crack width quantification needs to be verified. A full investigation needs to be carried out as to determine the optimum sensors-to-sensor distances, and the accuracy of the piezo-sensors system. 
Appendices 



\section{Appendix A \\ Compressive Strength Test Results Small Scale Beams}

Table A-1: Results Obtained for the Compressive Strength based on Standard Concrete Cylinders in MPa

\begin{tabular}{c|c|cccccccc}
\hline Cylinder & Day & $\mathbf{1}$ & $\mathbf{2}$ & $\mathbf{3}$ & $\mathbf{4}$ & $\mathbf{5}$ & $\mathbf{6}$ & $\mathbf{7}$ & $\mathbf{2 8}$ \\
\hline 1 & & - & 30.834 & 33.743 & 35.569 & 37.011 & 37.639 & 41.648 & 50.187 \\
2 & 22.746 & 30.778 & - & 34.494 & 36.384 & 38.873 & - & 54.669 \\
3 & 21.243 & 30.814 & 34.964 & 36.368 & - & 37.887 & 41.723 & 47.050 \\
\hline $\begin{array}{c}\text { Average } \\
\text { Compressiv } \\
\text { e Strength }\end{array}$ & 21.99 & 30.81 & 34.35 & 35.48 & 36.70 & 38.13 & 41.69 & 50.63 \\
\hline
\end{tabular}





\section{Appendix B | \\ Calculations for HS-L-M Beams}

Table B-1: Summary of Limit State Results

\begin{tabular}{c|cc}
\hline Summary Of Limit States & Moment (kN·m) & Applied Load (kN) \\
\hline Cracking & 121.3 & 167.3 \\
Serviceability Limit State & 236.9 & 326.7 \\
Factored Member Resistance & 355.3 & 490.0 \\
Ultimate Limit State & 412.6 & 569.1 \\
\hline
\end{tabular}

Cross-Section:

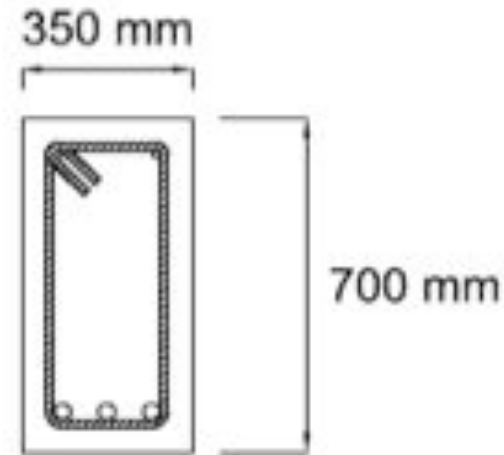

Concrete Properties:

$\alpha_{1}=0.85-0.0015 f^{\prime} \mathrm{c}=0.775$

$\beta_{1}=0.97-0.0215 f^{\prime} \mathrm{c}=0.775$

$E_{c}=4500 \sqrt{\mathrm{f}^{\prime} \mathrm{c}}=31,819.8 \mathrm{MPa}$

$f_{r}=0.6 \sqrt{\mathrm{f}^{\prime} \mathrm{c}}=4.24$

\begin{tabular}{c|c}
\hline Clear Cover (C.C.) & $50 \mathrm{~mm}$ \\
$\mathrm{I}_{\mathrm{g}}$ & $10.0 \times 10^{9}$ \\
$\mathrm{I}_{\mathrm{cr}}$ & $2.8 \times 10^{9}$ \\
$\mathrm{f}^{\prime}{ }_{\mathrm{c}}$ & $50 \mathrm{MPa}$ \\
$\mathrm{f}_{\mathrm{y}}$ & $450 \mathrm{MPa}$ \\
$\Phi_{\mathrm{s}}$ & 0.85 \\
$\Phi_{\mathrm{c}}$ & 0.65 \\
$\mathrm{~d}$ & $637.4 \mathrm{~mm}$ \\
Dia.bt $10 \mathrm{M}$ & $11.3 \mathrm{~mm}$ \\
Dia.bb $25 \mathrm{M}$ & $25.2 \mathrm{~mm}$ \\
\hline
\end{tabular}




\section{Resistant moment}

$$
\begin{gathered}
a=\frac{\emptyset_{s}\left(A_{s b}-A_{s t}\right) f_{y}}{\alpha_{1} \emptyset_{c} f^{\prime}{ }^{b} b}=\frac{(0.85)(3(500)-2(100))(450)}{(0.775)(0.65)(50)(350)}=56.4 \mathrm{~mm} \\
\begin{aligned}
M_{r}=\emptyset_{s}\left(A_{s b}-A_{s t}\right) f_{y}\left(d-\frac{a}{2}\right)+\emptyset_{s} A_{s t} f_{y}\left(d-c c-\frac{d i a_{\cdot b 1}}{2}\right) \\
=(0.85)[3(500)-2(100)](450)\left(637.4-\frac{56.4}{2}\right)+(0.85)[2(100)](450)(637.4-50 \\
\left.\quad-\frac{11.3}{2}\right)
\end{aligned}
\end{gathered}
$$

$M_{r}=355.28 \mathrm{kN} \cdot \mathrm{m}$

\section{Cracking Moment}

$M_{c r}=\frac{f_{r} I_{g}}{y_{t}}=\frac{(4.24)\left(10 \times 10^{9}\right)}{700 / 2}$

$M_{c r}=121.27 \mathrm{kN} \cdot \mathrm{m}$

\section{Serviceability Limit State}

$$
\begin{aligned}
& \frac{2}{3} f_{y}=\frac{2}{3}(450)=300 \mathrm{MPa} \\
& M_{r}=\emptyset_{s}\left(A_{s b}-A_{s t}\right) f_{(2 / 3) y}\left(d-\frac{a}{2}\right)+\emptyset_{s} A_{s t} f_{(2 / 3) y}\left(d-c c-\frac{d i a \cdot b 1}{2}\right) \\
& =(0.85)[3(500)-2(100)](300)\left(637.4-\frac{56.4}{2}\right)+(0.85)[2(100)](300)(637.4-50 \\
& \left.-\frac{11.3}{2}\right) \\
& M_{r}=236.9 \mathrm{kN} \cdot \mathrm{m}
\end{aligned}
$$




\section{Ultimate moment}

$$
\begin{gathered}
a=\frac{\left(A_{s b}-A_{s t}\right) f_{y}}{\alpha_{1} f_{c}^{\prime}{ }_{c}^{b}}=\frac{(3(500)-2(100))(450)}{(0.775)(50)(350)}=43.1 \mathrm{~mm} \\
M_{r}=\left(A_{s b}-A_{s t}\right) f_{y}\left(d-\frac{a}{2}\right)+A_{s t} f_{y}\left(d-c c-\frac{d i a \cdot b 1}{2}\right) \\
=[3(500)-2(100)](450)\left(637.4-\frac{43.1}{2}\right)+[2(100)](450)(637.4-50 \\
\left.-\frac{11.3}{2}\right)
\end{gathered}
$$

$M_{r}=412.6 \mathrm{kN} \cdot \mathrm{m}$ 



\section{Appendix C I \\ Crack Spacing and Crack Width Calculations}

\section{Average Crack Spacing at 575 kN:}

For $\mathrm{P}=575 \mathrm{kN} ; K_{1}=1$

$$
\begin{aligned}
& h_{\text {eff }}=\text { c.c. }+\frac{\text { dia }_{b b}}{2}+7.5\left(\text { dia }_{\cdot b b}\right)=50+\frac{25.2}{2}+7.5(25.2)=251.6 \mathrm{~mm} \\
& M=575 / 2 * 1.45 \mathrm{~m}=416.875 \mathrm{kN} \cdot \mathrm{m} \\
& f_{s}=\frac{M_{f}}{\emptyset_{s}\left(A_{s b}-A_{s t}\right)(d-a / 2)+\emptyset_{s} A_{s t}\left(d-\text { c.c. }-\frac{d i a_{b t}}{2}\right)} \\
& =\frac{416.875}{(0.9)(1500-200)(637.4-56.4 / 2)+(0.9)(200)\left(637.4-50-\frac{11}{2}\right)} \\
& =509.94 \mathrm{MPa}
\end{aligned}
$$

$$
\xi_{b}=\frac{f_{s}}{E_{s}}=\frac{509.94}{200,000}=0.00255
$$$$
\xi_{1}=\frac{\xi_{b}(h-a)}{d-a}=\frac{0.00255(700-56.4)}{637.4-56.4}=0.002824
$$$$
\xi_{2}=\frac{\xi_{1}\left(h-h_{e f f}-a\right)}{d-a}=\frac{0.00282(700-251.6-56.4)}{637.4-56.4}=0.001905
$$$$
k_{2}=\frac{0.25\left(\xi_{1}+\xi_{2}\right)}{2 \xi_{1}}=0.209 s_{m}=2(\text { c.c. }+0.1 s)+k_{1} k_{2} d_{b c} h_{e f f} \frac{b}{A_{s t}}
$$

$$
\begin{aligned}
& =2\left(50+0.1\left(\frac{350-100-20-}{2}\right.\right. \\
& +(1)(0.209)(25.2)(251.6) \frac{350}{1500}
\end{aligned}
$$

$S_{m}=244.36 \mathrm{~mm}$ 


\section{Average Crack Width}

$\mathrm{M}_{\mathrm{cr}}=121.27 \mathrm{kN} \cdot \mathrm{m}$

$$
\begin{aligned}
f_{s}=\frac{M_{c r}}{\emptyset_{s}\left(A_{s b}-\right.} & \left.A_{s t}\right)(d-a / 2)+\emptyset_{s} A_{s t}\left(d-c . c .-\frac{d i a_{b t}}{2}\right) \\
= & \frac{121.27}{(0.9)(1500-200)(637.4-56.4 / 2)+(0.9)(200)\left(637.4-50-\frac{11}{2}\right)} \\
= & 148.34 \mathrm{MPa}
\end{aligned}
$$

$$
\begin{aligned}
& r=1-\frac{\beta}{2.5 k_{1}}\left(\frac{\sigma_{s r}}{\sigma_{s}}\right)^{2} \\
& r=1-\frac{1}{2.5(0.4)}\left(\frac{148.34}{509.94}\right)^{2} \\
& r=0.92
\end{aligned}
$$

$$
\begin{aligned}
& w_{m}=r \varepsilon_{1} S_{m} \\
& w_{m}=(0.92)\left(\frac{509.94}{200,000}\right)(244.36) \\
& \boldsymbol{w}_{\boldsymbol{m}}=\mathbf{0 . 5 7} \mathbf{m m}
\end{aligned}
$$

$$
\begin{aligned}
& w_{k}=1.7 w_{m} \\
& w_{k}=1.7(0.57)
\end{aligned}
$$

$w_{k}=0.97 \mathrm{~mm}$ 
Table C-1: Summary of Average and Maximum Crack Width Calculations by Section of Beam HS-L-M-2

\begin{tabular}{|c|c|c|c|c|c|c|c|c|c|c|}
\hline \multirow{2}{*}{$\begin{array}{c}\text { Applied } \\
\text { Load } \\
\text { (kN) }\end{array}$} & \multicolumn{2}{|c|}{$\begin{array}{c}2-3 \\
650-1050\end{array}$} & \multicolumn{2}{|c|}{$\begin{array}{c}3-4 \\
1050-1750\end{array}$} & \multicolumn{2}{|c|}{$\begin{array}{c}4-5 \\
1750-2250\end{array}$} & \multicolumn{2}{|c|}{$\begin{array}{c}5-6 \\
2250-2750\end{array}$} & \multicolumn{2}{|c|}{$\begin{array}{c}6-7 \\
2750-3350\end{array}$} \\
\hline & $W_{m}$ & $w_{k}$ & $w_{m}$ & $w_{k}$ & $W_{m}$ & $w_{k}$ & $W_{m}$ & $w_{k}$ & $W_{m}$ & $w_{k}$ \\
\hline 0 & & & & & & & & & & \\
\hline 25 & & & & & & & & & & \\
\hline 50 & & & & & & & & & & \\
\hline 75 & & & & & & & & & & \\
\hline 100 & & & & & & & & & & \\
\hline 125 & & & & & & & & & & \\
\hline 150 & & & & & & & & & & \\
\hline 175 & & & & & 0.02 & 0.03 & & & & \\
\hline 200 & & & & & 0.07 & 0.11 & 0.01 & 0.02 & & \\
\hline 225 & & & 0.02 & 0.04 & 0.11 & 0.19 & 0.05 & 0.09 & & \\
\hline 250 & & & 0.06 & 0.10 & 0.15 & 0.25 & 0.09 & 0.16 & & \\
\hline 275 & & & 0.10 & 0.16 & 0.19 & 0.32 & 0.13 & 0.22 & & \\
\hline 300 & & & 0.13 & 0.22 & 0.22 & 0.38 & 0.16 & 0.28 & & \\
\hline 325 & & & 0.16 & 0.27 & 0.26 & 0.44 & 0.19 & 0.33 & & \\
\hline 350 & & & 0.19 & 0.32 & 0.29 & 0.50 & 0.23 & 0.38 & 0.00 & 0.01 \\
\hline 375 & & & 0.22 & 0.37 & 0.33 & 0.55 & 0.26 & 0.43 & 0.03 & 0.05 \\
\hline 400 & & & 0.25 & 0.42 & 0.36 & 0.61 & 0.28 & 0.48 & 0.05 & 0.09 \\
\hline 425 & 0.02 & 0.03 & 0.27 & 0.47 & 0.39 & 0.66 & 0.31 & 0.53 & 0.07 & 0.13 \\
\hline 450 & 0.04 & 0.07 & 0.30 & 0.51 & 0.42 & 0.71 & 0.34 & 0.58 & 0.10 & 0.16 \\
\hline 475 & 0.06 & 0.10 & 0.33 & 0.56 & 0.45 & 0.77 & 0.37 & 0.63 & 0.12 & 0.20 \\
\hline 500 & 0.08 & 0.13 & 0.35 & 0.60 & 0.48 & 0.82 & 0.40 & 0.67 & 0.14 & 0.23 \\
\hline 525 & 0.10 & 0.16 & 0.38 & 0.64 & 0.51 & 0.87 & 0.42 & 0.72 & 0.16 & 0.26 \\
\hline 550 & 0.11 & 0.19 & 0.40 & 0.68 & 0.54 & 0.92 & 0.45 & 0.76 & 0.17 & 0.29 \\
\hline 575 & 0.13 & 0.22 & 0.43 & 0.73 & 0.57 & 0.97 & 0.47 & 0.81 & 0.19 & 0.33 \\
\hline
\end{tabular}





\section{Reference List}

Achenbach, D. P. (1973). Wave Propagation in Elastic Solids. Amsterdam: Elsevier.

Aggelis, D. G., \& Shiotani, T. (2007). Repair evaluation of concrete cracks using surface and through-transmission wave measurements. Cement \& Concrete Composites , 29 (9), 700711.

Annamdas, V. G., Yang, Y., \& Soh, C. K. (2010). Impedance based Concrete Monitoring using Embedded PZT Sensors. International journal of Civil and Structural Engineering , 1 (1), 414424.

Ayotte, E., Massicotte, B., Houde, J., \& Gocevski, V. (1997). Modeling the thermal stresses at early ages in a concrete monolith . ACI Mater. J. , 577-587.

CSA. CAN/CSA-A23.3-04 (R2010) - Design of Concrete Structures. Missisauga, Ontario, Canada: Canadian Standard Associations.

CSA. (2004). S474 Concrete Structures. Missisauga, Ontario, Canada: Canadian Standard Associations.

Dawood, N. F., \& Marzouk, H. (2010). Behaiour of Steel Reinforced Concrete Panels Subjected to Direct Tension. Memorial University of Newfoundland, Faculty of Engineering and Applied Science. St. John's: Memorial University of Newfoundland.

De La Haza, A. O., Petersen, C. G., Samokrutov, A., De La Haza, A. O., Petersen, C. G., \& Samokrutov, A. Three Dimensional Imaging of Concrete Structures Using Ultrasonic Shear Wave. Germann Instruments. Germann Instruments.

Duan, Z., Ou , J., Zhou, Z., \& Zhao, X. (2005). Smart Sensors and Integrated SHM Systems for Structural Health Monitoring. In Springer, \& F. Ansari (Ed.), Sensing Issues in Civil Structural Health Monitoring (pp. 269-278). Dordrecht, Netherlands: Springer.

Gaydecki, P. A., Burdenkin, F. M., Demaj, W., John, D. G., \& Payne, P. A. (1991). The propagation and attenuation of medium-frequency ultrasonic waves in concrete: a signal analytical approach . Meas. Sci. Technol. (3), 126-134. 
Germann Instruments, I. (2010). MIRA 3D Tomographer User Manual. Germann Instruments, Inc.

Gudra, T., \& Stawiski, B. (2000). Non-destructive strength characterization of concrete using surface waves. Wroclaw: Elsevier.

Guo, S., Zhang, Z., \& Yang, S. (2011, March 10). Longitudinal waves in one dimensional nonuniform waveguides. Theoretical \& Applied Mechanics Letters .

Kovalev, A., Rasmussen, J., \& Shaw, P. (2002). Ultrasonic testing of concrete structures. 8th European Conference on Nondestructive Testing . 8. Barcelona: Spanish Society for NDT (AEND).

Li, Z., Qin, L., \& Huang, S. (2009). Embedded Piezo-Transducer in Concrete for Property Diagnosis . Journal of Materials in Civil Engineering , 21 (11).

Lin, Y. B., Chang, K. C., Chem, J. C., \& Wang, L. A. (2004). The health monitoring of a prestressed concrete beam by using fiber Bragg grating sensors. Smart Materials and Structures, 13 (4), 712-718.

Malhotra, V. M. (1984). In situ/nondestructive testing of concrete. Michigan, United States of America: American Concrete Institute.

Rao, M. V., \& Lakshmi, K. (2003). Shear-wave propagation in rocks and other lossy media: An experimental study . Current Science, 85 (8), 1221-1225.

Rizk, E., Marzouk, H., \& Hussein, A. (2011). Punching Shear of Thick Plates with and without Shear Reinforcement. ACl Structural Journal , 108 (5), 581-591.

Rizk, E., Marzouk, H., Hussein, A., \& Gu, X. (2012). Structural health monitoting of slabcolumn connections using FBG sensors. Journal of Civil Stuctural Health Monitoring , 2 (1), 17-27.

Sansalone, M., \& Streett, W. B. (2007). Impact-echo: non-destructive evaluation of concrete and masonry. Michigan, United States of America: Bullbrier Press. 
Song, G., Gu, H., \& Mo, Y.-L. (2008). Smart aggregates: multi-functional sensors for concrete structures-a tutorial and a review. Smart Materials and Structures, 17 (3), 1-17.

Song, G., Gu, H., Hsu, T. T., \& Dhonde, H. (2007). Concrete structural health monitoring using embedded piezoceramic transducers. Smart Materials and Structures , 16 (4), 959968.

Standardization, N. C. (1992). NS34773E Concrete Structures Design Rules. Norway: Norwegian Council for Building Standardization.

Sun, M., Staszewski, W. J., \& Swamy, R. N. (2010). Smart Sensing Technologies for Structural Health Monitoring of Civil Engineering Structures. (J. Zhu, Ed.) Advances in Civil Engineering , 2010, 1-13.

Sun, W., \& Yan, S. (2008). Health monitoring strategy for smart piezoelectric concrete structures. In M. Tomizuka (Ed.), SPIE. 6932. SPIE.

Voigt, T., Sun, Z., \& Shah, S. P. (2006). Comparison of ultrasonic wave reflection method and maturity method in evaluating early-age compressive strength of mortar. Cement \& Concrete Composites , 28 (4), 307-316.

Wang, C. H., \& Weng, Y. K. (2000). Evaluation of pre-Northridge low-rise steel buildings. I: modeling (Vol. 126). Journal of Structural Engineering.

Wang, C. S., Wu, F., \& Chang, F.-K. (2001). Structural health monitoring from fiberreinforced composites to steel-reinforced concrete. Smart Materials and Structures , 10 (3), 548-552.

Wu, F., \& Chang, F.-K. (2006). Debond Detection using Embedded Piezoelectric Elements for Reinforced Concrete Structures - Part II: Analysis and Algorithm . Structural Health Monitoring , 1 (5), 17-28.

Wu, F., \& Chang, F.-K. (2006). Debond Detection using Embedded Piezoelectric Elements in Reinforced Concrete Structures - Part I: Experiment . Structural Health Monitoring , 1 (5), 515. 
Wu, T. T., Fang, J. S., Liu , G. Y., \& Kuo , M. K. (1995). Determination of Elastic Constants of a Concrete Specimen Using Transient Elastic Waves. J. Acoust. Soc. Am. , 4 (98), 2142-2148.

Zhao, X., Li, H., \& Jinglong, W. (2008). Concrete Structure Monitoring Based on Built-in Piezoelectric ceramic Transducers. In M. Tomizuka (Ed.), SPIE. 6932. SPIE. 